\title{
Algorithmic Toolmaking:
}

Parameter Thinking in Landscape Architecture

Kadin Hegglun

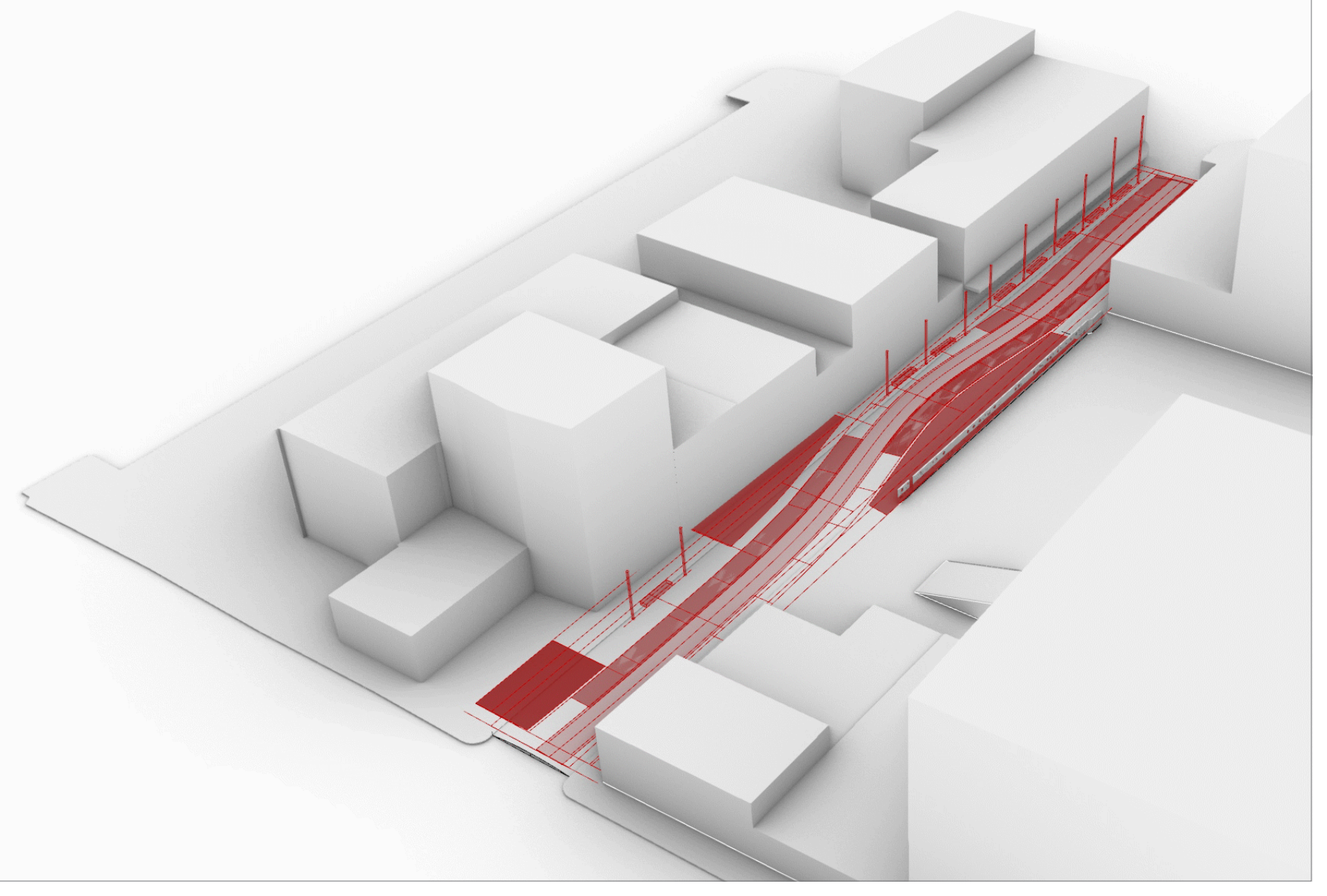




\title{
Algorithmic Toolmaking: Parameter Thinking in Landscape Architecture
}

By

\author{
Kadin Hegglun
}

A thesis submitted to the Victoria University of Wellington in fulfilment of the requirements for the degree of Landscape Architect

Victoria University of Wellington

2020

Page II | Preface 


\section{Acknowledgments}

This thesis is dedicated my parents, Jocelyn and John Hegglun.

Thank you for being so supportive of my education.

Thank you to my primary supervisor Brennan Baxley - for the consistent guidance and weekly morning catchups at Mojo Cafe.

Thank you to my secondary supervisor Hannah Hopewell - for words of wisdom throughout the process. 


\begin{abstract}
This thesis interrogates the contentious integration of digital technologies into the field of landscape architecture. Identifying that an application of computational technique is largely unknown, the research delves into the scripting of geometry relationships with the use of the tool 'Grasshopper' in the context of landscape architecture.

The discourse surrounding the appropriation of digital technology in assistance to landscape architecture is seemingly controversial with an apparent resistance in favour of traditional methodologies of site design.

It is commonly stated that, digital software tends to be 'too architectural' and therefore less tailored to the open systems that landscape architects contend with. The tendency of contemporary software is to mimic the analogue process and while useful in representation and drawing production - these programs are scaleless and detached from reality. It is affirmed by academics such as Bradley Cantrell and Caroline Westort that landscape architecture needs more algorithmic attention. Stressing the construction of relationships between design-move and site condition.

Parameter thinking infers a method of rule setting and dedication to the settling of boundaries in which the potentials of site intervention has room to shift. Geometries, points, curves and planes provide such palette.

Abstraction is a primary conceptual driver of any drawing convention yet Grasshopper offers a more dynamic and animated process of conceptual development. The abstraction of site into such geometries grants a dynamic, shifting and generative paradigm to design and toolmaking.

This thesis is to unfold the paradigm of operating within such a toolset adopting the use of Grasshopper almost exclusively as a way of engaging with conceptual development. Such an interface allows this thesis to note the performance of an algorithmic toolset and adopt an algorithmic mind set.
\end{abstract}




\section{Contents}

$\begin{array}{lc}\text { Glossary } & \text { VII } \\ \text { Introduction } & 1 \\ \ldots \ldots \ldots \ldots \ldots \ldots \ldots \ldots \ldots \ldots \ldots \ldots \ldots \ldots \ldots \ldots \ldots \ldots \ldots \ldots \ldots \ldots \ldots \ldots \ldots \ldots \ldots \ldots \ldots \ldots \ldots \ldots \ldots \ldots \ldots \ldots \ldots \\ \text { Discourse Trend } & 2 \\ \text { Thesis Methodology } & 4\end{array}$

Review Structure

Literature review $\quad 6$

Thesis Positioning / Precedent Study 14

$\begin{array}{ll}\text { A Palette of Points, Curves and Planes } & 18\end{array}$

$\begin{array}{ll}\text { Disclaimers } & 19\end{array}$

Study $1 \quad 20$

$\begin{array}{ll}\text { Approaching Site } & 22\end{array}$

The Principal $\quad 24$

Logics $\quad 24$

Region Logics $\quad 30$

Parking Logics $\quad 32$

Street Side Logics 33

$\begin{array}{ll}\text { A Conceptual Machine } & 41\end{array}$

Script Interface $\quad 42$

Making Variations $\quad 44$

Study 1 Conclusion $\quad 54$

Study 2

Microclimates 58

$\begin{array}{ll}\text { Pattern and Simulation } & 65\end{array}$ 
$\begin{array}{ll}\text { Points and Density } & 68\end{array}$

Mesh Manipulation $\quad 69$

$\begin{array}{ll}\text { Toolmaking } & 71\end{array}$

$\begin{array}{ll}\text { Path Evaluation } & 73\end{array}$

$\begin{array}{ll}\text { Feedback Loops } & 74\end{array}$

$\begin{array}{ll}\text { Variations } & 79\end{array}$

Study 2 Conclusion $\quad 82$

Critical Reflection $\quad 84$

$\begin{array}{lr}\text { Study } 3 & 88\end{array}$

Porirua Stream Potential Engagement 89

Paradigm 96

Generative Logics 108

Interface 118

Script Management 126

Tool 132

$\begin{array}{ll}\text { Conclusion } & 138\end{array}$

Thesis Conclusion 140 


\section{Glossary}

Some terms are conceived in this thesis to help build a vocabulary around the appropriation of algorithmic thinking in landscape architecture, regarding also the technicalities of programming/scripting. Other terms were picked from Grasshopper and literature. Reflect to this section for descriptions of terms used.

- $\quad$ Algorithmic Thinking: Describes the ability for designers to think from a perspective of computer language, in this thesis regarding landscape architecture.

- $\quad$ Geometric Relationship Structuring: Refers to the process of scripting in regard to points, curves and planes. Emphasising the construction of relationships between geometries as opposed to the geometries themselves.

- Logic: A loosely used term to describe a cluster of components(Grasshopper syntax) that form a design action. Generally it is used to refer to primary functions of the script.

- Multifaceted Thinking: Describes the ability for designers to think through multiple design trajectories simultaneously under an algorithmic paradigm.

- Parameter: A boundary, limitation or extent in which guides the action of a logic. A vital property of the script as it defines what a design element can and cant do. Logics impose parameters on other logics, giving hierarchy to the script.

- $\quad$ Parameter Thinking: The ability for designers to think in terms of the extents that a design element can potentially for exist in, as opposed an assured position. Necessary for dynamic modelling. 
- $\quad$ Region: An enclosed area, typically defined by a curve or surface. A region has clear boundaries in which Grasshopper is able to recognise the area within.

- $\quad$ Principal: The first element in a script structure. This term evolves over the course of the thesis, it comes to regard closely the design intention of the designer due to the tendency for a principal to be a dominant feature of the script. 
\begin{tabular}{l|l} 
Page $\mathbf{x}$ & Page intentionally left blank
\end{tabular} 


\section{Introduction}

Digital technologies have had a slow integration into landscape architecture. Although it is recognised that current software that is available to landscape architects such as AutoCAD is not all that suitable for conceiving design ideas. Instead only appropriated for means of representation and detail drawing production, reproducing what has already been designed by analogue methods. It seems in landscape architecture, we are clutching at software designed for architects, and while parametric software has taken the architectural world by storm, we try to incorporate the same software into landscape architecture to contend with a different palette, undefined lines, temporality, weather condition, this often does not work so well. Landscape architects work in complex environments and so our tools need to be adaptive and sensitive to the propinquity of site.

This thesis recognises the folly of contemporary software, yet refuses to suggest that digital technology cannot be adapted to the conceptual process of landscape architectural design. This is inspired by algorithmic thinking, which has the potential to invoke the linking of ideas with the condition of site in a dynamic three dimensional model. Discussion surrounding the use of algorithmic tools in a landscape architectural context has arose over the past decade, some academics believing programing should be taught to landscape architects in the face of the technological golden age.

To explore this notion, this research adopts an algorithmic toolset through the appropriation of the tool, Grasshopper, which is embedded in the software Rhinoceros 6 . The thesis exhibits the multi-functionality of the visual scripting, delving into a novel way of thinking, describing a tedious yet versatile paradigm. The research concerns itself primarily with the conceptual design process of landscape architecture and is guided by the question...

\section{How can parametric software and algorithmic thinking support conceptual design within landscape architecture?}




\section{Discourse Trend}

Of the readings considered in this thesis, addressing the use of digital tools in landscape architecture, a visible shift in discourse can be seen from discussion of representation, modelling and digital drawing to discussions in programming, simulation and coding.
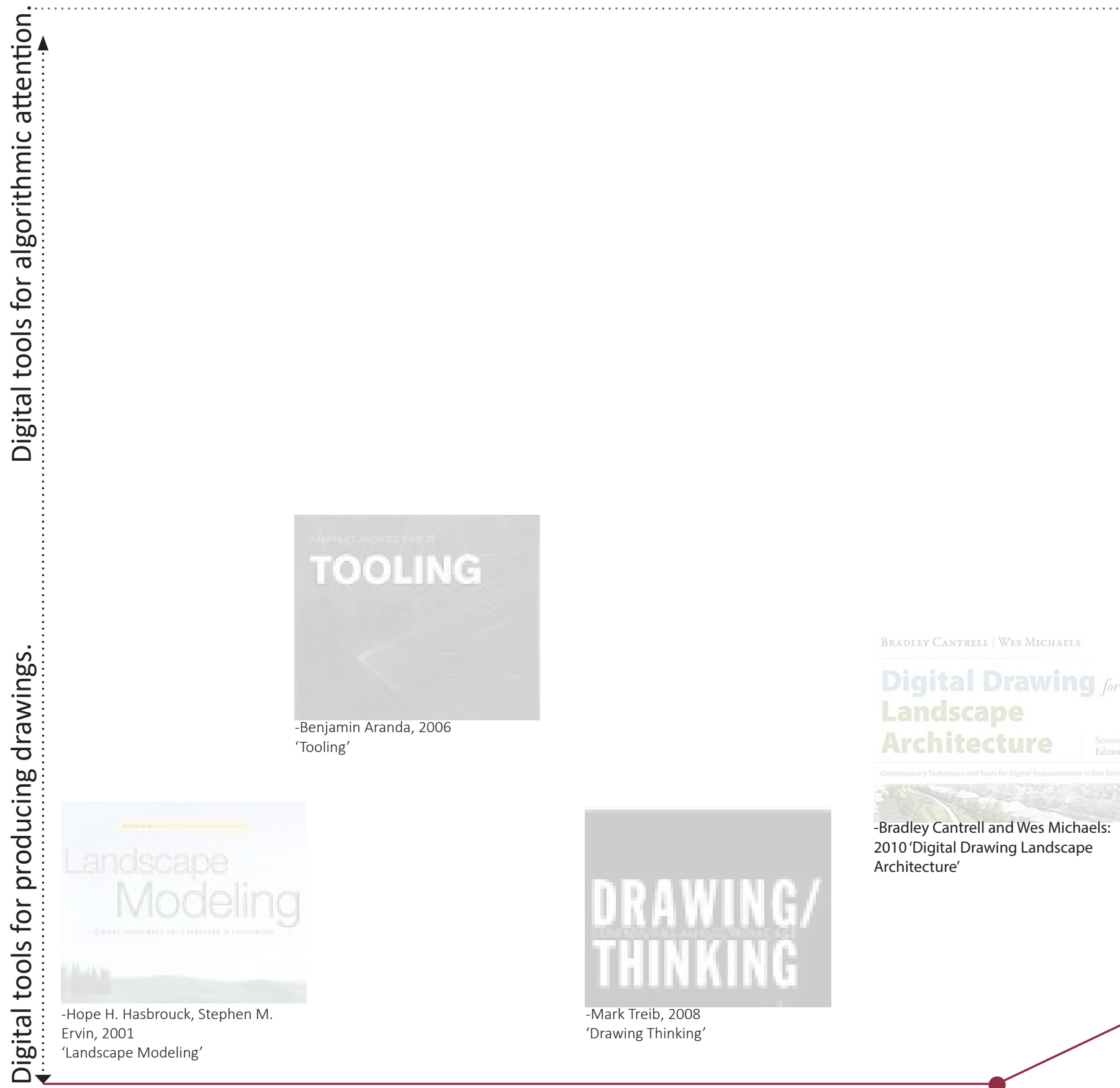

Bradley Cantrell and Wes Michaels: 2010`Digital Drawing Landscape Architecture' 


\section{Thesis Alignment}

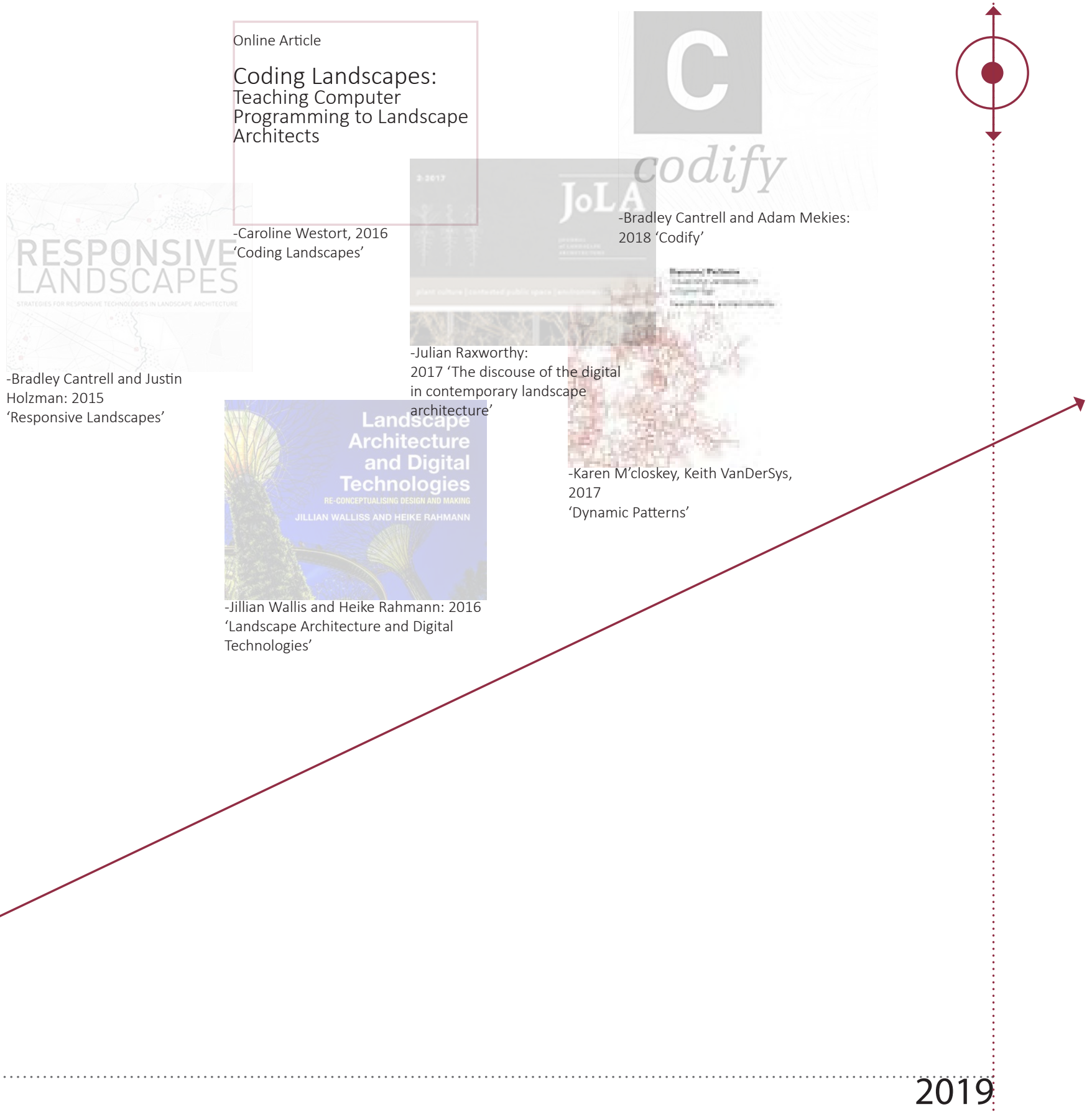

Discourse Trend $\quad$ Page 3 


\section{Thesis Methodology}

The trend following the discourse surrounding parametrics in Landscape Architecture suggests a movement from computation as a means for representation and drawing production towards discussions of algorithmic thinking, simulation and computation design. The research will be concerned primarily within the present dialogue. The methodology aims to facilitate means of extracting thought process and computational logic in relation to landscape architecture.

Thesis Aims and Objectives.

- Unfold new thought paradigm. How parametrics change the perception of site and design. Regarding coding thought, tool making and problem solving.

- $\quad$ Analyse and comment on the hindrances, requirements and potential of user-interface in relation to creative engagement.

- Within two studies; apply the use of the tool 'Grasshopper' under the model of variable relationship structuring.

- Apply discoveries and evolving theory in a final design scenario, commenting on design process and reflecting upon common models of site design. 


\section{Preparation}

Literature Review

Precedent Study

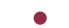

Research Positioning

\section{Study 1}

\section{Site Establishment}

Document the fundamentals of Grasshopper and algorithmic thinking pertaining to the site through conceptual design.

Study Reflection

\section{Study 2}

\section{Site Establishment \\ (Opposing conditions to first site)}

Document the change in process and algorithmic thinking pertaining to the site through conceptual design, building upon previously established ideas

Study Reflection

Critical Reflection, establish scope for study 3

\section{Study 3}

\section{Site Establishment}

Revisit concieved ideas, display the application of findings in a final design process.

Study Reflection

\section{Conclusion}




\section{Review Structure}

The following literature review is split into four headings. These headings, while an attempt to extend broadly into most facets of parametrics in landscape architecture, are key themes that will be discussed and reconsidered throughout this research.

Addressing the 'Resistance', the struggle of adopting parametric tools into landscape architectural practice, questioning why this struggle persists despite technological innovation.

Secondly, the theme 'Paradigm' investigates the mode of thinking involved with coding knowledge, how knowledge of coding tools may provide new perception of site and new approaches to design response.

Thirdly 'Tool' speaks generally to function and the application of digital tools.

Finally, 'Interface' addresses the designer-software relationship, noting creative potential and impairment wherein operating these tools.

\section{Resistance}

It is suggested by Bradley Cantrell and Adam Mekies that the struggle to integrate digital methods into landscape architecture is a struggle of translation between the tools of computation and the creativity of the designer and a struggle of syntax (computer language).

They note the adaption of analogue tools in computer software and hardware for the past two decades has imitated the traditional analogue methods with its use of pens, pencils and marks as is prevalent in the basic toolset of AutoCAD with its mouse-drawn lines and curves. (Cantrell \& Mekies, 2018)

"Rather than claiming that there is a "digital" media, we have instead adopted computation as a simulator and optimizer of analogue systems" (Cantrell \& Mekies, 2018)

Jillian Wallis and Heike Rahmann suggest many landscape architects have difficulties in conceptualising digital technology as a creative medium. They suggest this attitude can be traced to 3 issues. The conceptualisation of creativity as a human endeavour, the assumption that an unmediated connection between hand and the brain evident in the act of drawing provides the most valuable insight for design, and that technology distances the designer from the real world. Further they note this is reflected in academia. Citing Marc Treib's Drawing/Thinking: Confronting an Electronic Age (2008) and Representing landscape (2008) 
as works displaying anxiety over digital integration. Treib pushes for hesitancy and resistance to adopt computer processes, advocating hand drawing as having higher attention to reality, detail and complexity of life. He equates the limitations of CAD to having similar disconnects one perceives comparing sketch to photograph. (Treib, 2008)

"Where one works on a relatively small screen, line by line constructing drawings with no real scale, encouraging the study of parts removed from wholes." (Treib, 2008)

He affirms constraints are a characteristic of every and medium but stresses...

"The seeming immediacy and conquest of space and time through vehicles such as

cell-phones to some degree disguise a consequent removal from the complexity and propinquity of life"

(Treib, 2008)

Treib does not however denote the potential of new software and hardware developments to engage with the physical and the bodily. Instead distrusts Imitative and scale-less nature of CAD. (Treib, 2008)

\section{Paradigm}

Cantrell and Mekies's research seems to move away from drawing imitation software and instead focuses upon aspects of simulation and computation in which they fore-hope greater connection between computer and physical reality.

"Beyond the inherent responsibility of the designer comes the potential of an informed design process that can design within performance models, sketching with data itself, to test the potential of the site." (Cantrell \& Mekies, 2018)

Designing with data directly gives us the ability to foreshadow and to project, providing a cause and effect base to work within. The nature of input/output testing implies an efficiency, where inputs (a value) can be altered to specific requirements and an output response will result in a dynamic and immediate fashion.

Computer programming in its complex and rule abiding syntax requires an immensely different understanding of design process. It requires a unique interpretation of traditional design language and thinking. 
It stresses the linking of ideas, and interaction between parts of the problem and the solution. Computational thinking combines the powerful orderly process of algorithmic organization with the equally powerful, but more chaotic, process of iterative design." (Cantrell \& Mekies, 2018)

Assuming the nature of code, site analysis might pursue the extraction of physical variability and the formation of appropriate relationships between existing condition and design intention. Thereby scripting a set of actions in which formal outcome is produced. The line between site analysis and design concept is somewhat blurred by this model. The importance of the designer in the role of mediating these relationships is vital.

"In parametric modelling the importance of composition or geometries is replaced by the declaration of specific parameters or rules which become foundational to design outcome. This design approach shifts the focus from form as outcome to processes of formation, emphasising relationships over composition." (Wallis \& Rahman, 2016)

\section{Tool}

As we shift into a computer based society and as large detailed datasets evolve, it is vital we have means to play, translate and manipulate these data sets in meaningful and creative ways. The method of data translation I would argue is the biggest hurdle in becoming a digitally based practice.

Bradley Cantrell in Responsive Landscapes (2016) refers to the notion of feedback loops as being one such model of translation.

"This process of feedback, sensing the environment, processing the sensed data, and visualizing the response is the core design focus in the development of responsive technologies." (Cantrell \& Holzman, 2016)

Operating within a feedback loop calls for a response to any given action a designer might impose on the landscape. It suggests new paradigms for design process and emerging software development. Feedback models enable hybridisation of multiple datasets such as geology, climate change, flora and fauna etc.

The authors call for "parallel virtual models that run concurrently to create a hybrid feedback loop for the evaluation of multiple possible design trajectories in real time." (Cantrell \& Holzman, 2016) 
This type of model requires advancements in three things - Increased capabilities to process large amounts of data, mostly through developments in computational power. Secondly GUI (interface) must be enabling and engaging in order for designers to use such models in powerful and meaningful ways. Thirdly, advancements in sensory technology is required for the collection of large quantities of data. (Cantrell \& Holzman, 2016)

The later issue may be resolved through the evolution of Big Data. Understanding Big Data as a decentralized internet of large-volume, complex, growing data sets with multiple, autonomous sources including sensory. A digital model will intrinsically engage, manipulate, discover complex relationships and restructure datasets in pursuit of ideal local condition. (Wu, Zhu, Wu, \& Ding, 2014)

"The term Big Data reflects far more than quantity, instead describing data that can be considered to be 'generated continuously, seeking to be exhaustive and fine-grained in scope, and flexible and scalable in their production'." (Wallis \& Rahman, 2016)

Knowledge in coding allows designers to become toolmakers, having the ability to customise software to their own needs and the needs of the site. Coding, from a toolmaking perspective, offers a high level of flexibility and creativity while engaging with the inherent properties of computation- mathematical accuracy and efficiency. Freedom to manipulate and interpret datasets into form through means of scripted translation through tools such as Grasshopper and languages such as Python. (Wallis \& Rahman, 2016)

\section{Interface}

While advancements in technology will lead to greater accuracy of computer simulation, in order for landscape architects to engage with new programs in a meaningful and creatively empowering manner, we must bridge our bodily understandings of space and time with the software we use.

To achieve this, GUI (graphic user interface) of design software must be developed alongside the advancements of computational efficiency.

Grasshopper is revered for making huge progress in this barrier in its translation of the python scripting language into a visually interactive interface. This means that designers have access to a coding based toolset where they may have previously been discouraged by the tedious, boring nature of text based coding. (Cantrell \& Mekies, 2018) 


\section{Interface}

Caroline Westort of lowa State University asserts that it is important for landscape architects to know the fundamentals of computer programming for two primary reasons...

Current software that is available for landscape architects rarely fulfils the criteria for effective landscape design software. This aligns with Mark Treib's critique of CAD. She suggests secondly that digital representation of our landscape palette needs more algorithmic attention. (Westort, 2016)

"Curved surfaces, fuzzy edges, large scales characterize our palette, and remain limited, unwieldy and largely divorced from analytical functionality in design software used in design studio."

It may be that advancements in software GUI must be developed in order to engage landscape architects creatively and thoughtfully with digital tools, but perhaps it is also the responsibility of the future landscape architect to learn the fundamentals of computer coding. Otherwise, a specialist position in practice might be suggested, that being, the tool maker.

\section{Summary}

The digital landscape architectural discourse is a constantly developing discussion. As the technological revolution shifts forward and as we move into the era of Big Data, the discourse of this particular topic is left with a short window of opportunity to discuss. Leaving much literature surpassing five years obsolete in their criticality of contemporary technology.

Because of this time-frame, the most compelling literature in this review fits within the five year window. Namely 'Codify: Parametric and Computational Design in Landscape Architecture' by Bradley Cantrell and Adam Meikies (2018) and 'Landscape Architecture and Digital Technologies' by Jillian Wallis and Heike Rahmann (2016). While this challenges digital based academics it also expresses need for more discussion and a greater variety of perspective.

The trend of the discourse followed discussions linked to lan Mcharg's 'Design with Nature' (1969) and the production and spread of GIS throughout the 80's and 90's. The early 2000's saw resistance to GIS based practice considered to be constrained within "scientific positivist methodologies". (Wallis \& Rahman, 2016) 
Digital technologies has remained a slow and hesitant integration into landscape architecture with authors such as Marc Tribe advocating for traditional techniques in response to the looming "electronic age". (Treib, 2008) With design software being primarily an imitation of traditional methods since the 2000's - recent years have seen more literature seeking the computational application of coding based toolsets for design problem sovling.

While digital tools extend their use into a variety of different applications within landscape architecture- seemingly, the most potential for digital integration falls under the responsive applications of computation. Bradley Cantrell and Justine Holzman seek application of sensory technology in the pursuit of responsive modes of engagement based within digital models that operate in real-time.

Julian Raxworthy critiques this concept within his coverage of the digital discourse. He suggests the model must maintain a relationship with the world in real time, or close to it, in order for these simulations

to bring a level of defensibility to decision making in landscape architecture. Though he does glorify the model's potential if the case is so. (Raxworthy, 2017)

Limited is the current computational power we are currently operating with. As landscape architects deal with a continuum of multi-dimensional systems, current computation is unable to process the sheer amount of data required to be representational of lifelike complexity. Thus, Cantrell and Holzman's hybridized feedback loop remains theoretical.

One stitch in the veil that shrouds computational application is its drastically different approach to design thinking. What encourages this thesis deeply is how this paradigm shift effects the perception a designer might have of site design. To the nature of parametric modelling...

"The linking of ideas and interaction between parts of the problem and the solution." (Cantrell \& Mekies, 2018)

"Shifts the focus from form as outcome to processes of formation, emphasising relationships over composition" (Wallis \& Rahman, 2016)

This already suggests a different perception of site, where one looks for extents of potentiality rather than potentiality as singular. Therefore enabling a multi-faceted approach to design process.

To conclude, it is important not to underestimate the power of tool making that coding knowledge enables. In the hands of the designer it implies an interpretive and creative structure of problem solving yet calculated and precise. 


\section{References}

Cantrell, B., \& Holzman, J. (2016). Responsive Landscapes: Strategies for Responsive Technologies in Landscape Architecture. New York: Routledge.

Cantrell, B., \& Mekies, A. (2018). Codify: Parametric and Computational Design in Landscape Architecture. Routledge.

Raxworthy, J. (2017). The Discourse of the Digital in Contemporary Landscape Architecture. JoLA, 88-93.

Treib, M. (2008). Drawing/Thinking: Confronting an Electronic Age. New York: Taylor and Francis.

Wallis, J., \& Rahman, H. (2016). Landscape Architecture and Digital Technologies. New York: Routledge.

Westort, C. (2016). Coding Landscapes: Teaching Computer Coding to Landscape Architects. Iowa State University Digital Repository, pp. 337345.

Wu, X., Zhu, X., Wu, G.-Q., \& Ding, W. (2014). Data mining with Big Data. IEEE Transactions on Knowlede and Data Engineering, 97-107. 


\section{Thesis Positioning}

The appropriation of digital tools in landscape architecture is varied yet remains ambiguous regarding a clear use. The multi-application of Grasshopper for example has lead to many projects using the tool at different scales and in different scenarios. Hence the following collection of precedents using an array of digital applications, in comparison with one another they will ideally give a better understanding of both - Parametric Landscape Architecture and the research trajectory of this thesis.

Addressing the ambiguity of this tool, the types of parametric utility is best categorised in Jillian Wallis' and Heike Rahmann's 'Landscape Architecture and Digital Technologies', each chapter of the book investigates the different uses in contemporary parametric landscape architecture. Introducing first, the 'Topographic Surface' concerning surface morphology. Followed by 'Performative systems' engaging with the behaviour or performance of a system such as climate or stratosphere data. 'Simulating systems' digitally simulating otherwise invisible forces such as wind tunnels or hydrological data in order to test design potential with real time feedback. 'Materiality and Fabrication' Identifying potential in construction process and component fabrication. Finally they address 'Collaboration' in which the interdisciplinary communication potential is exemplified through tools such as BIM software. (Wallis \& Rahman, 2016)

This research concerns itself primarily with topographic surface, simulating systems and the generative qualities of algorithmic tools. Sternly, it looks into the relation of digital geometries and how a rounded understanding of parameter thinking can drive conceptual development. Therefore the precedents most relevant to the thesis have the clearest use of Rhinoceros geometry and relationship forming. Ordered from left to right in terms of intrigue and relevance. 


\section{Content unavailable}

Please consult print version for access.
Content unavailable

Please consult print version for access.
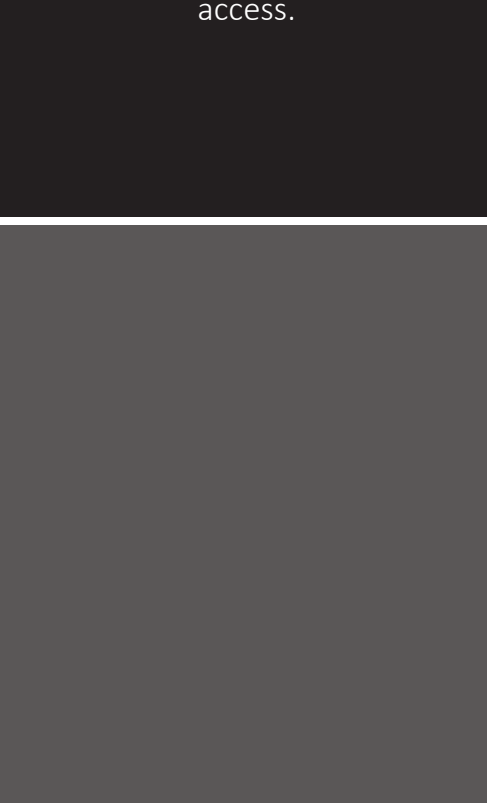

Figure 0.02. Phase Shifts Park (Wallis \& Rahman, 2016, wh. 48-61)

\section{Phase Shifts Park, Catherine Mosbach and Philippe Rahm. 2016}

Project type:

Public park

Application:

-Using software to engage with Taiwan's fluctuating climate. Mapping climatic shifts through computational fluid dynamics simulation. Engaging directly with climate and stratosphere data to inform design decision.

Keywords:

-Performative Systems

-Climate

-Sensory
Content unavailable

Please consult print version for access.

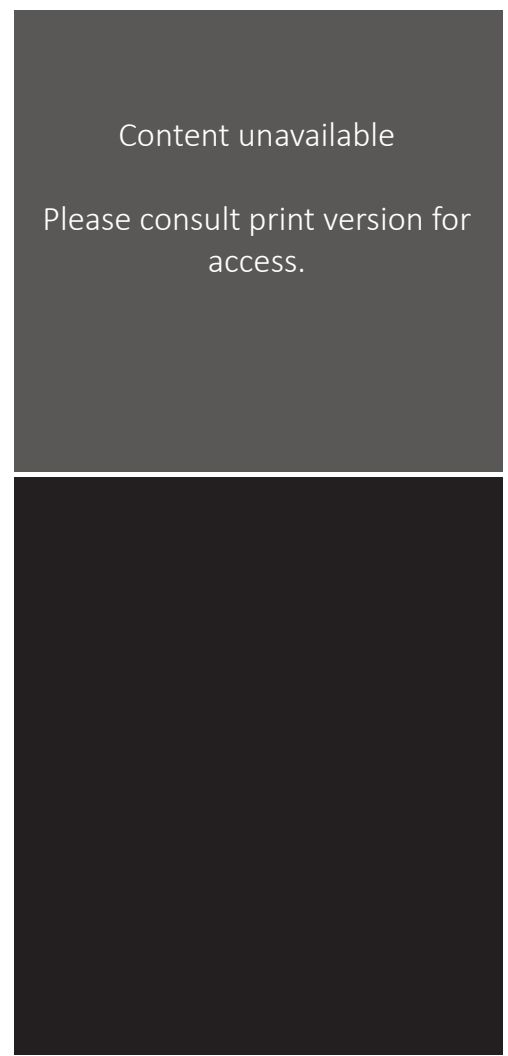

Figure 0.03. Landhausplatz (Wallis \& Rahman, 2016, wh. 16-19)

Landhausplatz, LAAC

Architekten and Stiefe

Kramar Architecture. 2011

\section{Project type:}

Large civic public square.

Application:

Using the software Rhinoceros and Maya. These programs aided in the production of smooth yet complex surfaces. Highlighting the precision and material qualities of parametrics where highly controlled cuts were made to the concrete. Simplifying construction and design of the surface.

\section{Keywords:}

-Materiality \& Fabrication

-Topographic Surface 


\section{Content unavailable}

Please consult print version for access.
Figure 0.03. MAX Lav IV (Wallis \& Rahman, 2016, wh. 34-41)

MAX Lab IV, Snøhetta. 2011

Project type:

Large scale complex.

\section{Application:}

-Using Grasshopperand Rhinoceros. Mounds were generated by creating spiral patterns enabling accurate calculation of cut and fill while controlling variations of size and intensity. Designers highlight the flexibility in the algorithm's ability to adapt to new information.

\section{Keywords:}

-Topographic Surface -Pattern
Content unavailable

Please consult print version for access.

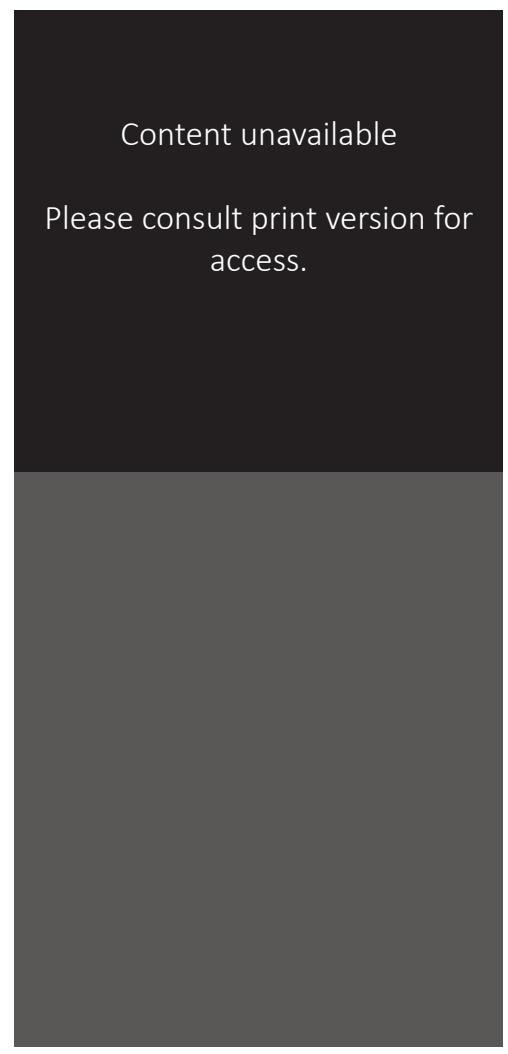

Figure 0.04. Bellwether (Wallis \& Rahman, 2016, wh. 72-76)

\section{Bellwether, PEG Office of Landscape + Architecture.}

2011

Project type:

Public park, competition entry (Same competition as Phase Shift Park)

Application:

-Using Grasshopper and Rhinoceros. Conceptually driven by procedural diagrammatic masterplan using attractor and repellent logics to tether variables together.

Keywords:

-Responsive

-Procedural

-Performative Systems
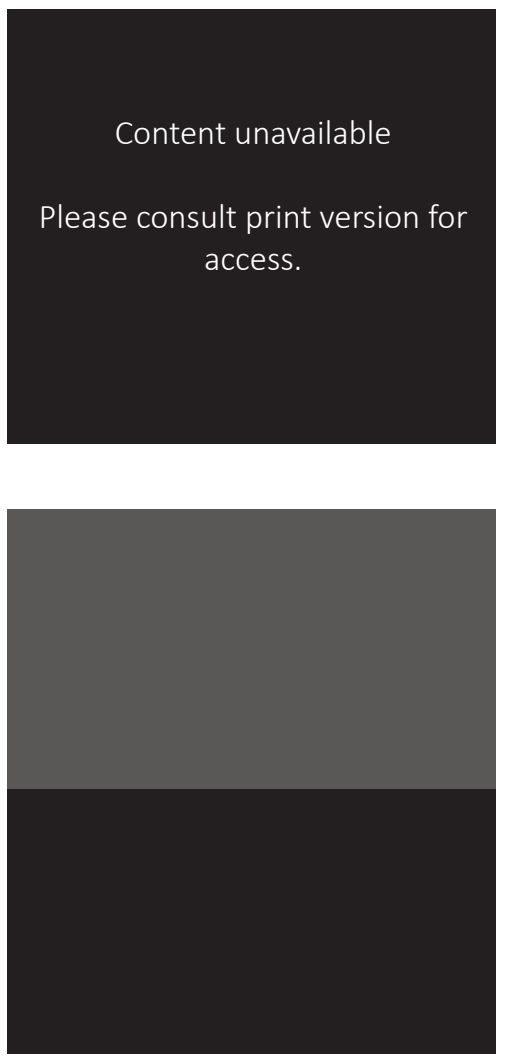

Figure 0.05. South Park, Sanfrancisco (Cantrell \& Mekies, 2018, p. 84-89)

\section{South Park, Fletcher Studio.}

2017

\section{Project type:}

Small public park

\section{Application:}

-Using Grasshopper and Rhinoceros. Applying weighting values to attractor and repellent points that were used to define the width and centerline of the central pathway. Points were linked to site entry, pedestrian desire lines and building type. Enabling careful consideration of circulation.

Keywords:

-Responsive

-Procedural

-Performative Systems 


\section{South Park, San Francisco}

Using Grasshopper, Fletcher Studio generated the pathway that runs the length of the linear park space. The path was manipulated through weighting factors that had been assigned to points. The geometry relationship here being; the curve (the path) and the point (relating to condition). The site conditions included site entry, desire lines and building type. The site boundaries provide parameter for the central curve to move between. Fig.0.08 illustrates this relationship.

The central path divides the linear park spatially. By prescribing pulling factors to the points the script then can generate an infinite amount of possibilities.

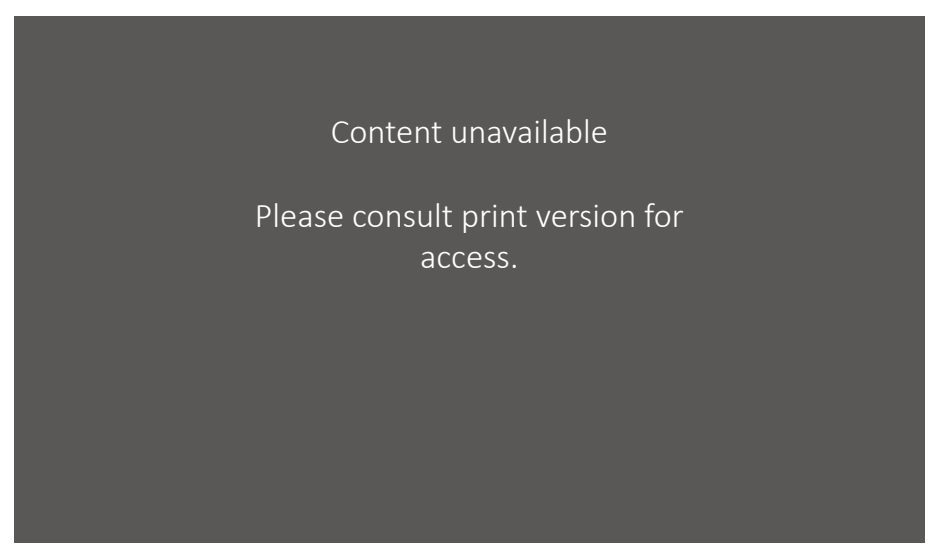

Figure 0.06. Aerial view of South Park, San Francisco, California (Cantrell \& Mekies, 2018, p. 84)

As the outcome is defined by the input values, the outputted design is known to some degree also. While this is true, the designer only knows the extents the script can operate in, as they defined them, and not the form that is created as a result of that. It is because of this partial "known" that makes this a powerful iteration generator, as it gives control to the randomness by reducing or expanding parameters. (Cantrell \& Mekies, 2018, pp. 84-89)

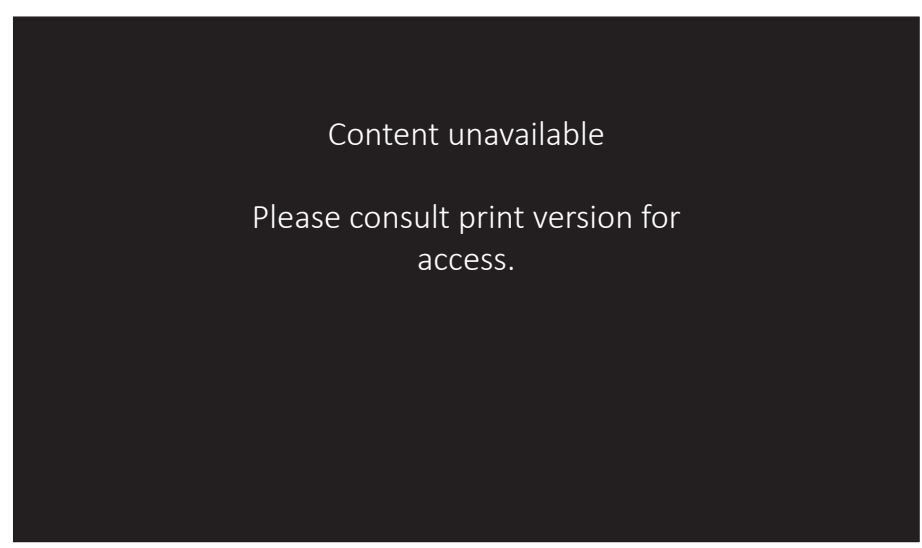

Figure 0.07. (above) Site feature prioritization and weighting diagram (Cantrell \& Mekies, 2018, p. 87). 


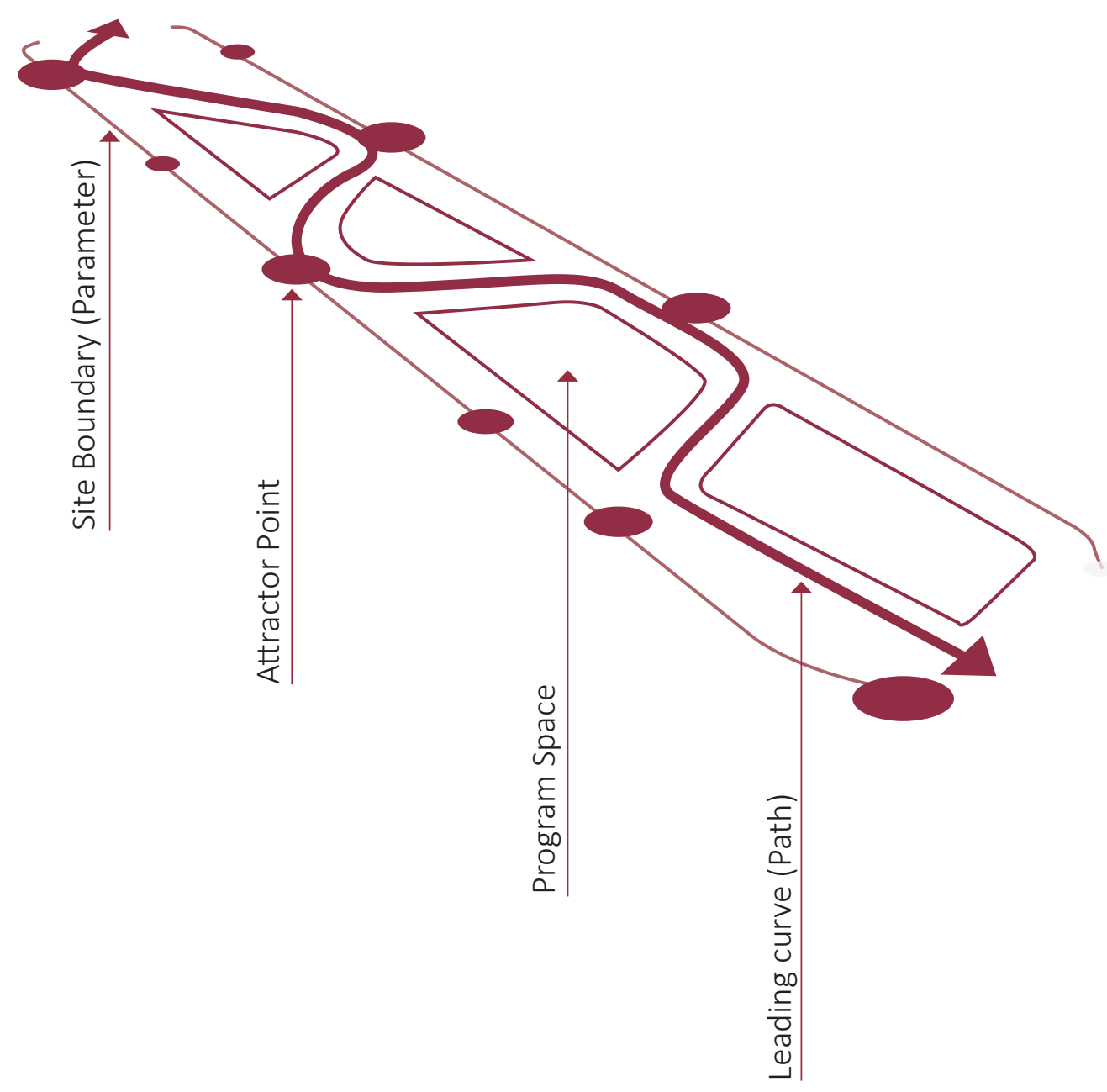

Figure 0.08. Authors abstraction of South Park, Sanfrancisco parameteric method. 


\section{A Palette of Points, Curves and Planes}

"Shapes are never unwilled figures. Deep within them is a struggle between the predilections of the architect and the inherent properties of the geometries encountered." (Aranda \& Lasch, 2006)

Points, lines, curves, planes, surfaces and meshes make up the parametric palette. It is the forming of relationships between these geometries that gives rise to formal outcome. Like sketching with pen on paper, a mark is made by firstly pressing the nib into the paper and stroking in a certain direction until you lift your hand to finish the mark. In code, similarly, an initial point $(A)$ is created in model space, followed by a second point (B). A subsequent logic for a line may then be generated between the two points, based on the xyz location of each. Naturally this imposes hierarchy - the line being formed by prior information. These steps may be repeated until a series of points and interconnecting lines have been created, from this information polygons might be created, then lofted to form solids. With this principle in mind complex geometric relationships may be formed. (Menges \& Ahlquist, 2011)

Algorithmic thinking is inherently rule based. The algorithm or script written by the programmer/designer simply put; tells the geometries to do something. A complex geometric design structure consists of a series of commands sequential and relative to each preceding piece of code. In a dymaic model, a rule, or better put, a parameter - is driven by pressure applied by another parameter that is itself contending with other parameters. Therefore it is the limitations that the parameters impose on one another that create form. This idea helps to visualise the concept of relationship structuring, implying importance more-so to the logic, rules and relationships between the geometries, rather than the geometries themselves. (Aranda \& Lasch, 2006)

All 2D and 3D geometric models are composed of some combination of points and lines. It is the kinds of additional properties such as inside or outside, left of, right of, closest to or farthest from, that can be calculated and utilised to provide parameters for subsequent geometries. A vector derived from one curve might prescribe the movement of the next. (Ervin \& Hasbrouck, 2001)

"Parametrics might be understood, conventionally, as regarding solely the interdependencies of certain geometrical constraints. But deeper understanding is that of the establishing methods for interrelating particular behaviours of forms and forces, and how they might be represented as associated mathematical and geometric rules." (Menges \& Ahlquist, 2011) 
It is 'Interrelating' as a keyword that is most exciting in regards to Landscape Architecture.

Advancements in computing, simulation and interface may one day lead to creatively engaging means of operating with and within open systems as it is described by Ludwig Von Bertalanffy with the notion of General Systems Theory. Whereas the system is characterized by the interactions of its components and the nonlinearity of those interactions, implying a hierarchy, cross relational and scaling relationship amongst systems. (Walonick, 1993)

Similarly points and curves acting as a complex relative and dynamic structure, cross relate and scale much the same under the pressures of hierarchical rule setting. While it is in the interest of this thesis scope to focus on the structuring of these geometric relationships, it is vital to consider the significance of their complexity and the potential for a more sensitive and responsive way of designing in a planet that at the largest scale, operates as one system.

\section{Disclaimers}

\section{It's new to me}

-The flow of this research will follow my own development in the learning for Grasshopper. The merit of this fact will enable for clear declaration of the learning curve taken to learn the software. This will also effect the pace of which complexity in the use of the tool evolves.

\section{Vocabulary}

-As there tends to be a lot of words vernacular to the parametric discourse, a glossary is supplied on page 4 . Words best descriptive of a given concept will be introduced If existing descriptions are not present.

\section{The CPU Struggle}

-As CPU (Computer Processing Power) is considered resource where the average computer can only handle so much data being processed at once, effecting the true viability of some of the scripts. This thesis to some degree considers this factor obsolete and instead assuming CPU will not be such an issue in the near future with the advancements of computer technology. 


\section{Study 1 Introduction}

Similar to South Park, San Francisco (discussed on pg 16) - Tory Street, Wellington is linear and confined in its composition, it was ideal that this site provided simple dimensions for establishing a basic understanding of algorithmic process regarding Rhinoceros and Grasshopper geometries. This factor enabled a testing environment concerned primarily with the 2 dimensional properties of the street surface.

The study brought necessity for hierarchy amongst a sequential and branching script structure in which adjustments to a logic (actions relating to the model, see glossary) would affect local actions and the logics succeeding it, not effecting preceding logics, any logic higher in the hierarchy.

This gave basic definition to algorithmic thinking under the notion of the Principal, the logic and the parameter.

This structure enabled a deeper understanding of Rhinoceros geometries, particularly points, curves and surfaces. Manipulated under a string of logics these geometry's ability to relate to one another is crucial to the fundamentals of Rhino and Grasshopper. The thought and principles behind them however transcending the software.

Conclusively, the designed tool enabled quick iteration and manipulation of the streets basic properties.

\section{Study One, Aims and Objectives}

- Understand and establish basic relationship structuring in the context of an urban street.

- $\quad$ Extract thought pattern/process pertaining to algorithmic rules and restrictions.

- $\quad$ Establish a vocabulary regarding key ideas.

- Understand the basic properties of Rhinoceros geometries, points, curves etc. 


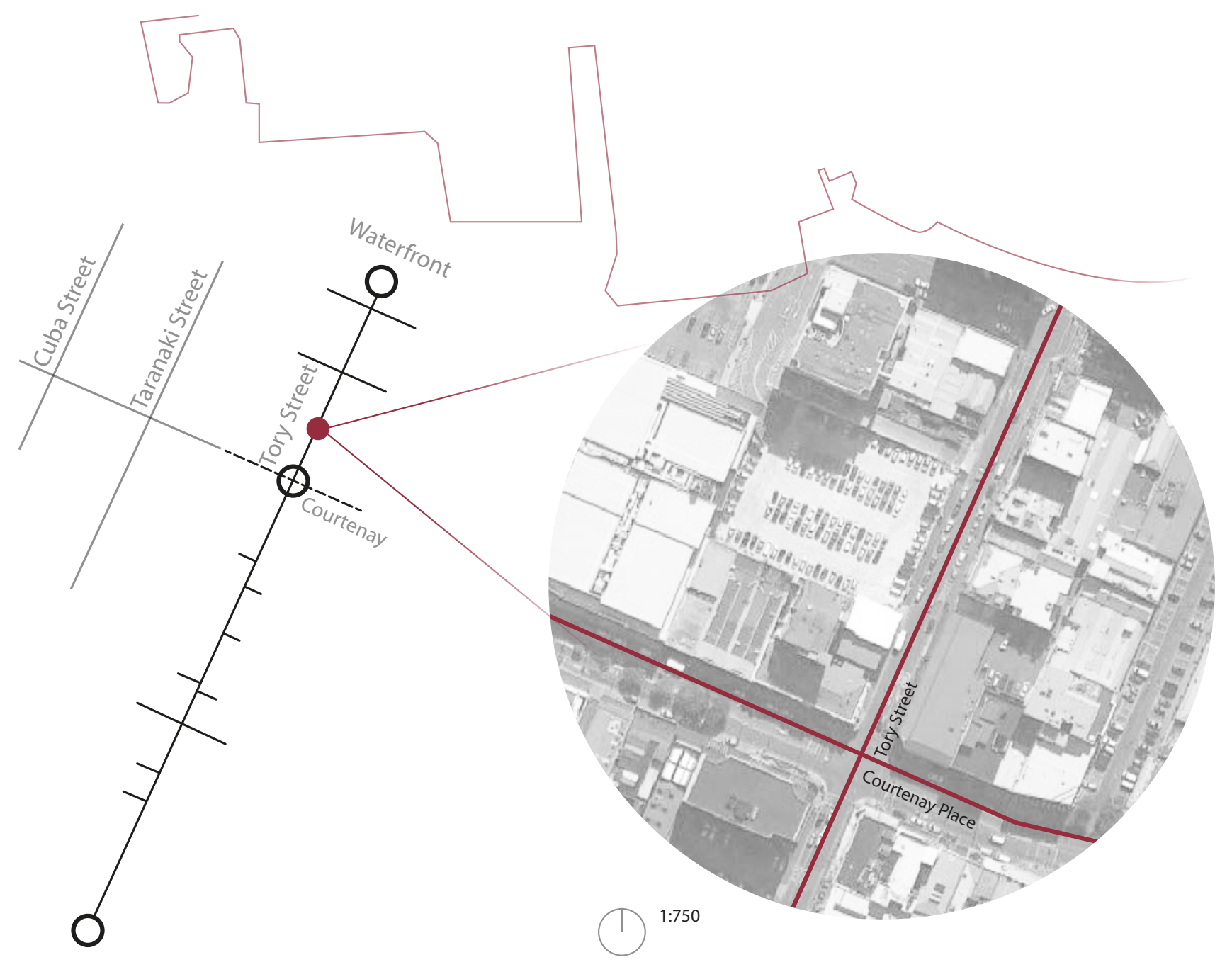

Figure 1.01. Diagram of Tory street in relation to its context. 


\section{Approaching Site}

The site in question is a small portion at the northern end of Tory street, between Courtenay Place and the Wellington Waterfront. The street is fairly normal, with a strip of cafes on the east side of the street and a car park area on the west.

Approaching a street design with a basic comprehension of grasshopper, a natural starting point was to define existing variables and the extents of the site.

The street, like most, was defined by the juxtaposition of the near flat street surface and the verticality of the adjacent buildings. This observation provided the first constraint, the defining bounds in which the final grasshopper script would operate within, any formal operations would not occur beyond these extents. The street, represented in model space as a linear, rectangular plane then led to the question; "how can this be divided spatially?" Naturally it became an experiment of program/ function definition.

Observing the variables of the XY plane, in streetscapes there are two primary elements, the road and the sidewalk. Further exists service access, fire hydrants, street lights, parking, yet these elements are secondary. With this in mind the chicane street became the lead design move. This meant the surface plane in model space could be divided by a pair of curves that represent the street domain and so the chicane became a dividing element. The curves, constrained to the surface plane could be manipulated to provide different variations of street composition. Moving the curves apart from one-another created more street space and potential for parking, moving closer and to a single lane meant more pedestrian space. Applying bends and wiggle patterns to the curves could also provide pockets for pedestrian activity.

On site, photos were taken and drawn over to suggest the placement and movement patterns of digital geometry. This exercise stresses variational potential of the site, looking for the extent an existing element might shift, or the extent of how a new element might operate in the street in relation to existing ones. 


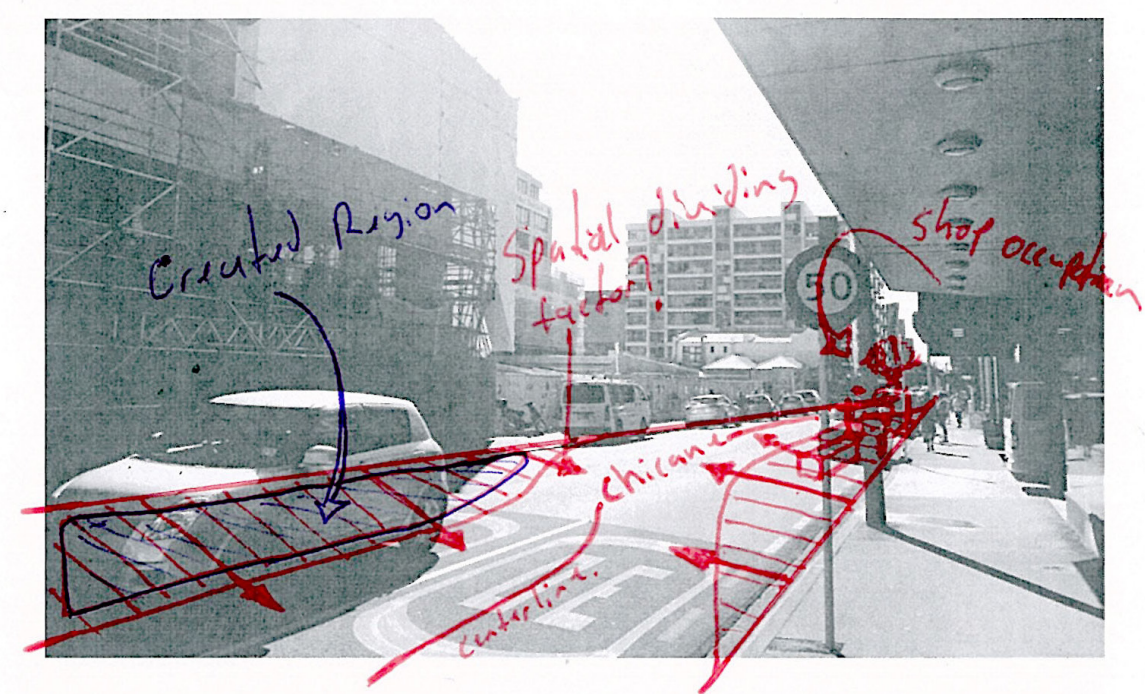

Figure 1.02. Sketch over photo of Tory street. Potential variations

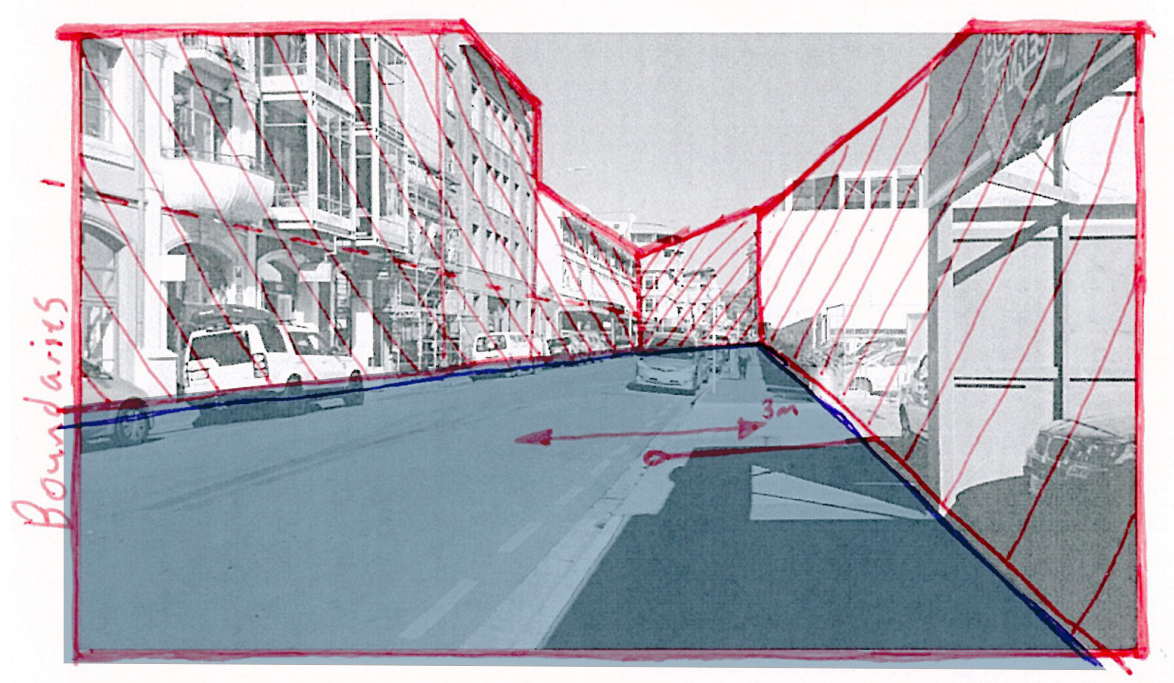

Figure 1.03. Sketch over photo of Tory street. Existing boundaries

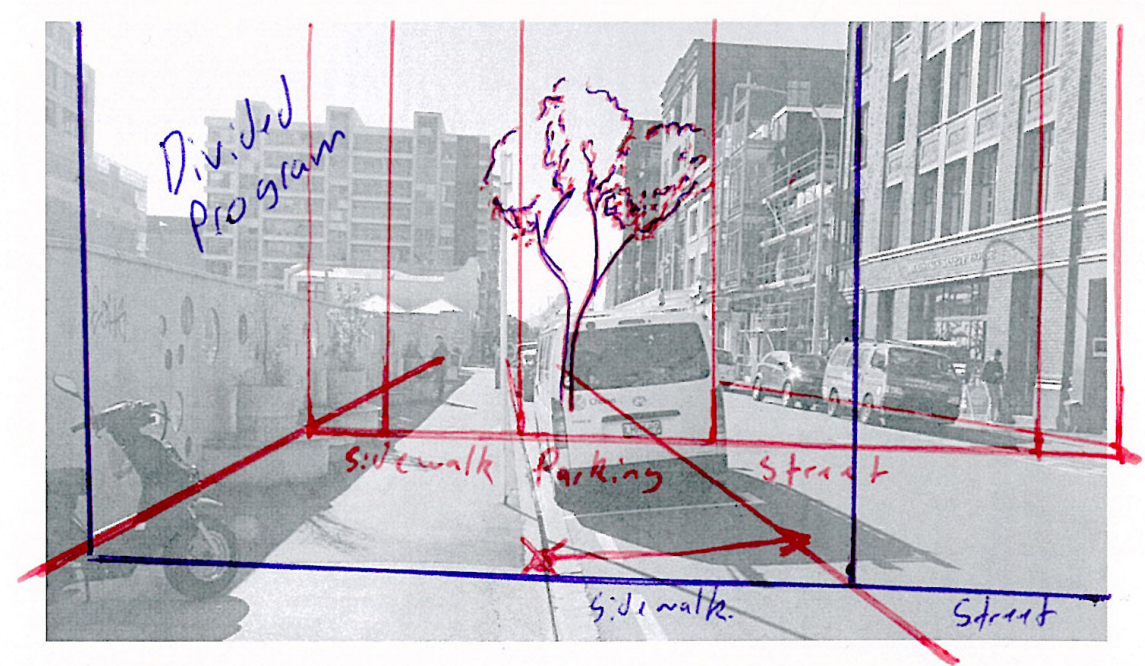

Figure 1.04. Sketch over photo of Tory street. Further potential variations 


\section{The Principal}

The two curves representing the chicane kerb gave definition the idea of the Principal. It was apparent that in order to further the design, each subsequent design move must relate to the preceding, as to maintain a responsive structure and animated model. The principal within its own parameters (Line 1 and line 2) provided constraints to lower logics.

To provide analogy to this concept, a tree's roots might represent the site, the trunk represents the principal, thicker branches represent higher logics and the smaller branches and leaves represent lower logics where detail design might occur.

It is important to note that this study was limited by just one principal logic, the chicane. As will be revealed in more complex relationship building prevalent in the third study of this thesis, multiple principles can occur. However as for single principal relationship structures such as this, the idea of the tree represents the idea well.

Maintaining these relationships means that slight changes might be made to a larger branch and only smaller branches will be effected. Changes to the trunk shifts the entire design, thus making it possible for quick iteration.

\section{Logic Making}

A logic in the context of this thesis, refers to an action or response to the principal or higher logic. They embody the design-move and demand sequential thinking. For instance, with knowledge that the chicane may shift into a variety of different shapes within its local parameters, the first set of logics must recognise and respond appropriately to the change, likely requiring a new parameter setting bounds to the logic locally. A sub-logic responding to this must then define its' own limitations based on this parameter, and so on.

Arguably, this creates a more linear design process. It is important to note here however, the immense flexibility of coding. A clear starting point and method for transitioning scale is defined by the designer andnot restricted within the use of the tool. In the case of this study the chicane at the larger scale provided means to move towards the small scale, each logic moving closer towards detail design. Equally and hypothetically however, if the principal was assigned to a park bench, one might begin to move up in scale, using the orientation of the bench to define formal changes to the context. 


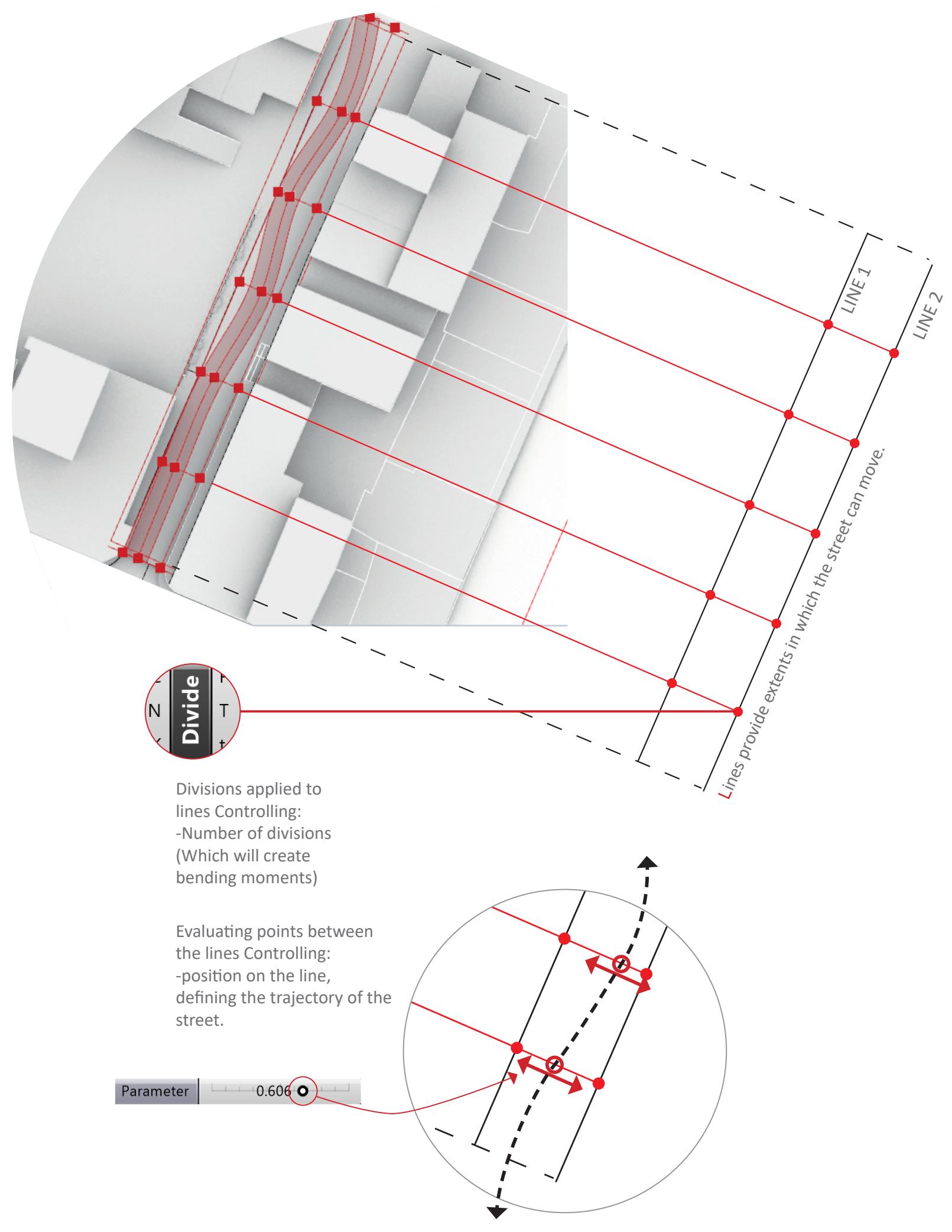

Figure 1.05. Principal logics. 


\section{Sketch 'Recipes'}

Sketching was a crucial tactic to this thesis. Once a certain level of comfortablity was reached with Grasshopper, sketching a plan as to how a script might be built becomes intuitive with sketches such as these.

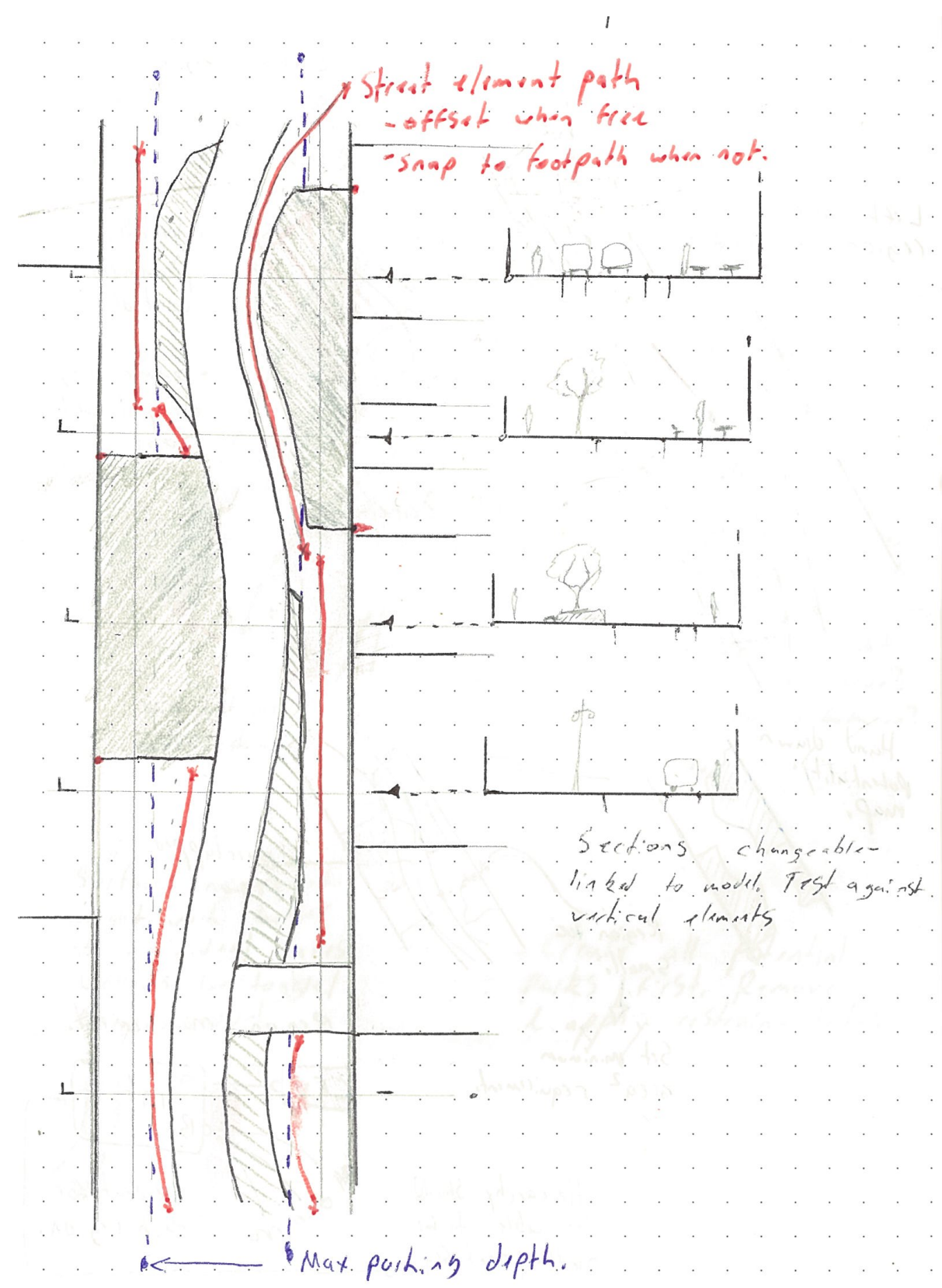

\section{Design Composition}

Figure.1.06 Sketch note, looking at how spatial divisions might be created by bending the street. The blue dash line, outlining the parameter of the chicane principal. The red looking out how curve geometries might be extracted from spatial changes. 

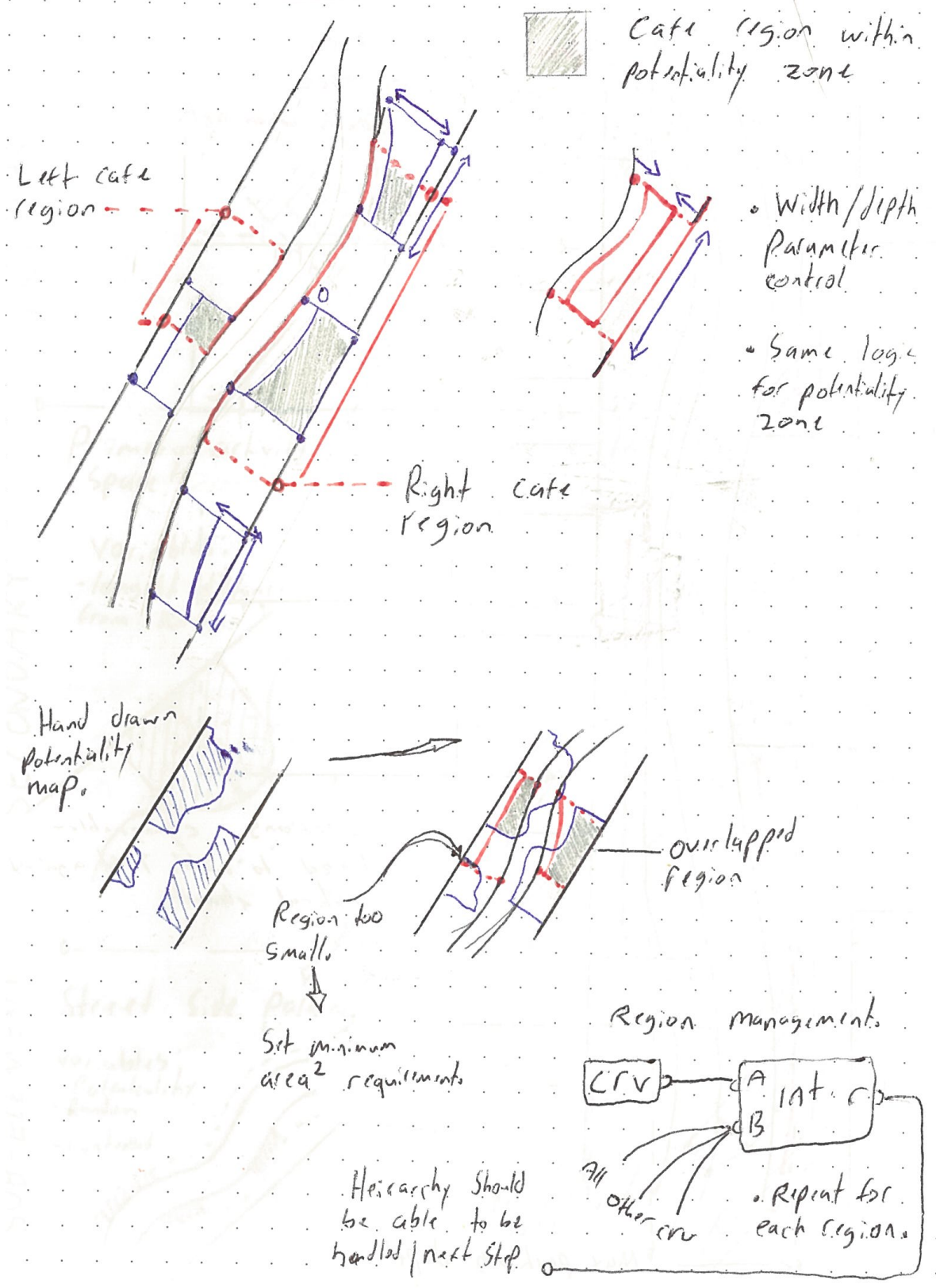

\section{Region Logic}

Figure.1.07 Sketch note, testing region (closed curve) intersections. A GIS, overlay like method that may have aided with form generation. This concept was later not used however. 


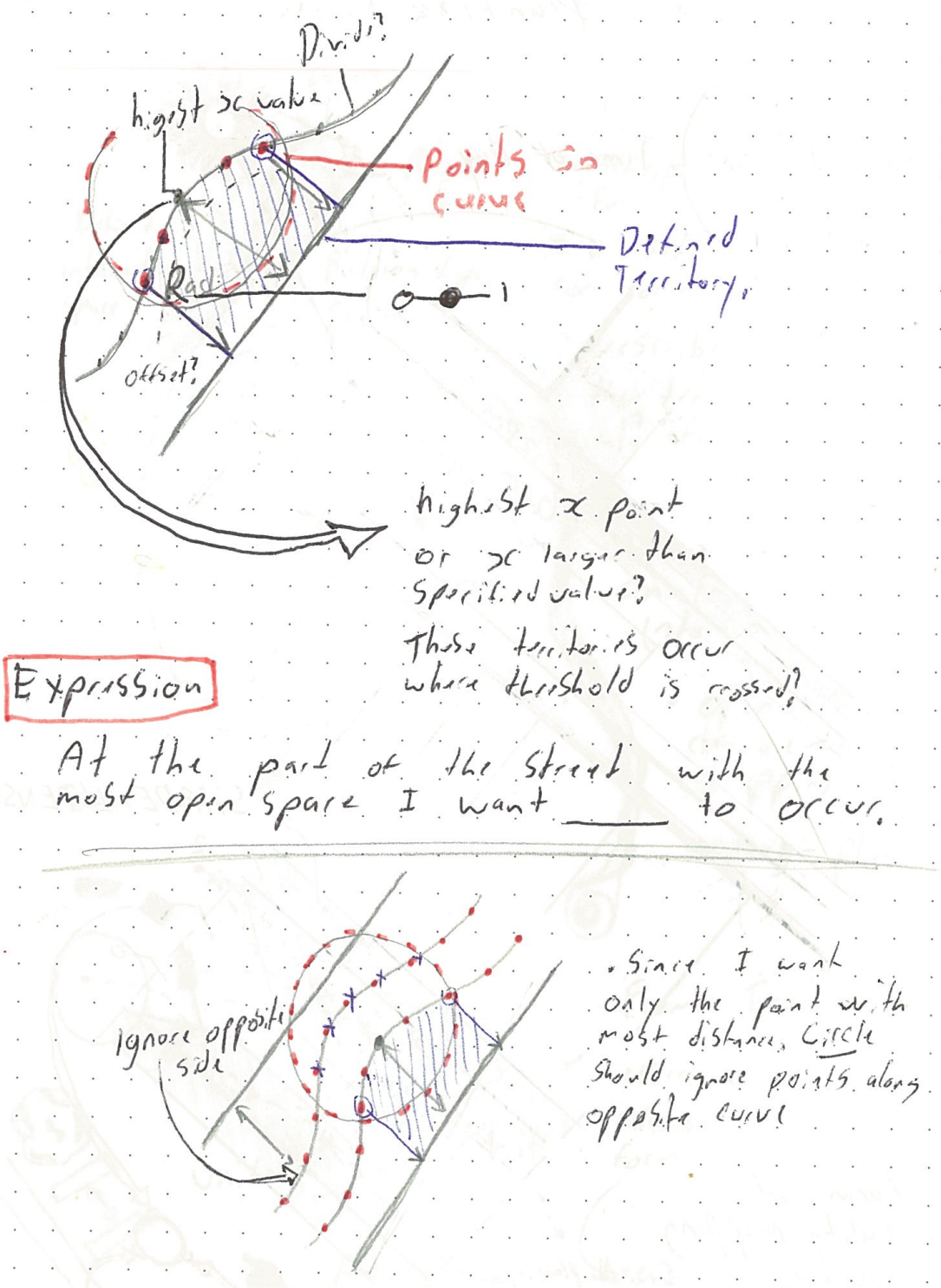

Dynamic Region Logic

Figure.1.08 Sketch note, dynamic region logic. This type of distance based logic was built upon in the third study. 

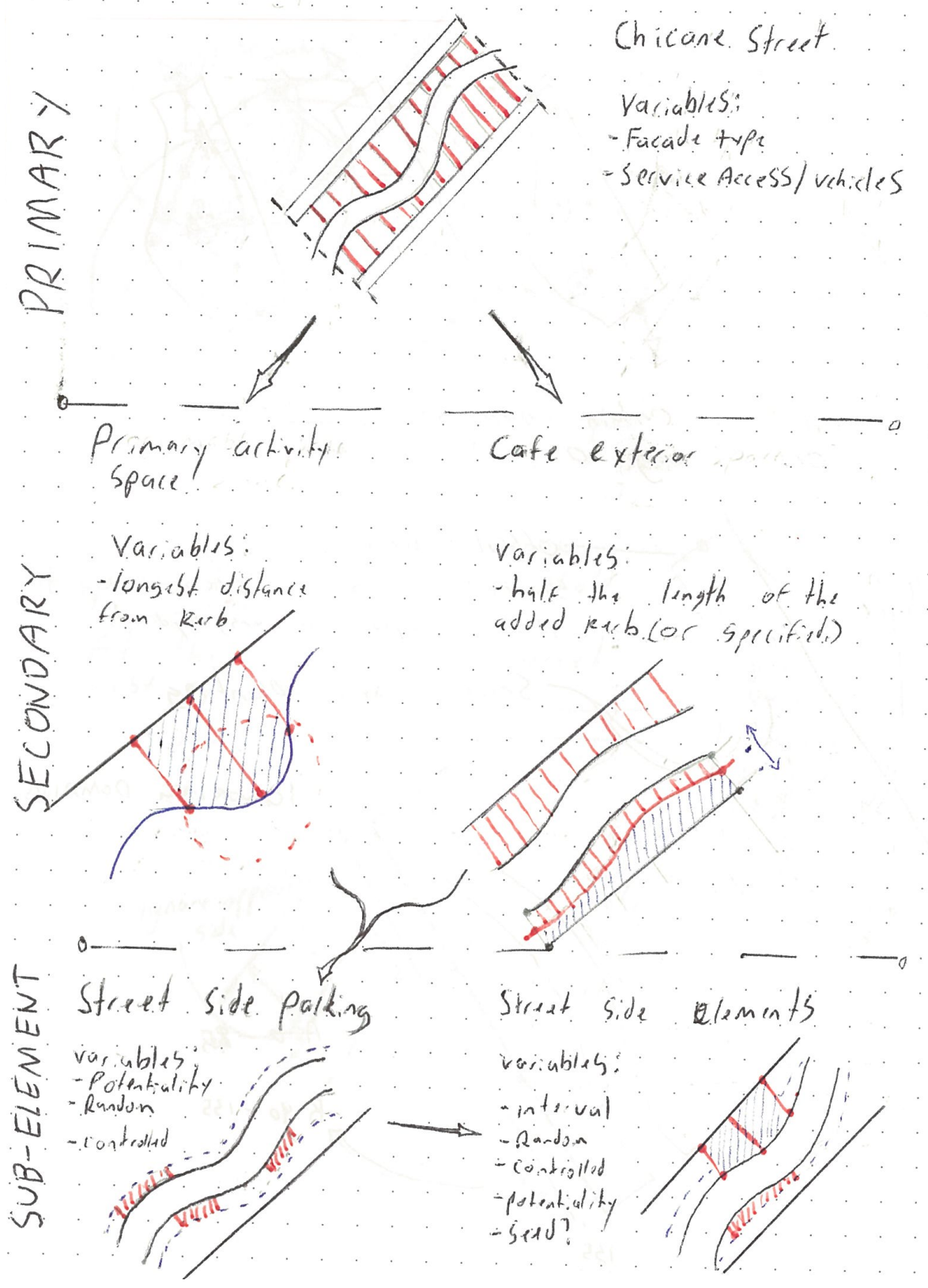

\section{Script Hierarchy}

Figure.1.13 Sketch note, the evolving hierarchy and relationships between design moves. 


\section{Region Logics}

The first logic responding the principal (Chicane street) defined a region that would automatically seek the most amount of space in terms of depth from kerb to site boundary. This asserts that a region will always be placed at this point along the street no matter the shape. This region was used to define program/function space, provide base and to assign constraints to lower logics. For example, parking spaces were removed if they were to intersect with this region. Thus highlighting the workings of the hierarchical structure. The second logic, also defined a region along the street. This one was static, meaning changes to the chicane would not move the region. Instead the region could be defined by the designer, this was important to the site as it allowed for appropriation of this region to be assigned to specific building facades, the line of café buildings for example.

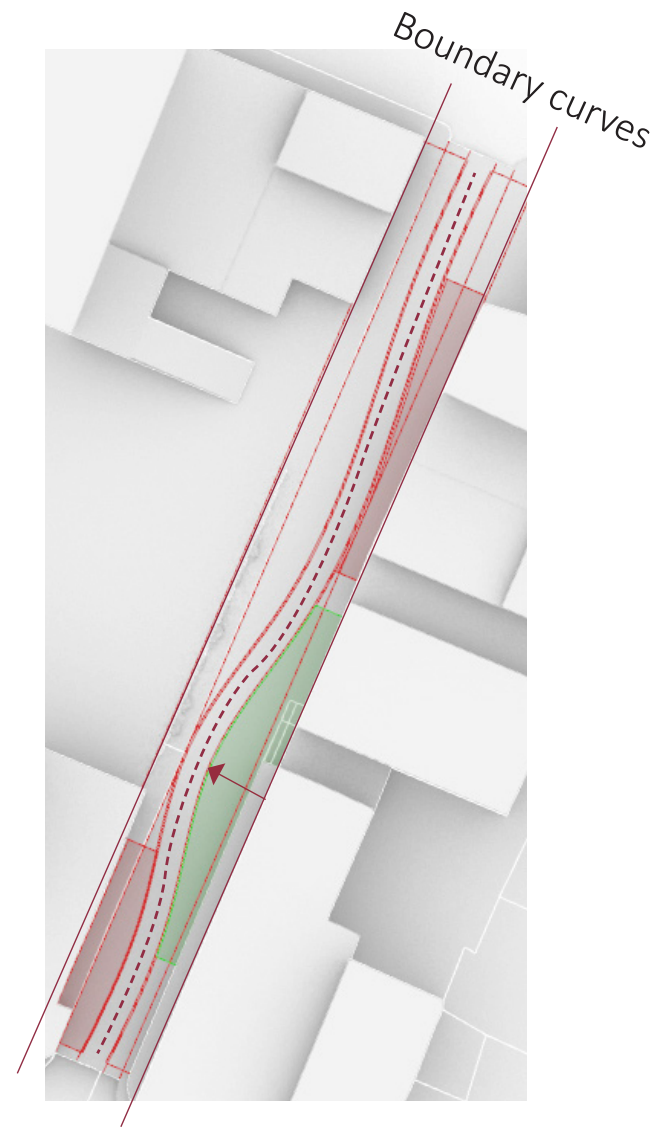

Chicane Variation 1

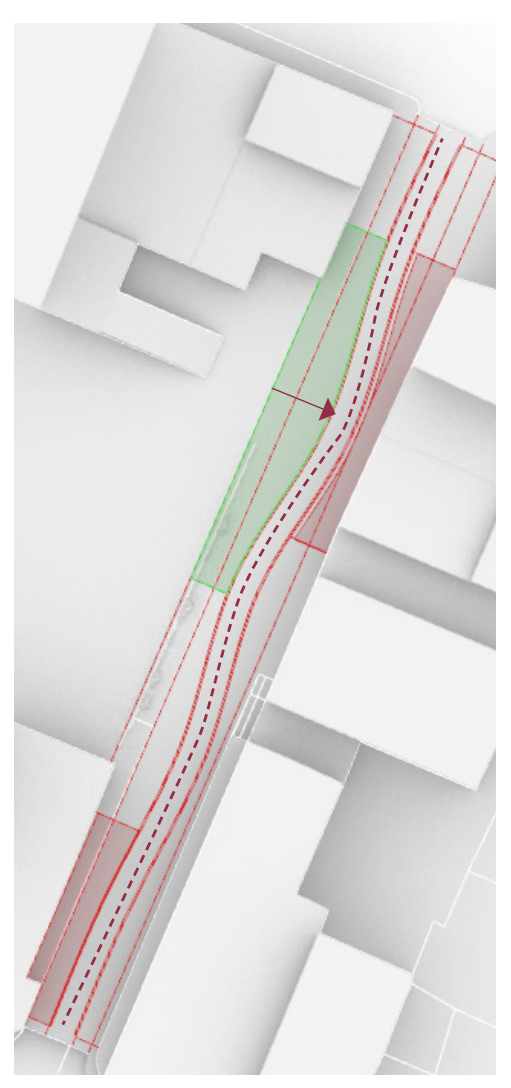

Chicane Variation 2

Figure 1.09. Region logics. Green, dynamic region. Red, Static region. 
Left, red dots restricting area in which dynamic region can appear. It appears at the point of most depth from the curve, within the parameter of the dotted lines. The dotted lines are spaced $1 \mathrm{~m}$ apart. If the dots were enabled along the entire street edge, the dynamic region could appear at any point along the street.

Right, offsets can be applied to the region to lessen the parameter applied to lower logics.

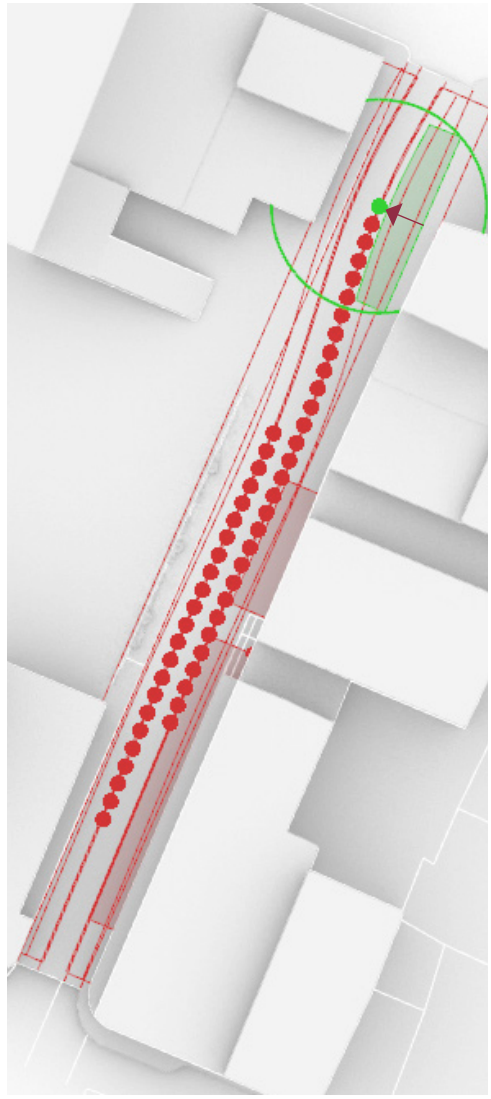

Chicane Variation 1

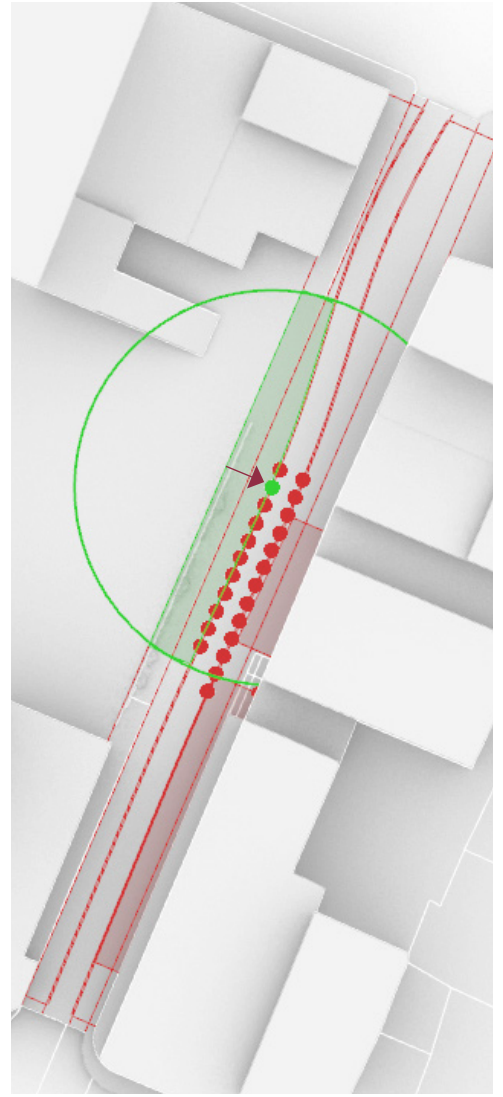

Chicane Variation 2

Figure 1.09. Region logics.

Figure 1.10. Region logic parameters. 


\section{Parking Logic}

Parking spaces were created from an array of rectangles along the street curve. The dimensions of each rectangle was adjustable and set to standard parking dimensions $(3.5 \mathrm{~m}$ by $5 \mathrm{~m}$ ). (The New Zealand Roadmakers Federation, 2012)

Using the intersection between the parking spaces and the spatial regions, parking could be culled based on true-false statements. This meant priority was assigned higher to the regions, therefore when an intersection was true, the parking would be removed. Alternatively, the parking could have been used to reduce the size of the regions.

In a landscape architectural sense, this would imply a level of significance applied to the parking. As more value was placed upon the regions as functional pedestrian spaces; and not the parking, the values of the designer and their favouritism towards pedestrian space is carried throughout the scripting process.

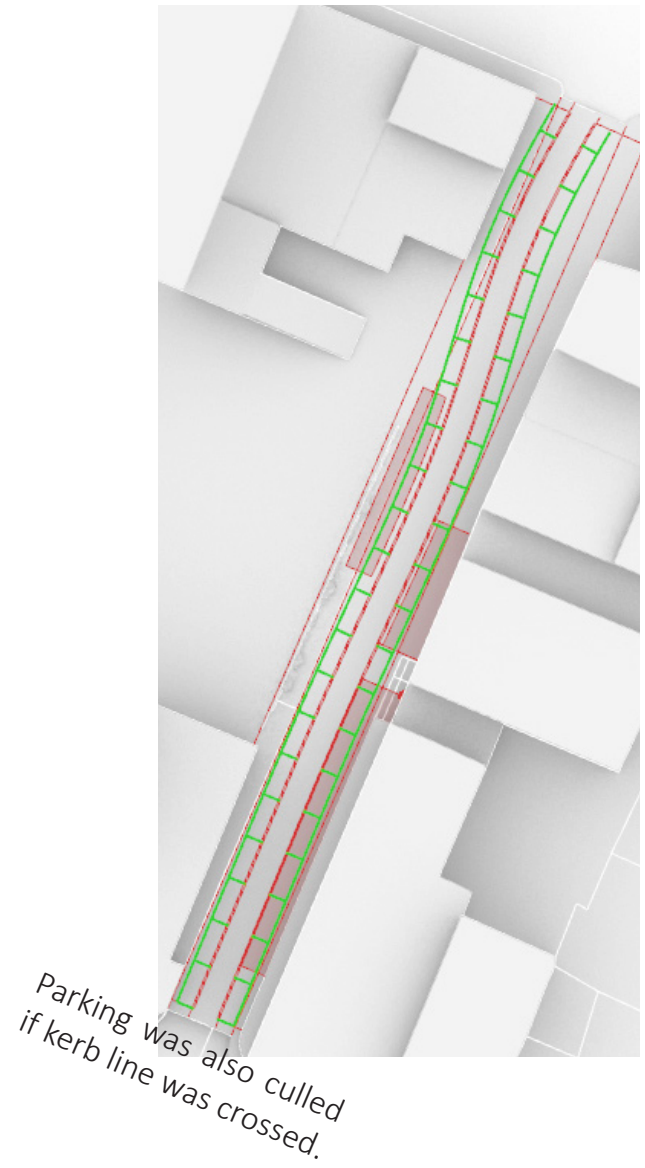

Chicane Variation 1

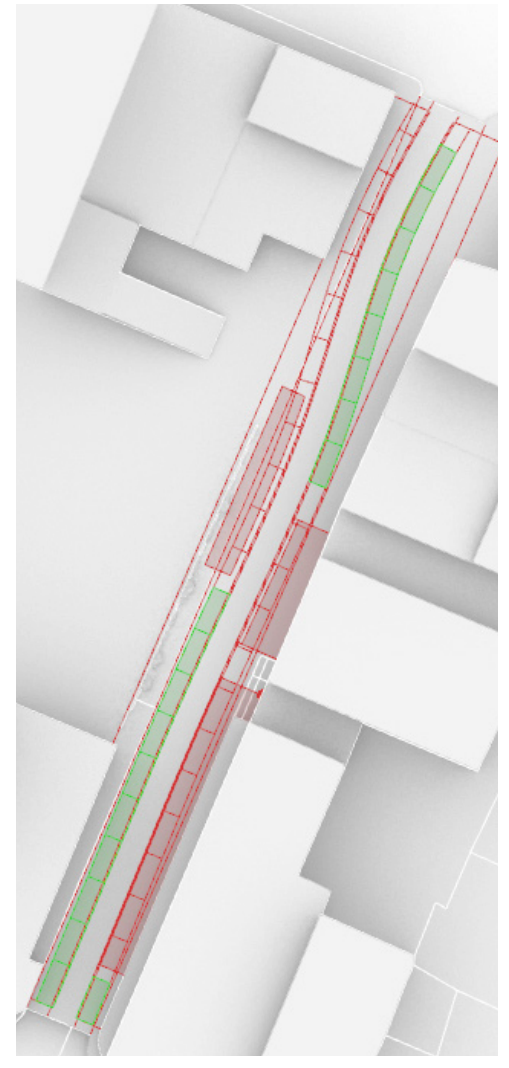

Chicane Variation 2

Figure 1.11. Parking Logics. Green, dynamic region. Red, Static region. Left, arrayed rectangles (in green) representing parking.

Right, Cull operation applied. 


\section{Street Side Elements}

Considering the program regions and parking as the core features of the street, the street side elements were given lower priority than both of these logics.

This made for a simple strategy for element generation where leftover space between the regions and parking was assessed, providing a base to array a set of lines in which an element could appear.

The regions and parking therefore became the parameters for park benches, street lights and trees.

The generated lines were then grouped by proximity with one another. Then counted for their group size, a number sequence was then assigned to generate combinations of vertical elements and benches. For example a 0 might represent no generation, a 1 might generate $1 \mathrm{~m}$ of bench, with two 1's generating $2 \mathrm{~m}$ of bench, and a 3 might generate a vertical element. This type of generation design is; by nature, responding to the linear order of the line groups and certainly sacrifices design finesse for generative efficiency.

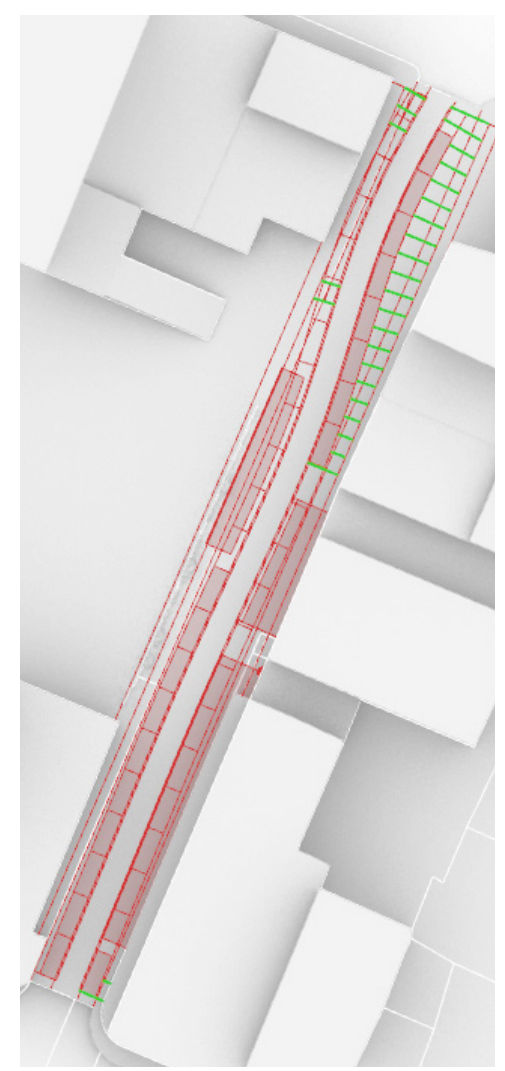

Chicane Variation 1

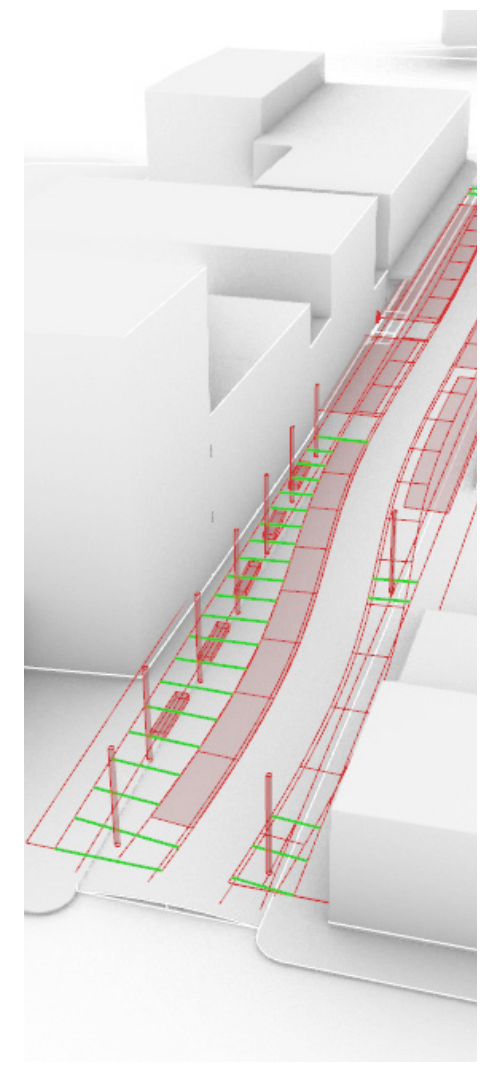

Chicane Variation 2

Figure 1.12. Street side logics. Left, Line assessment applied. Right, Seating and vertical elements generated. 


\section{Further Sketch Notes}
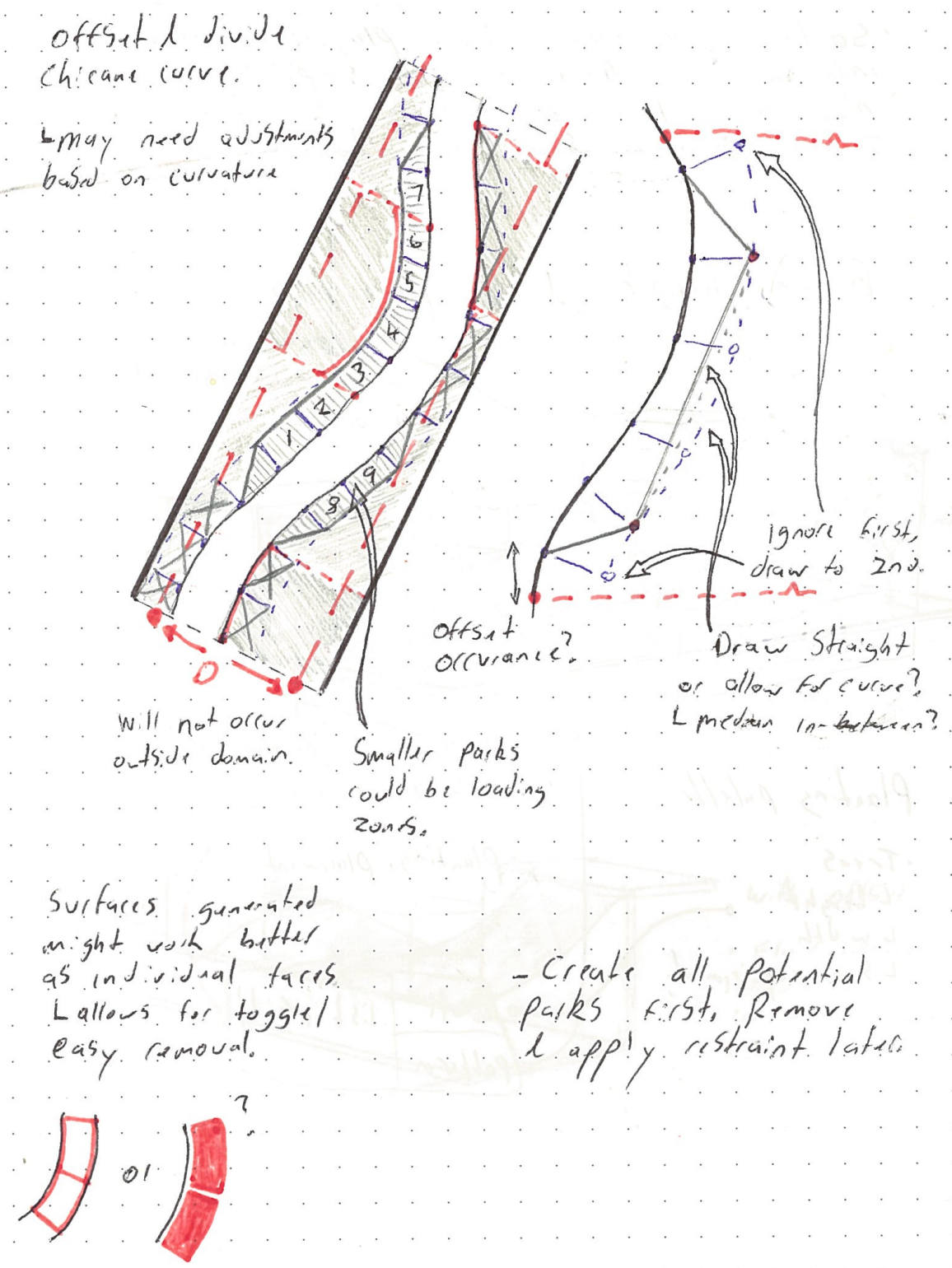

Parking Logic

Figure.1.14. Sketch note, testing logic behind parking generation. 


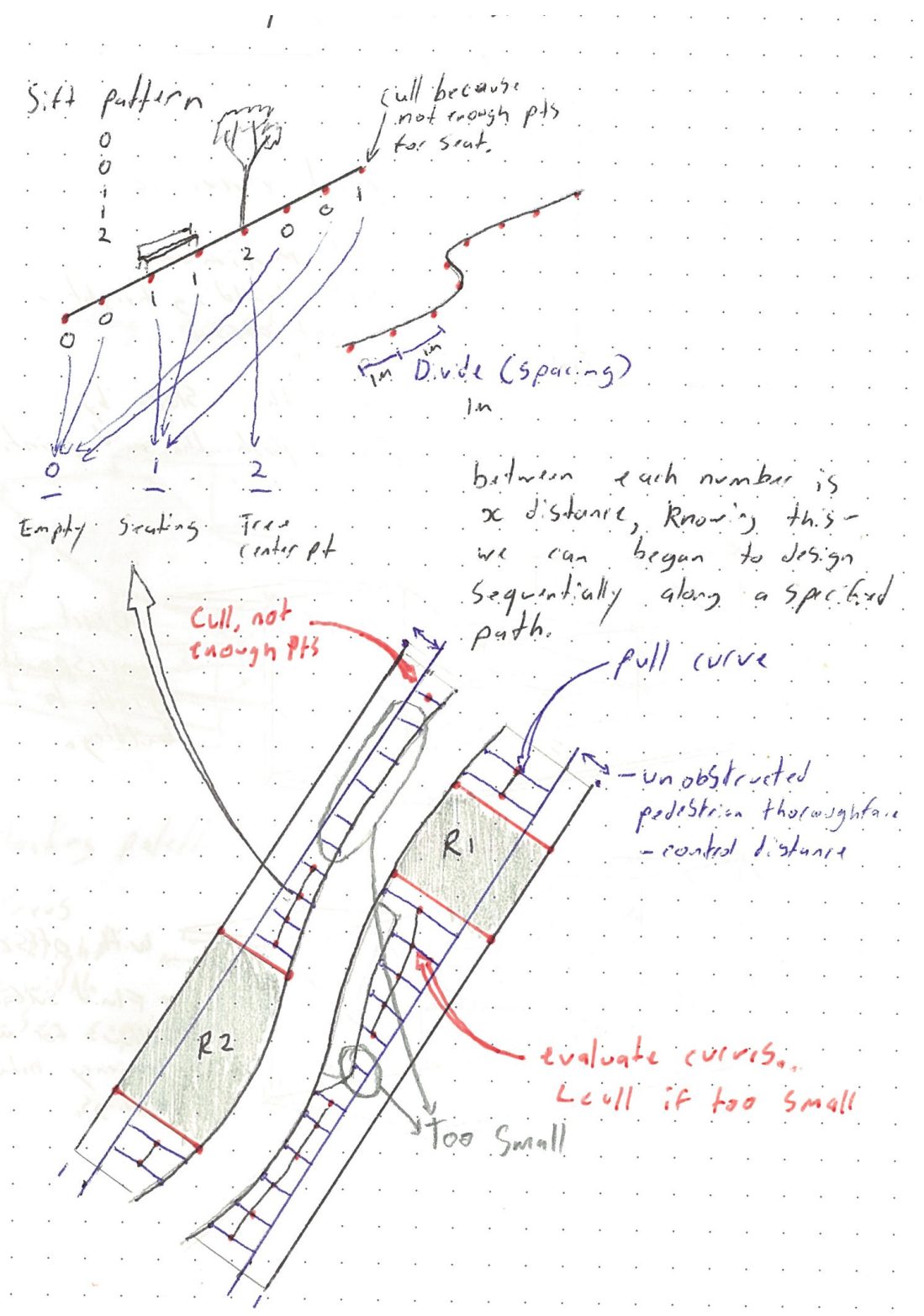

\section{Street Side Elements}

Figure.1.15. Sketch note, testing logic for assessing free space and number sequencing for generation. 


\section{$Z$ value variables}

- So far sitevariubles ha-e plogyed exclusively into the $x y$ plane as this simplitios the algorithm in troms of building $3 d$ form Some $z$ axis parametess. Should bi introdued.

Brilding heights $l$ z parameters.

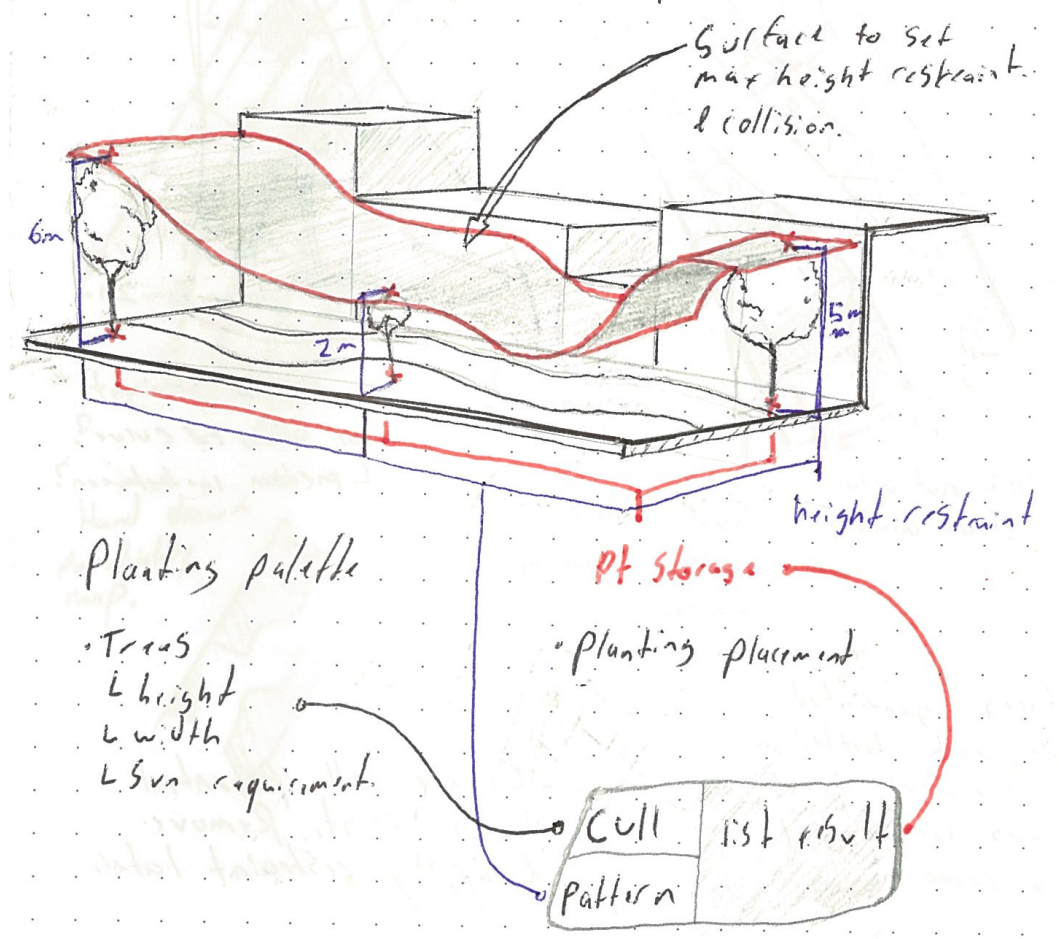

Height Limit Parameter

Figure.1.16. Sketch note, a curved surface was used as an applied exterior parameter for the vertical elements. Restricting their height limits while allowing for variation in this regard. 

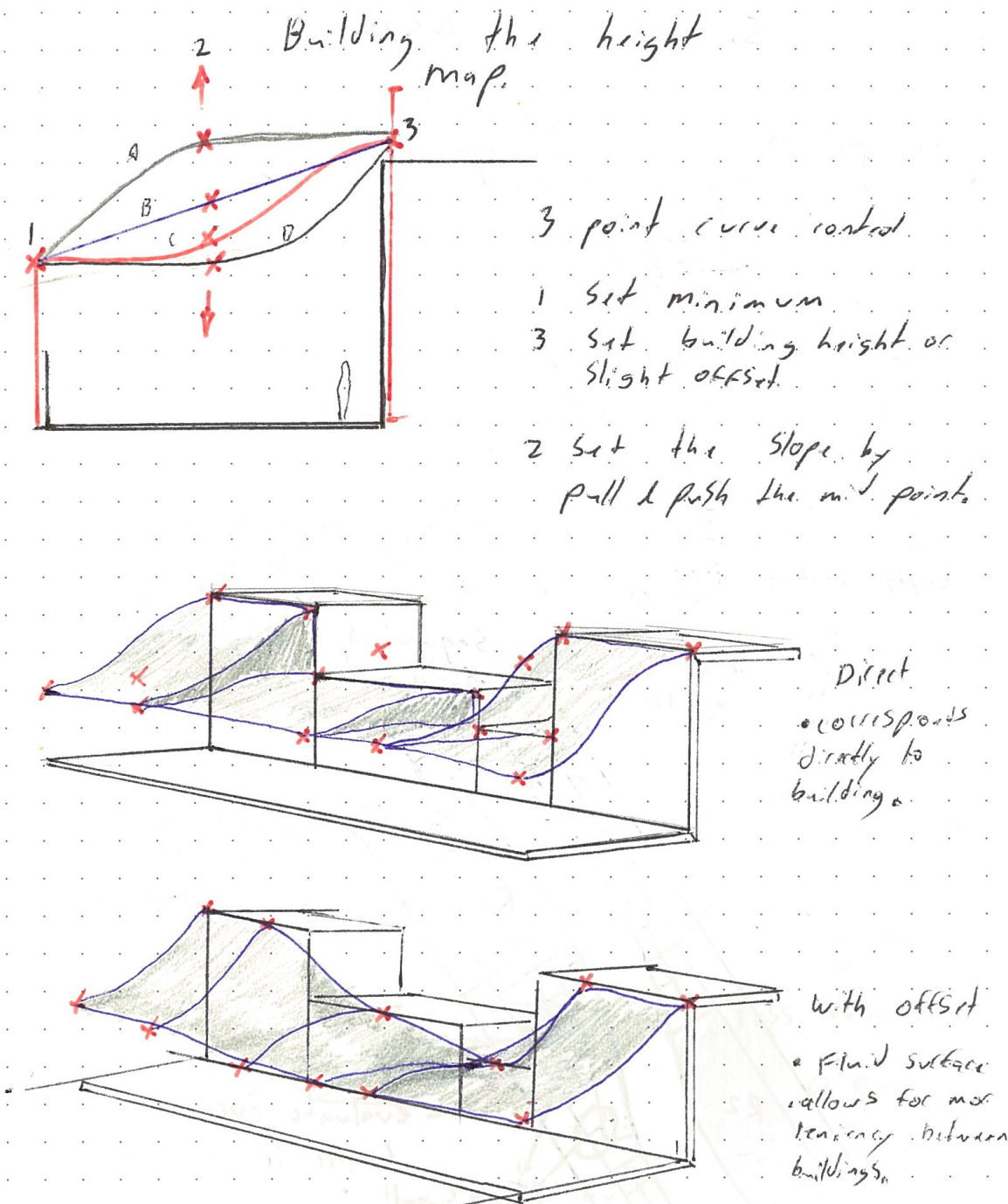

Height Limit Parameter 2

Figure.1.17. Sketch note, an alternative method for creating the surface. It was important to maintain the best control, as height limits for elements occurring on either side of the street was to be considered independently. The final model used a hybrid method of the two sketches. 


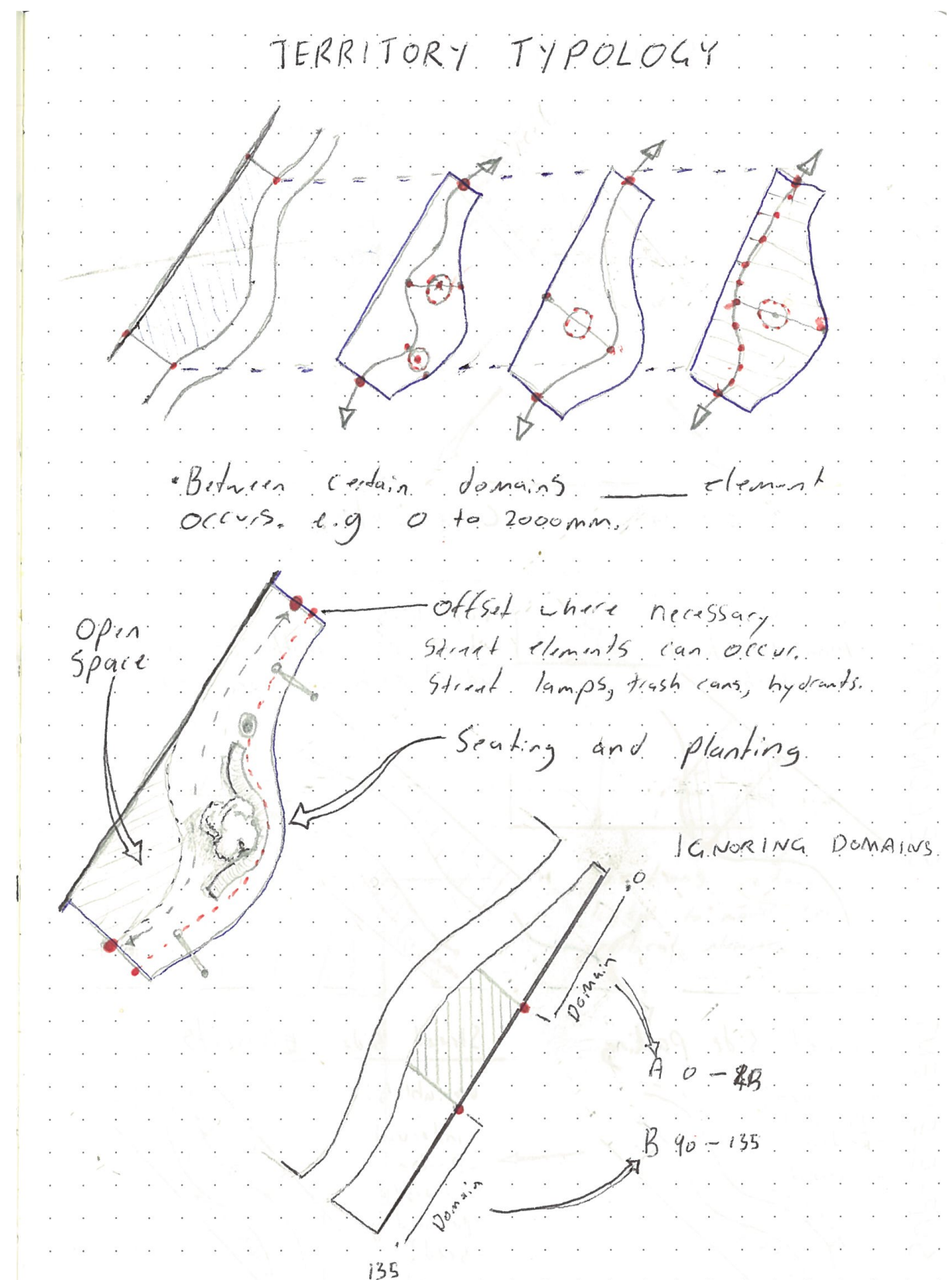

Regions potential for further development.

Figure.1.18. Sketch note, although not further explored, looking at how the regions might be developed further in detail, further spatial divisions and generation methods. 


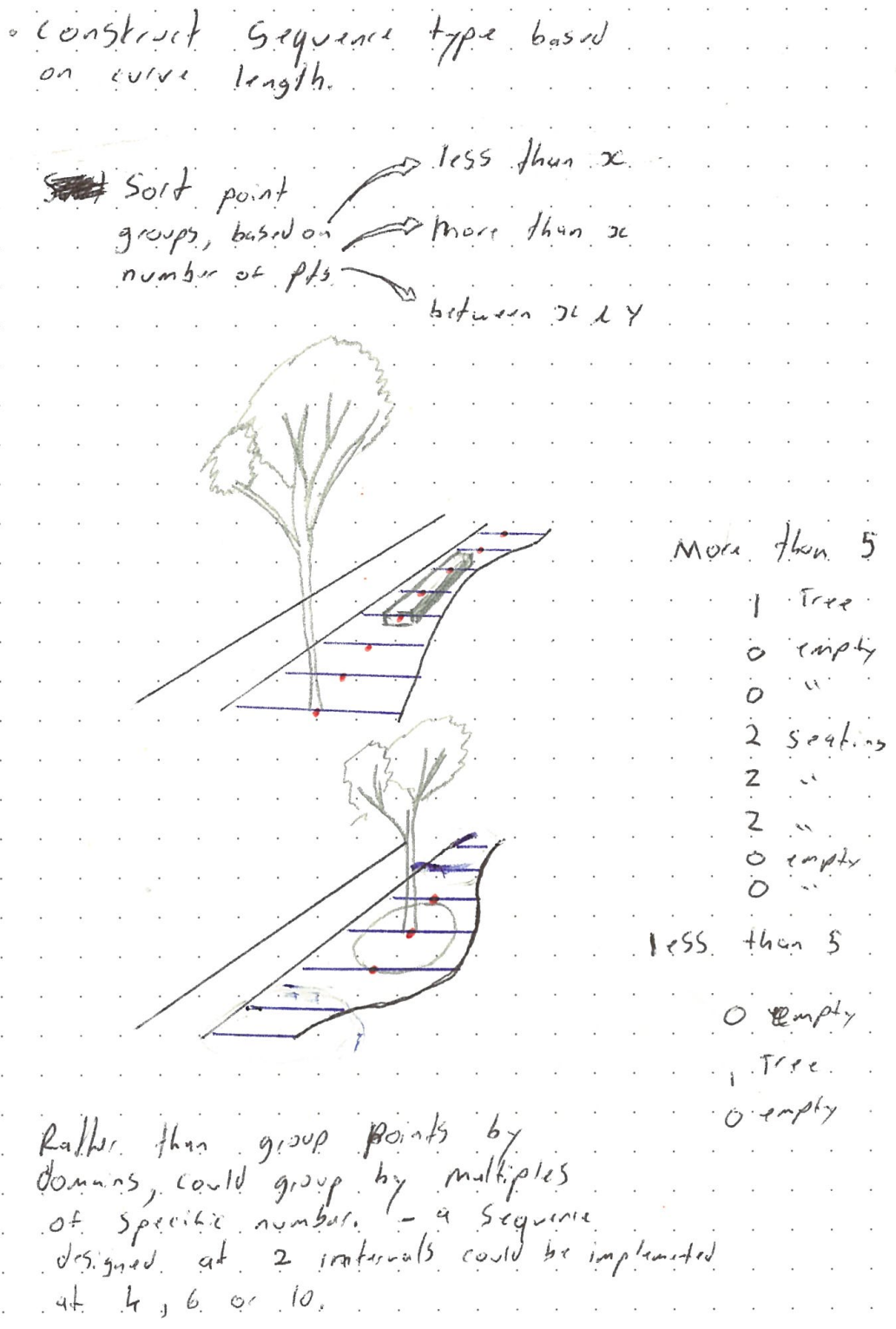

\section{Sequence Generation}

Figure.1.15. Sketch note, Further tests on number sequencing. 


\section{A Conceptual Machine}

The final script was formed from the planning of sequential and scaling relationships between geometries. The hierarchy of logics enabled a flow on effect of shifting parameters. An animated model and spatial generator of streetscape conditions was the result.

From a practical standpoint, this type of modelling enables a level of stress testing. With the freedom to shift each logic, altering and tweaking to respond in a required manner, the model might then be used to test specific scenarios and as a tool of spatial interrogation. For instance, spatial combinations might be explored providing different outcomes, applying favour towards certain elements. Such as searching for possibilities while retaining a specific number of parking spaces, or looking at how pedestrian space might be made through applying higher variations of bending moments to the chicane.

This might also provide a better communication tool for client engagement, as real-time alterations can be made to the concept. 


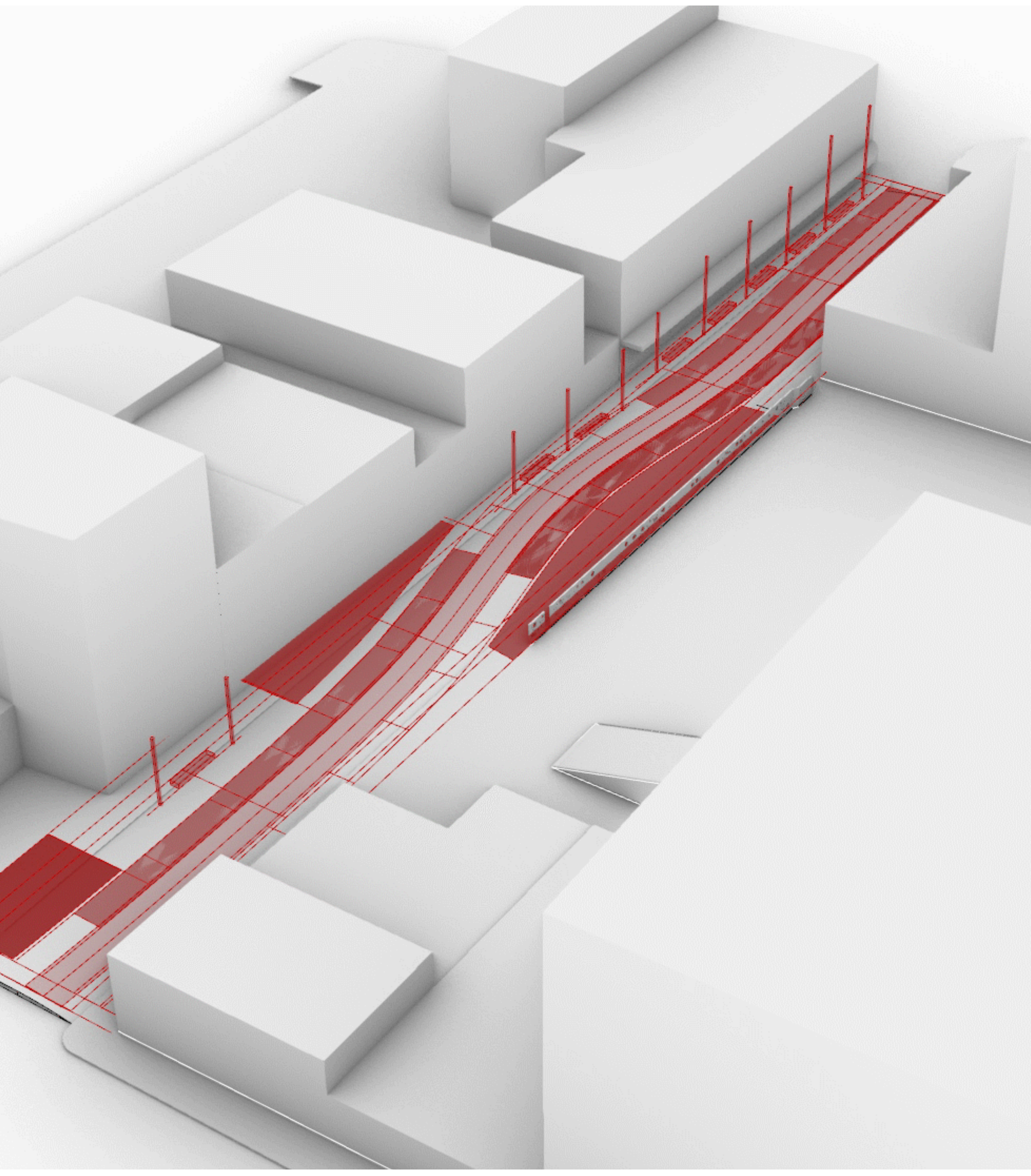




\section{Script Interface}

The script grew sequentially from the principal curve geometries. These three figures show each logic in order, briefly discussing their interface and parameters.
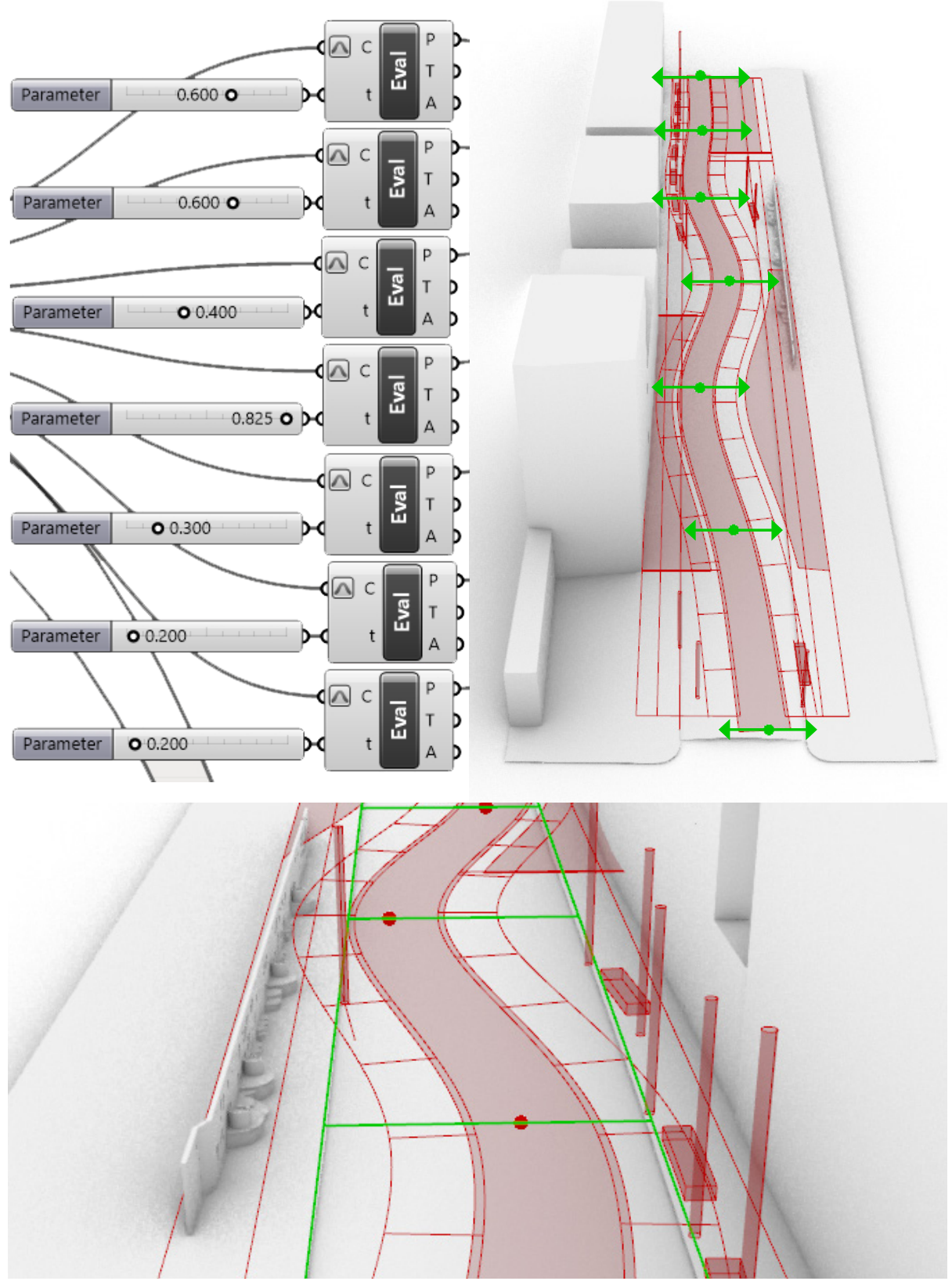

Chicane / Principal

Figure.1.20. The principal chicane curve operated on sliders. Top showing the movement points, below showing the extents the chicane can move. 


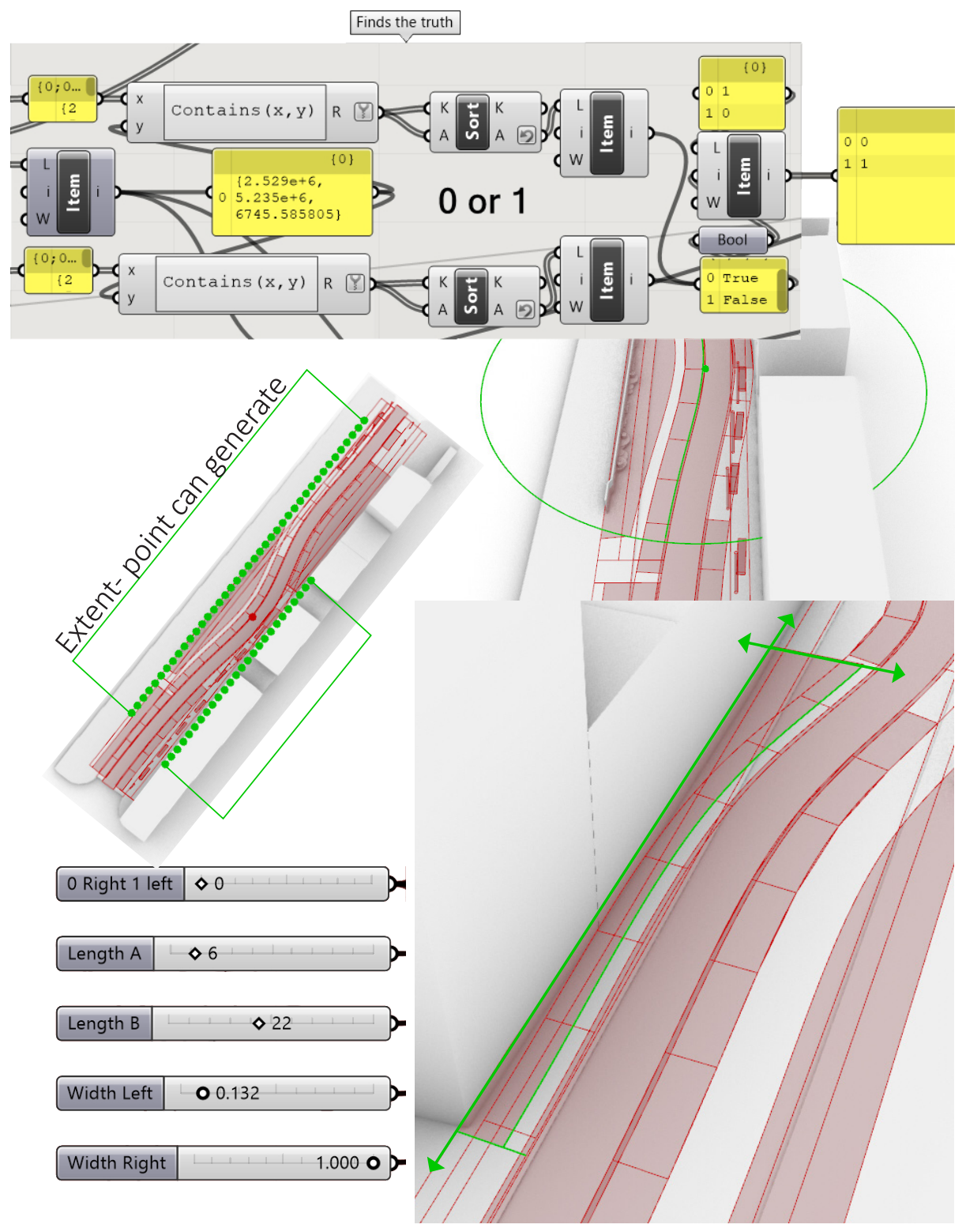

Logics \& Regions

Figure.1.21. Top displaying the dynamic region, the script used to find which side the point occured on, and the extents that point can generate in. Bottom displaying how the regions' size can be adjusted. 


\section{Making Variations}

By shifting parameters compositions could be generated. Each test looked at variations, favouring either parking, pedestrian space or a mix.
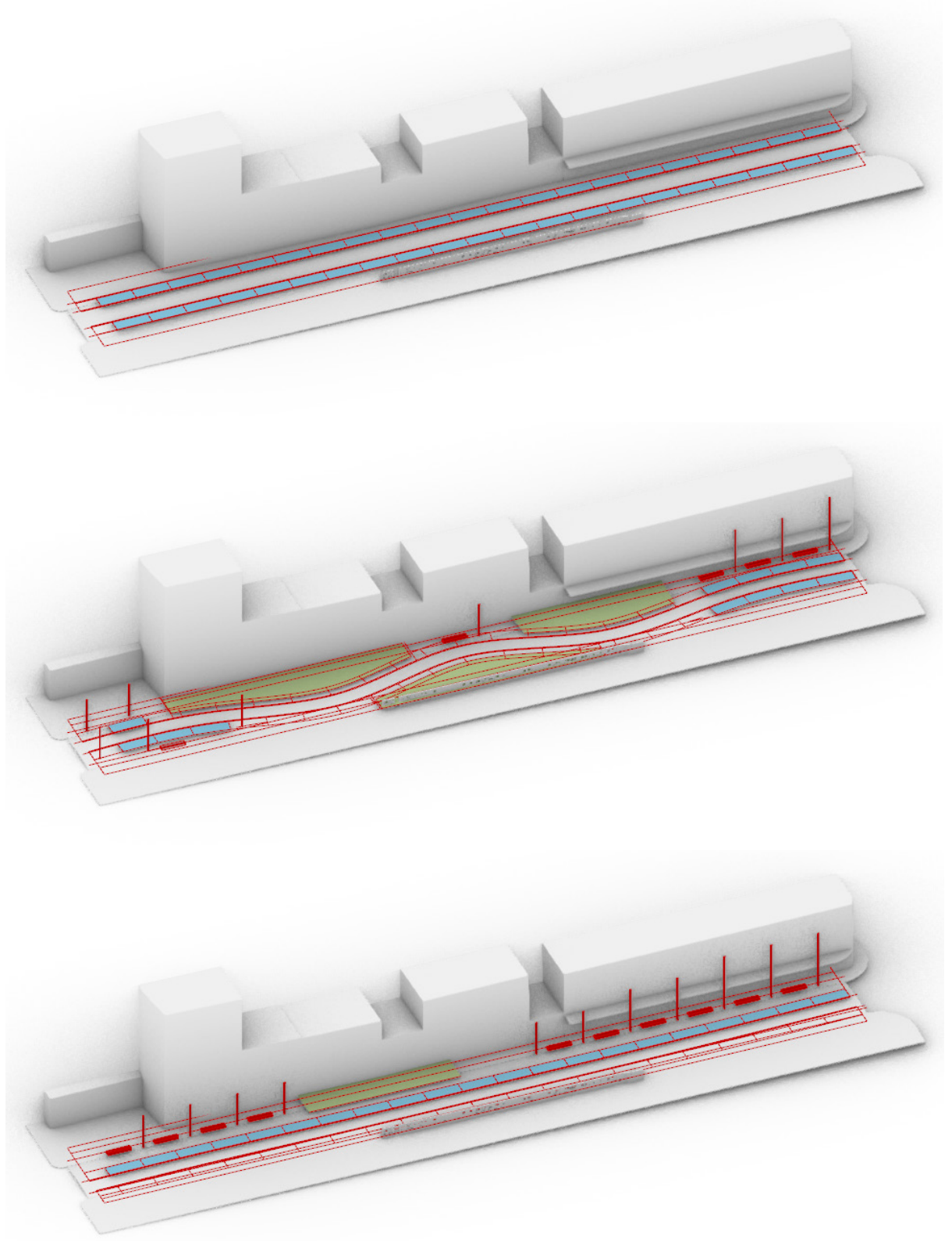

Street Side elements

Social Space

$\square$ Vehicle Service

Figure.1.22. Variations produced from the conceptual tool. 

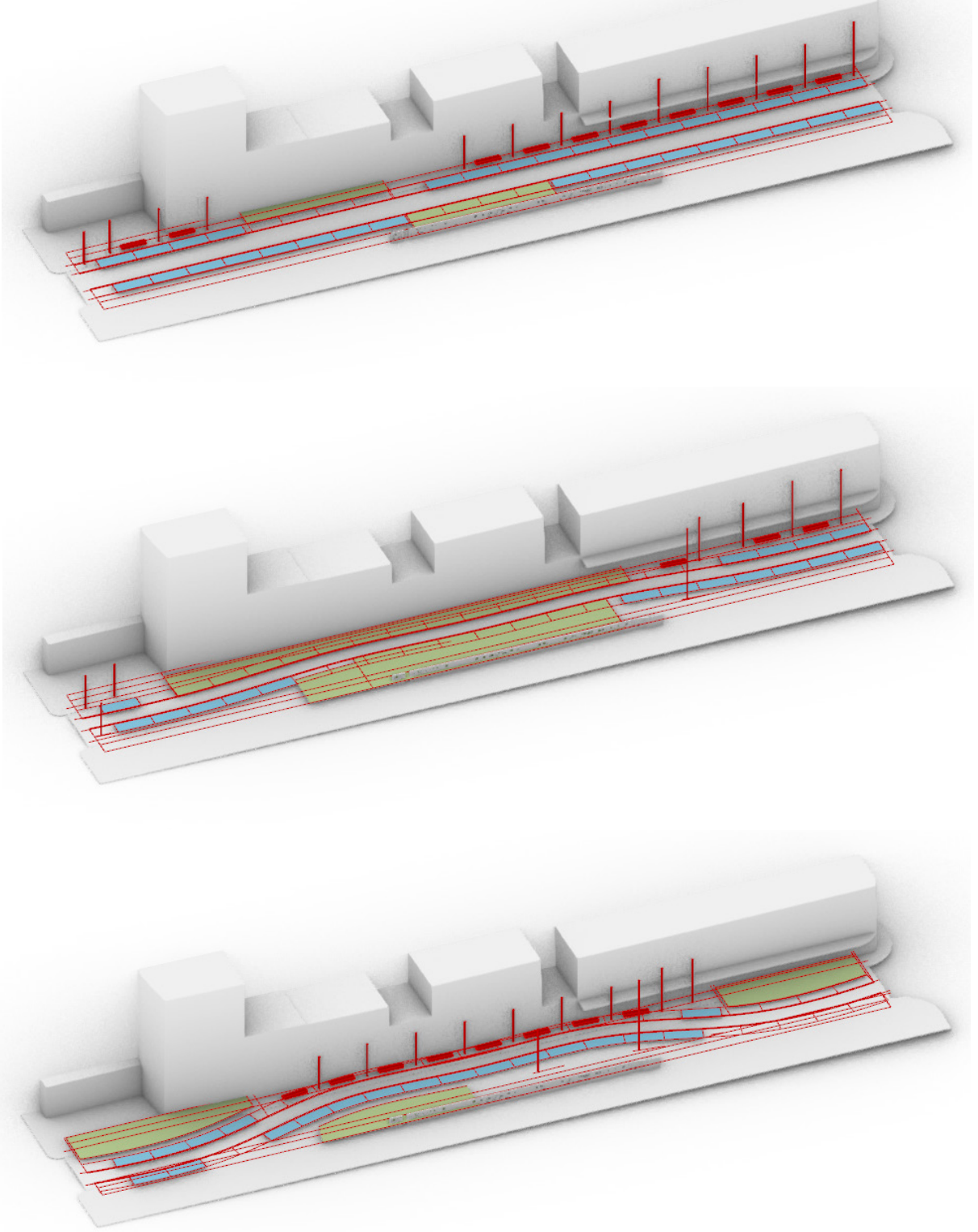

Figure.1.22.1. Variations produced from the conceptual tool. 

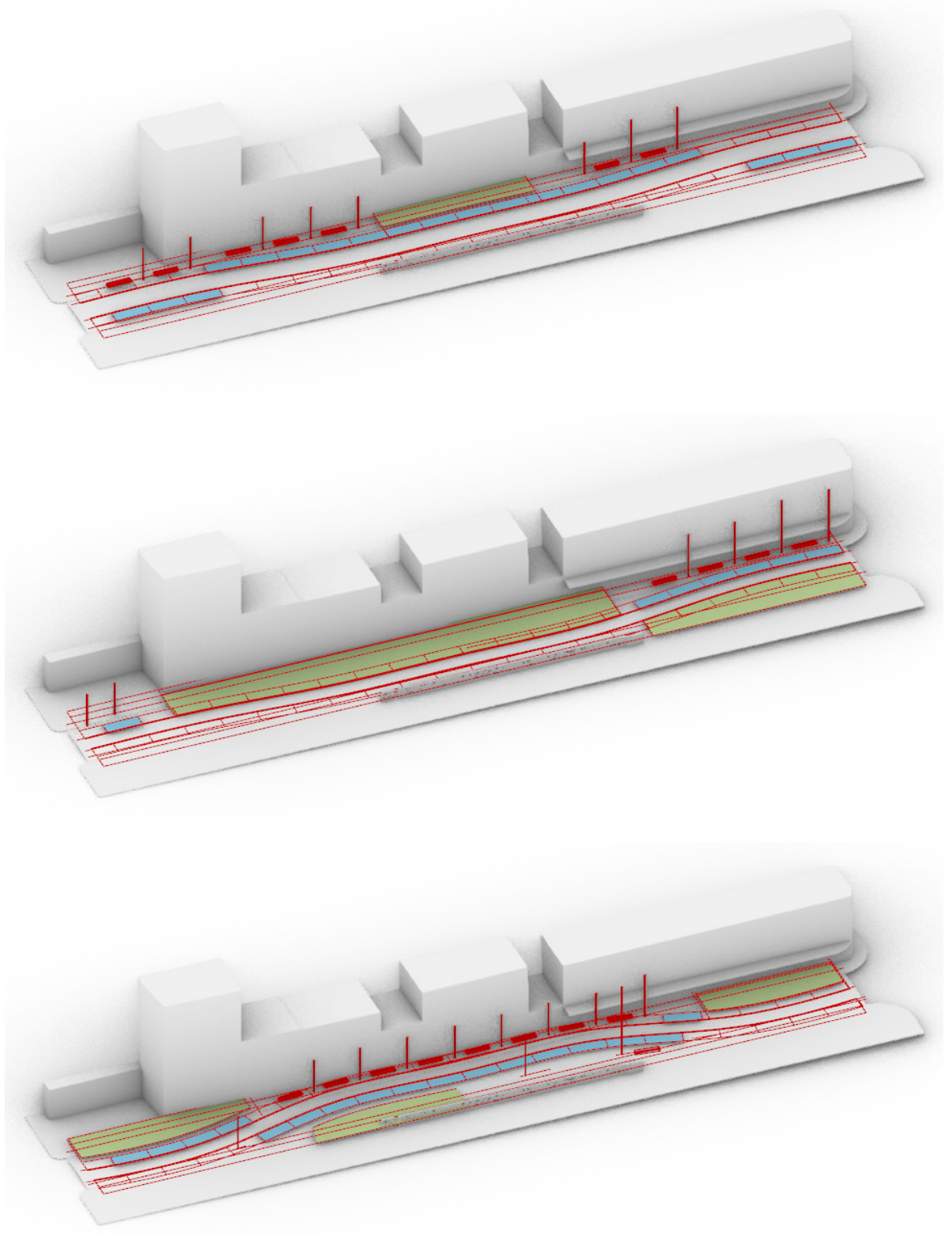

Figure.1.22.2. Variations produced from the conceptual tool.

Page 46 Study 1 

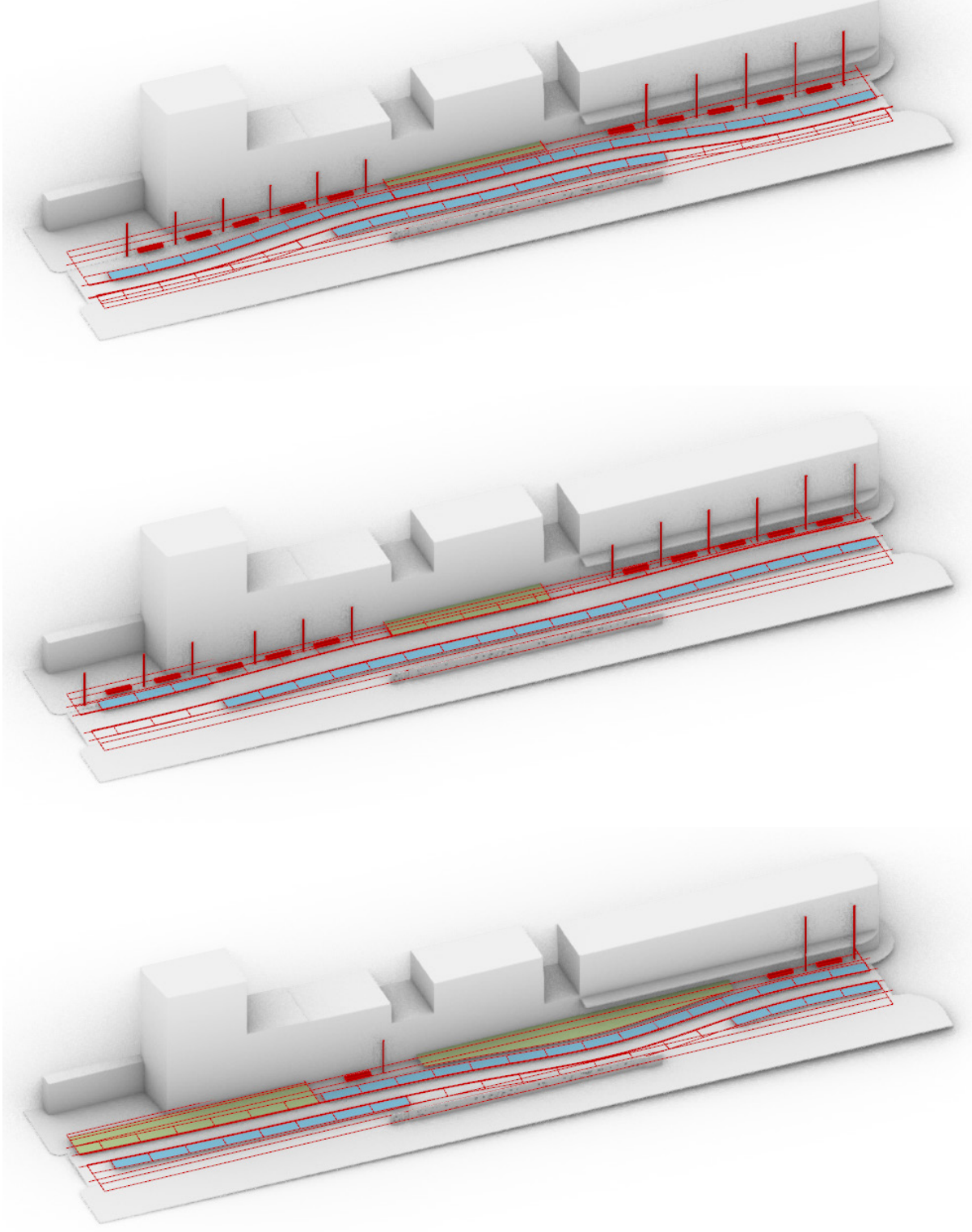

Figure.1.22.3. Variations produced from the conceptual tool. 


\section{Site Variables}

To further test the tool, variations produced on the following page responded to these conditions.

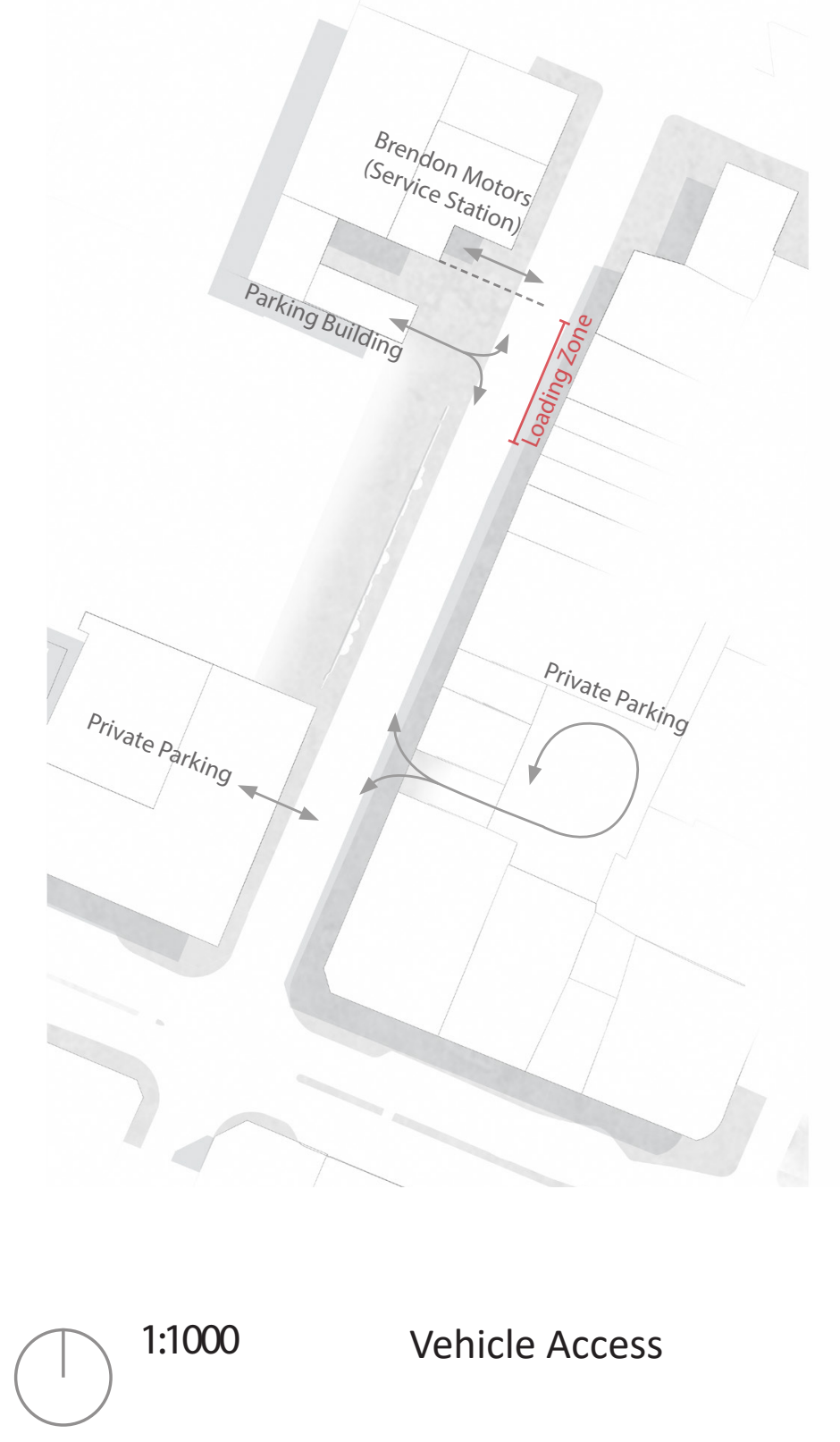

Figure.1.24. Tory street, vehicle access.

Page 48 Study 1 


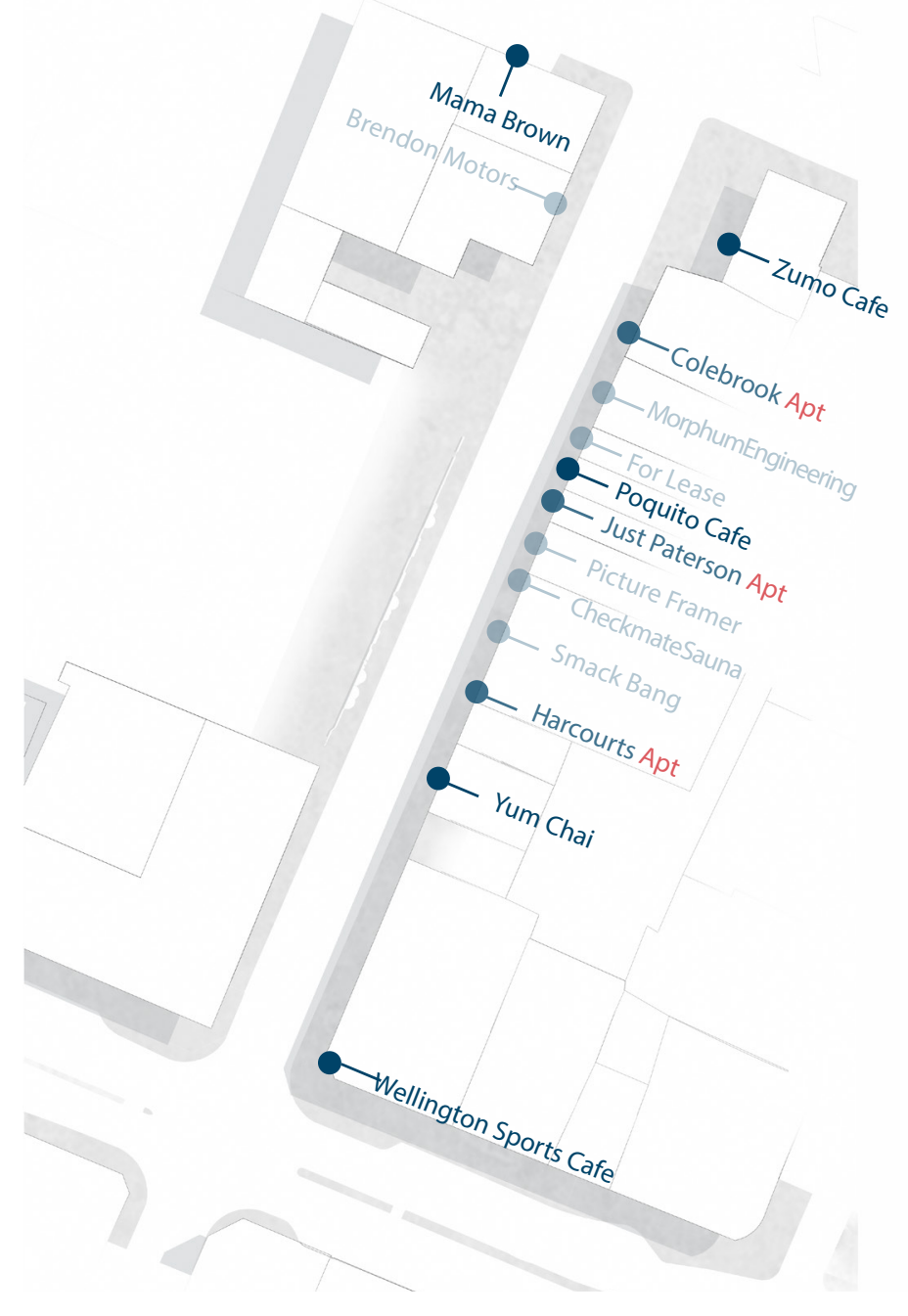

Facade Type

High Activation (Cafes and Resteraunts)
Mid Activation (Apartments / Residential)
Low Activation (Niche Stores, offices and
vacant lots)

Figure.1.25. Tory street, façades. 


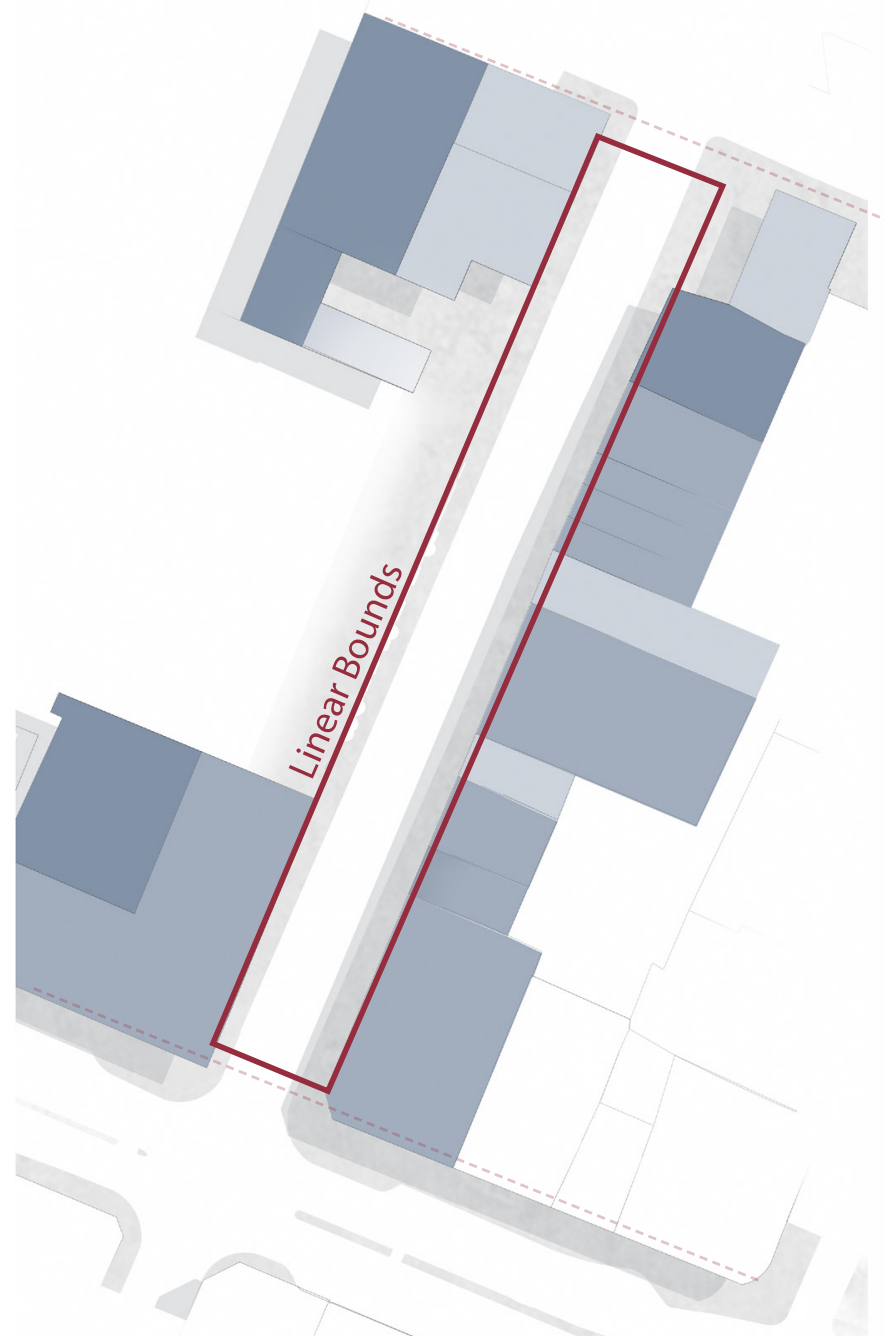

Building Height

20+

10 to $19 \mathrm{~m}$

1 to $9 \mathrm{~m}$

Figure.1.26. Tory street, building heights.

Page 50 Study 1 
Page intentionally left blank | Page 51 

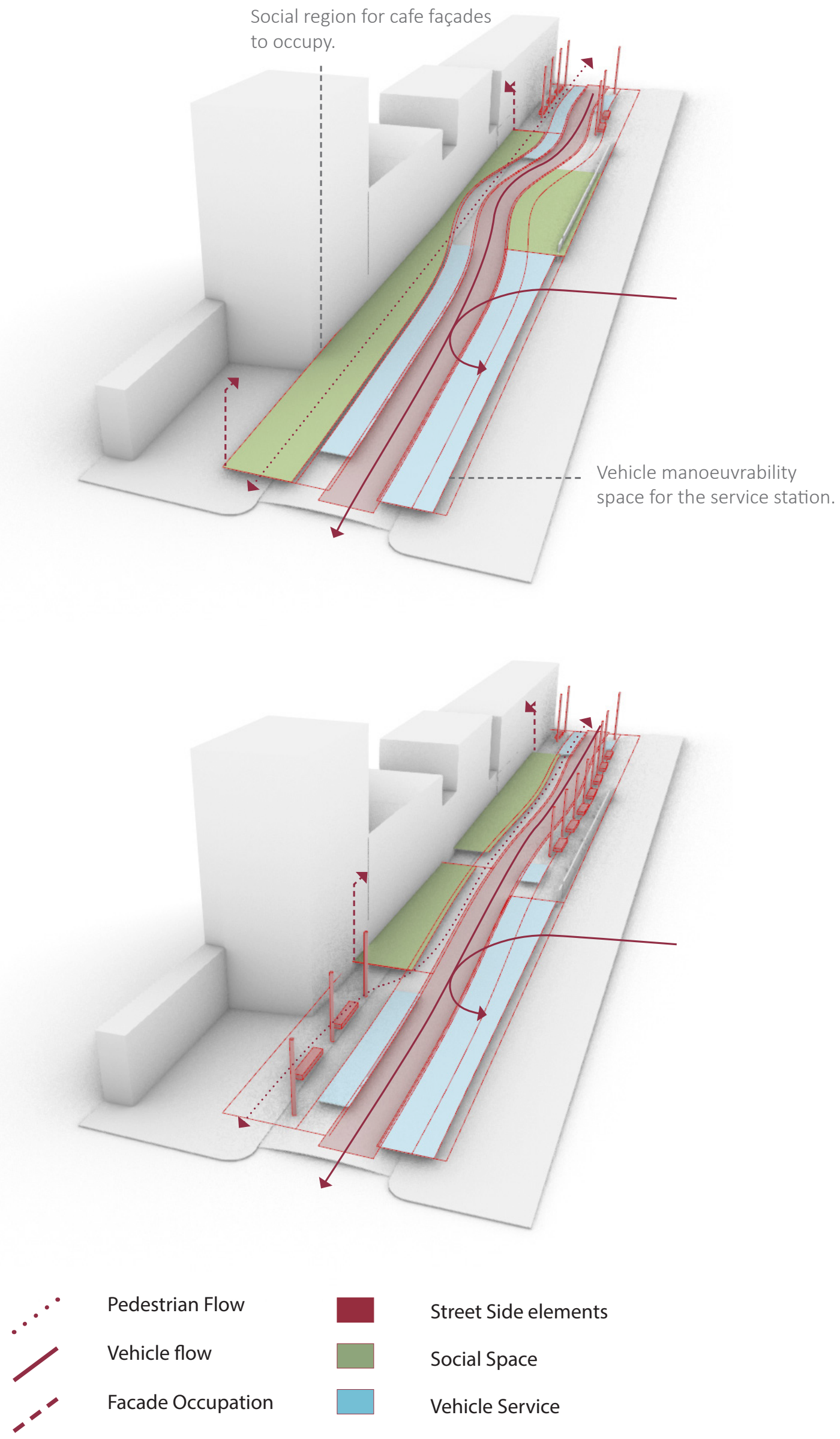

Figure.1.25. Tory street, vehicle access. 

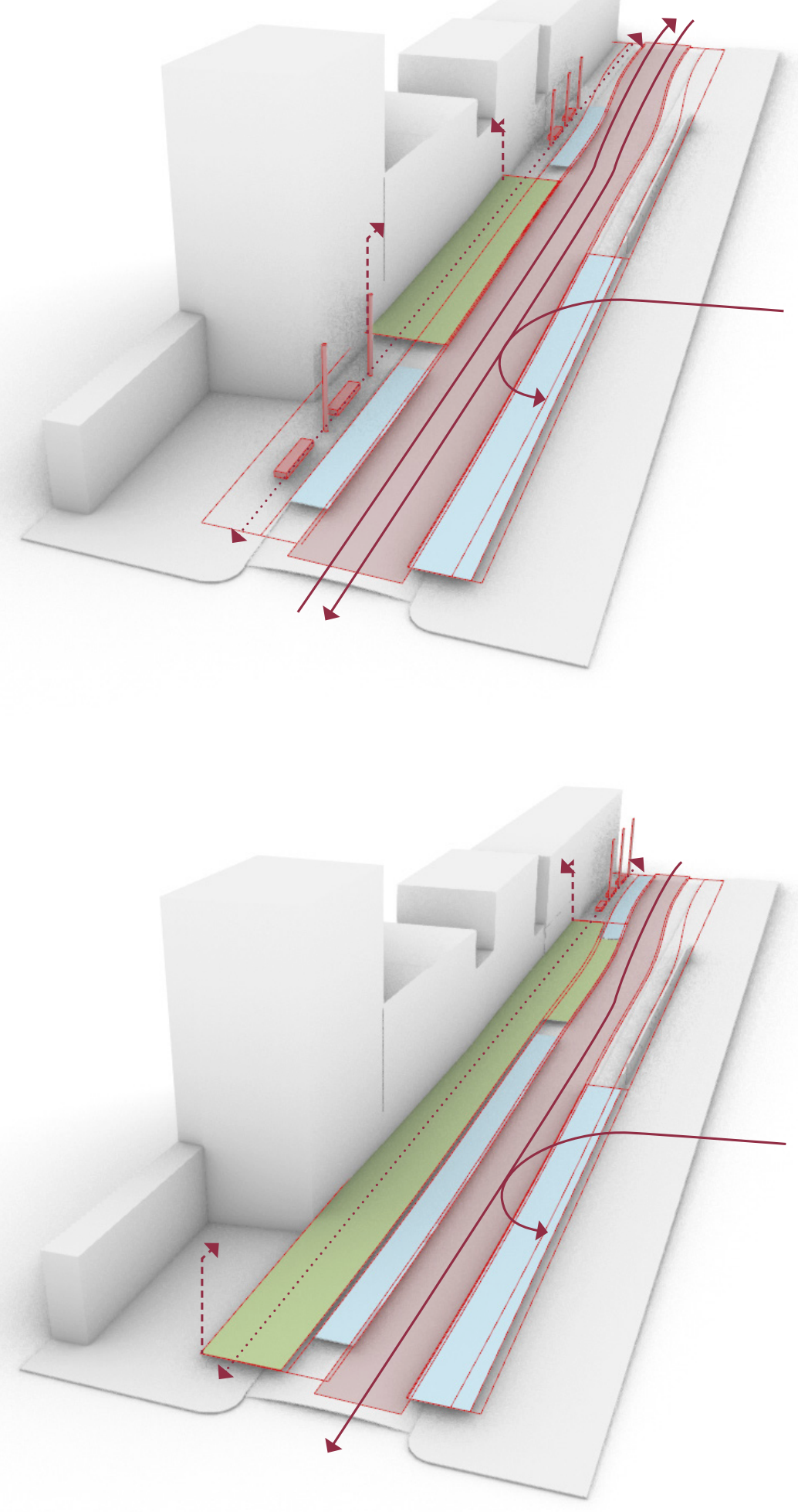

Figure.1.26. Tory street, vehicle access.

Study $1 \mid$ Page 53 


\section{Study 1 - Conclusion}

The structural defining characteristics of the principal, the logic and the parameter helped to think through and plan strategies for developing the script and enabling geometry relationship structuring. What was especially complimentary to the process was the use of sketching and diagrams. The diagram was vital in logic planning, using few coloured pens to either represent curves, points and then also the function/ desired action, usually represented with arrows suggesting displacement of an element.

While it was apparent that the script followed a linear and sequential process, this may be the result of three things. Firstly the linearity of the street and the simplicity of the rectangular parameter, confined by the building façades. Secondly, the approach to the exploration of scale. The process followed a

scale-down method where low logics naturally responded to a larger scaled element, ordering from region to parking to street side element. Thirdly, the simplicity of the script itself, while this was important to establish the basic principles of parametric thinking, the lack of complexity may have compromised the conclusiveness of the relationship structure and this type of flow structure may not be the case under different utilisation of the tool. Going forward, the idea of the logic, the principal and the parameter will be re-worked in terms of how they coordinate themselves within alternative design scenarios.

Responsive programming implies a series of IF $\mathrm{x}=\mathrm{y}$ statements, for example if the kerb moved $4 \mathrm{~m}$ from the site boundary then parking is allowed to occur, IF ("distance" $=4 \mathrm{~m}$, true, false).

This type of truth statement is an inherent part of parameter thinking, in computer language, true, false, 0 or 1 allows the computer to understand if a threshold has been breached. In a tool sense, this then allows for a greater control of data flow.

The 'stream filter' component in Grasshopper is a prime example of this function (Fig.9). Different data sets might be plugged into various input gates of the component, and a truth statement might be plugged into the input of the gate control $(G)$, depending on the input statement, output 0 or 1 will activate and the correct flow of data will go through. This in fact, was how the dynamic region logic was programmed. If false was given, the point of most depth occurred on the left and if true; the right. If false, the program region will generate on the left, if true, the right.

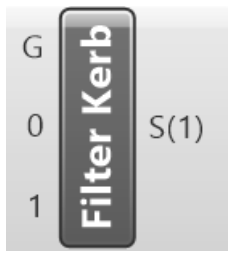

Figure.1.29

Stream filter component

from Grasshopper. 
To summarise the key points of this study.

- $\quad$ Linearity of site and clear real world boundaries, allowed for simple representation, application and manipulation of constructed parameters in Grasshopper.

- $\quad$ Hierarchy is important to the flow-on effect of shifting parameters. A clear hierarchy of logics helps to manage the correct flow of data and ensure the appropriate geometric response to a given.

- $\quad$ The diagram is an invaluable tool to parametric thinking. Logics can be well thought and tested through sketch, thinking on paper the relationship between points and curves.

- $\quad$ Thinking through the paradigm of algorithmic tools can be structured by distilling the process to the ordering of principals, logics and parameters.

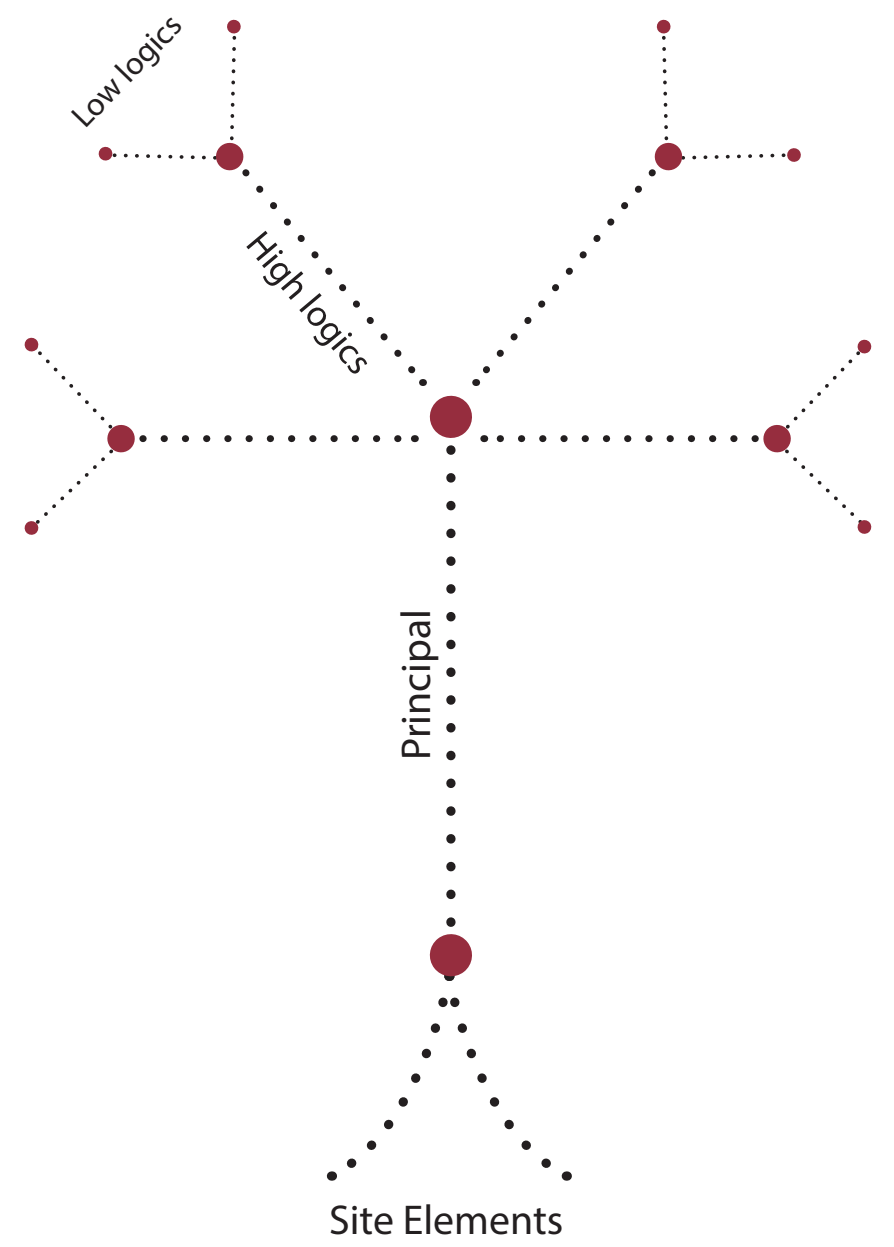

Figure.1.30. Diagram of script structure, study 1. 


\section{Study 2 - Introduction}

Responding to the linear rigidity of the first study, set within a streetscape context, the second study is set in stark contrast, engaging with a coastal montane site. Moa point on the south side of Mirimar Peninsula, Wellington, New Zealand, provides much more topographic variation and dimension as a testing ground for both topographic and ecological parametric engagement.

The study unveiled an extensive level of utility regarding the mesh geometry, both as a representation of topography but also as a catalyst for simulation. By identifying the relationship between slope and vegetation within one of the valley systems on site, these patterns could be replicated using point geometries projected onto a mesh. The dynamic nature of Grasshopper geometries meant that the mesh as a facilitator for projected inputs could be manipulated, pushed and pulled to give metric feedback for changes to the vegetation pattern.

To engage with the simulative mesh geometry, the study utilised the toolmaking qualities of Grasshopper. Developing a series of tools to construct a pathway through the valley system, operating directly with the mesh, testing design potential under the guidance of metric feedback. However, paying particular attention to the creative engagement of operating the developed tools.

Finding it immensely more difficult to apply strict parameters to Moa Point opposed to Tory Street, the applicability of parametric toolmaking became quickly apparent for its flexibility and problem solving.

\section{Study Two, Aims and Objectives}

- Understand responsive parametric operations in a coastal montane site.

- $\quad$ Extract thought pattern/process pertaining to algorithmic rules and restrictions.

- $\quad$ Build upon, and challenge the ideas and perceptions from the first study.

- $\quad$ Review the hindrance of creativity under the investigated methods. 


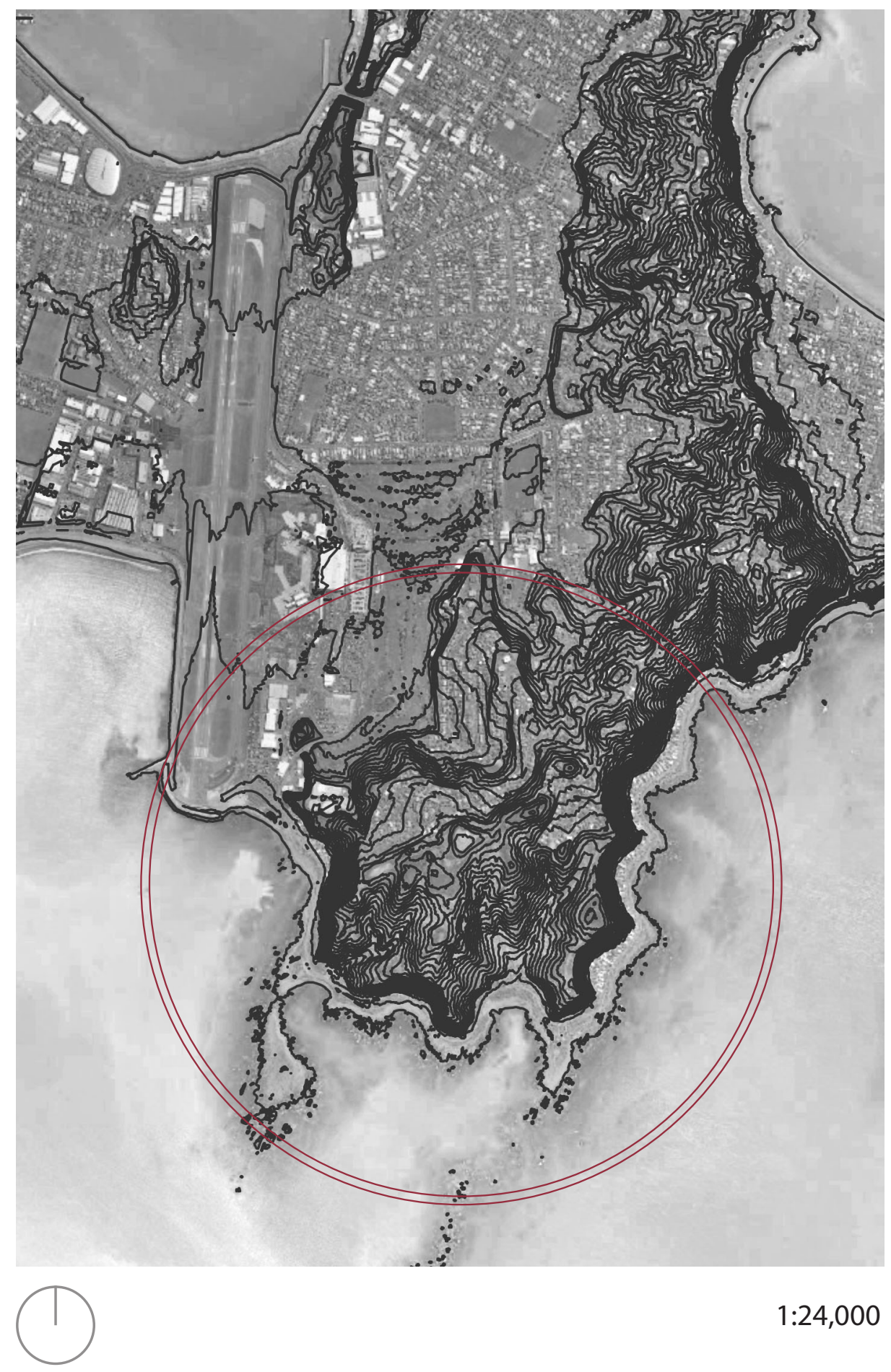

Figure.2.01. Aerial of Moa Point. 


\section{Microclimates}

Patterns in vegetation were clear on site. Complex at a large scale, site conditions such as wind exposure, moisture and slope, contribute to the distribution of plant species. Identifying the microclimates through isolating conditions to specific quadrants of site, correlation between plant type and slope becomes easier to trace as conditions such as wind and sun exposure becomes fairly unified.

By figuring out the slopes that core plant species occur at, these species can then be represented by points on a digital mesh. With this information, changes to the mesh can respond with changes to vegetation distribution for use in assessing how a design might affect existing vegetation on site or for use in planting strategies considering plants with similar characteristics to the existing vegetation.

This study looked at one of the valley systems exploring this concept. Considering the valley system, vegetation was identified on site and assigned groups relating to specific slope domains, slopes at $0 \%$ to $10 \%$ with a lower elevation and concave areas for example would group valley floor vegetation in which tends to be denser.

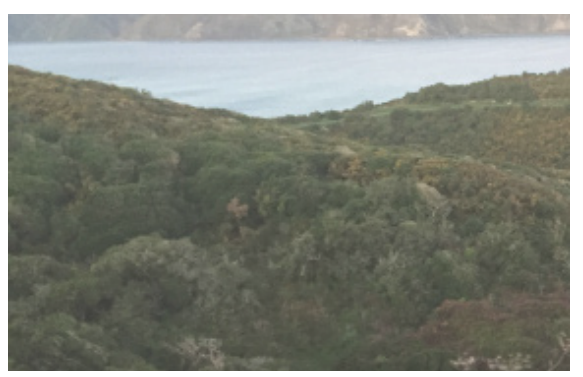

Figure. 2.02. Author's photo of neighbouring ridge. Gorse can be seen on the peaks of the ridgeline, a gradient of vegetation density can be seen creeping towards the valley floor.

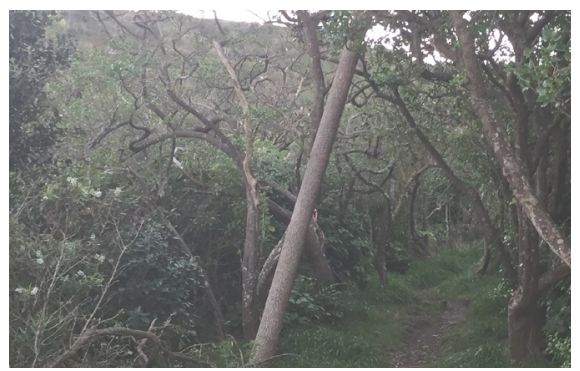

Figure 2.03. Author's photo from the valley walking track. Kohekohe trees can be seen arching over the pathway, high density of vegetation on either side.

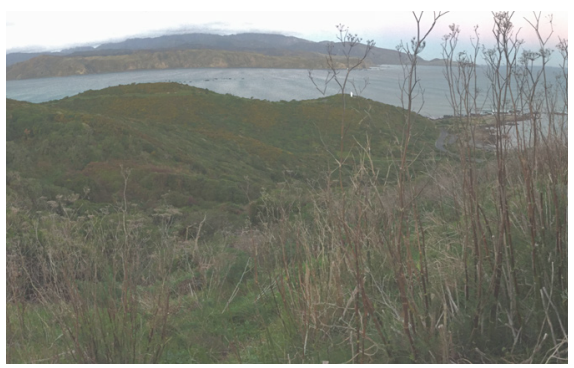

Figure 2.04. Author's Photo from ridge line. Fennel growing on the steep slope in the foreground. 


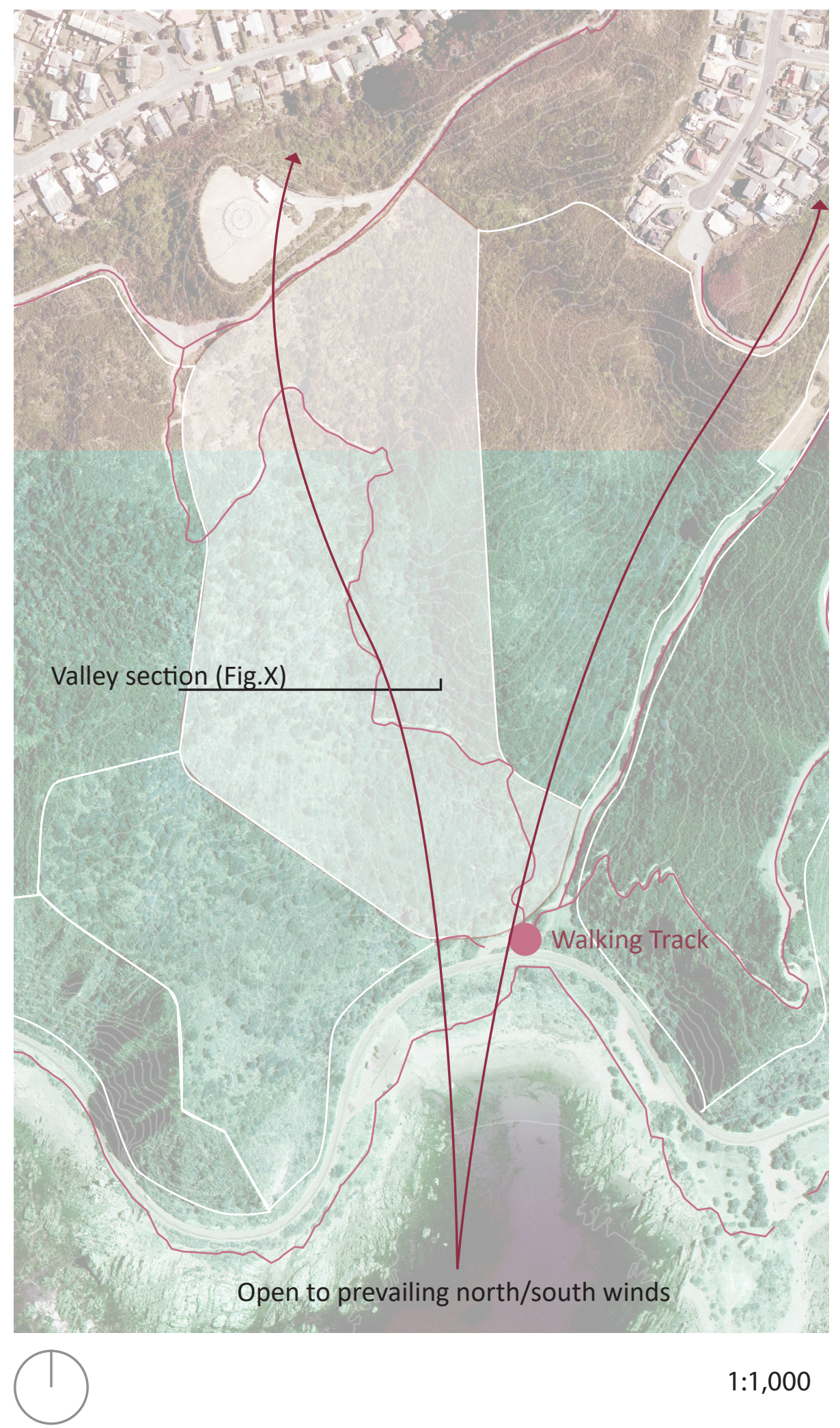

Figure 2.05. Map of Moa Point, fragmented into regions roughly depicting regions as separators of condition and microclimate. 
The site is dominated by dense Kohekohe groves, the valley undergrowth habituating Kawakawa, karo, Toetoe, Shinning spleenwort fern with few open patches of bracken fern, Cordylines also breaking the canopy in some areas. The ridges and higher elevated areas have breakouts of gorse, as well as fennel being apparent on steeper slopes and ridge spaces where gorse hasn't occupied.

\section{Fennel, Flax, low growing}

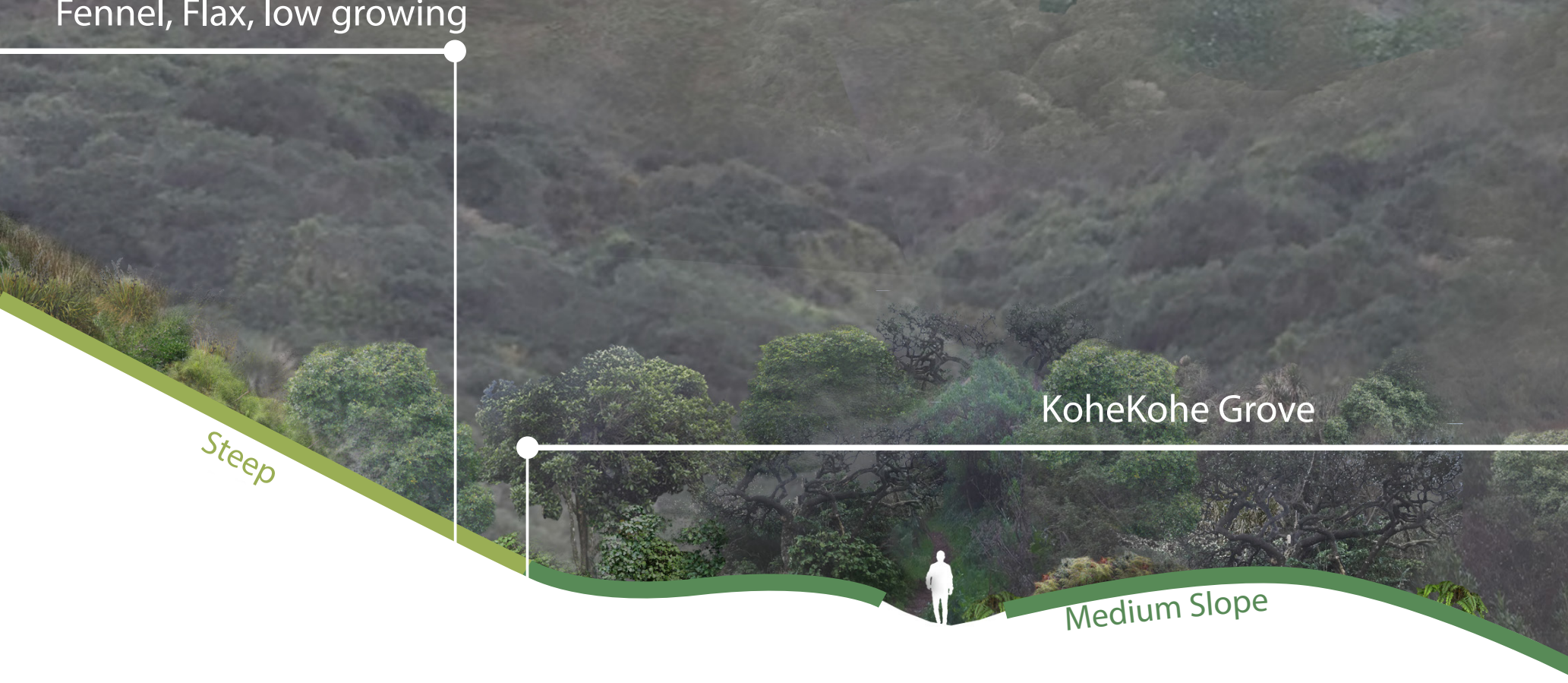

Figure.2.06. Valley section of Moa Point.

Page 60 Study 2 
Gorse ridgeline

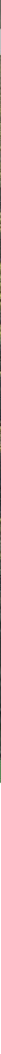




\section{Existing Vegetation Sample}

The plant species were split into 4 slope domain groups. Blue, concave and low flat elevations between $0 \%$ and $10 \%$ slope. Dark green, medium slopes, between $11 \%$ and 25\%. Light green, steep slopes, \%26 to \%100. Lastly, yellow Concave and high flat elevations between $0 \%$ and $10 \%$.

\section{Exposed and steep Slope Vegetation}
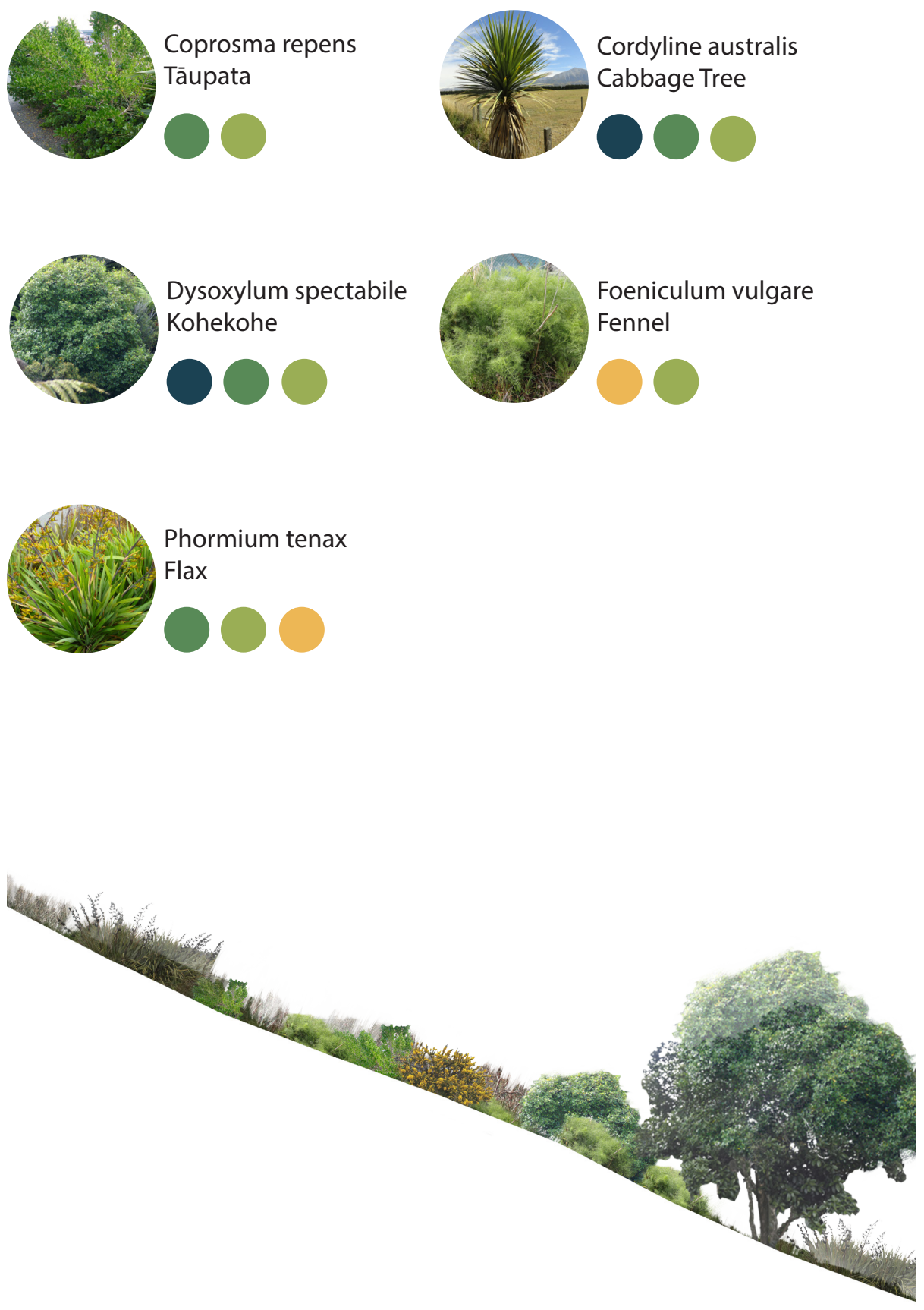

Figure.2.07.1. Exposed slope vegetation palette. 


\section{Valley Vegetation}
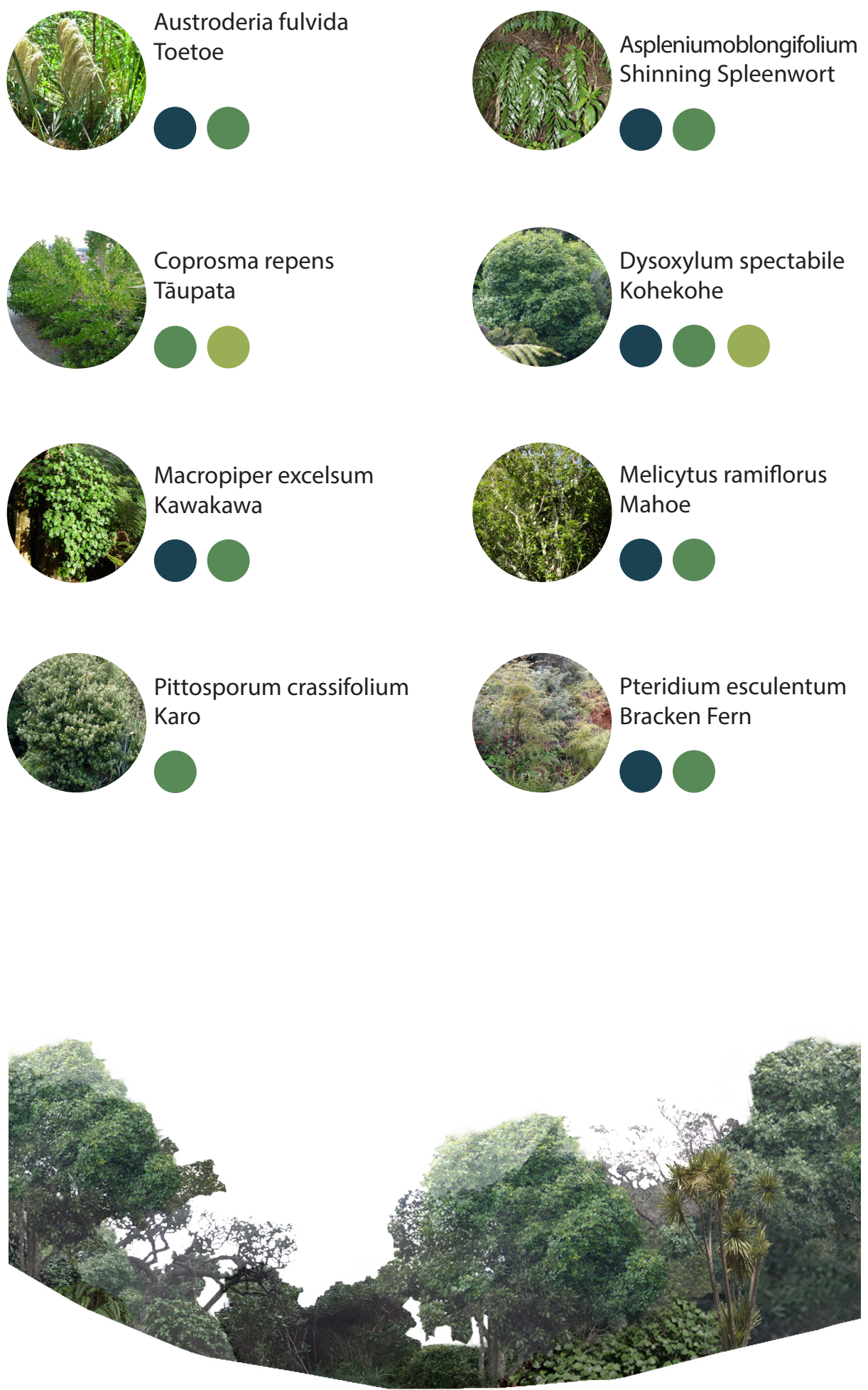

Figure.2.07.2. Valley vegetation palette. 


\section{Ridge Vegetation}
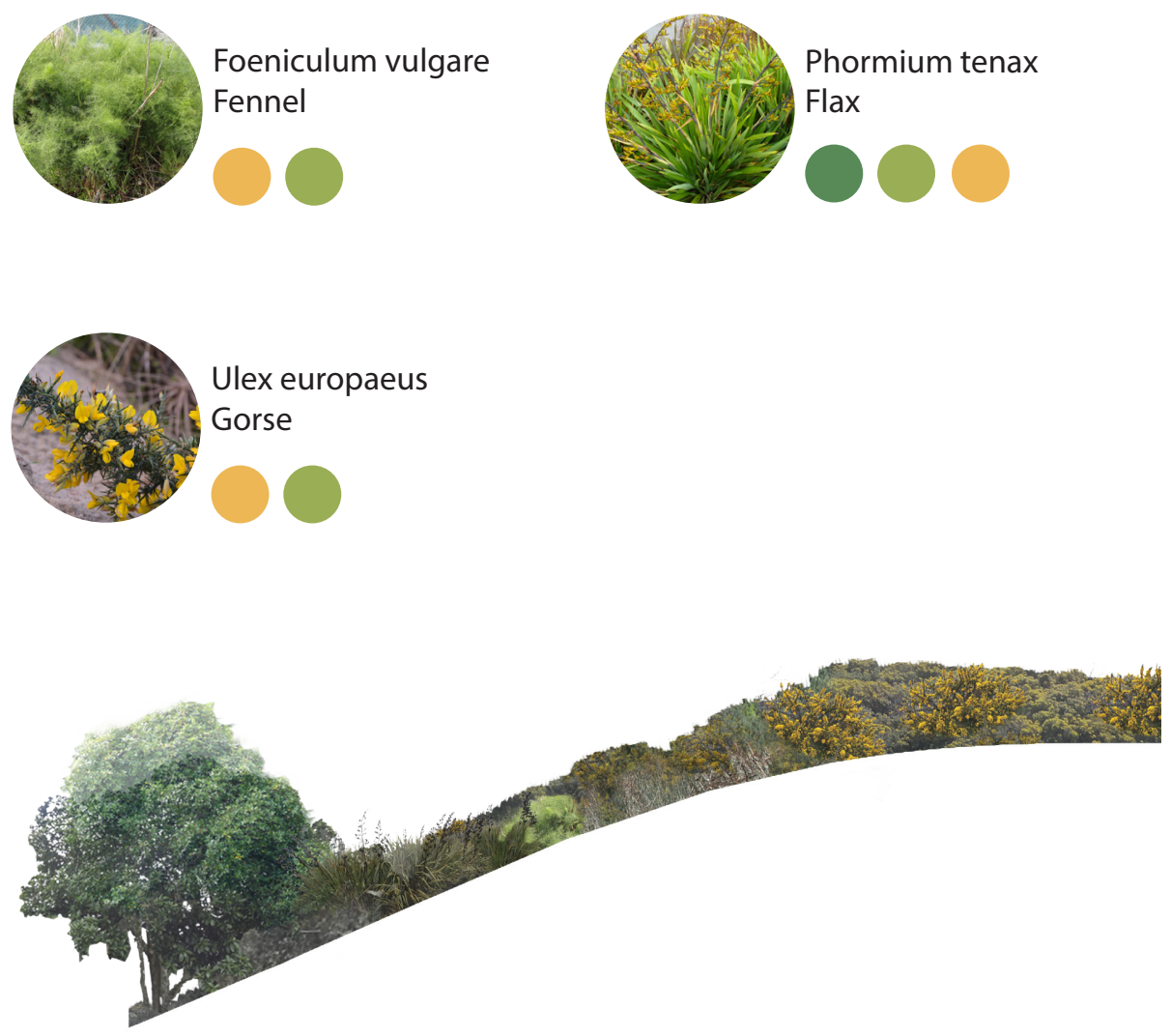

Concave areas usually occurring in the depths of the valley, consisted of the least drained soil, hosting the most variety of species. While Concave ridgelines and peaks were commonly attacked by gorse due to harsher conditions. 


\section{Pattern and Simulation}

Through identifying the correlation between slope and vegetation, slope domains can be applied to a digital mesh to extract the faces (or polygons) as per their Pythagorean properties.

"Patterns are synonymous with processes; they are indications of the forces and interactions that created them." (M'closkey \& VanDerSys, 2017)

Patterns are prevalent in life, they are key to a functioning system. Pattern by definition notes an evolution, or symmetrical fractal effect. Patterns exist at the core of genetics, most noticeable in the growth of plants where a species has its own recipe for form. Whether it's the alternation of leaf structure or the spiralling pattern of a hurricane patterns occur at all scale.

Further noted by Karen M'Closkey and Keith VanDerSys.

"Humans have an innate ability to recognize patterns; our brains are wired to perceive them and to seek them if they are not immediately visible. We look for patterns in nature in order to understand relationships between function and form, as in morphology, and between information and communication, as in genetics." (M'closkey \& VanDerSys, 2017)

The utility of understanding patterns is only amplified by an algorithmic toolset. Through geometric relationships, patterns can be represented dynamically as opposed to analogue methods of mapping where patterns are statically represented, usually over flat planes.

Algorithmic pattern representation allows the designer to interpret responses based on their knowledge of site and processes. It allows for simulative toolmaking and conceptual development derived from the probing of certain systems that exist on site. However this type of toolmaking is restricted by the complexity of the problem, for instance, this study was required to isolate one of the valley systems rather than consider the whole site due to variations of micro climate and CPU requirements of Grasshopper. By representing the vegetation through slope groups, this study if anything was a personalisation of interface, based on the assumption that; "by the vegetation pattern existing on site, these are the slope domains that parametrise these specific types of plants. Therefore, if terraforming is to happen on site, then this (the response) is the likely impact of vegetation distribution, assuming a natural state." 
Slope alone was not enough to represent the vegetation on site. A split at mid elevation had to be introduced to differentiate the flat areas of the valley and the ridge lines. Concave and Convex areas were also extracted for the same reason.

\section{Slope Correlation}

The site was divided into four slope categories as seen below. Overlapped with a baseplan correlation between planting pattern and slope can be seen.

High Gentle, Convex

Steep
Medium

Low Gentle, Concave

$\% 0$ to $\% 10$ occurring at high elevation.

$\% 0$ to $\% 10$ occurring at low elevation.

Figure.2.08. Mesh and slope. 


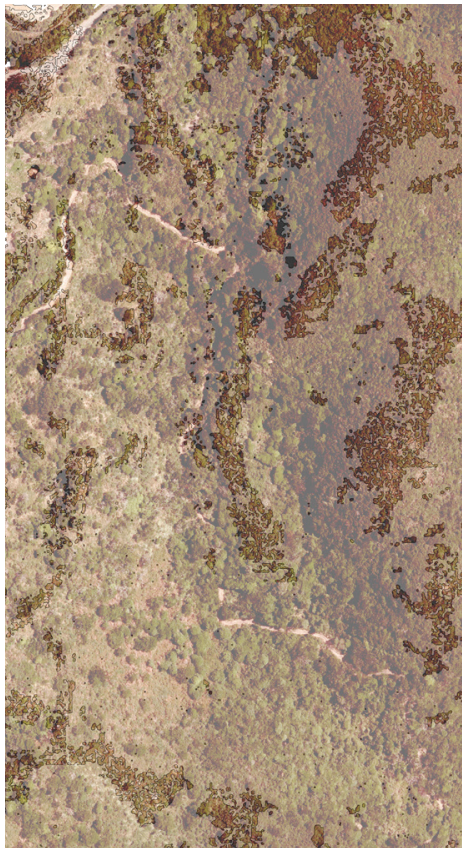

Convex \& High Elevation Gentle $\% 0$ to $\% 10$

Figure.2.09. A correlation between low growing shrubs and convexity was apparent. Convexity can be used to determine well drained areas of the site.

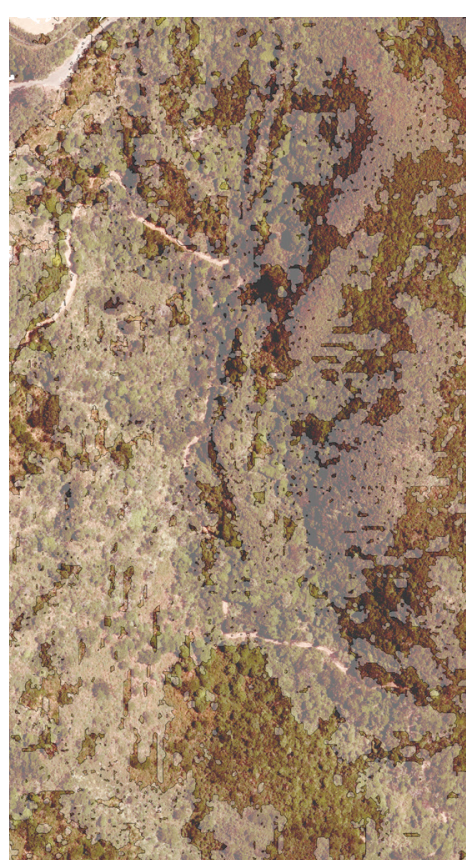

Medium \%10 to \%25

Figure.2.11. Medium slope gradients tend to grow a diverse range of species. Soil permeation levels being the most sensitive of the slope types.

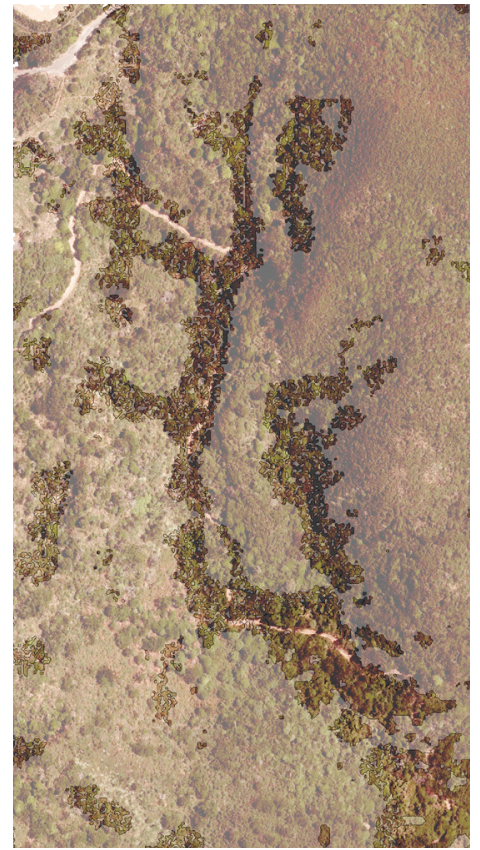

\section{Concave \& Low Elevation} Gentle $\% 0$ to $\% 10$

Figure.2.10. Concavity can be used to determine lesser drained areas of the site, isolating dense valley vegetation.

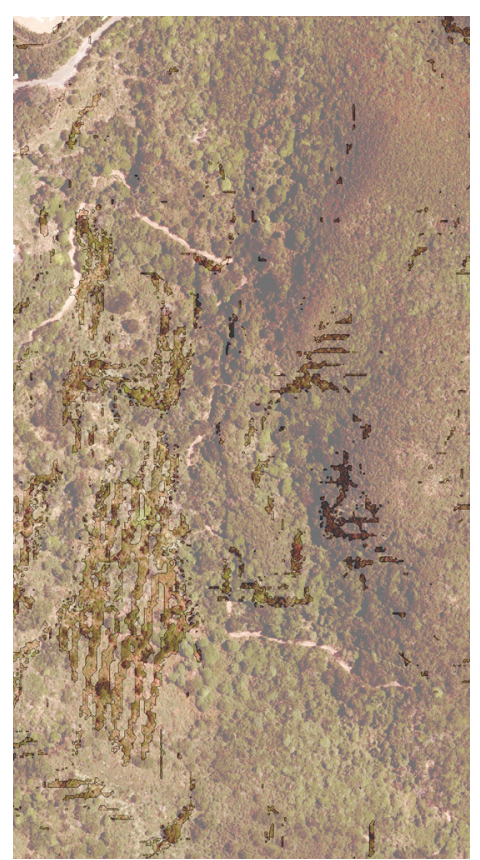

\section{Steep $\% 25$ to $\% 100$}

Figure.2.12. Steep slope gradients correlate with hardy, low growing shrubs, bare rock and cliff faces. 


\section{Points and Density}

The next step in building the model was through representation of density. Points were projected onto each slope group, restricting the amount of points being generated on well drained areas, peaks and ridgelines (convex, $\% 0$ to $\% 10$ at high elevation and steep slopes). Also adding more points to areas of poorly drained soil, primarily the valley (Concave, $\% 0$ to $\% 10$ at low elevation). Medium slopes had slightly less than concave and low flat slopes.

The points could then be measured metrically, with the original mesh consisting of \%15.7 concave and high flat vegetation growth, \%12.5 steep vegetation growth, \%50.3 medium vegetation growth and \%21.6 dense valley growth. As these points are in a dynamic relationship with the mesh, changes in the topography will provide changes to these numbers. This, giving example to the mesh operating as catalyst or centralising geometry of its own information.

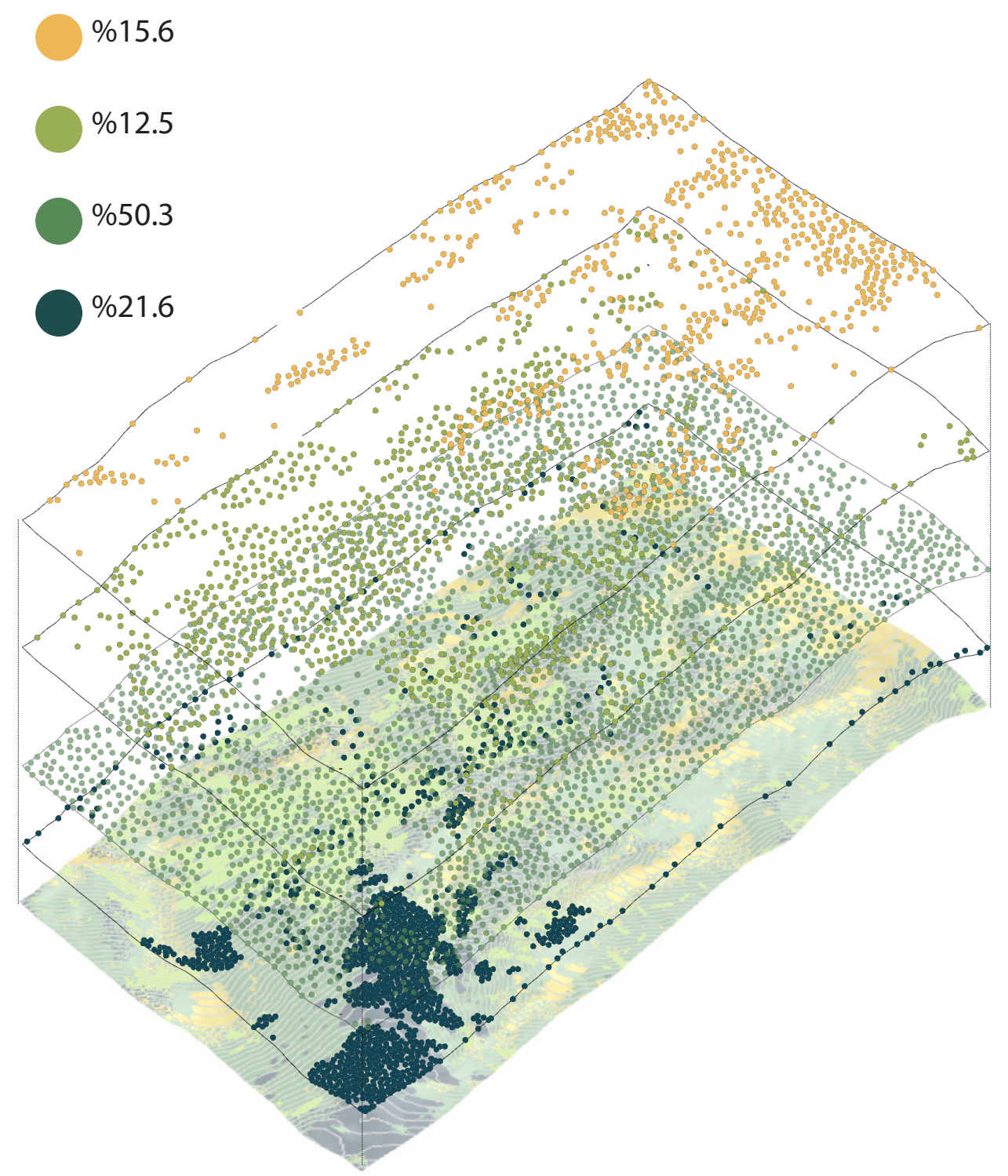




\section{Mesh Manipulation}

Of parametric geometries, the topographic mesh seems to have most significant adoption of landscape qualities for its representation of topographic sites.

A mesh is made through the welding of polygons, which by increasing the polygon count, the quality of the mesh is also increased. The properties of the polygon provides means of manipulating the mesh through the evaluation of its edges, center points, area, slope and shape.

With the appropriation of sensory technology such as LIDAR, we can produce high quality meshes. Enabling dynamic algorithmic engagement with accurate landform representation.

What this means is that landscape architects can simultaneously be terraforming while receiving feedback pertaining to topographic mesh properties, for example; cut and fill, contour regrading, watershed, drainage data and slope information, but also measure of linked geometries such as the points used to represent vegetation in this study. Providing efficiency to technical outputs and feedback-loops for conceptual testing.

The Grasshopper plugin 'Bison' was immensely useful for its ability to manipulate and analyse the mesh through assigning pull/push functions to points and curves and providing analytical tools for assessing watershed, runoff, slope and concavity. Simplifying the interface between point, curve and mesh, reducing the size and CPU requirements of the Grasshopper script. Also re-enabling a familiar syntax of points and curves. As Grasshopper simplified the syntax of Python, Bison and other plugins alike simplify the scripting interface further with custom components, built for specific tasks. Conventionally landscape architects shift topography by regrading contours in CAD, a tedious task. With an accurate mesh geometry contours can be created and worked with in Grasshopper, and Bison allows the contours to be worked with fairly simply as is seen in the accompanying images. Although CPU limits the mesh detail, meaning the contours generated would not be engineering quality. At this stage limiting the tool to conceptual testing. (Vashakmadze, 2018) 


\section{Content unavailable}

\section{Please consult print version for}

\section{access.}

Figure.2.14. Conrad Waddington's (1957) conceptualization of an epigenetic landscape (Kwinter \& Boccioni, 1992, p. 62)

"The guy-ropes are tethered not only to random points on the overhead surface but to points on other guy-ropes as well, and to pegs in the lower surface that themselves represent only semi stabilized forms, thus multiplying exponentially the non-linearities flowing through the system." - "No change in any single parameter can fail to be relayed throughout the system and to affect, in turn, conditions all across the surface." (Kwinter \& Boccioni, 1992)

A geometric concept invented by Conrad Waddington in the 50's. This is still relevant in the conceptualisation of mesh geoemetry.

\section{Bison Toolset}

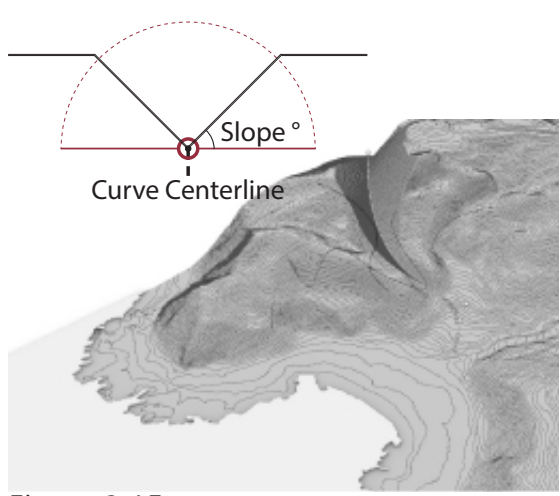

Figure.2.15.

Mesh Curve, applies fitting function to curve with a specified slope

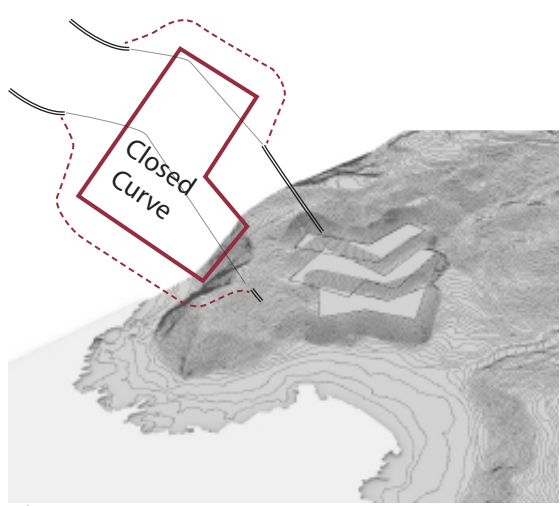

Figure.2.16

Mesh Flat, applies fitting function to a closed curve with specified edge buffer.

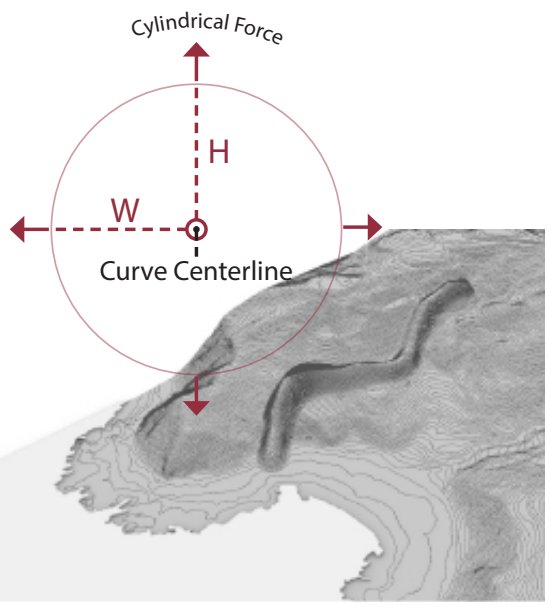

Figure.2.18.

Mesh curve pull, applies cylindrical force to a curve.

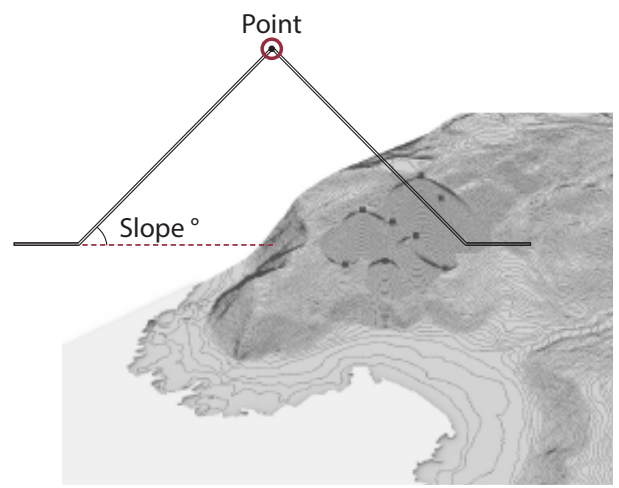

Figure.2.17.

Mesh Point, applies fitting function to point with a specified slope 


\section{Toolmaking}

Cantrell and Meikies note that the toolmaking process has been enabled through various recent developments such as open source coding, hardware production and cloud connectivity. Allowing for personalisation and augmentation of existing software as well as easy to transfer file types between programmes, meaning design can be transferred between software with specialised toolsets, such as from Arcmap to AutoCAD to Rhinoceros to Sketchup. (Cantrell \& Mekies, 2018)

Grasshopper's dynamic and algorithmic structure enables toolmaking at a high level. The visual coding aspect of Grasshopper has made coding tools much more engaging, and as designers are inherently "visual", it has made algorithmic thinking much more accessible in fields of architecture and design. (Wallis \& Rahman, 2016)

The art of toolmaking opens up new paths of design generation for landscape architects. Parameter thinking inherently gets you to think in terms of boundary, it encourages you to seek them. In this study, the first tool created took a simulative and metric approach, isolating slope types on the mesh and generating point densities in accordance with existing vegetation distribution. This allowed for a new way of engaging and testing site in order to extract concepts. Very much opposed to the first study however both seeking the same goal.

With the simulative mesh created, the next step was to challenge it with design. To do this, a goal of creating a path from the bottom of the valley to the top was given. The path needed to interact with the mesh, triggering change to the points. For this, easy control over the path, maintaining a pull/push interface was crucial in promoting and exploratory interface. The creation of the path tool had two primary functions, regrade and fit the path to the mesh and generate switchbacks where the curve kinks in order to traverse steep slopes quickly. The width of the path could also be controlled on a slider with a minimum of 1.5 meters. For the script to run, the only input needed was a single curve that traversed a desired path.

For regrading the mesh, the Bison plugin's "mesh curve" tool was used, this fitted the path's edges to the

mesh. The inner path was used to trim the mesh, leaving a void, this was to stop points from spawning inside the path so that metric data was accurate. 
Further tools were created to analyse and support the creation of the path. The curvature of the path could be assessed for both slope (Fig.2.20) and variation (Fig.2.21). Lastly a tool was created to assess immediate points that the path traversed through. A grouping mechanism was created that collected points within certain range of the pathway. With this tool, the path could be assessed for the type of immediate vegetation character at any given point along the journey. Isolating these points gives suggestion to the type of experience the journey offers. The immediacy of this, allows for tweaking and experimenting. (Fig.2.22)

\section{Path Tool}

Applies switchbacks to the path at kinks in the curve.

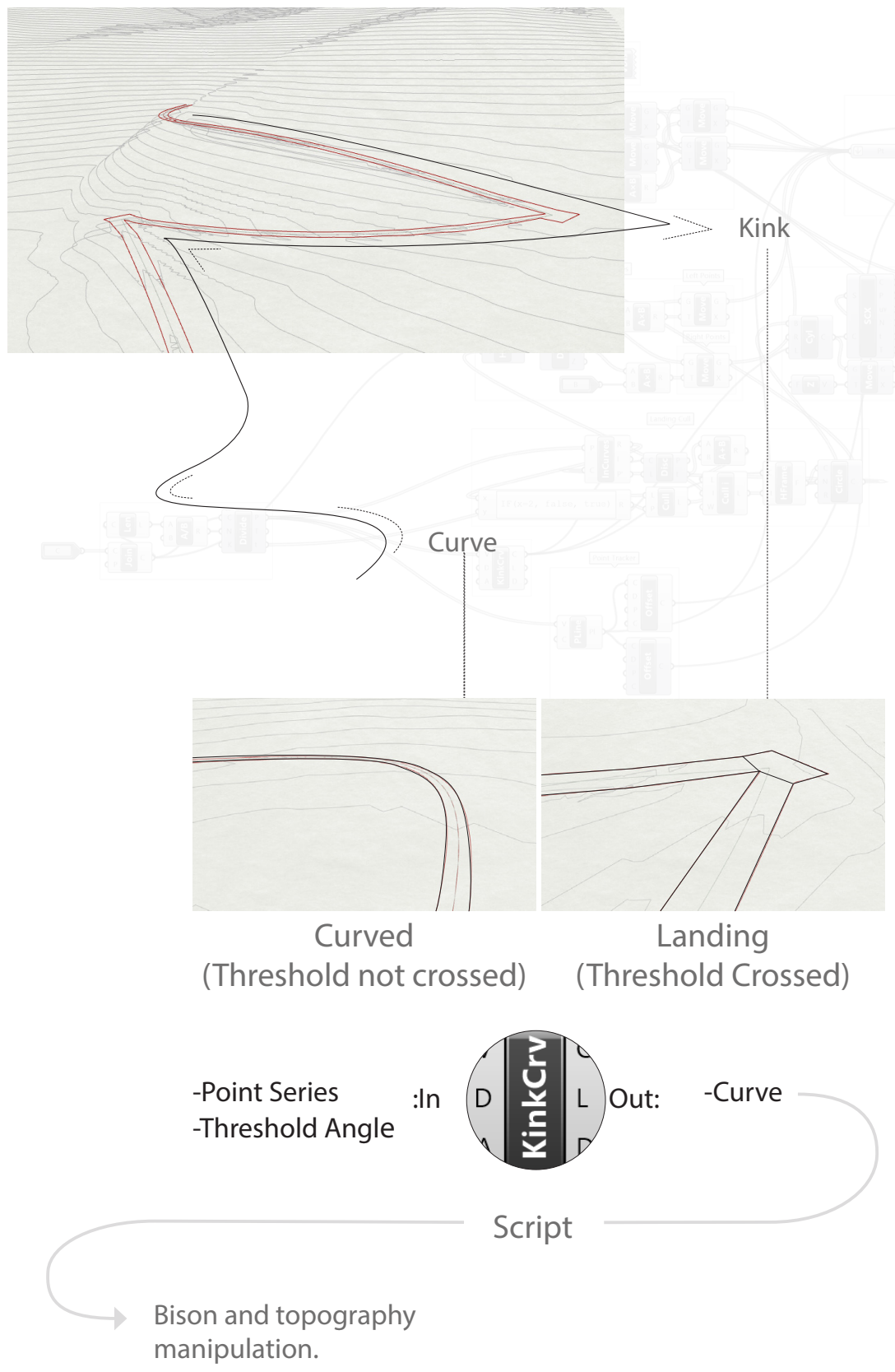

Figure.2.19. Path tool for Moa Point. 


\section{Path Evaluation Tools}

Further evaluation tools were produced to understand the character of the path and the journey.

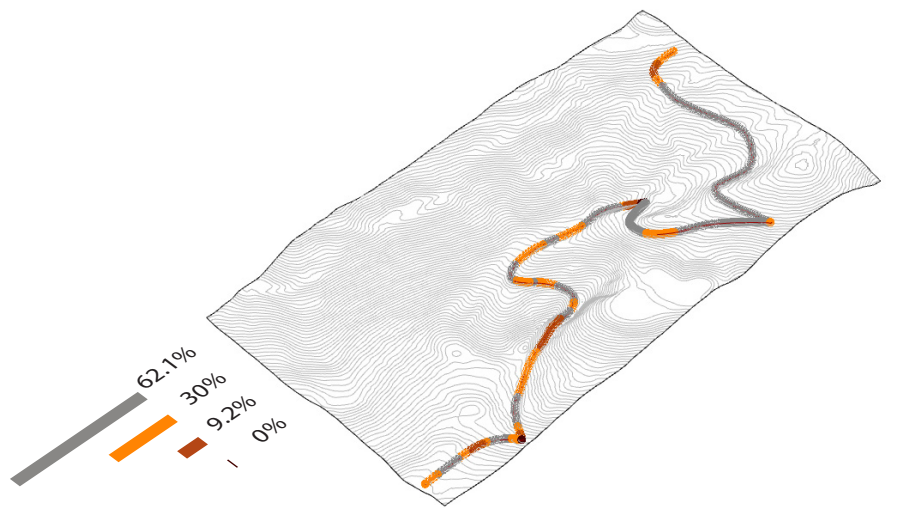

Figure.2.20

Slope analysis of the pathway. Colours were applied to different slope domains. 0\% to $12 \%$ for example was coloured grey. If coloured red, the slope is \%40 and over which may require change to a more appropriate slope level.

Figure.2.21

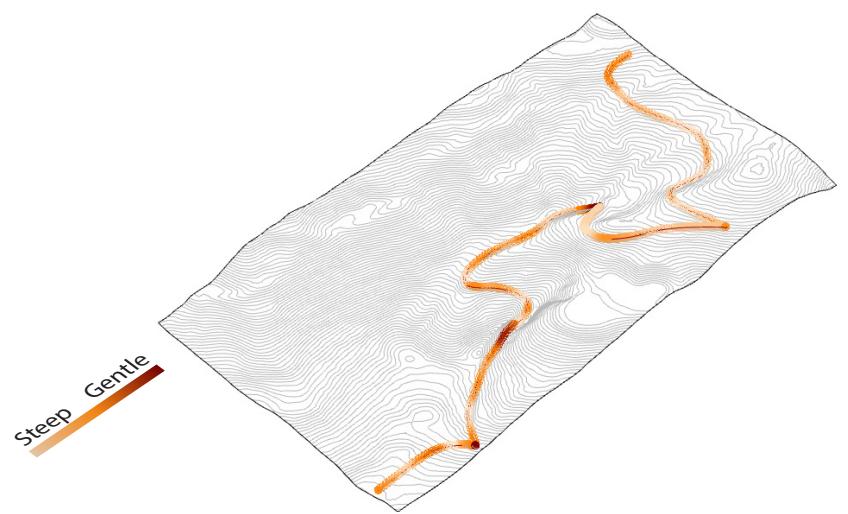

Path immediate vegetation. Isolating the immediate points giving a sense vegetation type that the path is interacting with. The pull/push nature of the path manipulation allows for rule setting to explore potential options. For instance, setting a minimum for plant type that the path must traverse through.

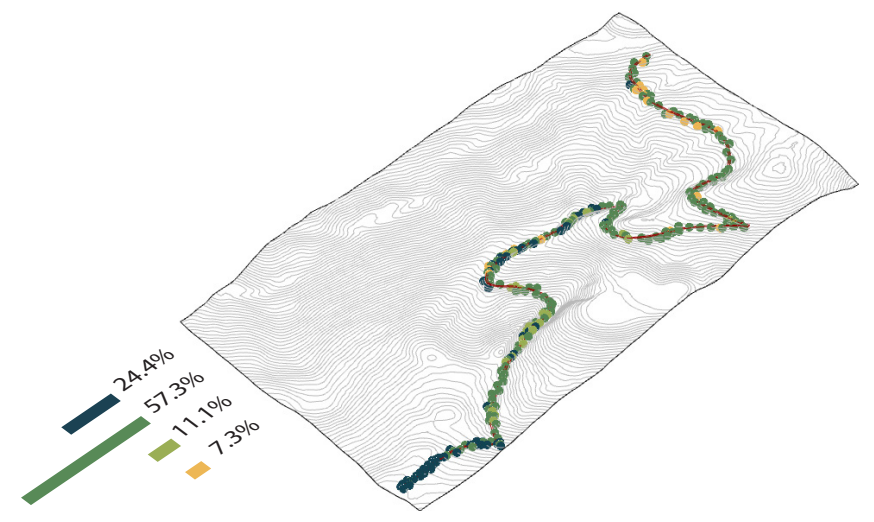

Figure.2.22

Path Slope Variation. Assessing the slope of the path however assessing the variation along the entire pathway assessing difficulty and character. 


\section{Feedback loops}

Ludwig Von Bertalanffy's General Systems Theory describes open systems as a cross relational and scaling inter-communication between systems. In an ecological sense it implies that there are no boundaries between ecosystems, that there is a constant flow of energy exchange, of inputs and outputs. (Walonick, 1993)

"There are no separate systems. The world is a continuum. Where to draw a boundary around a system depends on the purpose of the discussion." (Meadows, 2008)

Engagement with open systems has always been an elusive and difficult task of landscape architects. Specialists are often required to fill the gaps of knowledge that designer's haven't the time to understand. Landscape architects have the acute ability to link design form to the needs of site and context, thus communication amongst disciplines is vital for good urban development, BIM software being built around this principle is exciting for this reason. The conceptualisation of feedback loops however offers a new way of design consultation, one that is automatic and immediate. It offers new methods for conceptual development.

"In this dance between "generate" and "test" there is a continuous feedback loop of iteration and refinement, ultimately leading to some "final" design decision, based on a heuristic approach, not specified by any algorithm." (Cantrell \& Mekies, 2018)

While it seems contradicting to suggest communication between disciplines is vital; then to further suggest a computer can offer means of the same consultation. I would argue a point of interface, that testing form against natural systems, receiving feedback, refining the design, then testing and so on - may lead to more refined and sensitive outcomes, with the designer making subtle and gradual design shifts based on the feedback received. The feedback loop then can be thought of as an added sense to the designer amongst the visual and the intuitive. Under this concept this also means that when specialist consultation is required, the design has already been through an automated primarily analysis. 


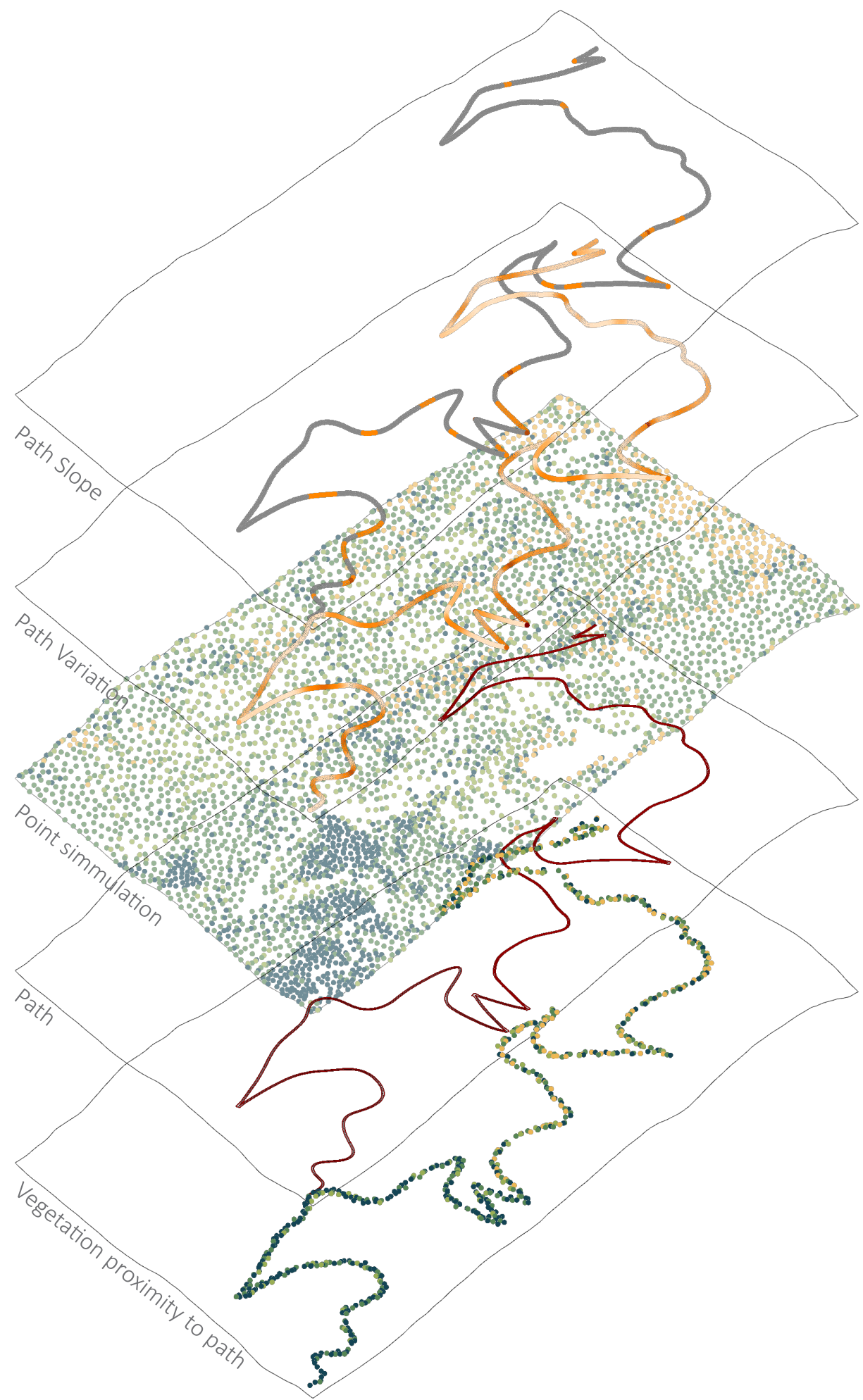

Figure.2.23. Feedback loop model representation for Moa Point. 


\section{Augmented Workspace}

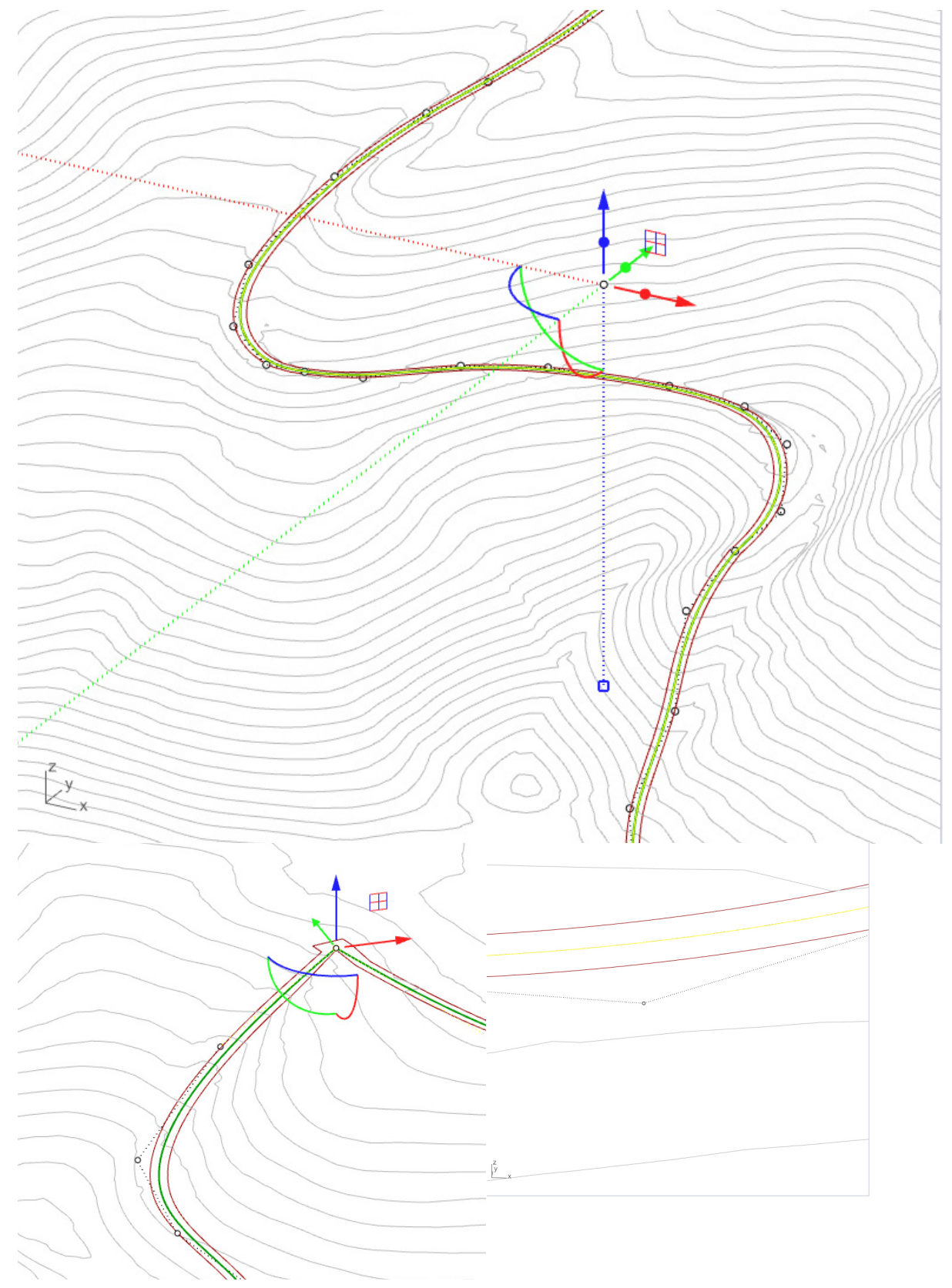

Figure.2.24

Using a single curve, the script applies switchbacks at kinks and regrades the contours by fitting the mesh to the path. The control points of the curve geometry allows for pull and push modality. 


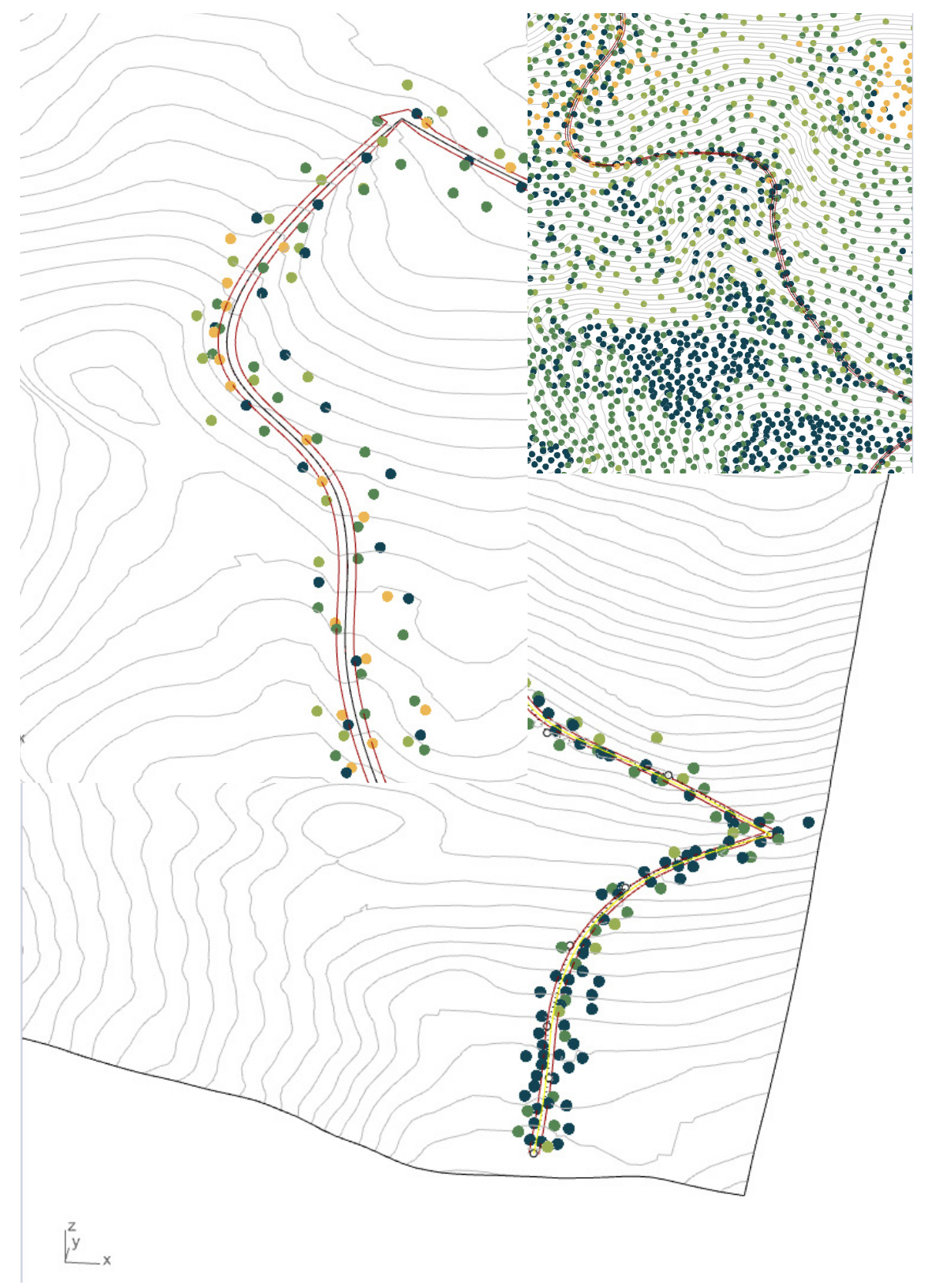

Figure. 2.25

Displaying the point density and how the path is interacting with the points and mesh. 


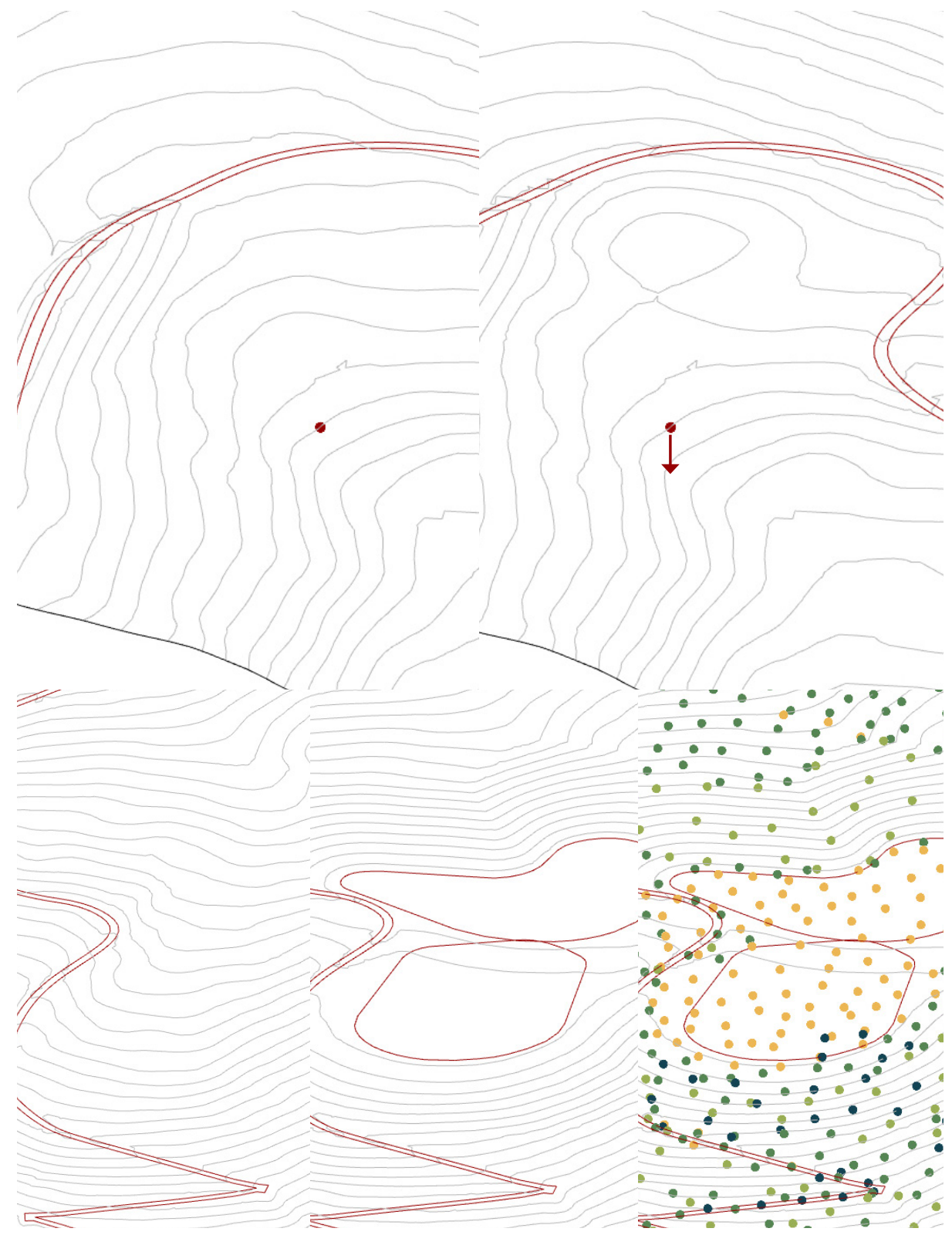

Figure.2.26.

Displaying other Bison components, the 'mesh flat' component creating terracing in the bottom images and 'point-pull' creating a retention basin in the above. 


\section{Variation 1}

The three following variations diagram the engagement with tool. Producing three different path options with varying metric feedback.

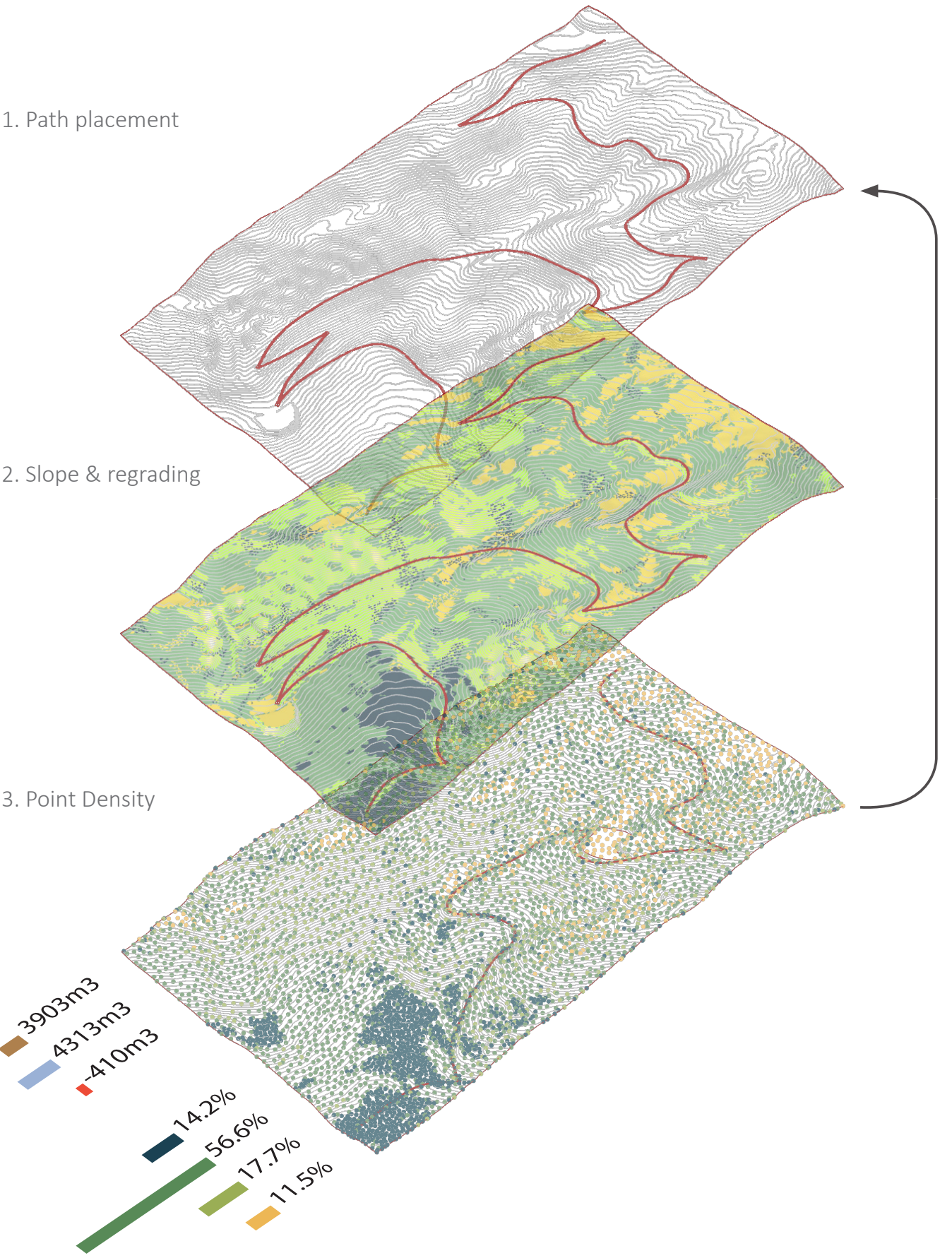

4. Metric feedback

Figure.2.27 Path test, variation 1. 


\section{Variation 2}

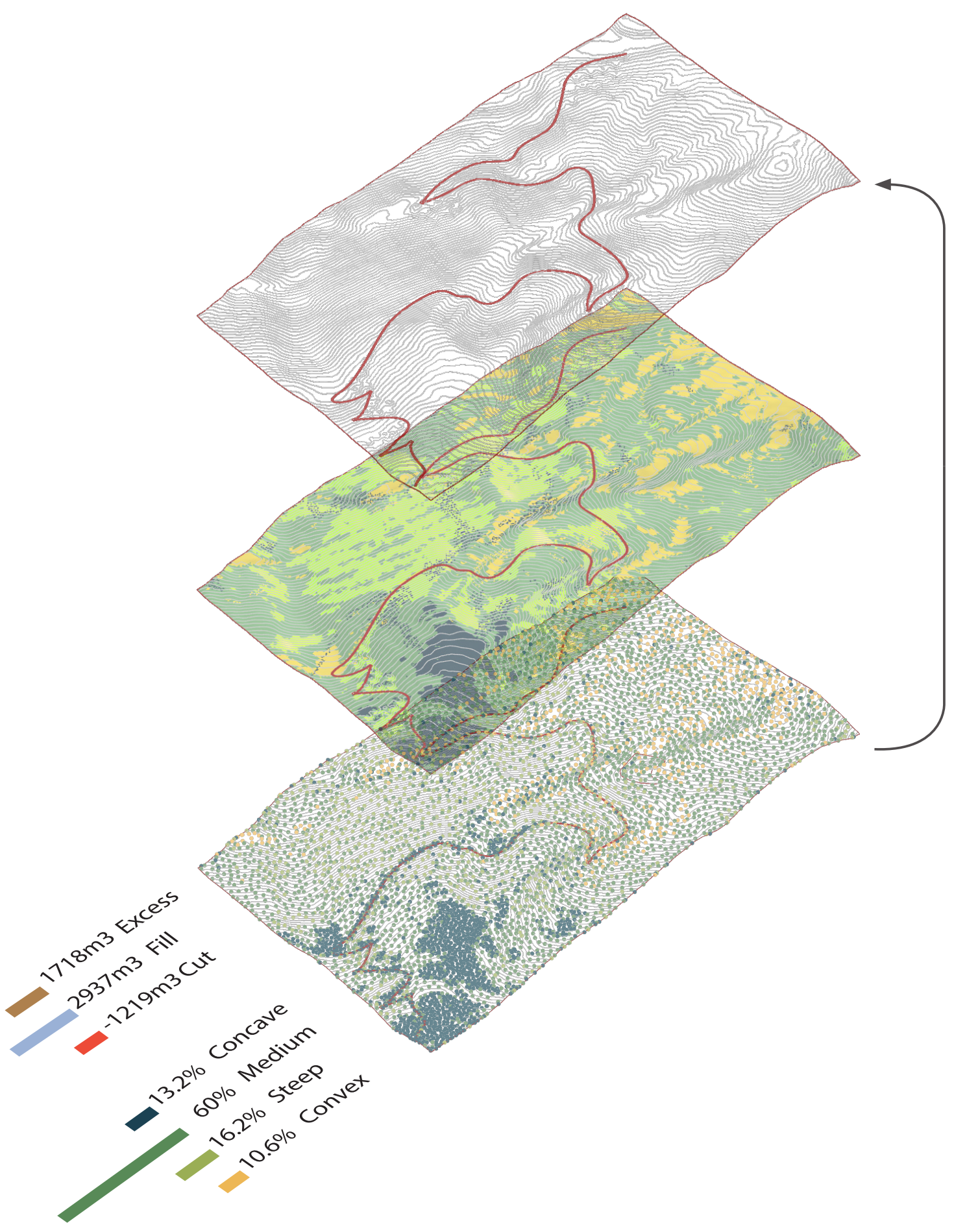

Figure.2.28. Path test, variation 2.

Page $80 \mid$ Study 2 


\section{Variation 3}

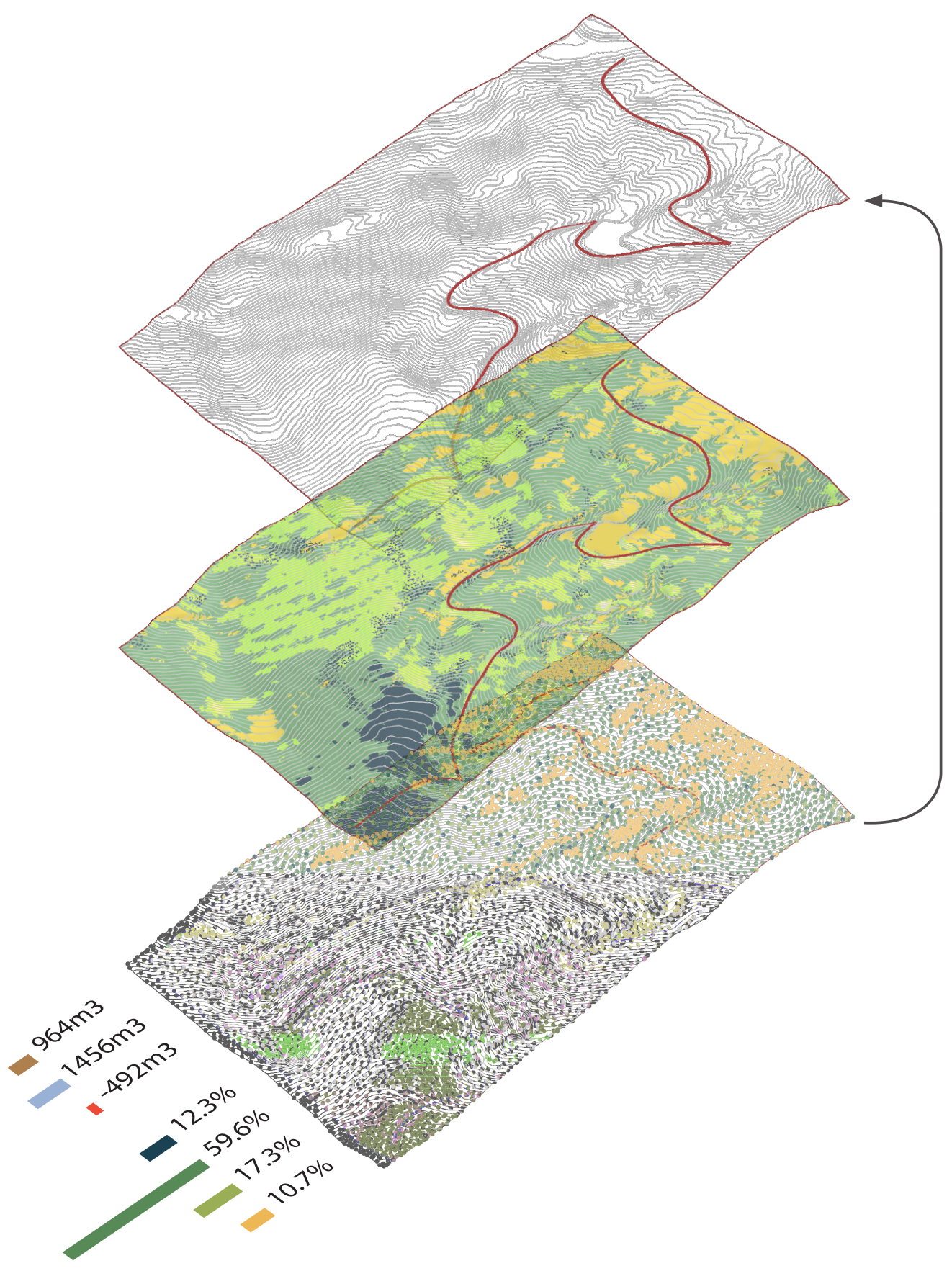

Figure.2.29. Path test, variation 3. 


\section{Study 2 - Conclusion}

Approaching the second study, there was a struggle to apply the concepts of the principal the parameter and the logic. Partly, the linear thinking that was so prevalent in Tory Street could not be applied so well in Moa point. The openness of the site forced an open mind, the scripted strategies became more compartmentalised and purposed opposed to branching logics enclosed by a two dimensional street plane.

The idea of toolmaking was key to this study. A variety of tools were created to achieve the common goal of pathway design in a coastal hill site. Domains separated the vegetation pattern on site, setting thresholds at points of difference. For instance, whether gorse was extending into the valley system, or if it was strictly on the ridgeline.

Through the tools created in Grasshopper, the entire workspace became. As it was decided early on that it was important to maintain a pull and push model, the tools created reflected that. Naturally they developed into tools of feedback all pertaining to slope. The path reflecting variations of difficulty and the terraforming reflected change to vegetation pattern. Working within these feedback loops the iteration process was guided through response to pulling or pushing of the path. While being a very informed conceptual process it does become very easy to focus on the data; the slope of the path or the effect on the vegetation and not the design of the path itself. This being the biggest critique that the study exposed.

Creatively however, this process does achieve a sensitive and attentive process of conceptual development. While still struggling to engage with the qualitative, the process instead thrives in the quantitative. This partly to the nature of computation. The augmented workspace allowed me to connect with condition of site much easier than I have experienced with plan or section, crediting the feedback loop model. Yet not taking the study to a resolved design state may have hindered a true critique of creative engagement.

To summarise the key points of this study.

- $\quad$ The mesh geometry is very versatile in its ability to represent topography. It can be used as a mediator to have other geometry projected onto it. The simulative model was possible through the dynamic relationship between the points and the mesh.

- Small and tactical tools with specific purpose were vital to engaging algorithmically in an open site. Scripting language must be known beforehand in order to think about how to solve the problem. 
- $\quad$ Personalised/augmented workspace allows the software to be customised to suit the needs of the site. Maintaining a pull and push modality encouraged a feedback model encouraging a sensitive and incremental flow of conceptual development.

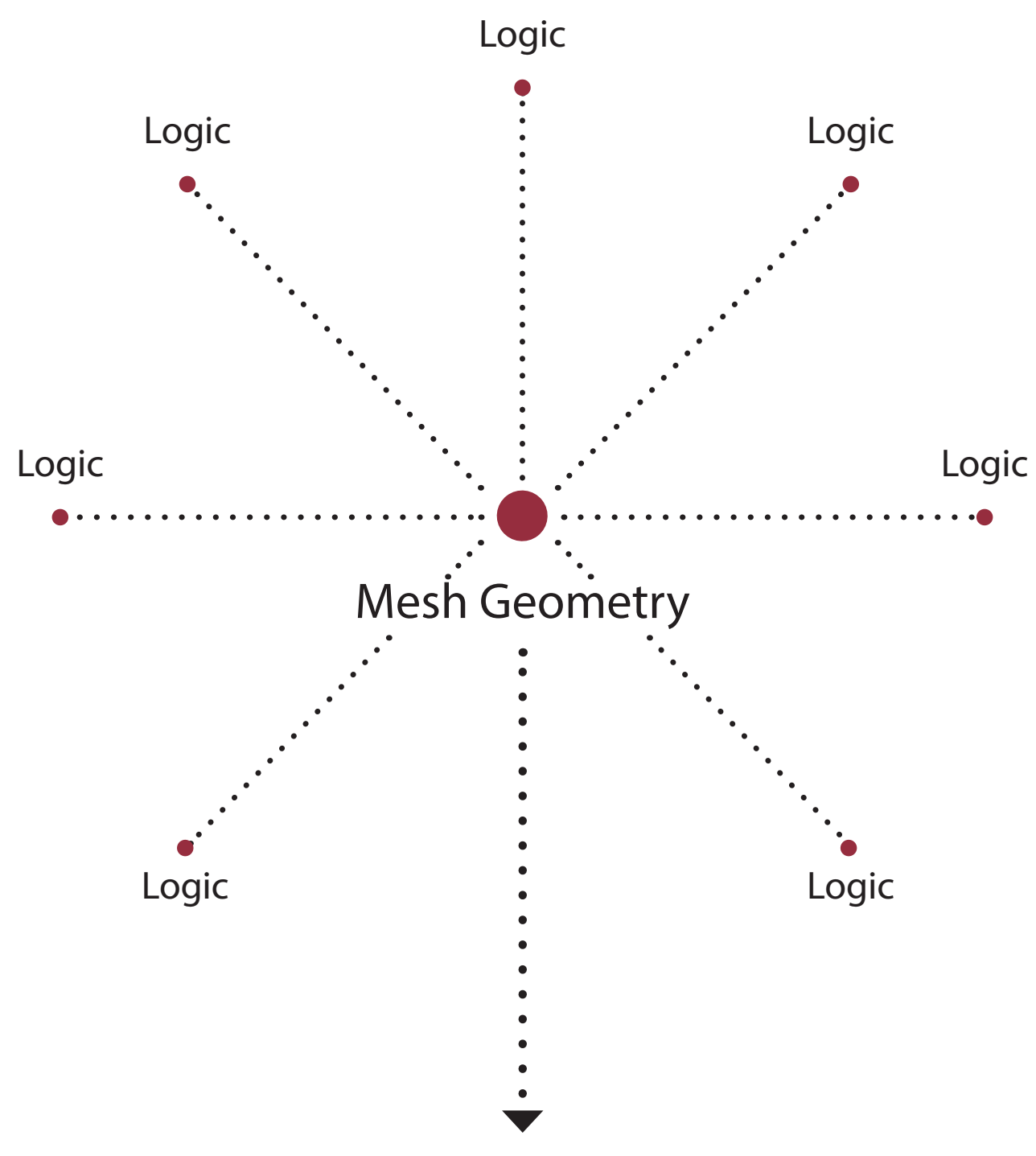

Feedback

Figure.2.30. Diagram of script structure. 


\section{Critical Reflection}

The two studies were purposely opposed in terms of condition and context. This in turn meant that parametric design strategy required different approaches to both sites. While the outcomes differed greatly, the difference in itself proved the flexibility of algorithmic thinking. In preparation for the third study the two prior were compared and evaluated in order to establish a path of action.

\section{Resistance}

The literature noted the ability to engage with parametric software as one of the primary arguements of the resistance to parametrics in landscape architecture. The fear mostly attributed to the creative engagement that is supposedly lost when using these tools. Baring in mind that these arguments tended to target CAD software, the notion yet naively sweeping parametrics as a whole.

While the first study aimed to establish basic principles for algorithmic thinking in a landscape architecture context, the level of detail in the design outcome was not high enough to make conclusive statements about the creative engagement of the tool. Although operating on sliders can be somewhat tedious and disengaging in itself, as sliders operate on a scale between a maximum and a minimum meaning multiple sliders are needed to shift an element in multiple directions. Being able to think in Grasshopper components and in parameters was not yet intuitive, although noting the existence of a hierarchy gave an initial glimpse into how parameter thinking might guide design thought in landscape architecture.

The second study explores a pull-push mode of engagement. This personalisation of Rhino through toolmaking, meant the tools could be tailored specifically to the needs of site. The flexibility of this is vital in landscape architecture as there are an overwhelming amount of site issues that we contend with. A landscape architect adapted to algorithmic thinking withholds a multi-tool vastly different to conventional toolsets. These two studies showcase two approaches to toolmaking, still, they only scratch the surface of what algorithmic toolmaking can produce.

\section{Paradigm}

The Principal, the parameter and the logic were three fundamental principles for guiding the development of the conceptual tool in the first study. The principal was naturally discovered and somewhat anticipated when firstly approaching the site as it defines a starting point for designing. In the initial exercise of going to site and perceiving it with Grasshopper in mind (as it was understood at the time), it was instinctual to look for limitations in regard to either moving an existing element or implementing new design elements. The building façades are obvious examples of limitations, restricting design action to the street realm. 
Logics were described to be actions, design moves, clusters of components that make a design action work. Logics essentially are the cogs that make the overall script function. A logic in the case of study two might refer to a whole tool such as the slope checker. In study 1 it was used to refer to a design action such as the region.

Parameter thinking invokes a search for the extents an element can move or exist within. Applying this to Tory Street, the chicane could exist anywhere between the existing kerbs, the regions could exist anywhere between the kerbs generated by the chicane and the building façades, the parking could exist along the kerb of the chicane yet not cross the footpath or intersect the region. Each parameter considering the parameters of the previous logic. Again in study Two parameter thinking was applied with the use of slope domains, by figuring out where planting species existed on site, their position, assuming unified site conditions (wind, soil type, sun exposure) could be replicated by isolating slope gradients on a mesh geometry. In both cases there is a search for extents.

\section{Tool}

In study one, the tool developed into a conceptual generator, that ultimately functioned as a dynamic diagram that re composed the three explored elements of the street, parking, program/function space and street side elements/furniture. The tool aimed to be supportive of the conceptual process in its efficiency to re-jumble the elements given to explore multiple potential compositions of the street.

While study one developed this single tool encompassing all design issues and design biases at once the second study developed multiple tools, smaller but more tactful. The compartmentalisation of these tools augmented the rhino model space into a process of pulling/pushing and feedback. This approach was much more engaging as opposed to shifting sliders in Grasshopper. There was much more attention paid to the landform, the real-time regrading of the contours and the potential effects on the vegetation; given by metric feedback. While study one felt removed from the reality of site, study two regained this with the concept of feedback loop operations, supporting Cantrell and Meikes ideas. (Cantrell \& Mekies, 2018) 


\section{Interface}

Representation was a struggle in both studies. Coded methodologies tend to be tedious following a ground up approach, meaning basic geometries must provide foundation for larger more complex scripts later on. As these scripts can often get quite large; nicely detailed elements will often be too data-heavy for most computers to handle, especially when built into a script intending to maintain a dynamic and flexible nature. In study 1 this was found when trying to develop accurate models for the street side elements, instead rectangles and poles were opted for as they were easier to generate. Mesh geometries inherently are often difficult to process, especially if they are highly detailed due to the significant number of faces that require re-computation at every change made to the mesh. Projecting thousands of points onto the mesh, as was done in study two, had to be considered carefully. A balancing act between accuracy of planting density and CPU power was played. For retaining dynamic capability of both models, a significant level of abstraction was required.

Although this abstraction is not inherently a bad thing. For the benefit of toolmaking in landscape architectural practice, it is likely better to be swift in creating these tools, to be tactful like the tools developed in the second study.

\section{Forward}

At this point in the research, the qualities of toolmaking seemed the most appropriate to the landscape profession. Algorithmic thinking enables a sequential and fractal thinking, begging the questions of cause and effect, and in return asking the designer if $\mathrm{x}=\mathrm{y}$ then what? Taking this thought process onto site we can begin to build site specific tools around this concept. Geometric relationship structuring is about this, asking this question repetitively until a tool is developed.

The third study will explore this concept further to develop on the following...

- Undertake a higher level of complexity in script building and toolmaking to better understand the limitations of Grasshopper/ algorithmic thinking in regards to landscape architecture.

- $\quad$ Better conclude the relationship between representation in its importance to an algorithmic toolset.

- $\quad$ Re-evaluate the conceptualisation of the principal, the logic and the parameter in regards to landscape architecture thinking. 


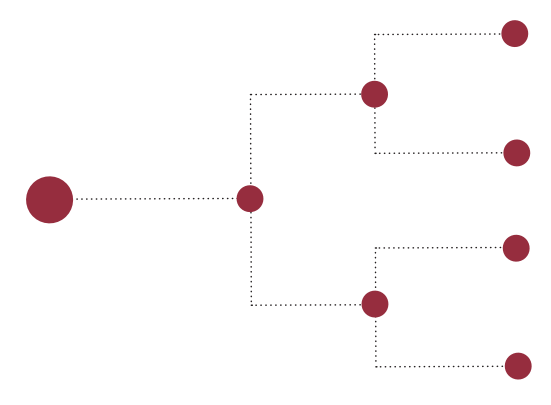

Figure.2.31. Study one script structure abstract.

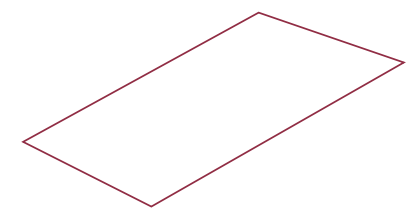

Figure.2.33. Study one street plane.

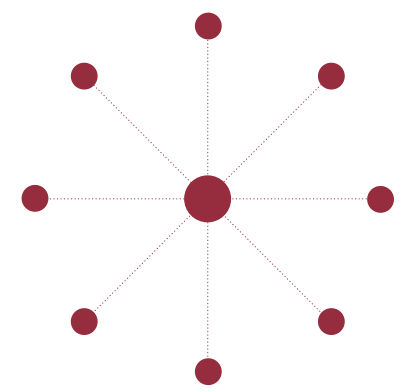

Figure.2.32. Study two script structure abstract.

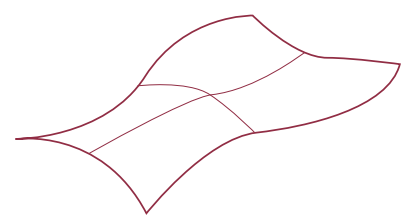

Figure.2.34. Study two mesh topography. 


\section{Study 3}

Porirua stream was chosen as the site of investigation due to a complex range of issues. Three were identified, each briefly understood from a parametric lens in sketch format, searching for methods in which toolmaking might be able to address or aid design within certain site specific issues. Site $\mathrm{C}$, concerning the connection of Cannons Creek and Porirua, and imposed on by transit infrastructure - was chosen as the final testing ground of the thesis to explore what has been learned from prior studies in a design scenario.

In response to site C's issues, the dynamic nature of Grasshopper was utilised to for relationships between designed elements and site elements in order to test spatial relationships and variation in a complex environment. A similar, but more evolved tactic to the first study.

The study is split into three of the four themes, paradigm, tool and interface. Paradigm addressing the thought procedures, alluding to the difficulties and strengths of parametric thinking. Addressing the complementation of sketching and re-conceptualising the meaning of the Principal.

Interface, addresses the engagement with the tool. Looking at the management of the script structure as well as the implementation of a separate sectioning tool that was incorporated to help perceive the design in a conventional way.

Lastly, tool addresses the use of the created script and how practically it suits the design brief of connecting the Porirua CBD to Cannons Creek through the reconfiguration of the interchange and railway.

\section{Study Three, Aims and Objectives}

- $\quad$ Reflect, revise and build upon prior conceived ideas of the tool, concerning the structure/hierarchy of components and algorithmic thinking.

- Utilise algorithmic toolmaking in response to a design problem in the context of Porirua.

- $\quad$ Reflect on hindrance and empowerment of algorithmic toolmaking in a design investigation. 


\section{Porirua Stream - Potential Issues to Explore}

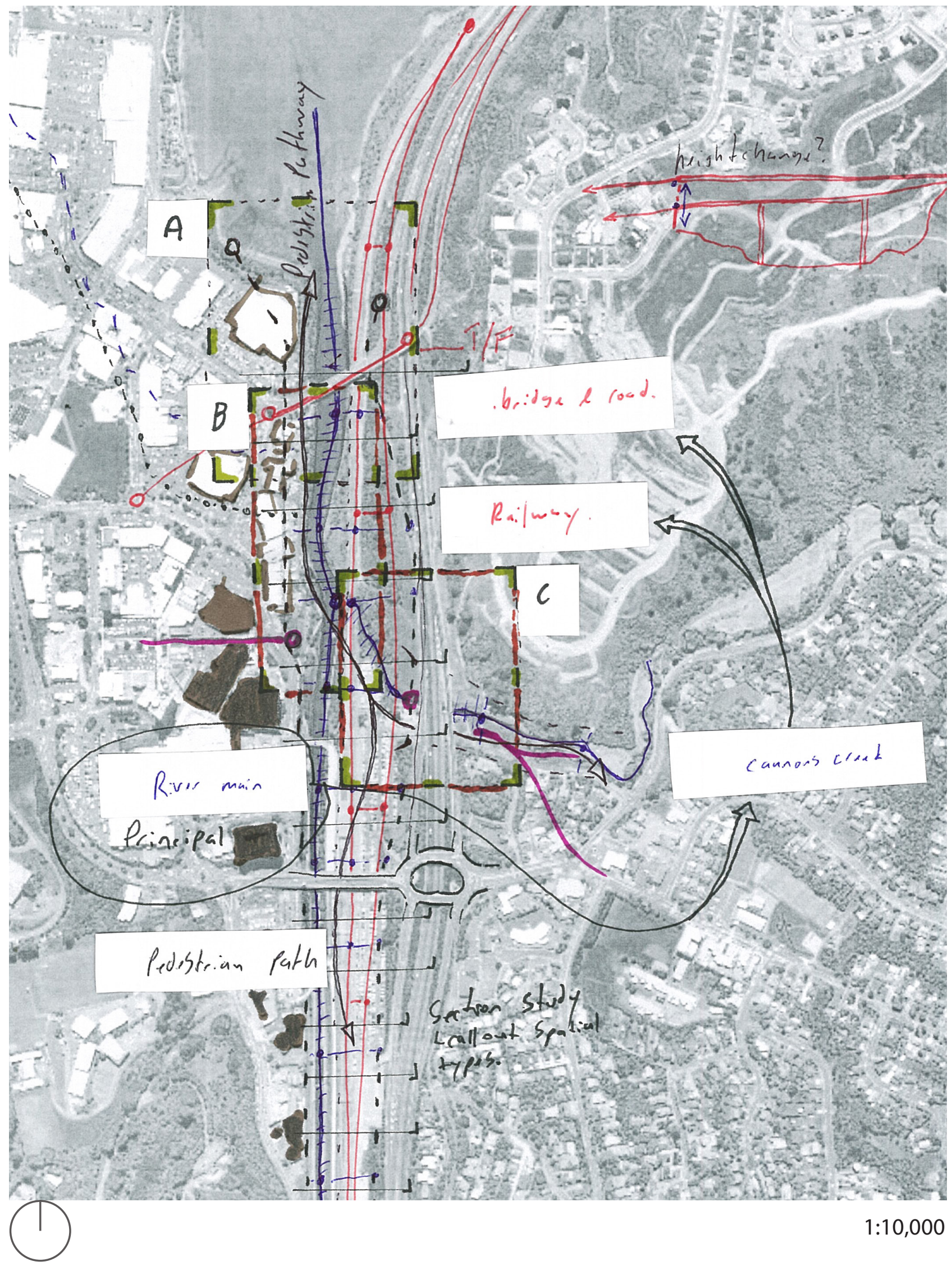

Figure.3.01. Porirua stream and potential sites investigation. 


\section{Potential Engagement A}

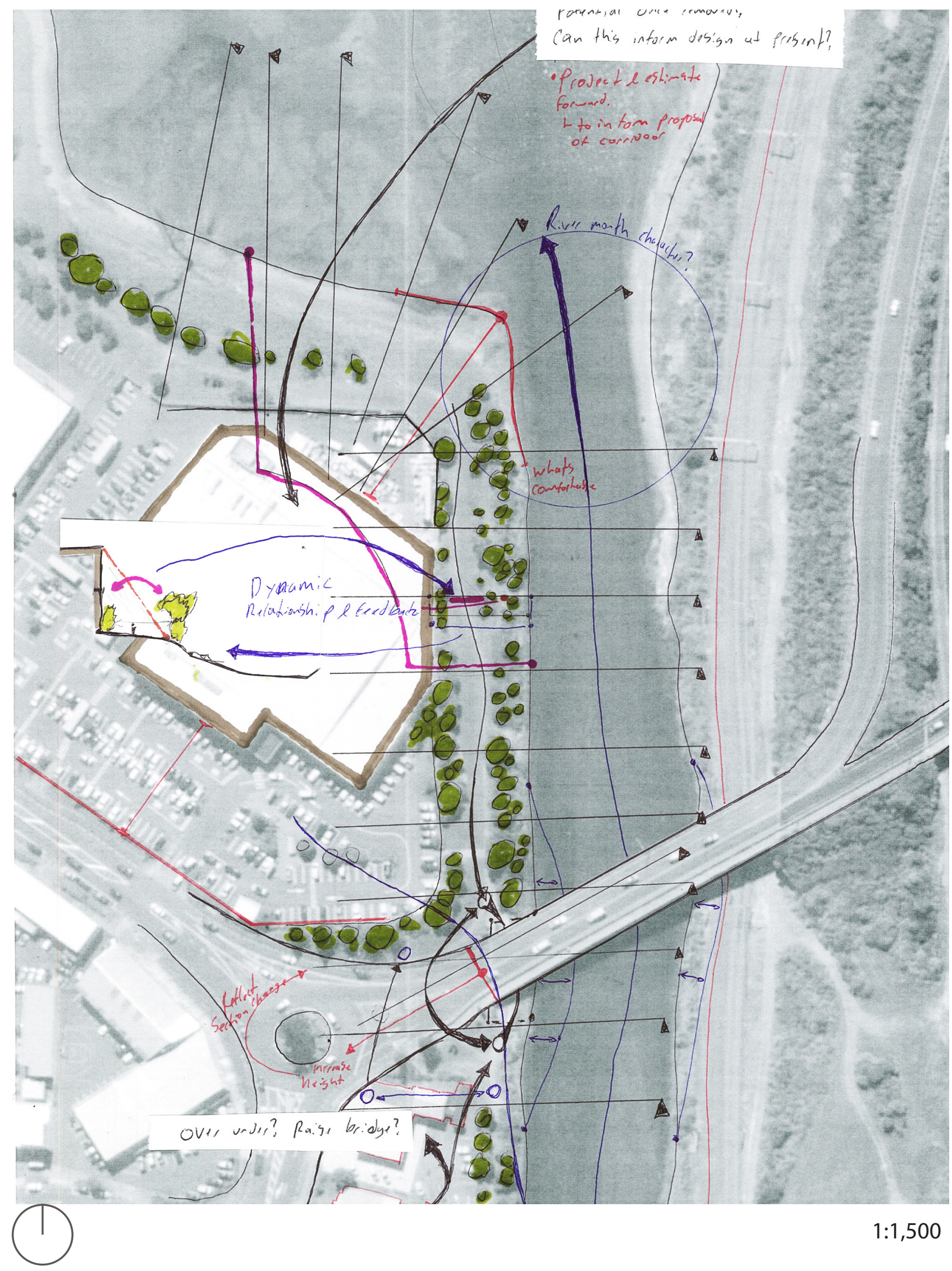

Figure.3.02. Porirua stream, site A.

Page 90 Study 3 
Site A considers the mouth of the struggle between mouth of the stream and the Pak' $n$ 'Save building. Because of this composition, walking the waters edge is a fairly uncomfortable experience in this area.

\section{Primary Issues}

- $\quad$ Pak'n'Save imposes the waters edge.

- Narrow claustrophobic path along stream.

- $\quad$ Limited space for design intervention.

Parametric Engagement

- $\quad$ Sectional analysis, the edge could be explored in an array of sections. Grasshopper Could enable a dynamic link between section, plan and $3 d$ to test for variations and aid in the composition. (This type of sectional study was adopted later in the study) 


\section{Potential Engagement B}

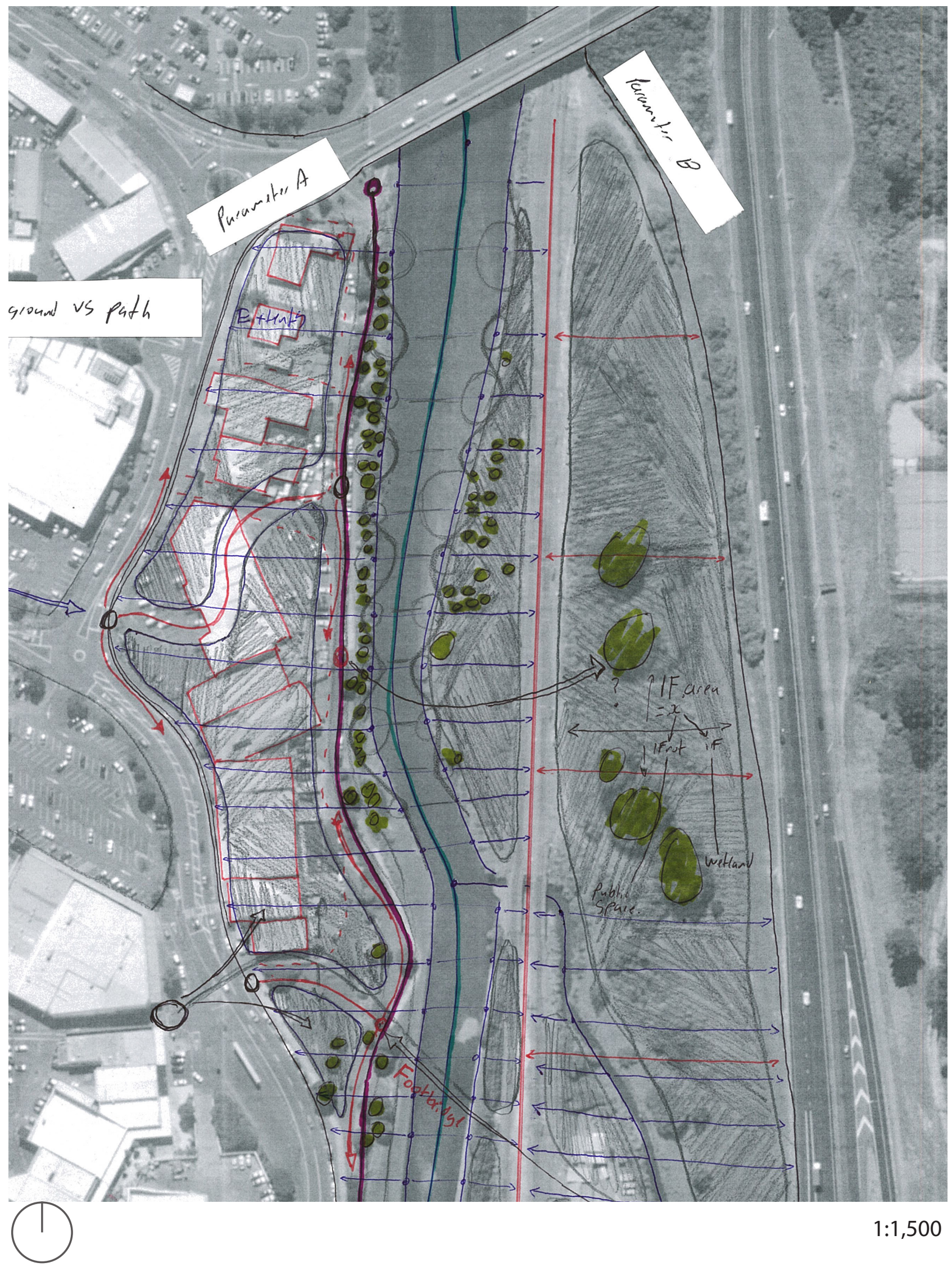

Figure.3.03. Porirua stream, site B. 
Site A concerns the middle of the stream and its relationship to the CBD. Currently the stream as a whole is poorly integrated however being situate between an active train station and a mall there is ample opportunity to improve connections and cross-flows of pedestrian life.

\section{Primary Issues}

- Disconnect from CBD to stream edge.

- $\quad$ Steep channel with little activity.

\section{Parametric Engagement}

- $\quad$ Pathway study, generating paths along and across the stream. The paths then might be used to divide the space into regions in which further logics could provide generative feedback, such as vegetation density and position or housing. 


\section{Potential Engagement C}

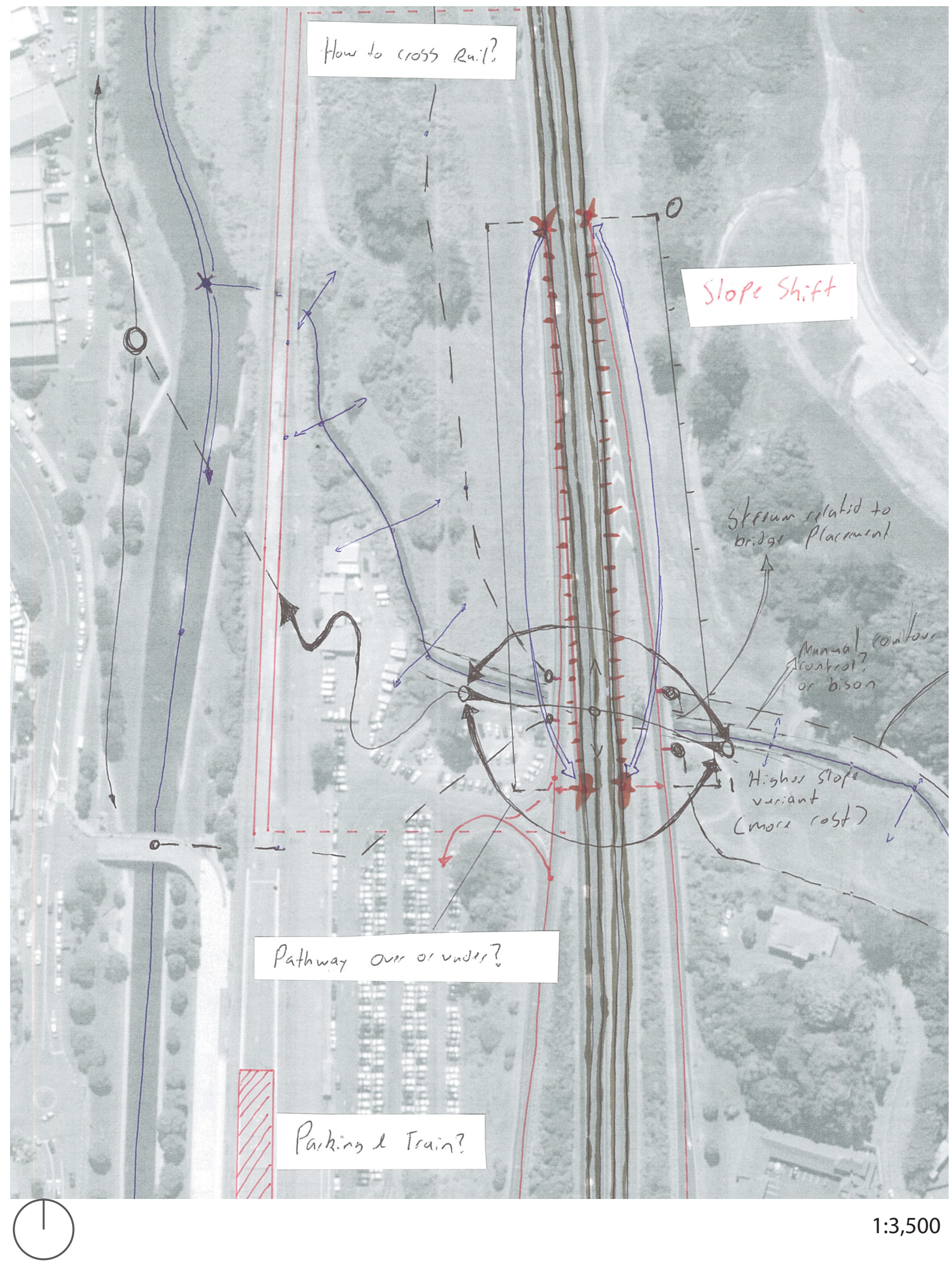

Figure.3.04. Porirua stream, site C.

\begin{tabular}{l|l} 
Page 94 & Study 3
\end{tabular} 
Site $C$ identifies the bridge and interchange that cross Cannons Creek. Cannons Creek as an area poorly integrates with Porirua, divided by heavy infrastructure. Connecting these two areas is vital for the growth and development of the Poriua region, especially Cannons Creek giving the prevalence of social housing.

\section{Primary Issues}

- Complex stream and infrastructure relationship. Difficult to change.

- Uncomfortable existing pathway from Cannons creek to CBD.

- $\quad$ Stream is neglected, not celebrated.

\section{Parametric Engagement}

- $\quad$ As the path from Cannons Creek to the CBD crosses the road, rail and river, it is a question of the horizontal versus the vertical. Parametrically the variables are clear and variations for potential paths could be explored deeply.

Under parametric paradigm the site questions the re-defining of vertical and horizontal vectors.

This study will explore the issues outlined in Site $C$ and pursue tactics for strengthening the connection of Cannons Creek and the Porirua CBD through the guise of algorithmic thinking.

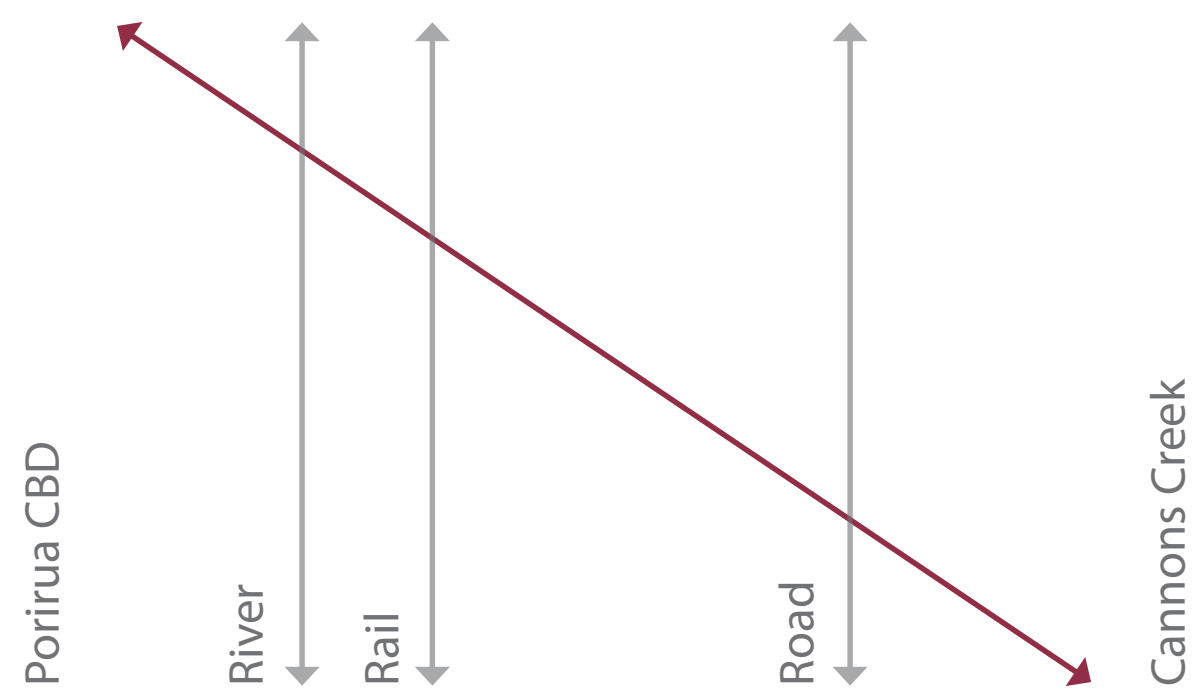

Figure.3.05. Site C, interpretation diagram. 


\section{Paradigm}

The definition of the principal shifted from its prior conception in the first study. As the study of Tory Street followed a linear process stemming from a single principal (the chicane), the term 'Principal' was used in the singular. This study however consisted of two principals, the interchange/ highway, and the railway line. These vertical vectors, by the definition of the principal, could not be affected by changes made to the stream or path but could, in turn, affect them.

Understanding this concept makes the practicality of the thinking clearer. Determining the principal gives structure to the interrogation of design which directly corresponds to the question which the designer is investigating. For instance, the question of this study is "How can we make a better connection between Cannons Creek and the Porirua CBD?" here It was previously stated that the problem is a contention of vertical and horizontal vectors. How might a path might cross these rigid pieces of infrastructure? This naturally led to seeking for flexibility and movement to rigid infrastructure, as to perceive new spatial potential and path amongst an existing complex composition.

Similarly the first study investigated the movement of the street, again the principal chicane street being a reflection of the question.

\section{Rigid Infrastructure}

The road infrastructure was firstly recreated in Rhino through a handful of points and curves to enable control over the flex and position of the interchanges. Also allowing for a switch between two lanes and four. Sketching was once again vital to the process and was continuously used to develop and workout logics throughout the process of this study. 
Two lower contours

$(A, B)$ determine the

parameter for the

stream to generate

its path, restricting

curvature and

variation/intensity.

\section{Contours flex}

based upon a point

adjustable along the

rail curve. (i)

The highway

functions currently

as fours lanes with an

interchange on either side.

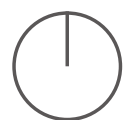

Rail

Road

Figure.3.06. Principal logics, Porirua. 
Figure.3.07. The positioning of geometries defines the form and movement potential of the infrastructure.
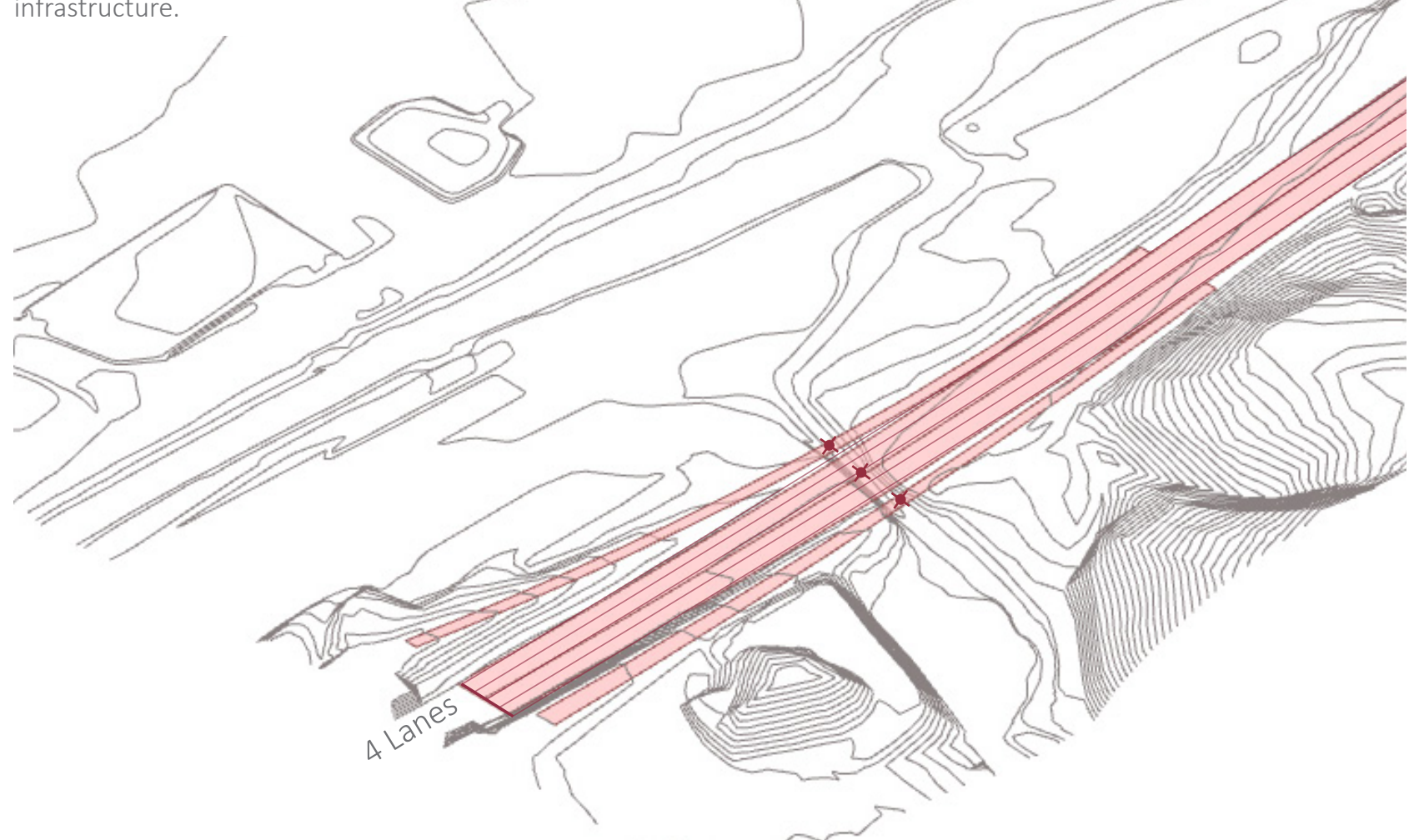

Figure.3.08. Raising the highway at the point of the underpass allows for the testing of vertical spatial qualities.

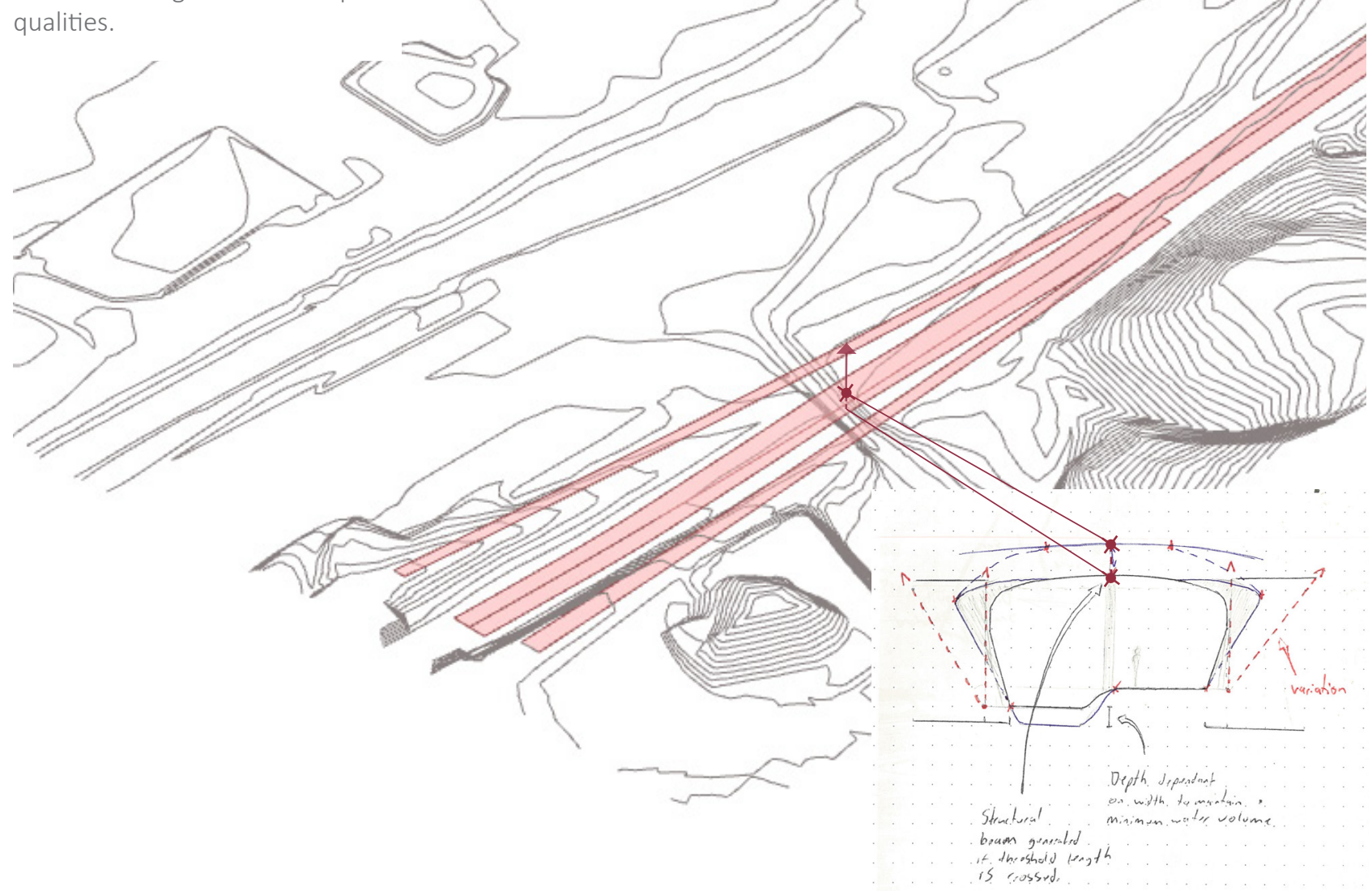


Figure.3.09. The position of the points define the bending moments of the curve, in the case of the interchange they were placed in relation to the underpass. This was so if they bent outwards, the underpass would benefit most from the exposure to sunlight.

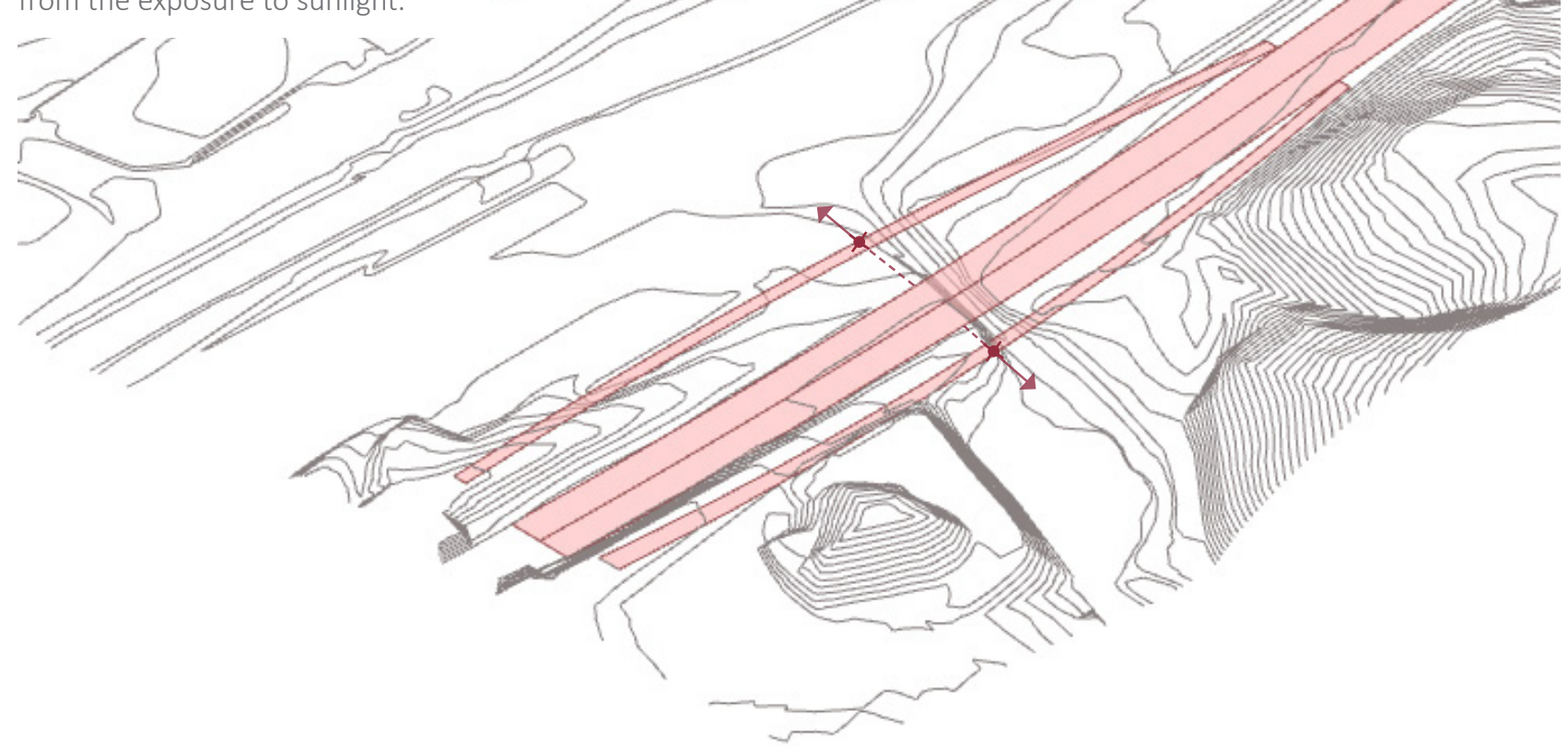

Figure.3.10. The road infrastructure might also be explored as a two lane road. Switching back from two lanes to four is available at anytime in the process thereafter.

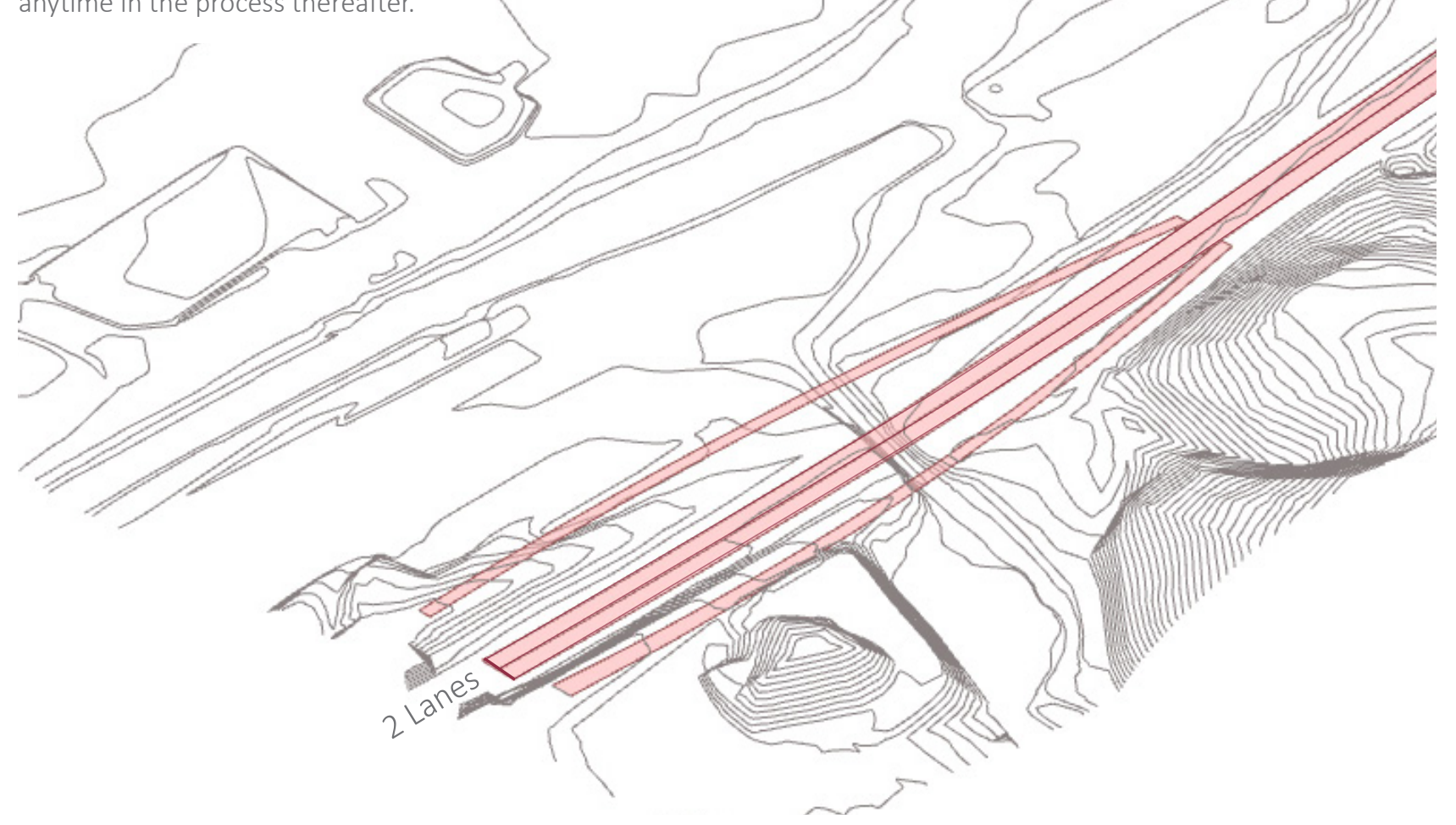




\section{Palette}

Every sketch was accompanied by coloured pens to prescribe method for translating sketch into points and curves. In the case of the underpass it was relatively simple to design as the edges were clearly defined by their strict relationship to the highway and interchange. From there, logics for the structural columns were programmed and then the detail and aesthetic was worked out by sketch. Vegetation points were generated within the openings between the interchanges and the highway.

Working through sketch to understand the relationship between the underpass and the highway and interchanges.
The underpass must address the horizontal vectors of the pedestrian paths and the stream.
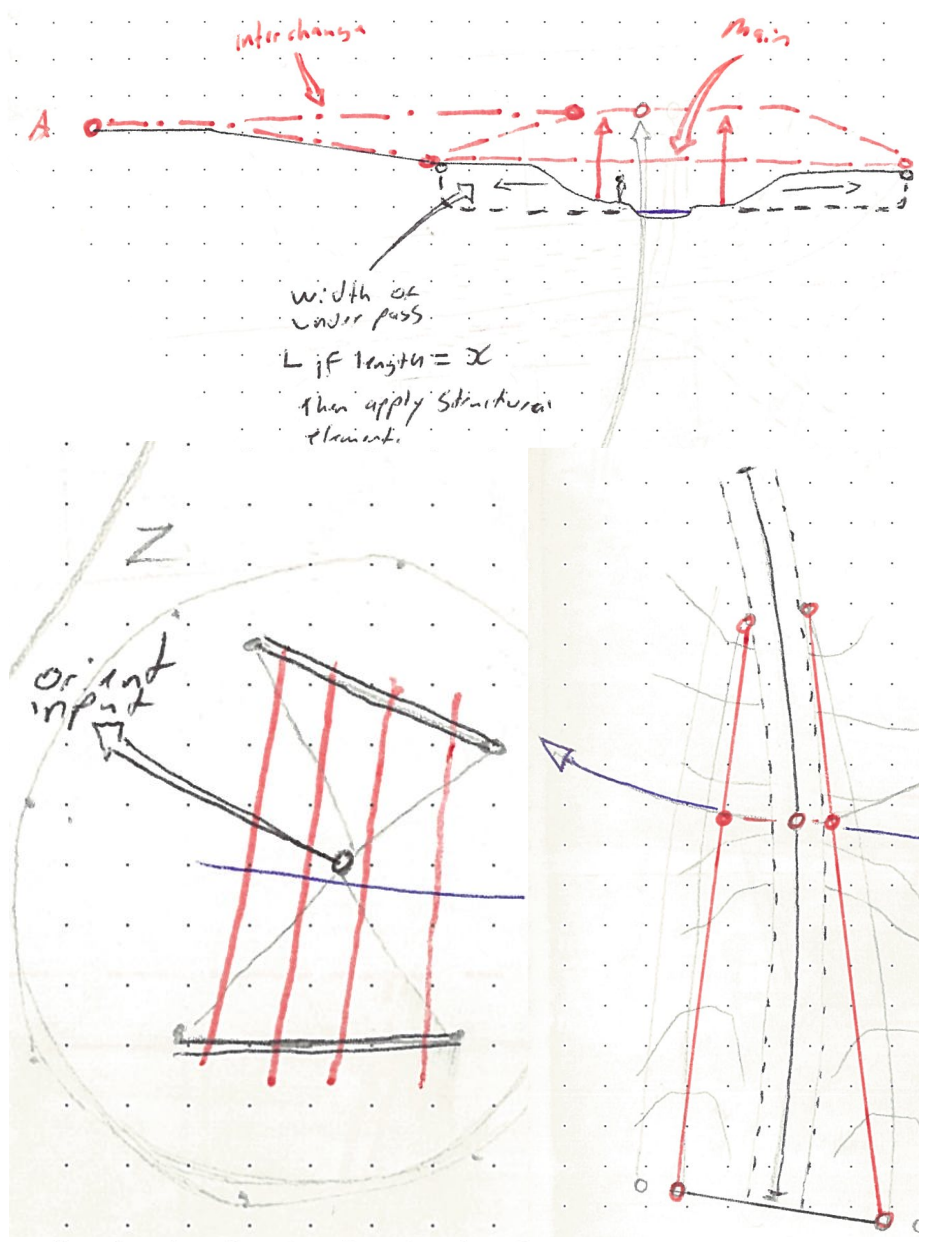

$$
150-240 m .(200 \text { Suggoster) }
$$

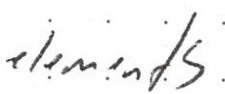

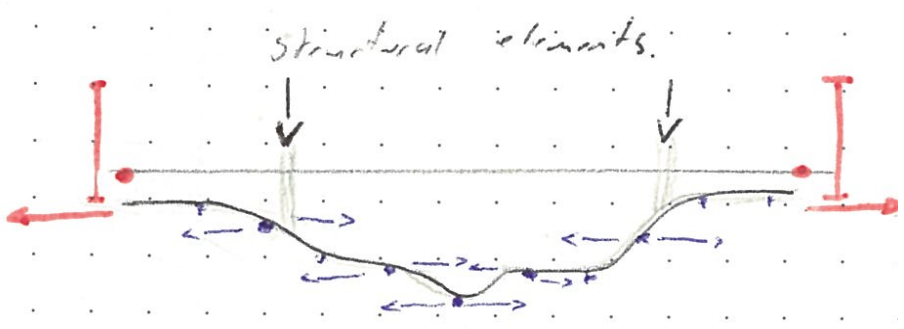

Figure.3.11. Sketch notes of underpass and road logics. 


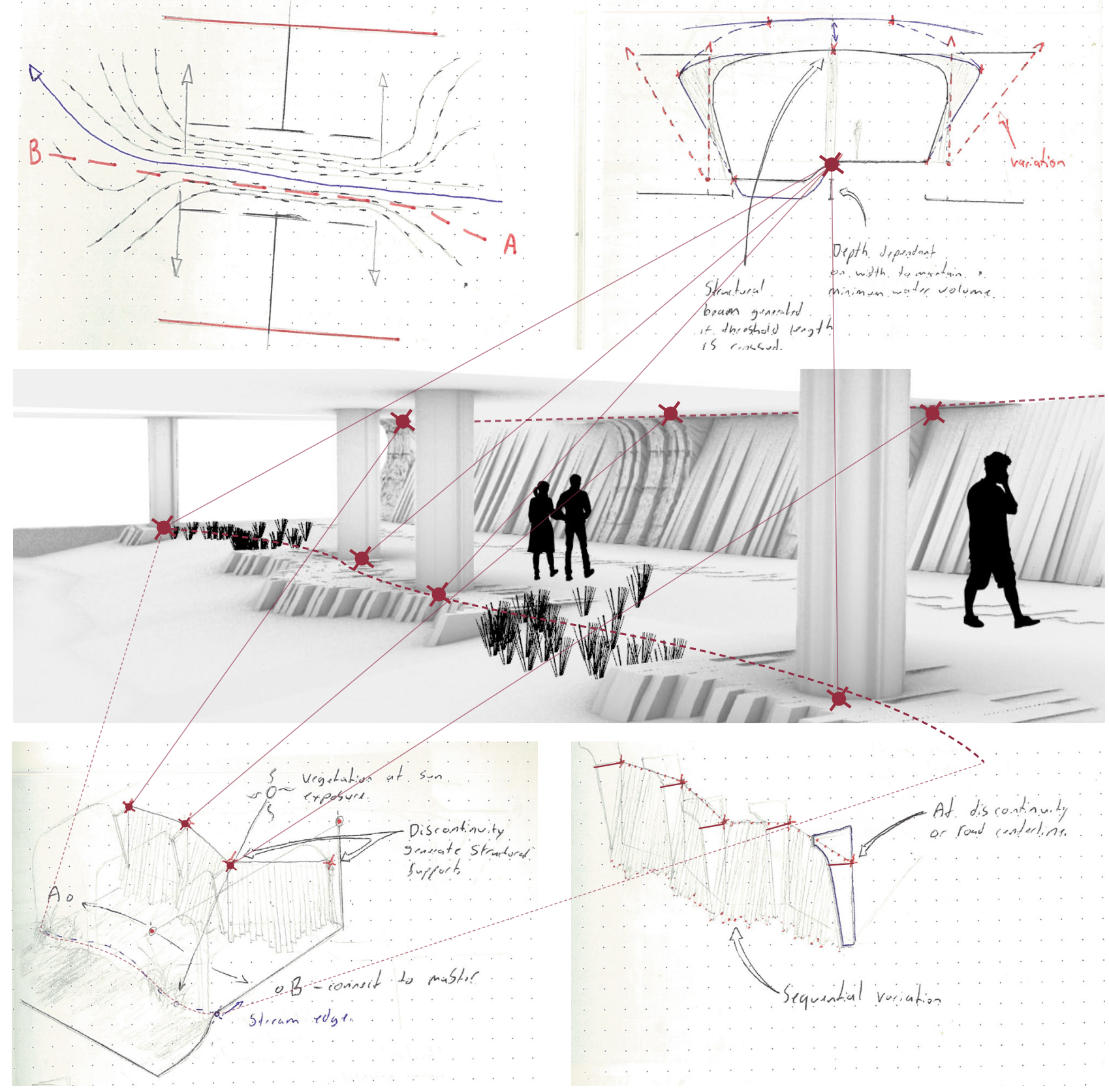




\section{Underpass Exploded}

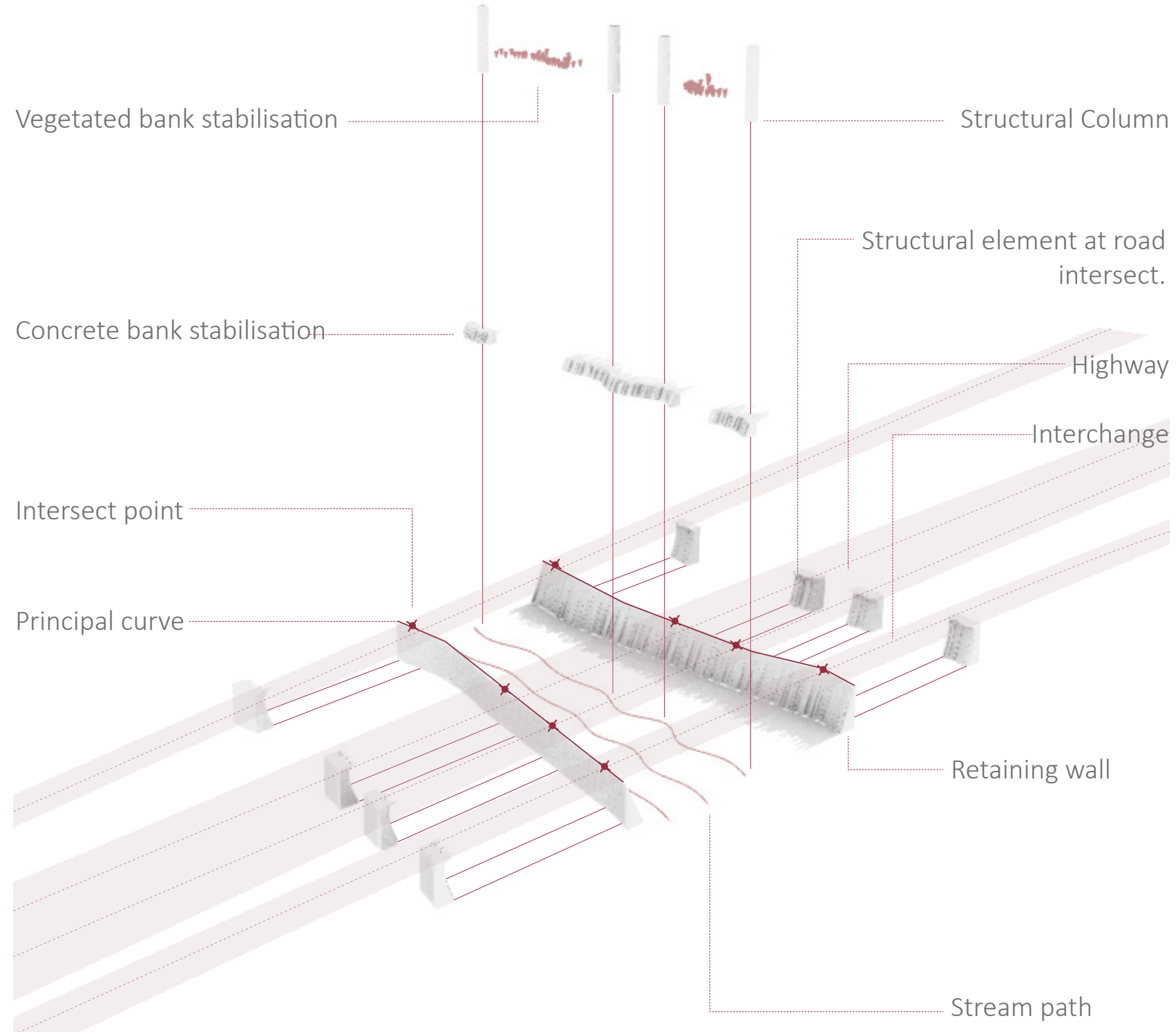

Figure.3.12. Exploded diagram of underpass.

Page 102|Study 3 


\section{Underpass Variations}

As the design embedded itself into a set of structural curves, alterations were quickly achievable by changing a few input values. Patterns in the retaining wall and paving could be intensified or homogenised, the underpass could fan at either end or be a long narrow thoroughfare. Structural columns generating at points exceeding a specified distance from side to side. Concrete bank stabilisation would occur underneath the road and vegetation would occur in the openings, the edge of the stream could also be altered in levels of intensity.
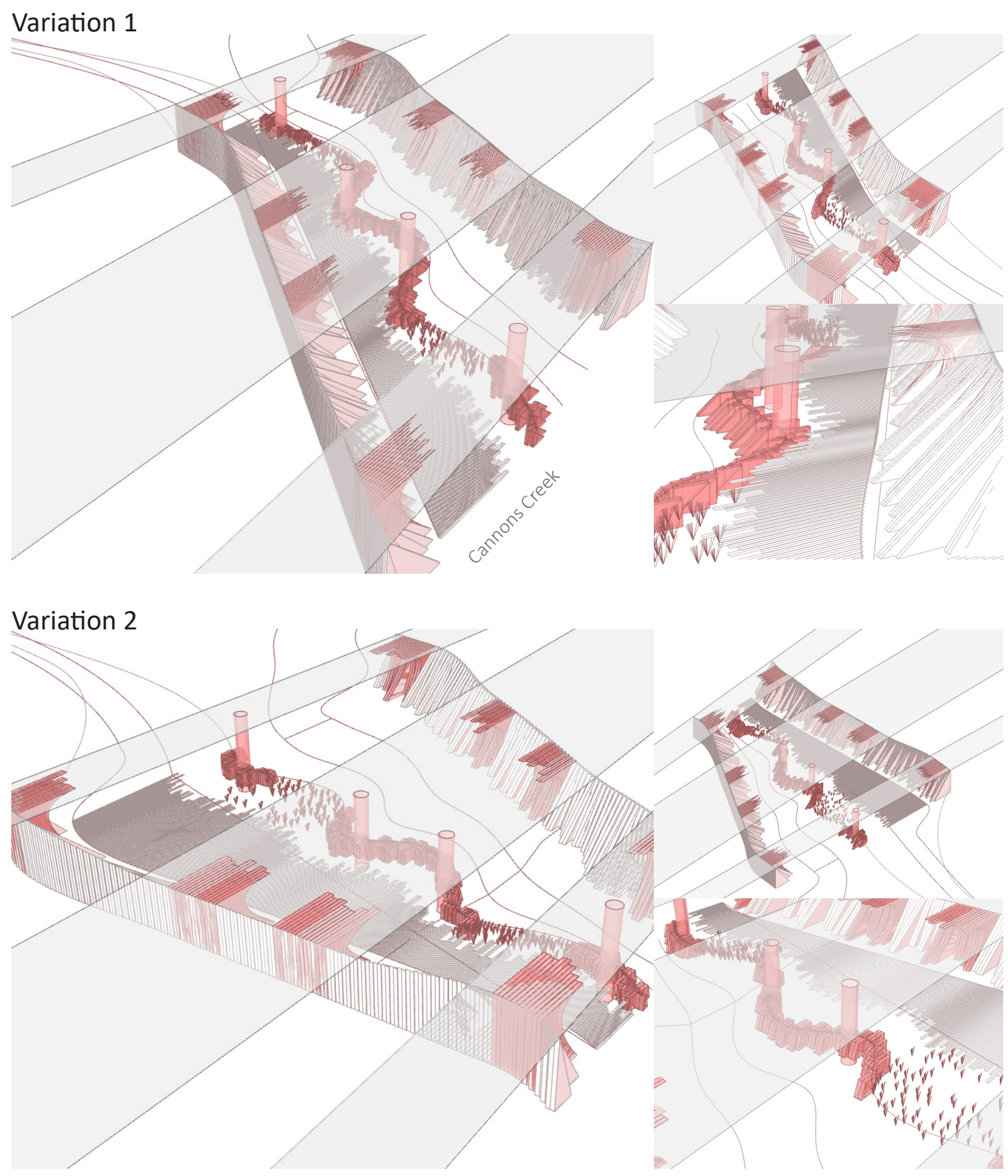


\section{Further Variations}

Variation 3, takes on a wide and linear form. Variation 4 widens the interchange from the highway, bottlenecking at one end. Variation 5 reintroduces 4 lanes to the highway, thus widening the underpass. Variation 6 takes a narrow form, removing the need for structural columns.
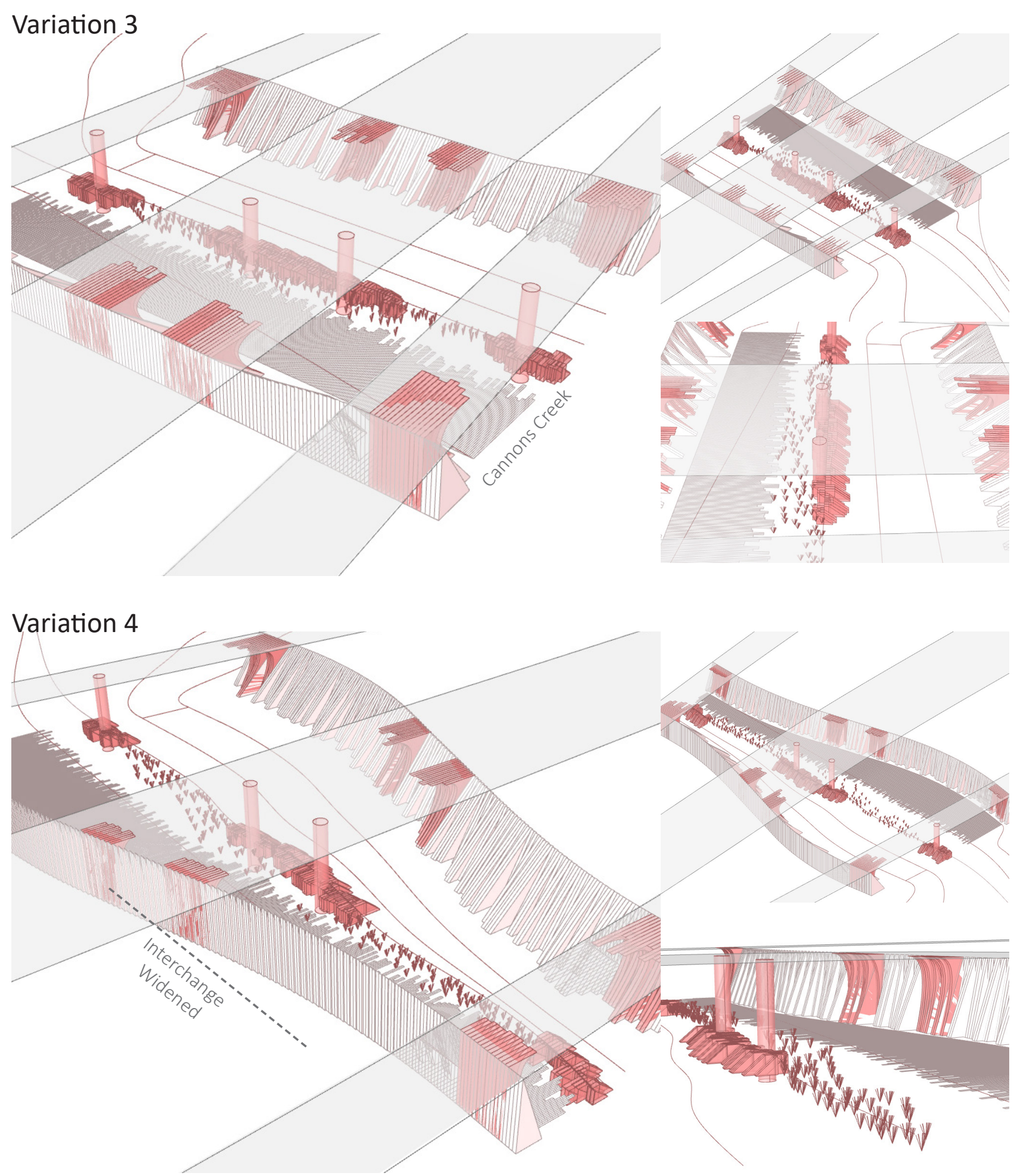

Figure.3.15. Variations of underpass.

Page 104|Study 3 

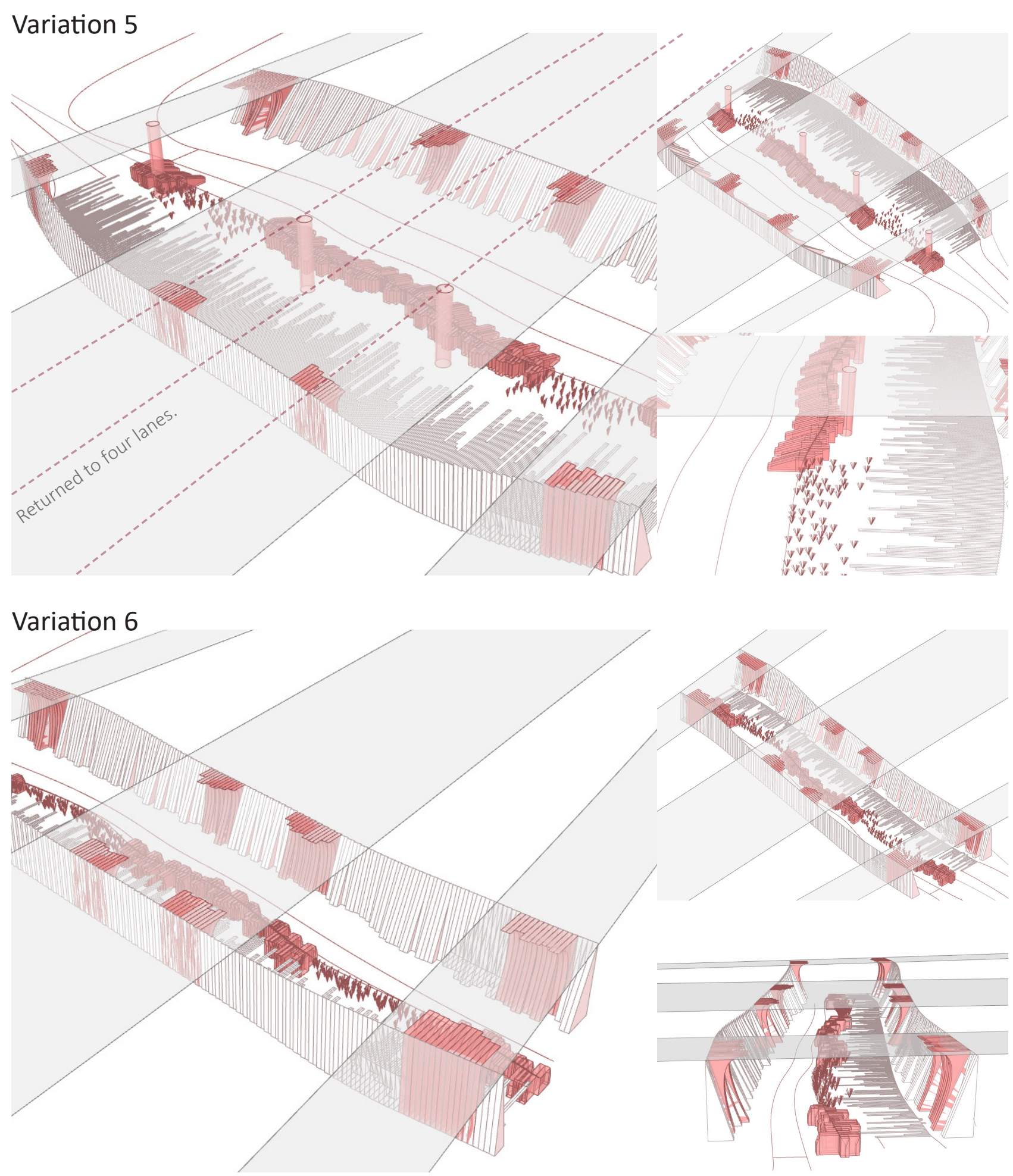

Figure.3.16. Variation 3 of underpass. 
1:100 Underpass Section

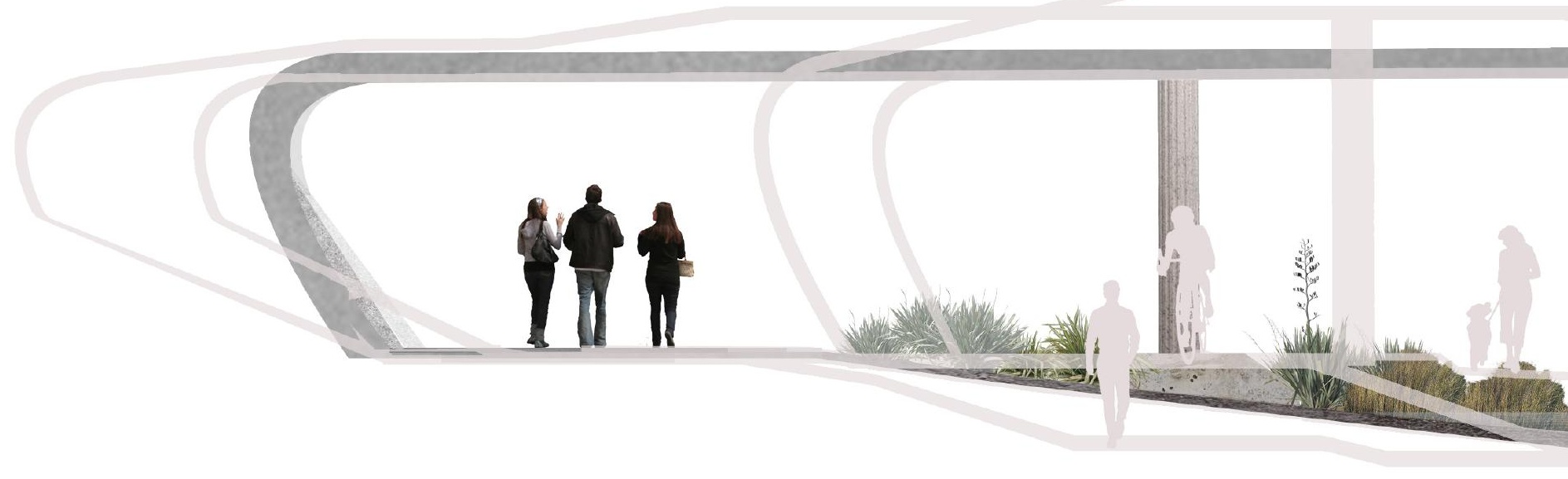

Figure.3.17. Section of underpass, showing alternative variation potential behind.

Page 106|Study 3 


\section{Generative Logics}

Generation in the sense of this tool is explored as two concepts, a randomised or patterned generation of form but also as a programmed design response to the exceeding of a threshold or condition of a given geometry. As variations are to be teased and manipulated, some responses need to be programmed with detail, such as the overpass responding to the proximity of the rail line. While a random generation allows for unexpected outcomes.

\section{Programmed Generation}

In two cases design response were programmed to the exceeding of certain parameters. In the case of the underpass, if the width exceeded 20 meters, structural columns were required. These columns would generated at the intersection of the road infrastructure and the stream edge. A bottleneck underpass form would only require structural columns at one end of the structure as can be seen in variant 4 of the underpass variations. (pg 100, figure 3.15)

The second case arose from the challenge of crossing the railway line. There were two foreseeable ways of crossing the rail, under or over. Rather than sticking to one trajectory, both options were explored with the use of programmed generation. If the rail was to be moved close to the underpass, it was opted that going under would be more suited as the rail is more intertwined with the infrastructural composition of the highway and interchange. If the threshold crossed the specified distance, the rail would raise 2.5 meters and the point geometry guiding the path would generate under. If the rail exceeded the distance, an overpass logic would generate, and the path would recognise the new reference points.

\section{Threshold Not Crossed Threshold Crossed}

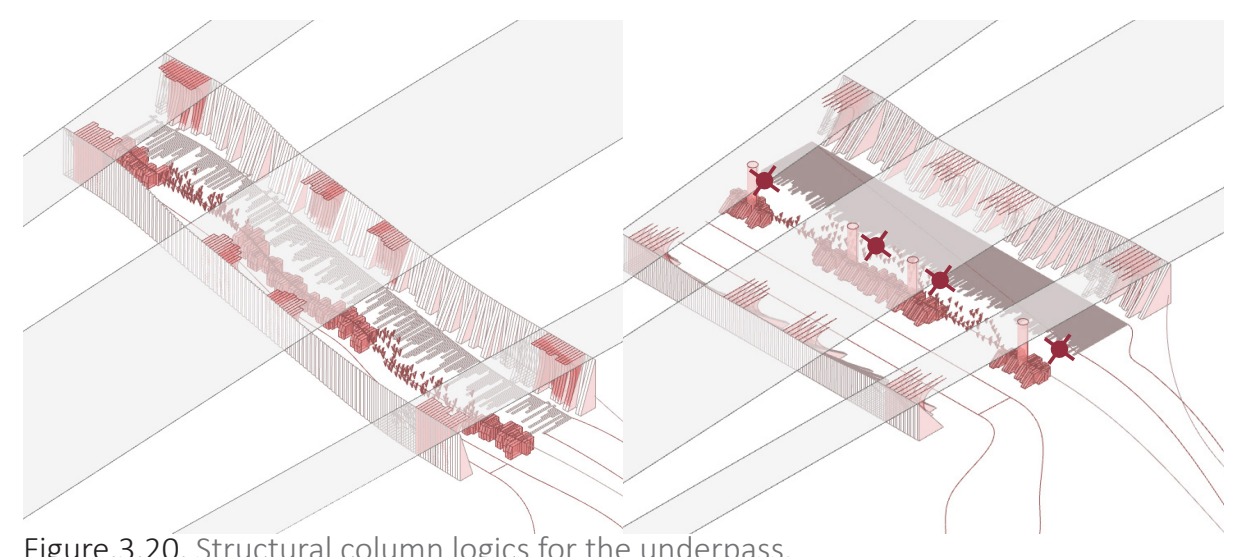

Figure.3.20. Structural column logics for the underpass.

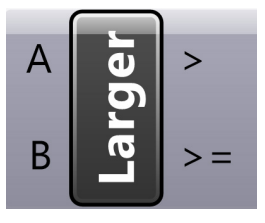

Figure.3.18. Larger than component, used to determine if a value is larger than another, used to figure out distance thresholds. 
Figure.19. Stream gate component, controls the flow of data through gates allowing for the programming of multiple design scenarios different if conditions. The true/false output of the 'larger than' component might plug into (G) true $=1$ false $=0$

Threshold Crossed
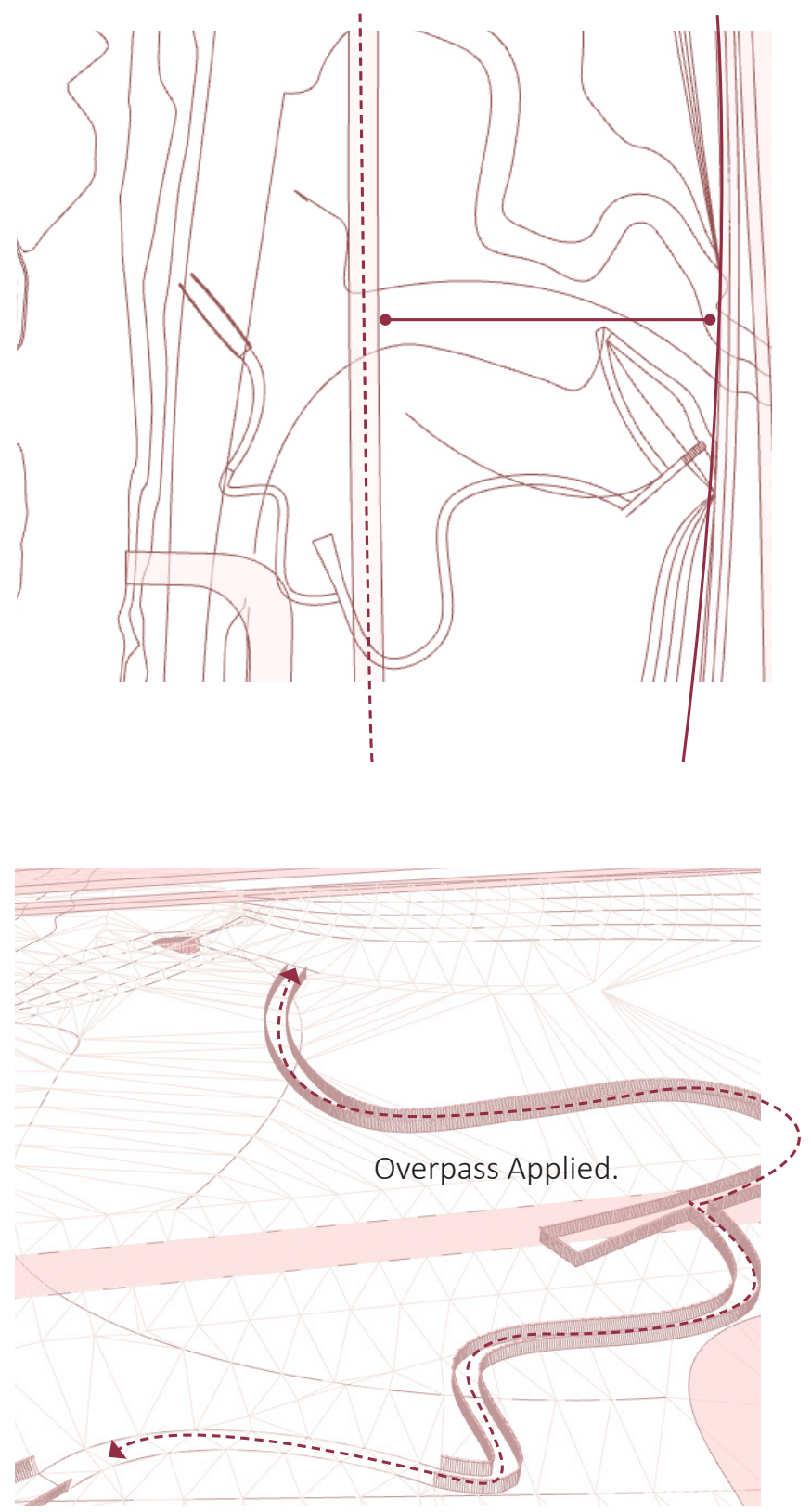

\section{Threshold Not Crossed}
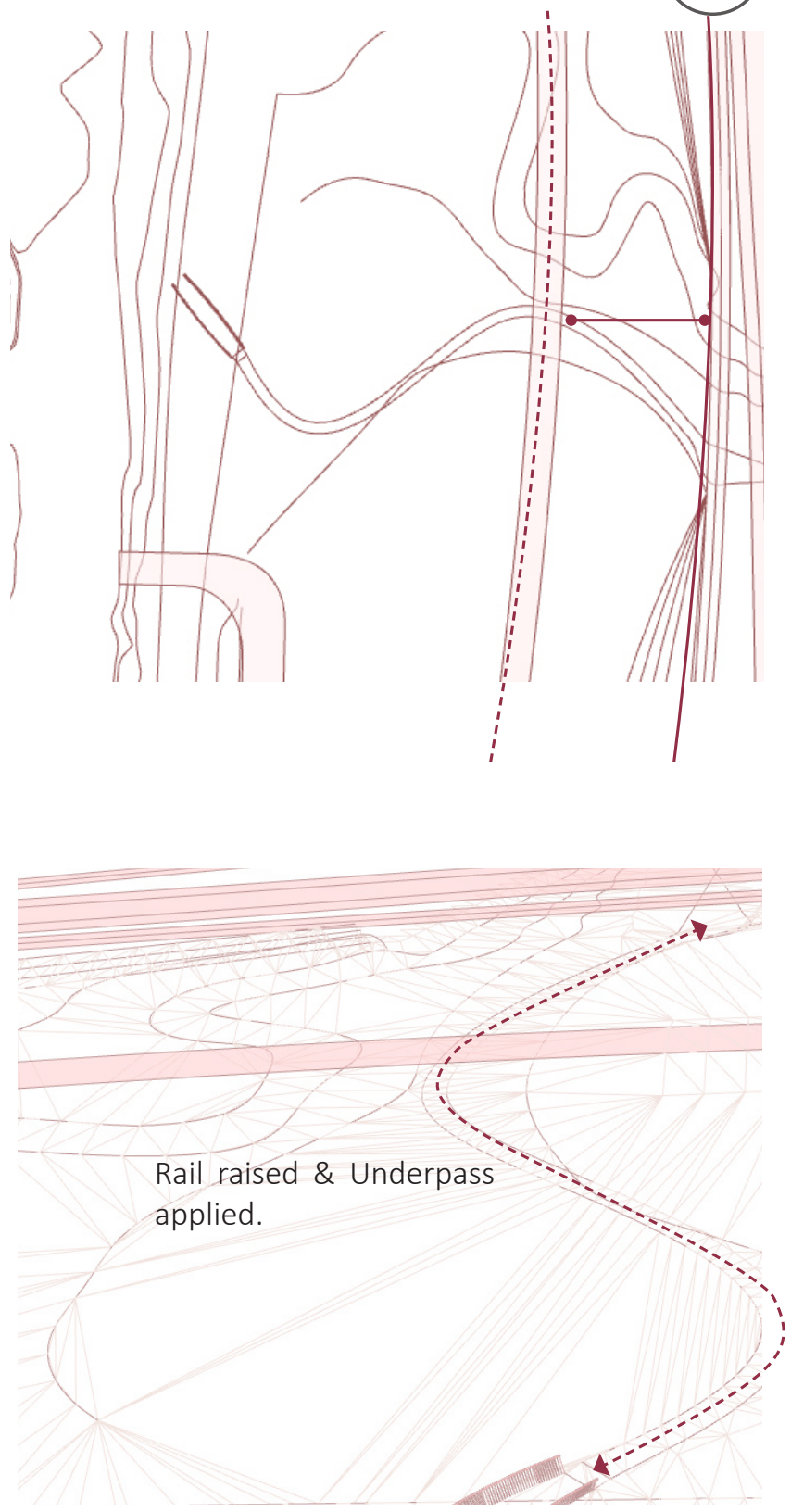

Figure.3.21. Overpass program logic. 


\section{Randomised Generation}

The generation of the stream was defined by two parameter curves (A \& B). The curves were divided into an equal amount of points on either side and the lines were then strung between opposing points. Points were then generated at random intervals along the lines, the intensity and variation of the stream could be controlled by allowing the points to generate along the full length of each curve. Alternatively, the stream could be straightened by restricting the length along the lines that the points could appear at. By stringing these points together, the path of the stream could be created. With the seed input of the jitter component (Fig.3.22)- generations of the stream can be explored quickly. This type of generation is much more randomised than the overpass and produced by adjusting parameters to compute a random stream pattern.

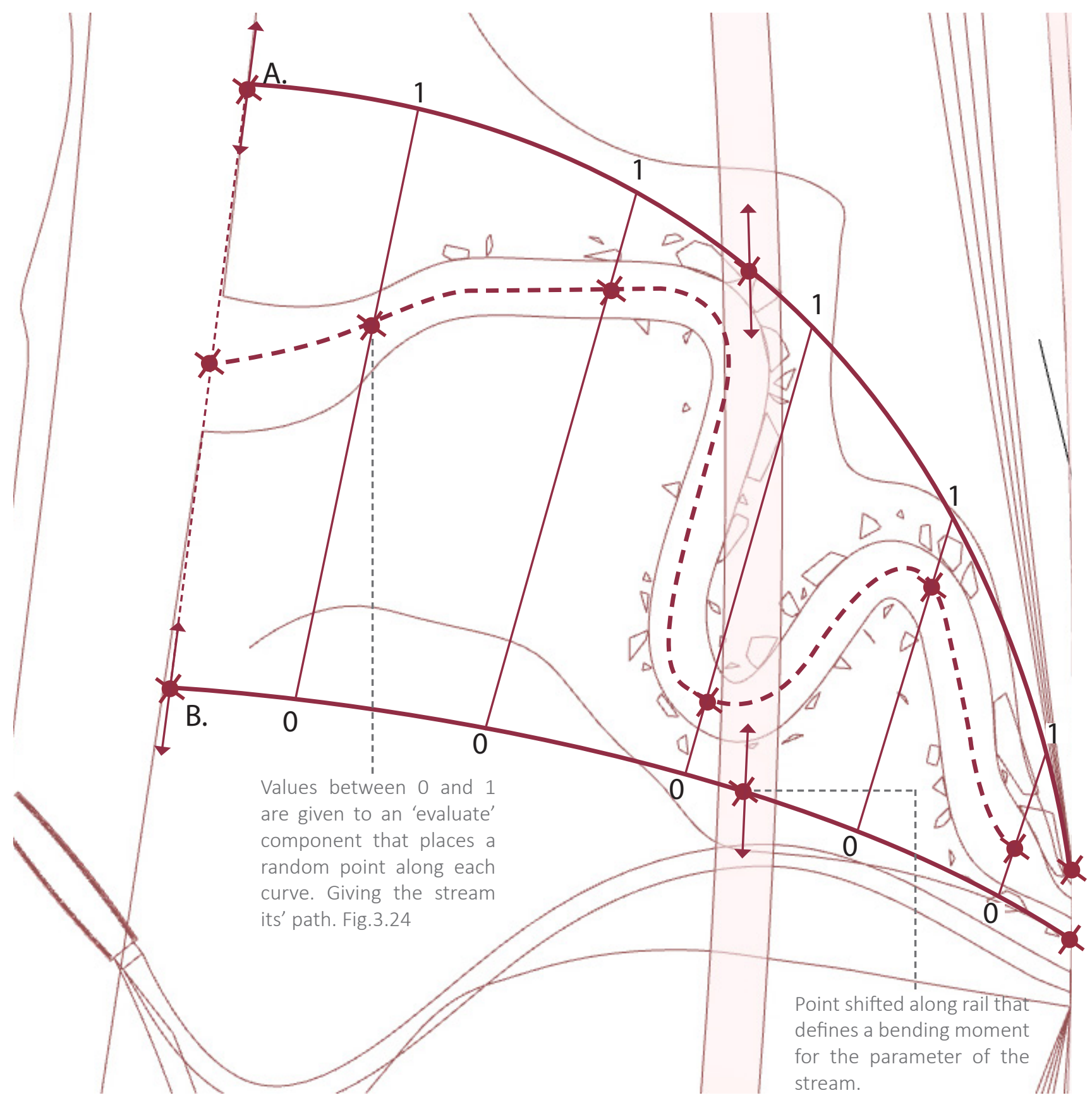

Figure.3.23. Stream generation, parameter diagram. 

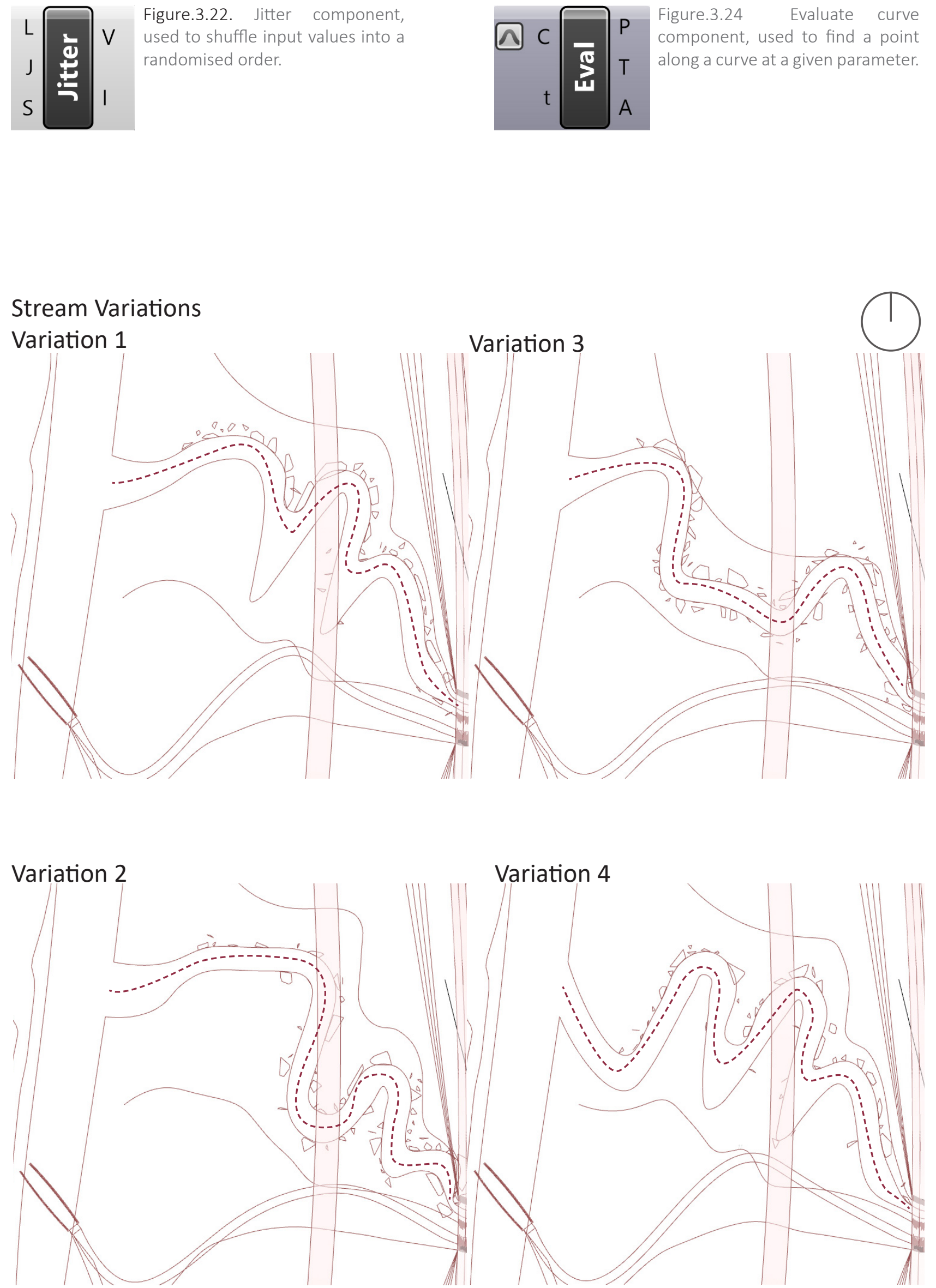

Figure.3.24. Variations of stream path generation. 
Variation 5

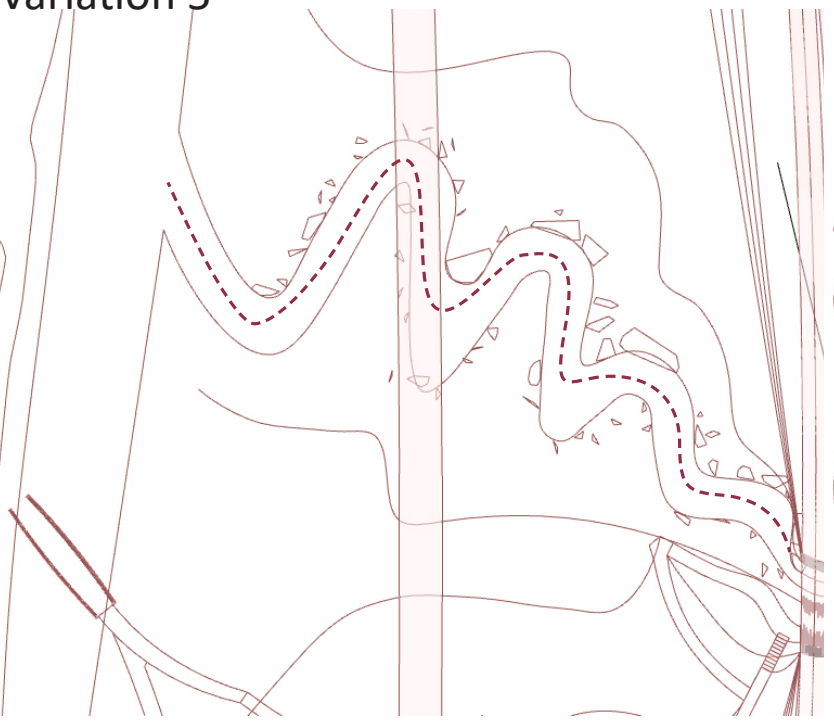

Variation 6

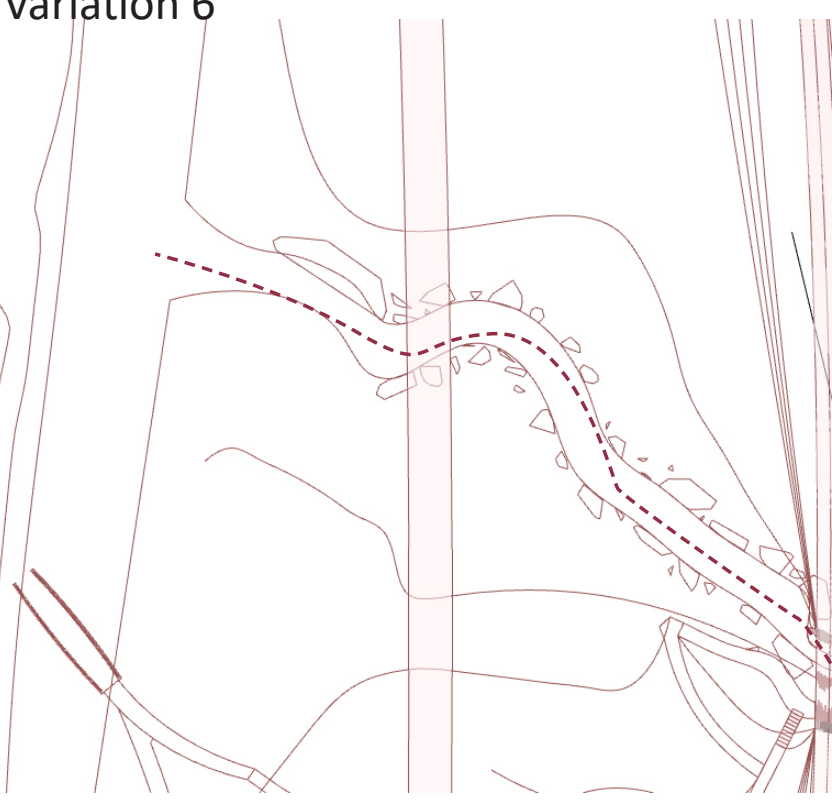

Variation 9

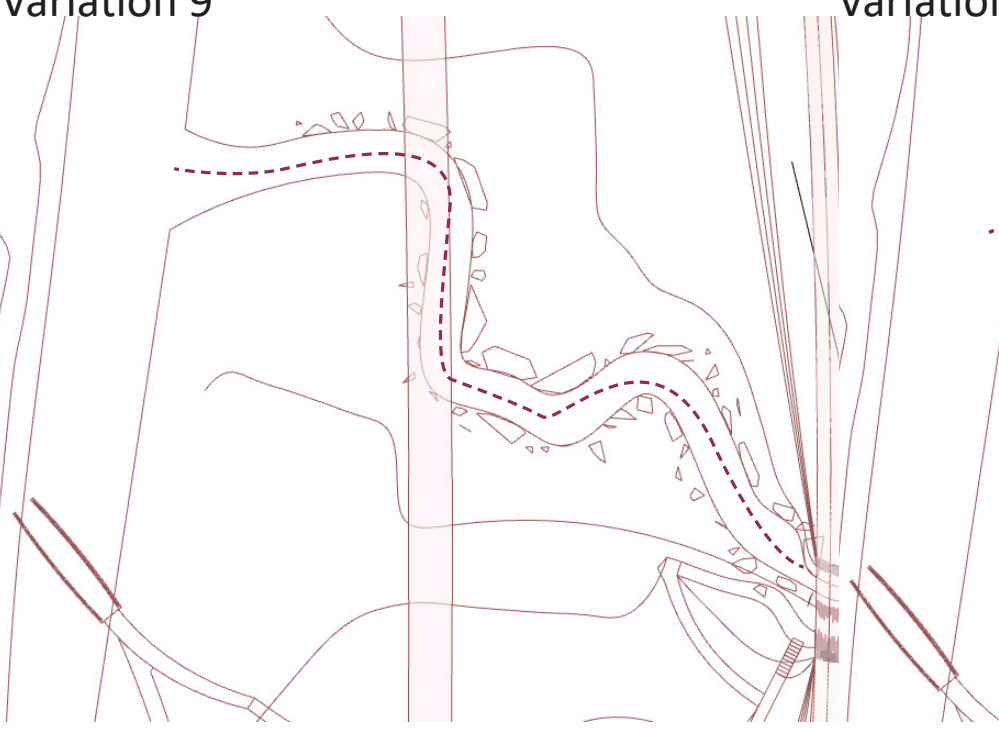

Variation 10

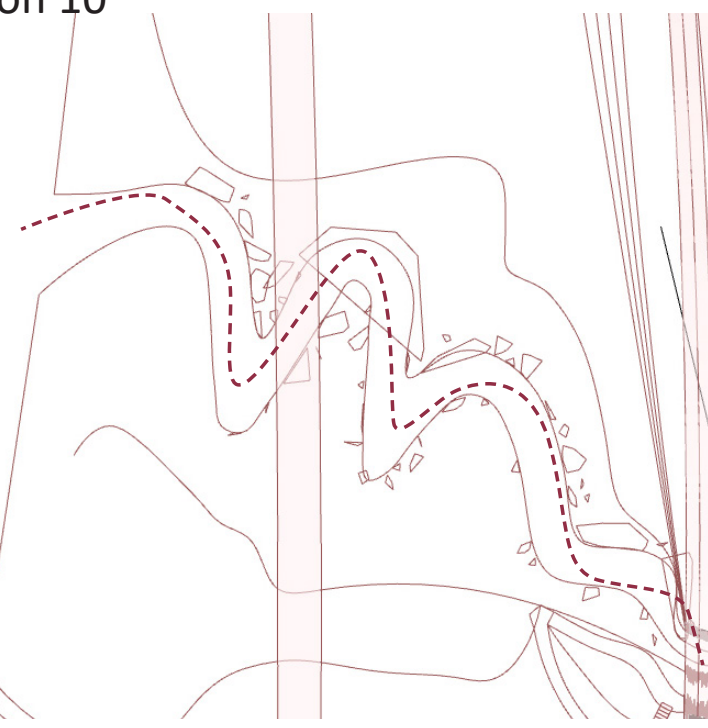

Variation 8
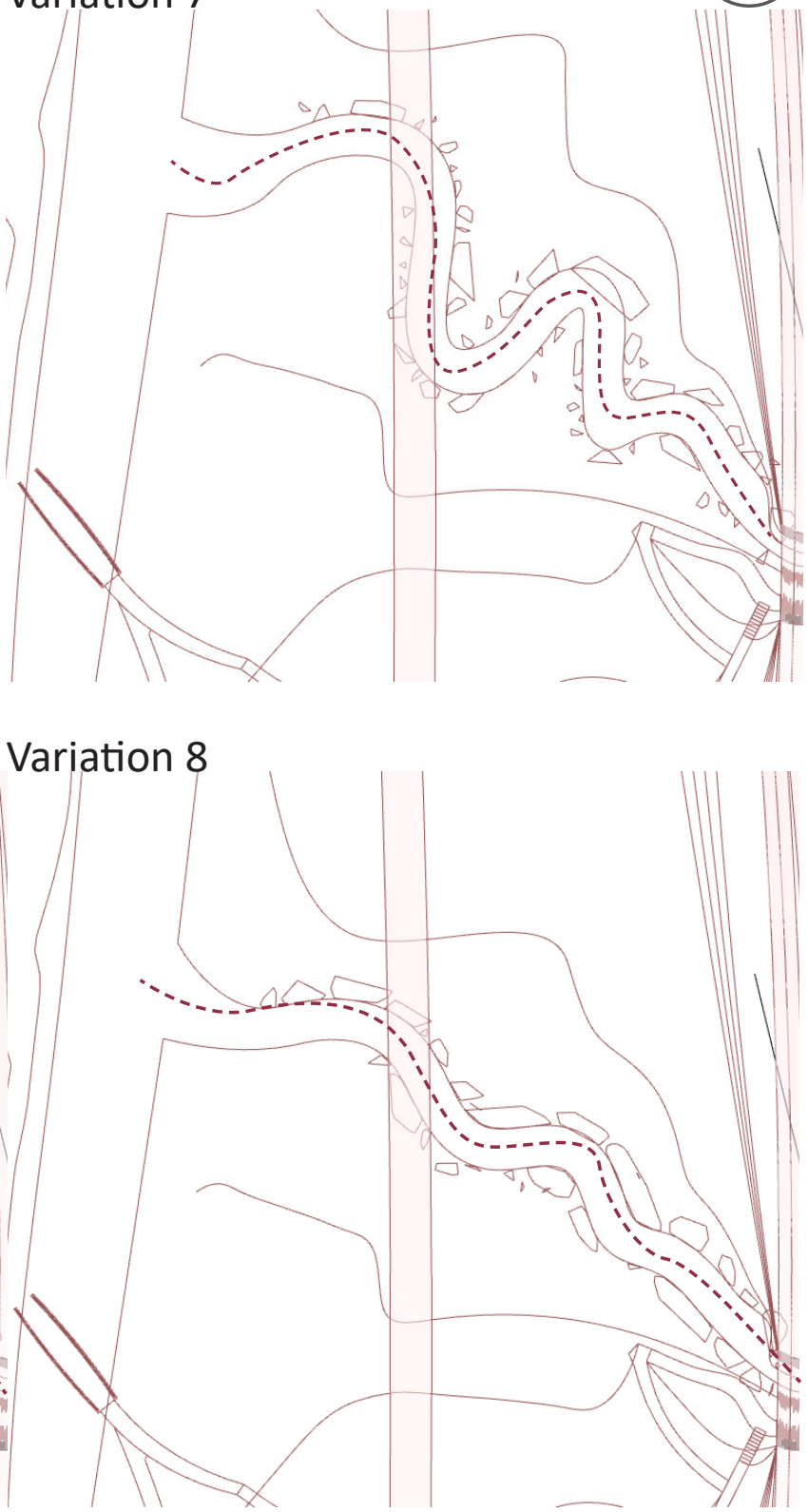

Variation 7

Figure.3.25. Further variations of stream path generation.

Page 112|Study 3 


\section{Bank Vegetation}

Randomised generation was used also to generate vegetation along the stream edge. Represented as either points or polygons, the shapes could be altered with the graph component shown in (Fig.3.27). With this generational tool, the vegetation could be scattered or clustered into large clumps, or a combination of both. In combination with different stream configurations, the vegetation can quickly be re-interpreted under these variables.

Vegetation clusters generate to form polygons.

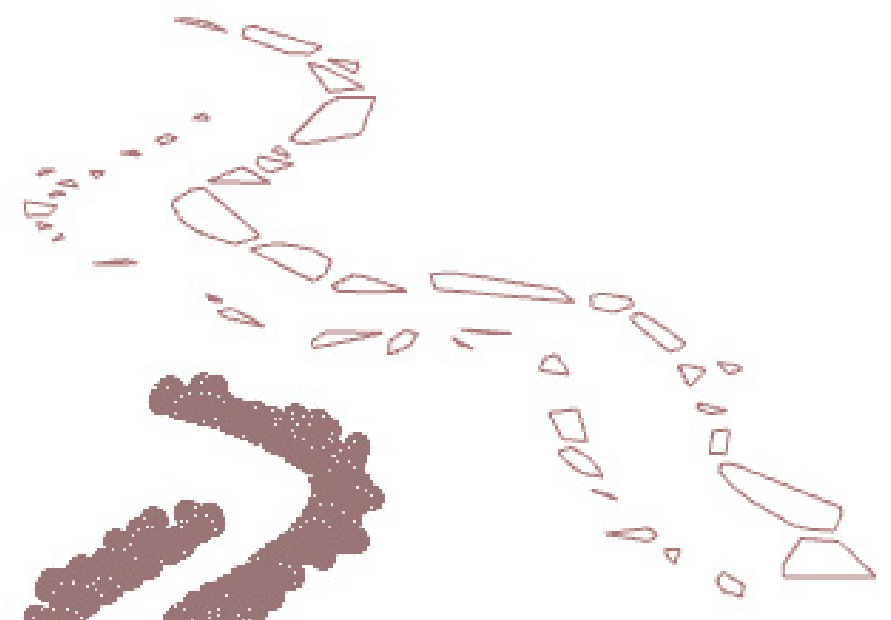

Points generated, 1 per m2.

Region determined by offsetting the stream edges by an adjustable distance.

Less/smaller Scatter
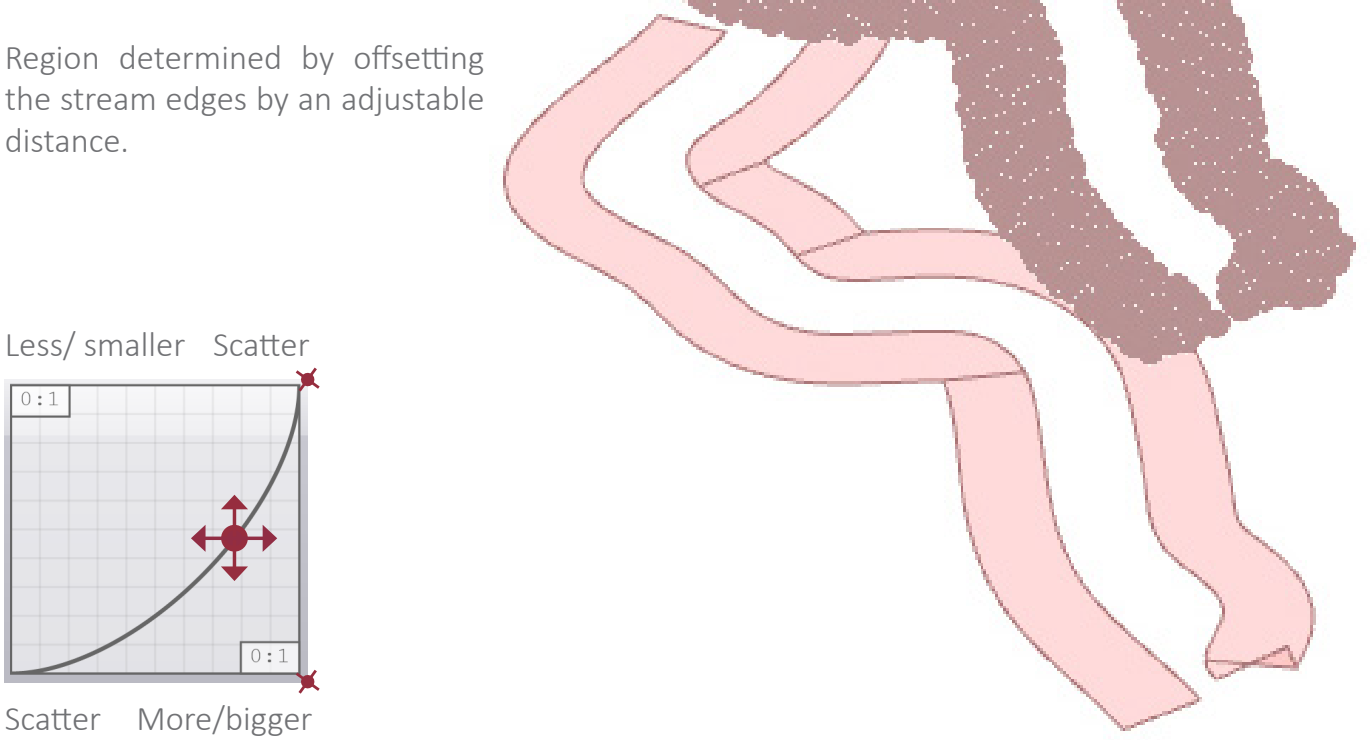

Figure.3.27. Graph mapper (conic graph) used to modify cull pattern to points in order to get polygons. Based upon Figure.3.28. Diagram distance from each point to the stream edge. of bank vegetation tool. 
Stream vegetation variations Variation 1
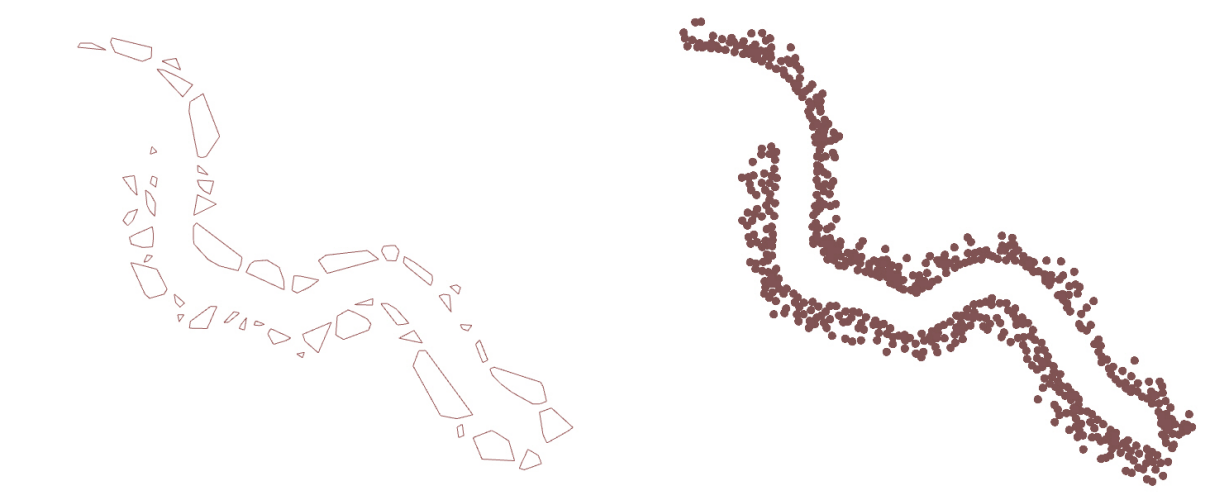

Variation 2

Variation 3
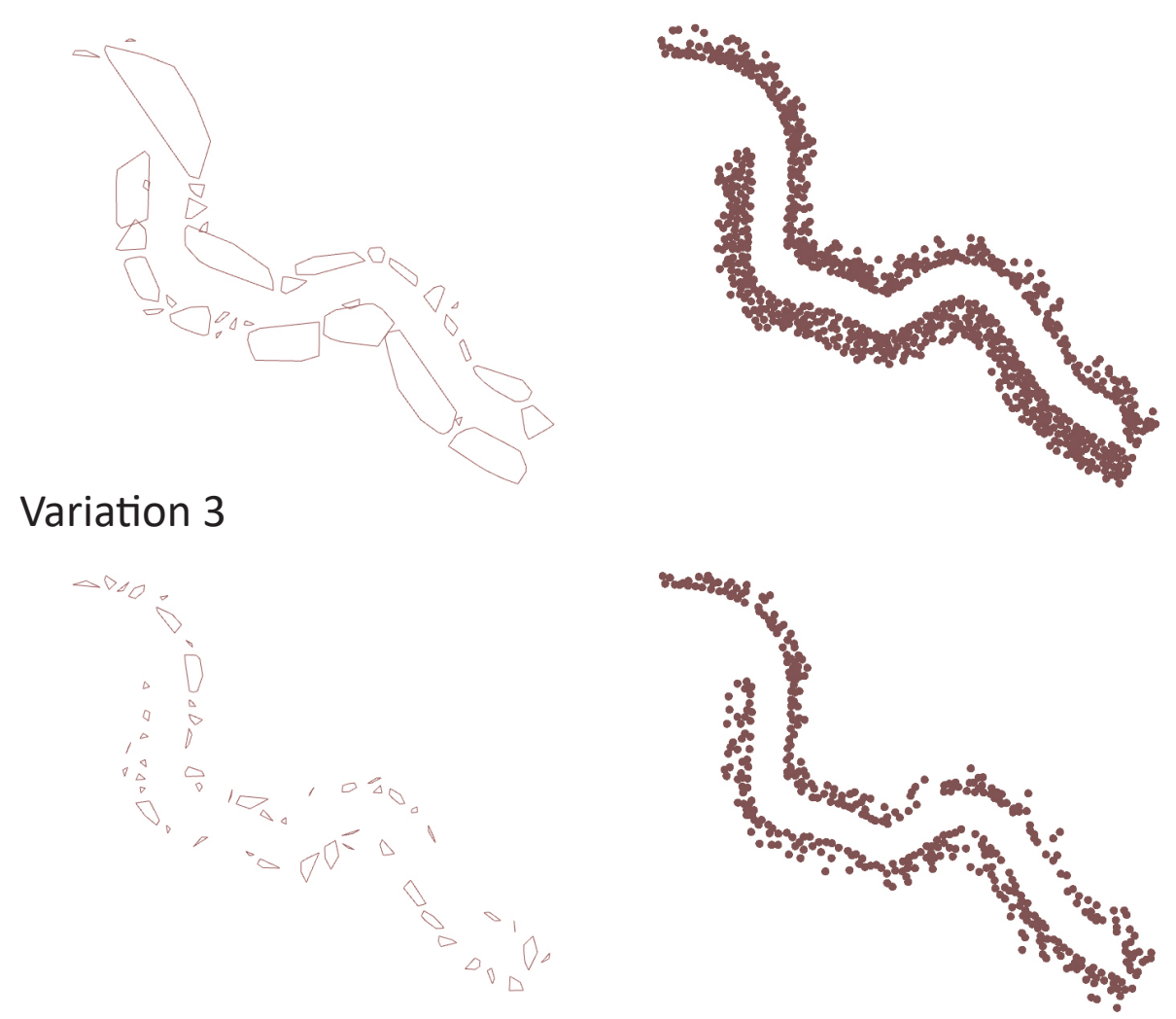

Variation 4
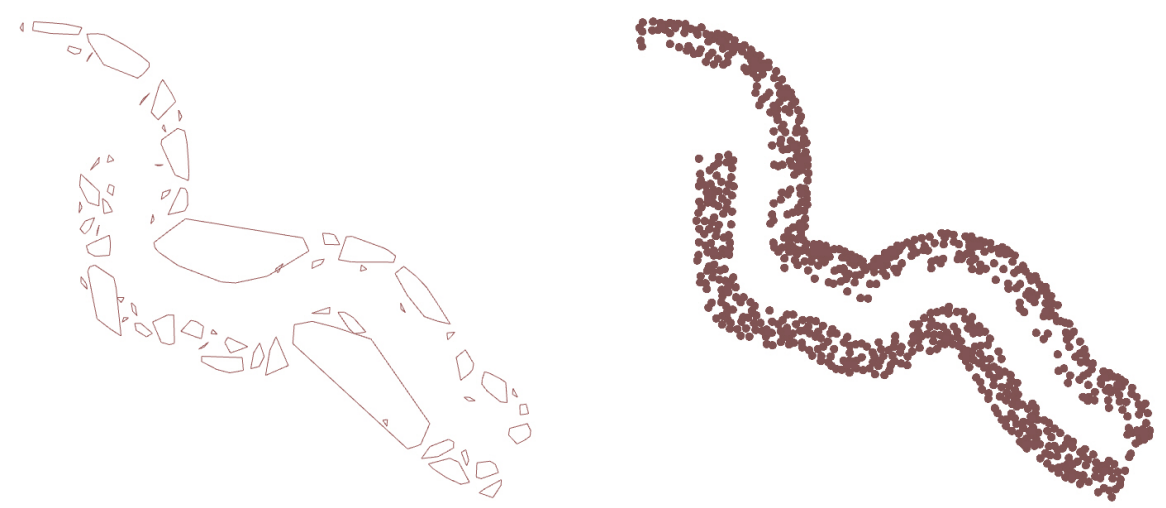

Figure.3.29. Diagram of bank vegetation tool. 


\section{Further Variations}

Further variations of both the stream path and vegetation clusters.

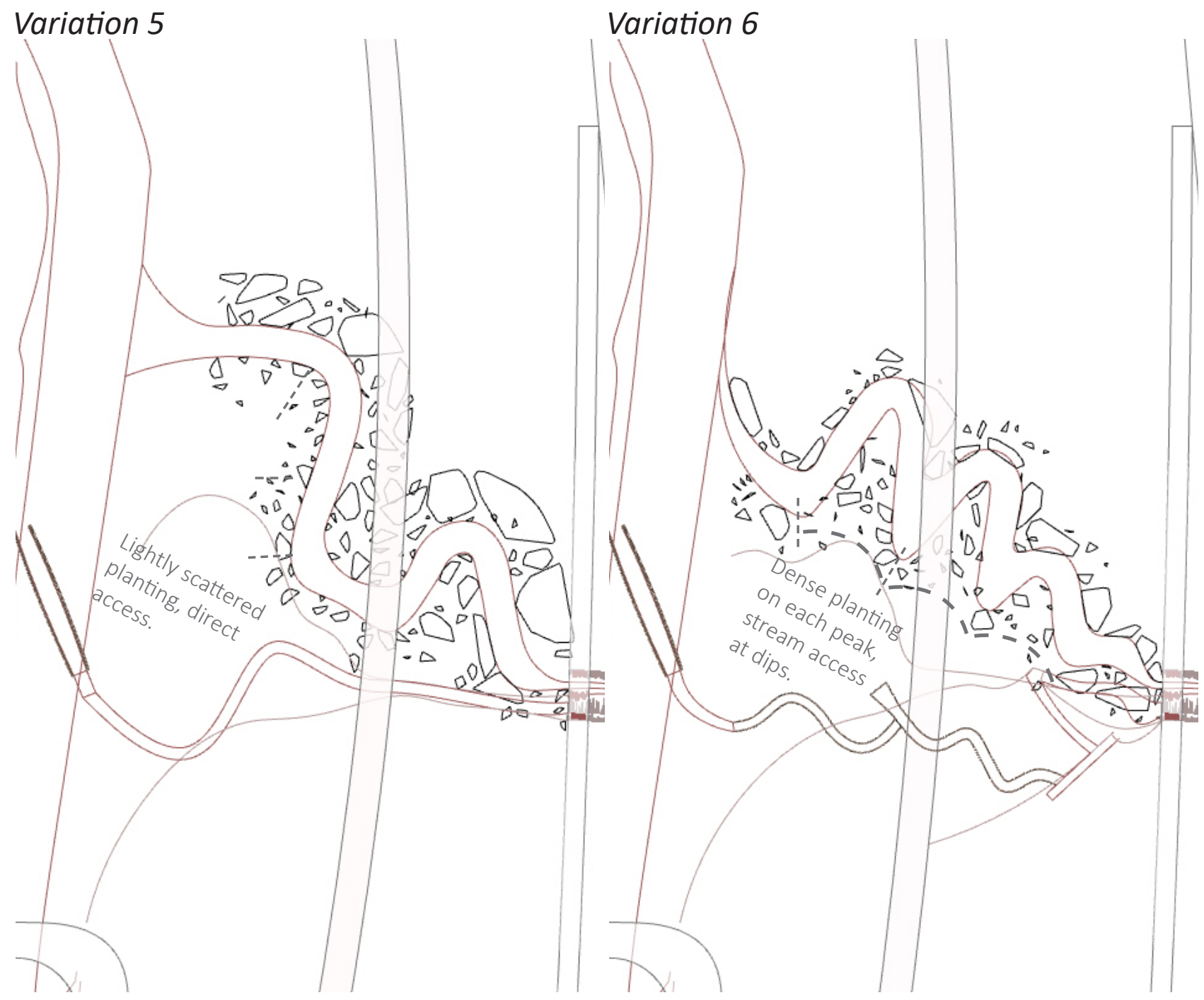

Figure.3.30. Variations of vegetation generation with stream generation. 


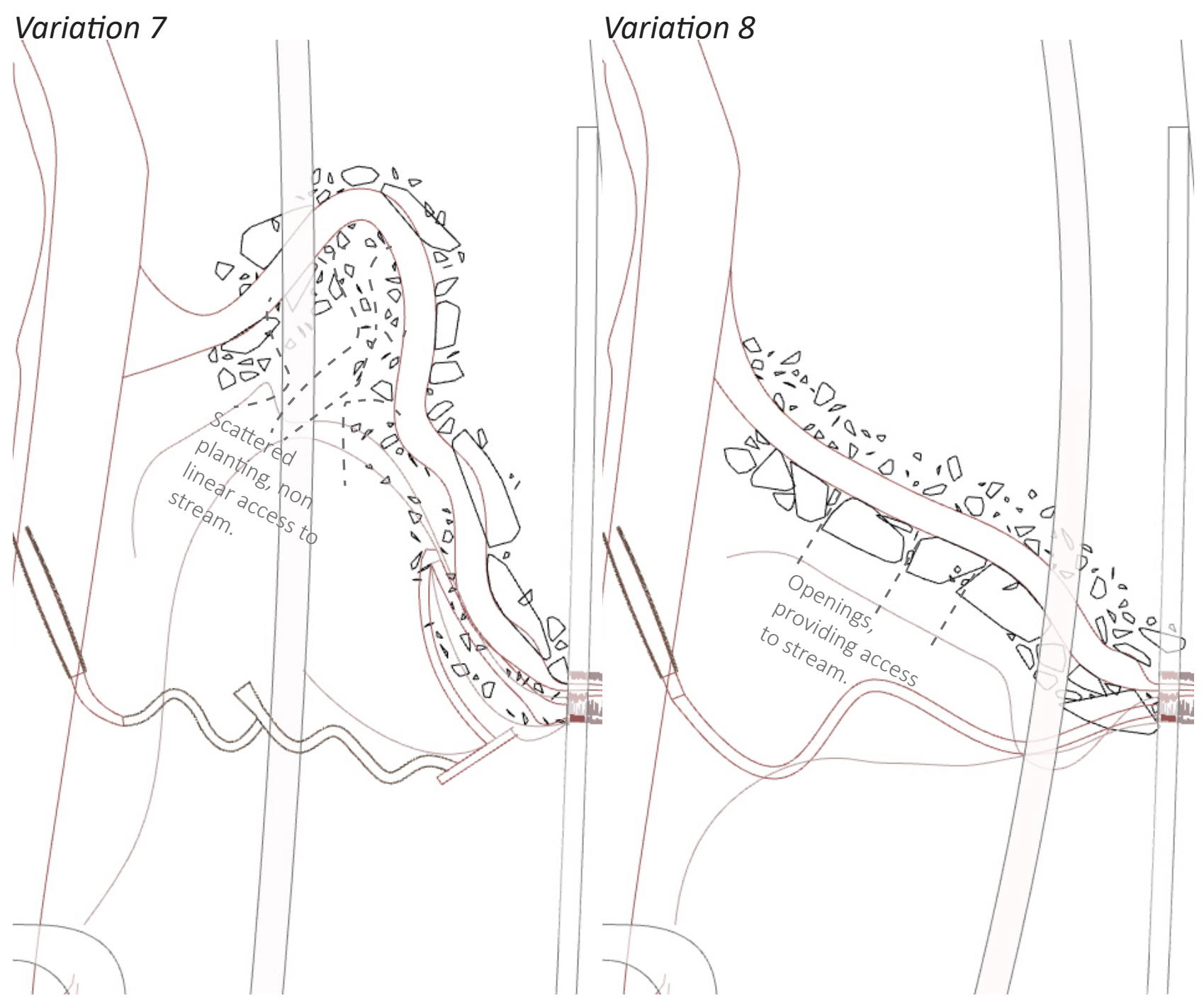

Figure.3.31. Further variations of vegetation generation with stream generation.

Page 116|Study 3 
Page intentionally left blank |Page 117 


\section{Interface}

The second study at Moa point augmented the interface to a high degree, creating a set of tools that accommodated a mode of pulling and pushing to figure out how a path might traverse the hills. Toolmaking was once again utilised in this study as means of sectioning the model for further perception. Capitalising on the dynamic nature of the Grasshopper and geometric relationships, the sectioning tool enabled a conventional exploration of the variations that were being generated. As the geometries are constantly related, updated to the model will update the sections. This additional tool helped to better understand the vegetation distribution and its relation to, topography and the pathways. The section tool had control over the angle and position of the cuts as well as the amount of cuts and the spacing between them.

Crude vegetation models generate at the intersection of the section cuts and the vegetation polygons.

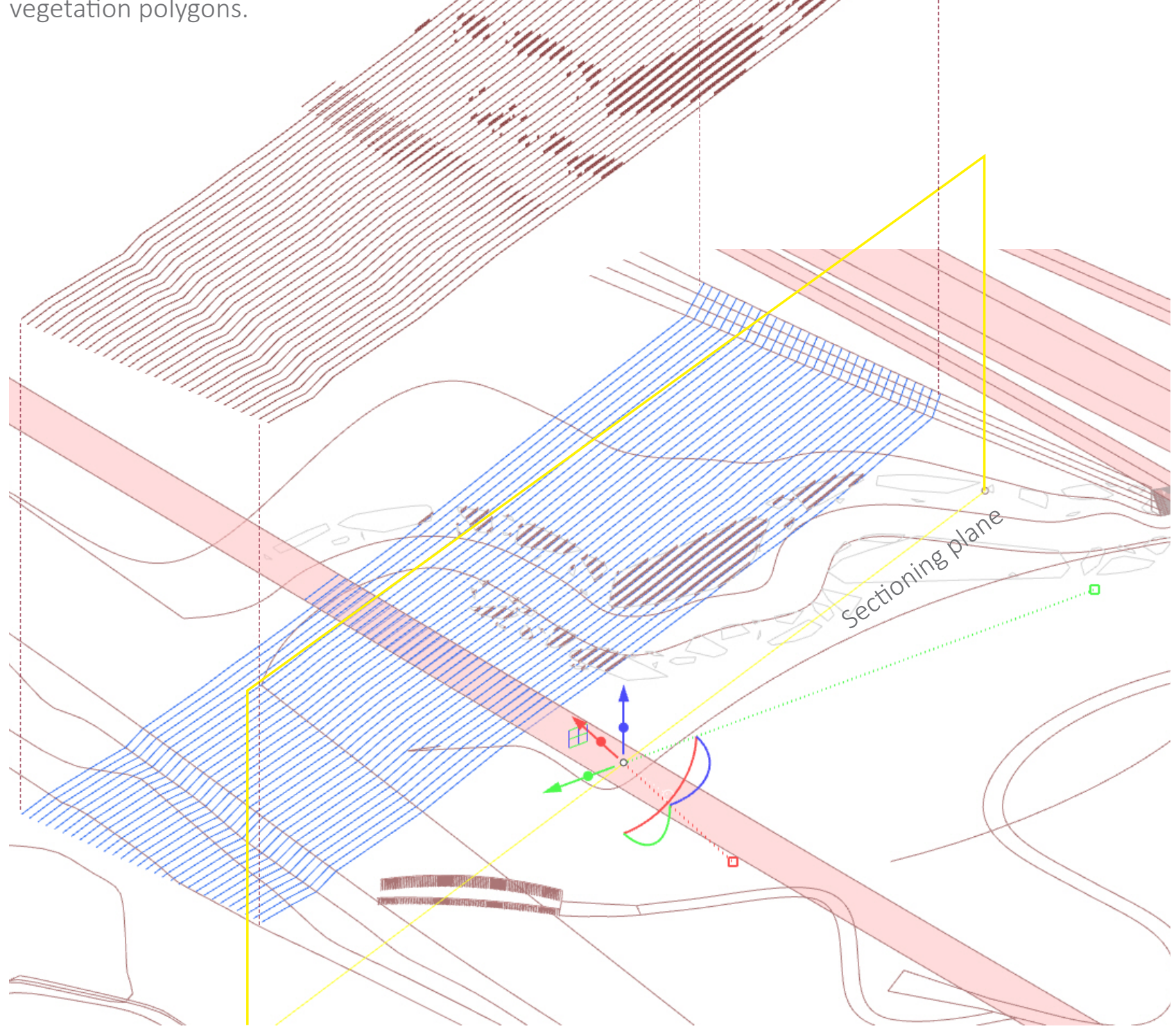

Figure.3.32. Sectioning tool.

Page 118| Study 3 


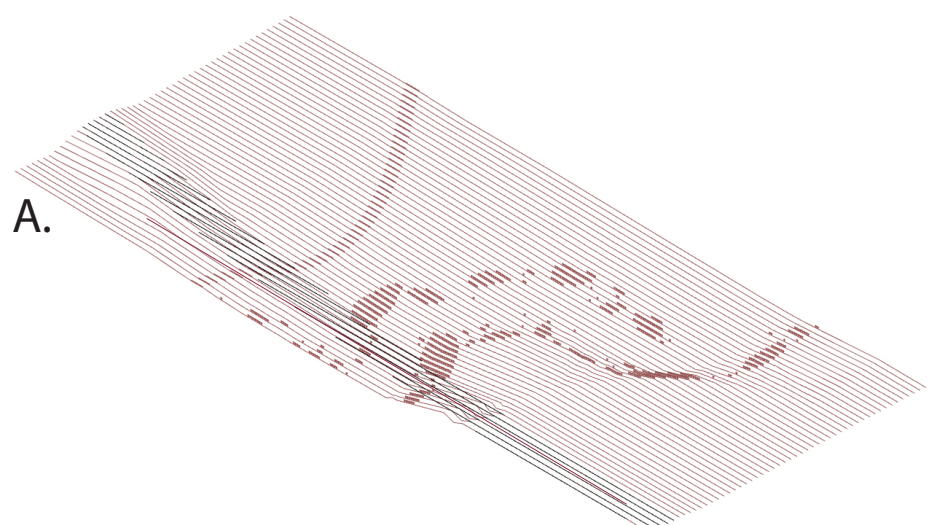

B.

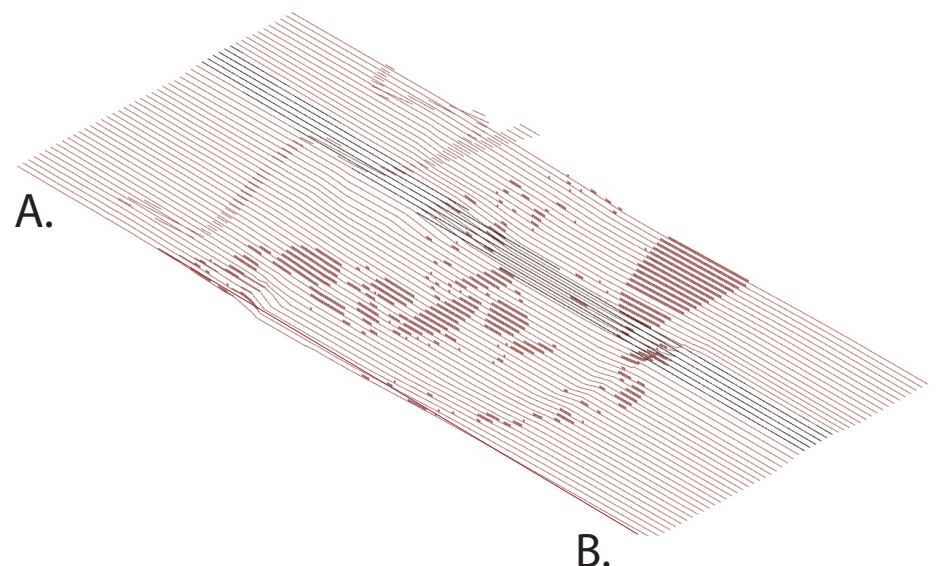

B.

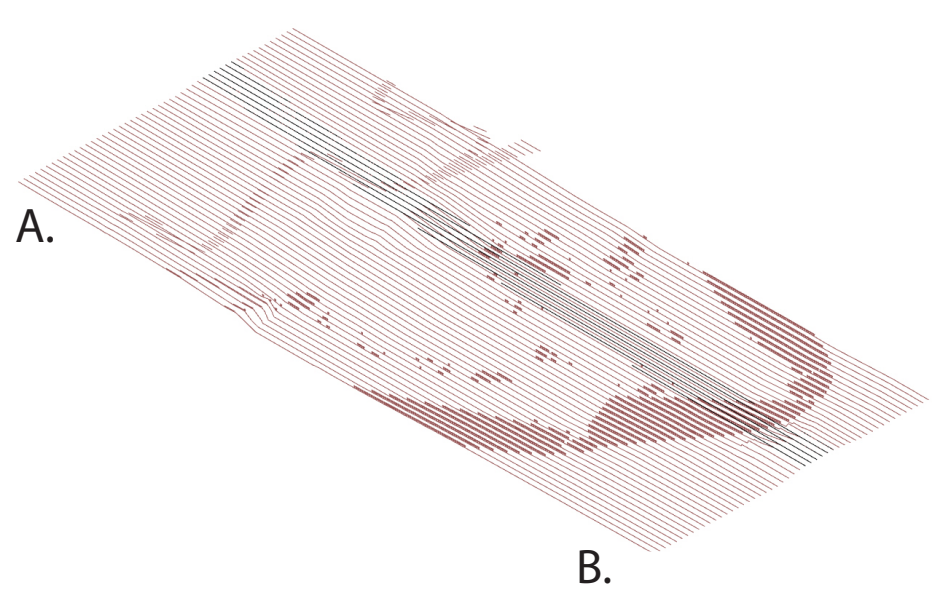

A.

A.

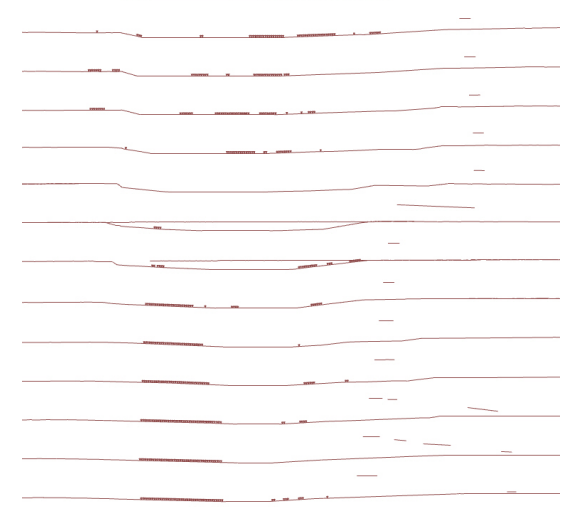

B.

A. $\quad$ B.

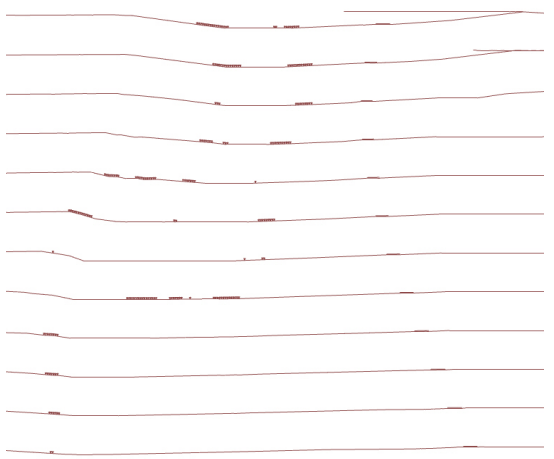




\section{Sectioning Tool}
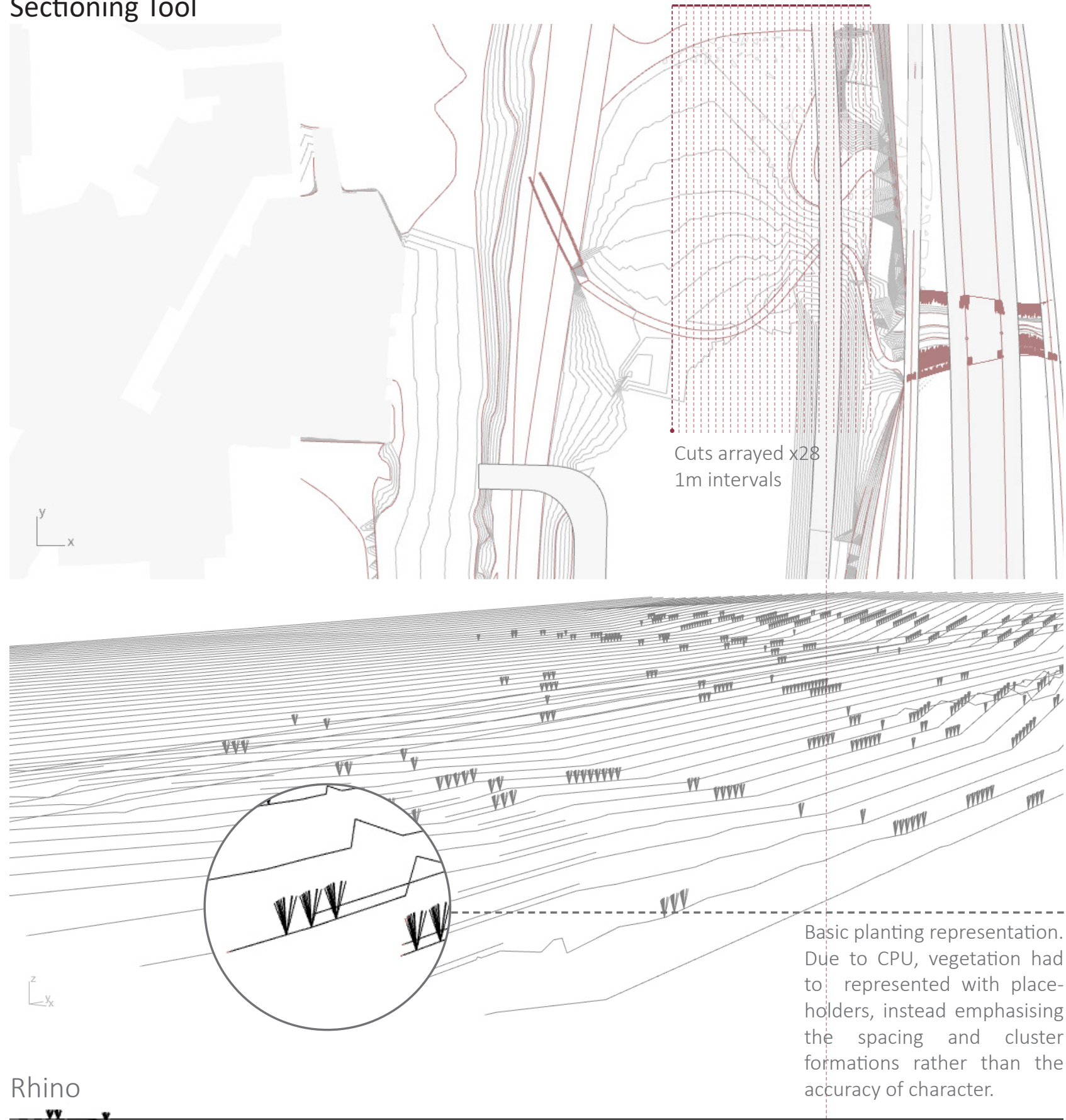

Rhino

vwrwwy

Reindered interpretations hellped to perceive potential textual qualities of the planting compositions.

Rendered

Figure.3.34. Further testing of sectioning tool. 

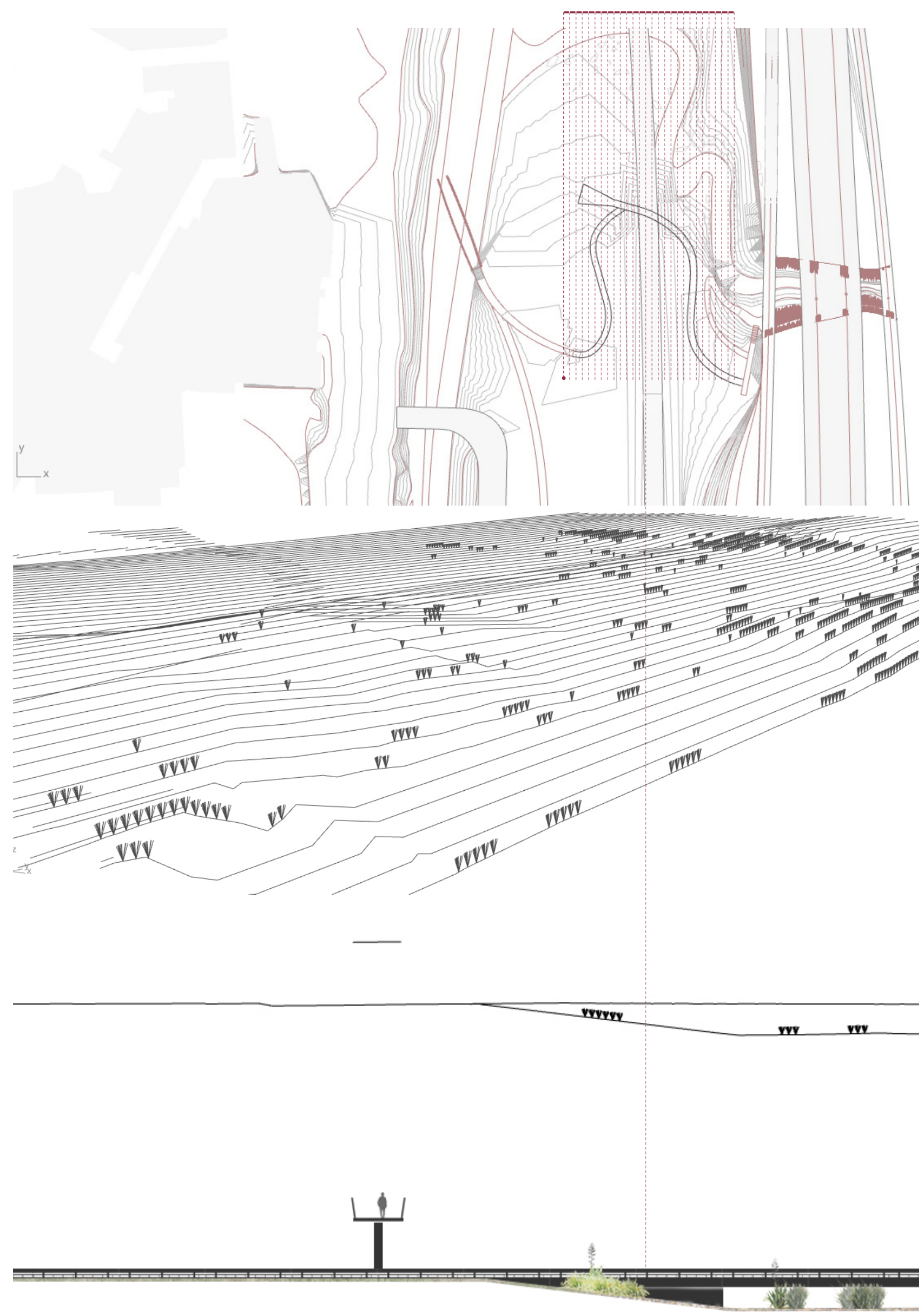

Figure.3.35. Further testing of sectioning tool. 
Testing variation at the same line.

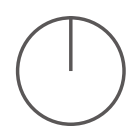

$0.25 \mathrm{~m}$ Contours
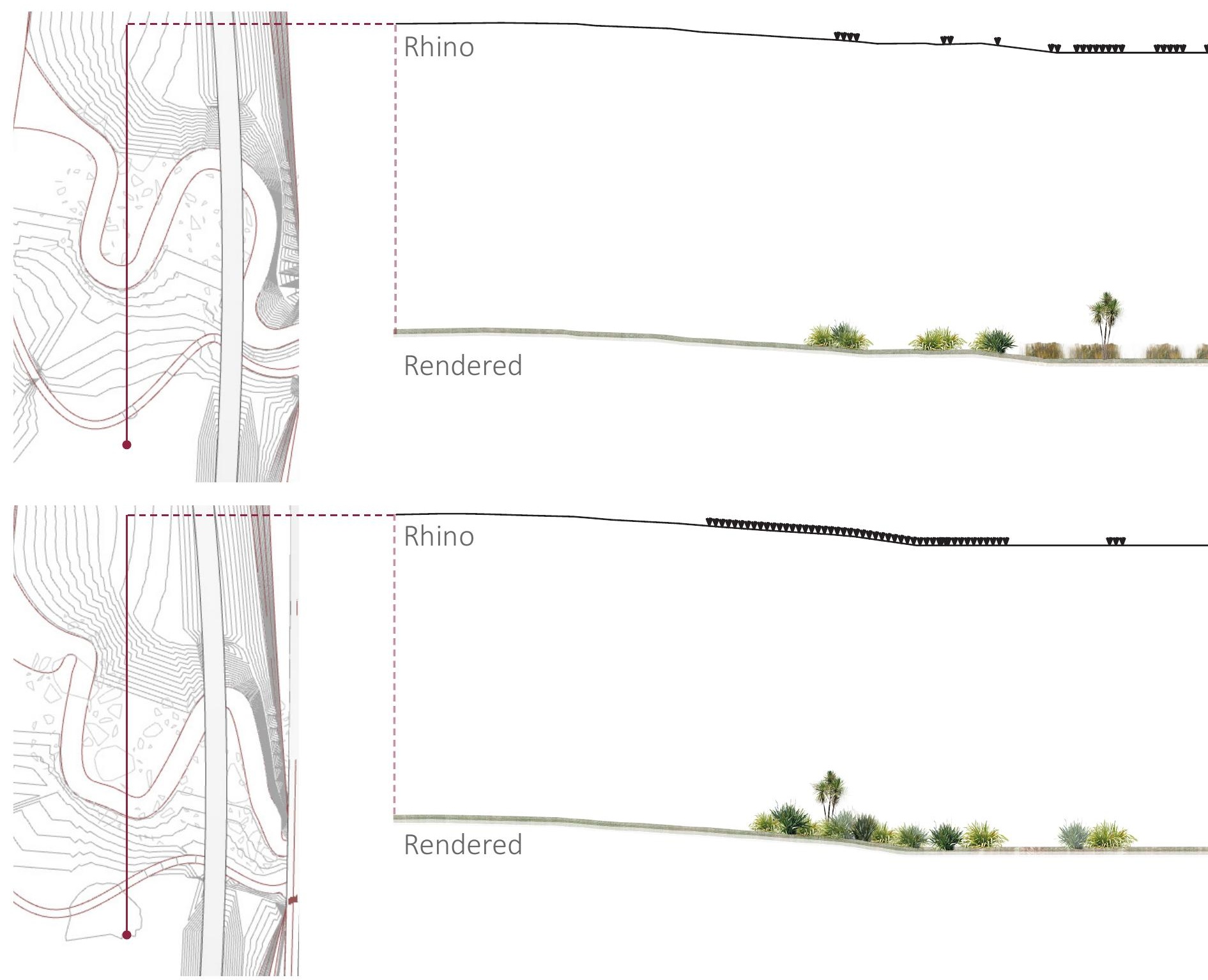

Rhino

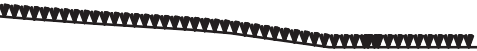

m

Rendered

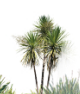

Figure.3.36. Variation test at the same cutting line.

Page 122| Study 3 


\section{Overpass and Slope Checking}

The overpass was adaptable to changes in the stream, topography and pathway. It's flexibility is made possible with point geometries acting as joints for the overpass to bend and shift in reference to. The slope could be analysed quickly to adapt to specific slope requirements, such as under \%8 which is required for Wheelchair access. (NZ Transport Agency, 2007)

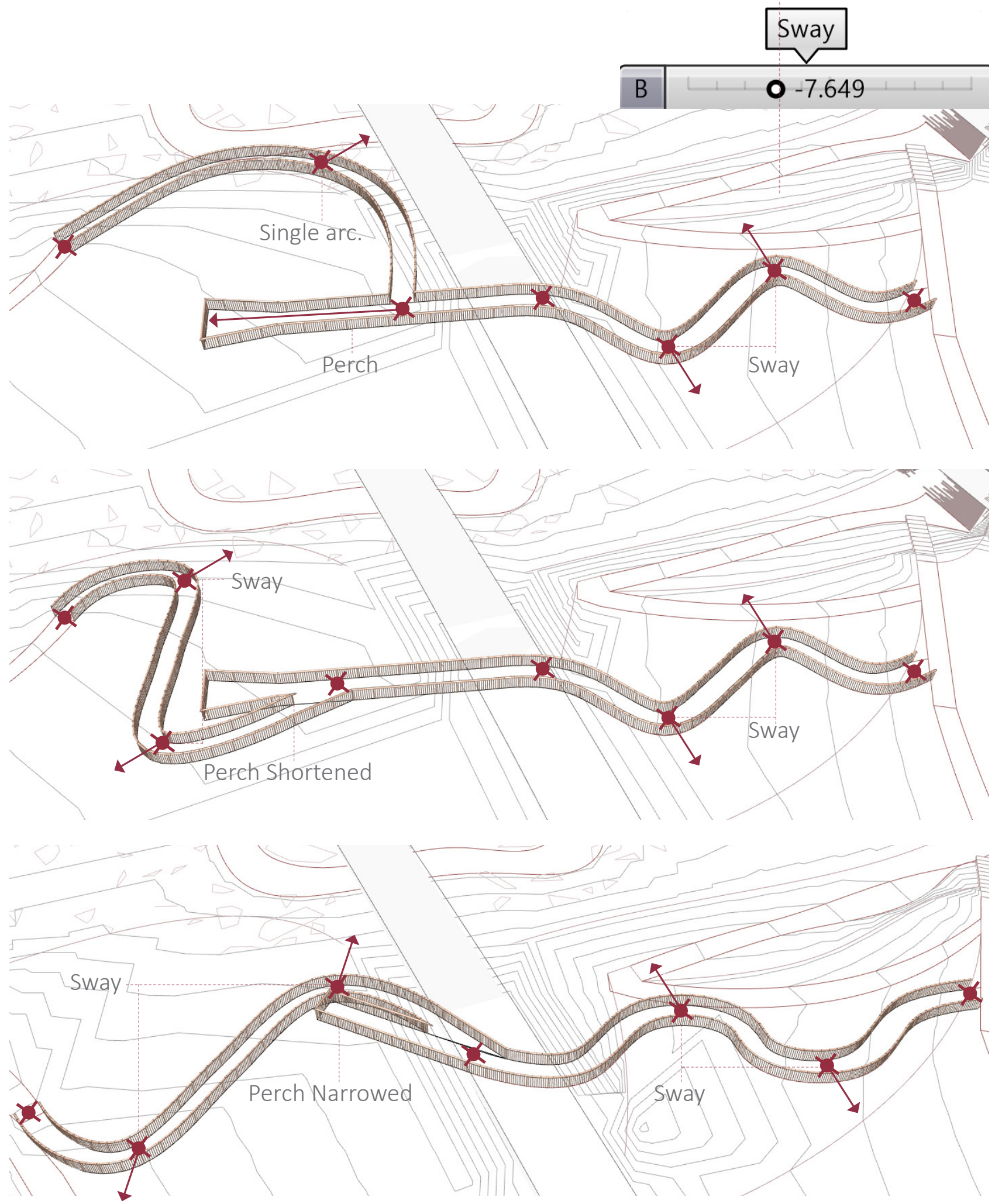

Figure.3.37. Overpass logic.

Page 124|Study 3 


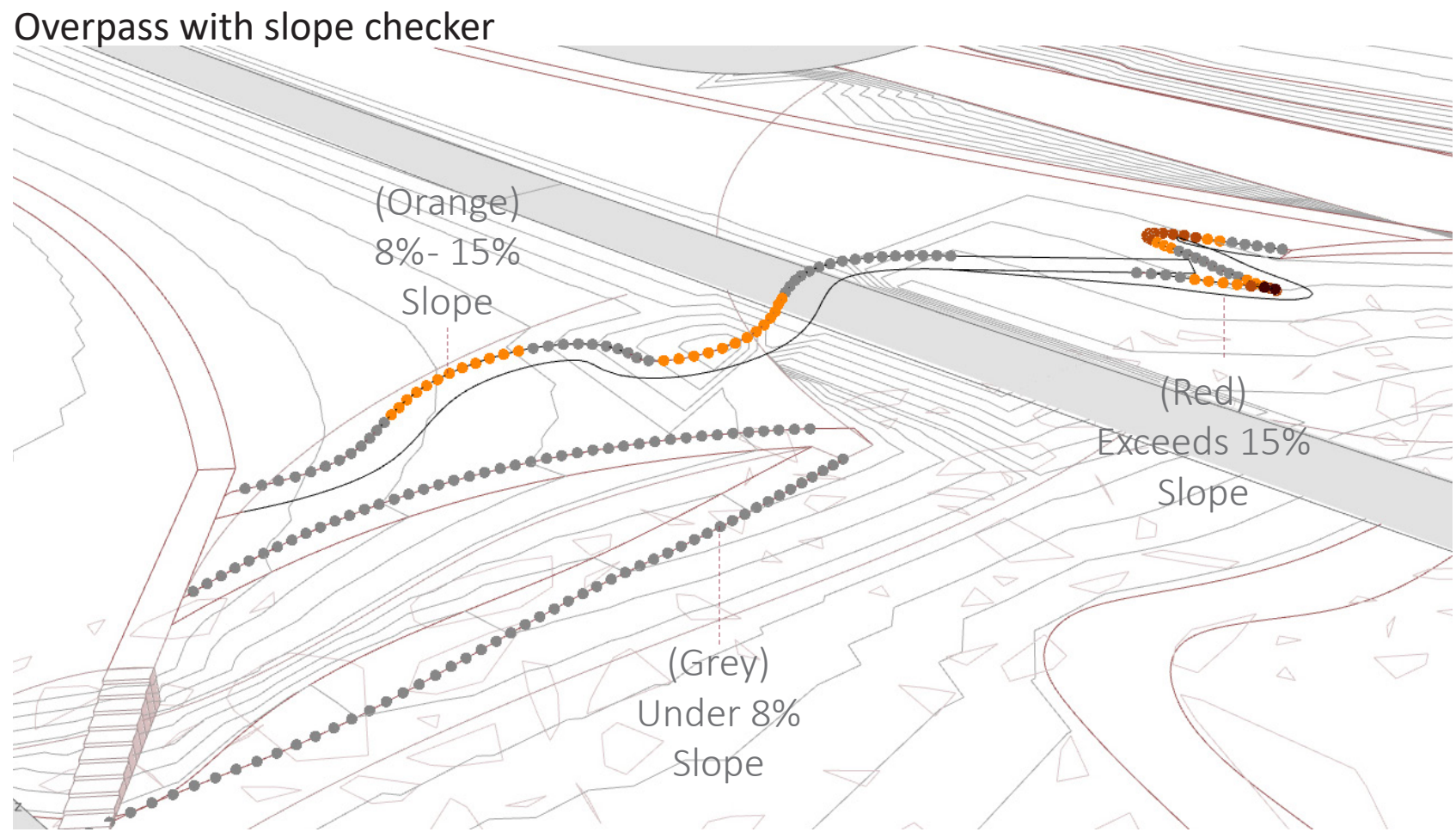

Overpass all under $\% 8$

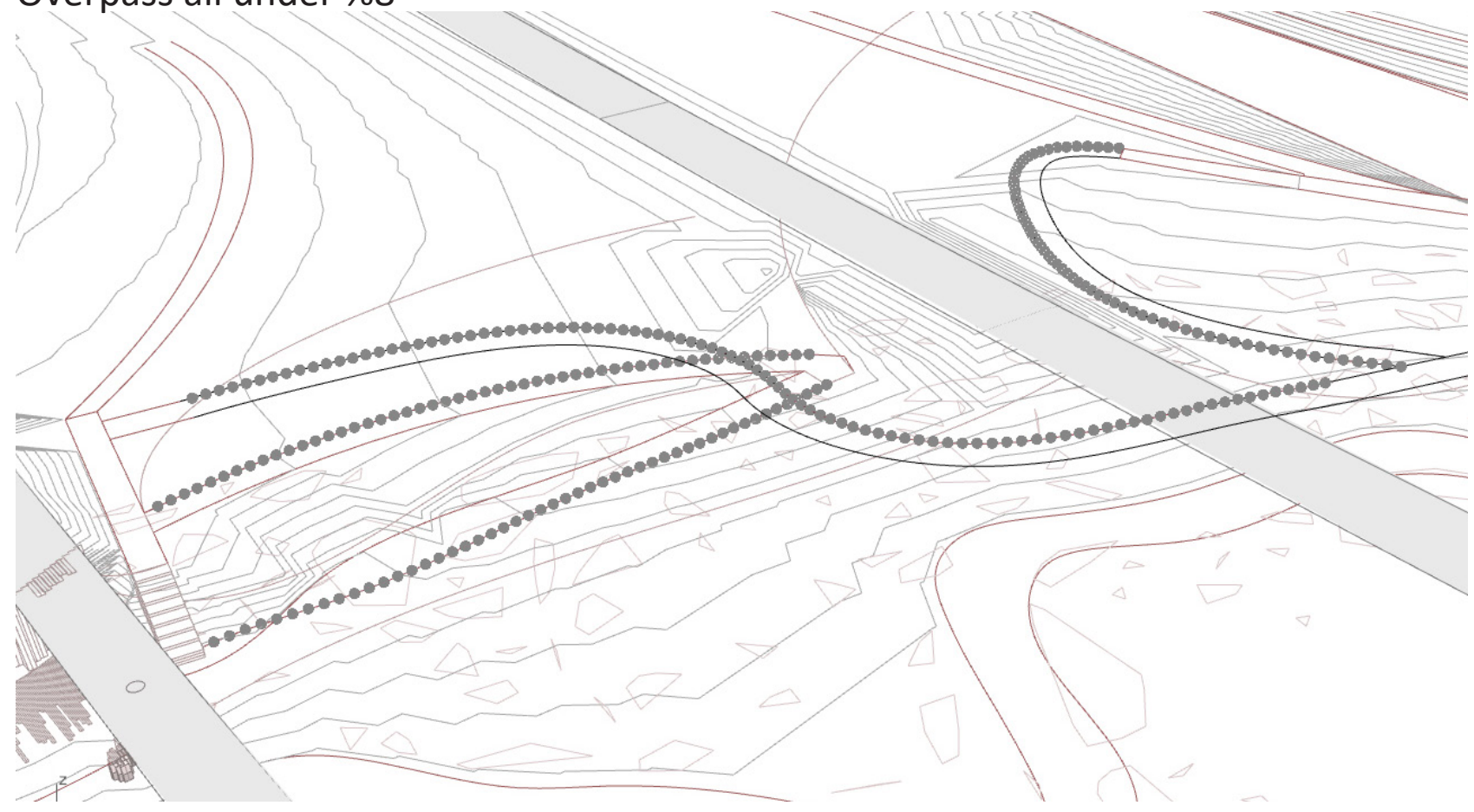

Figure.3.38. Slope checking tool. 


\section{Script and Management}

Something that this third study could have improved upon as is evident by the spiders' weblike mess that can be seen in the in the background of Fig.3.39. The script structure is ordered to areas by purpose and naturally grew over time from top left to bottom right. Each area had a controller to modify the components for each element. As complexity grew the model tended to be progressively more difficult to manage as new areas of the script appeared. Subsequent programming of parameters had to be carefully managed as to not impede on the programming of other elements, if so the script might not function as intended. A script this large becomes vulnerable to errors and the accidental removal of one logic may collapse the entire structure, requiring tedious fixes. However, part of the scripting process is to have a good managing system and to have foresight as to the direction the script is growing. This is something learnt through failure yet may turn off many designers using algorithmic tools for large scale dependability such as this in practice.

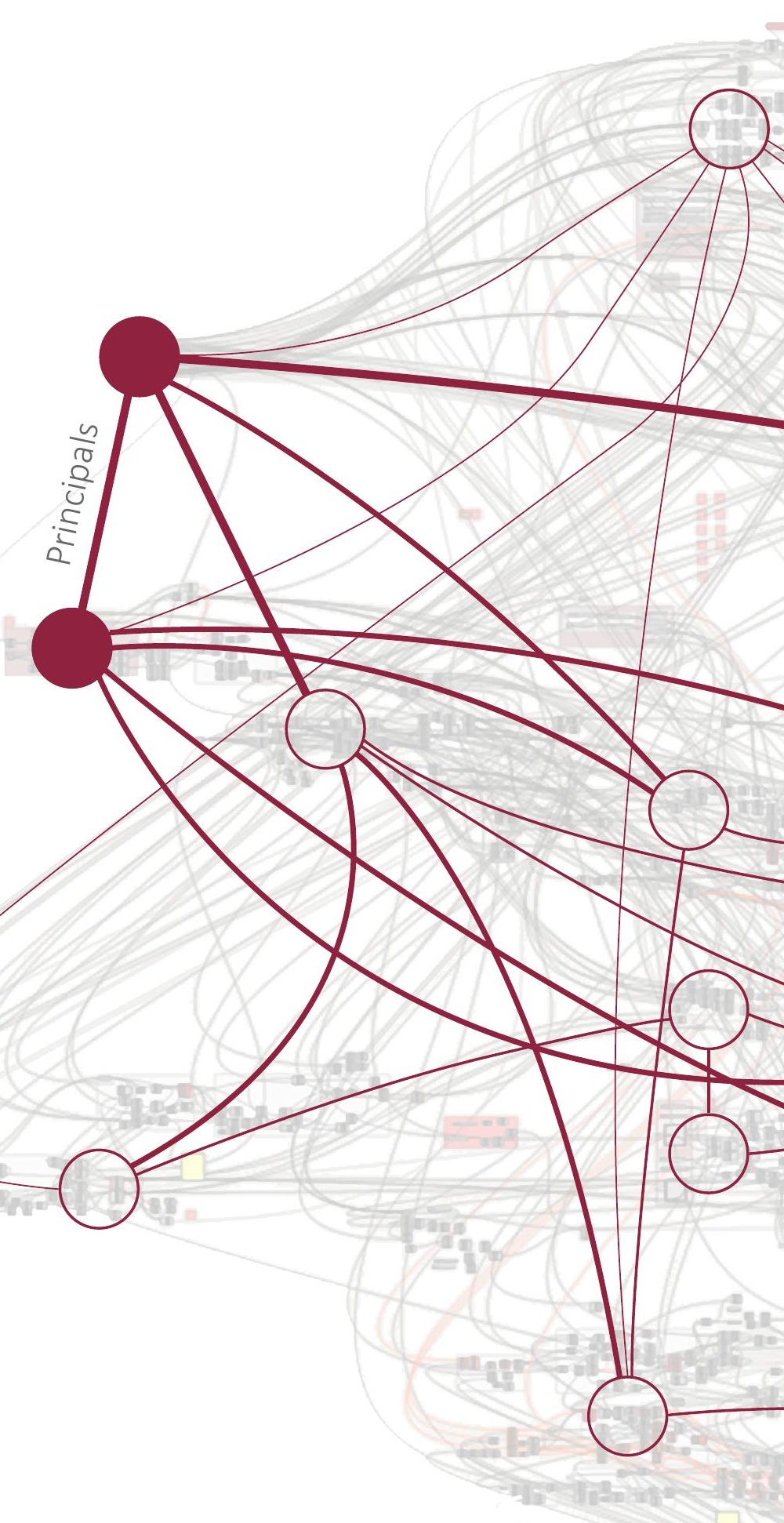




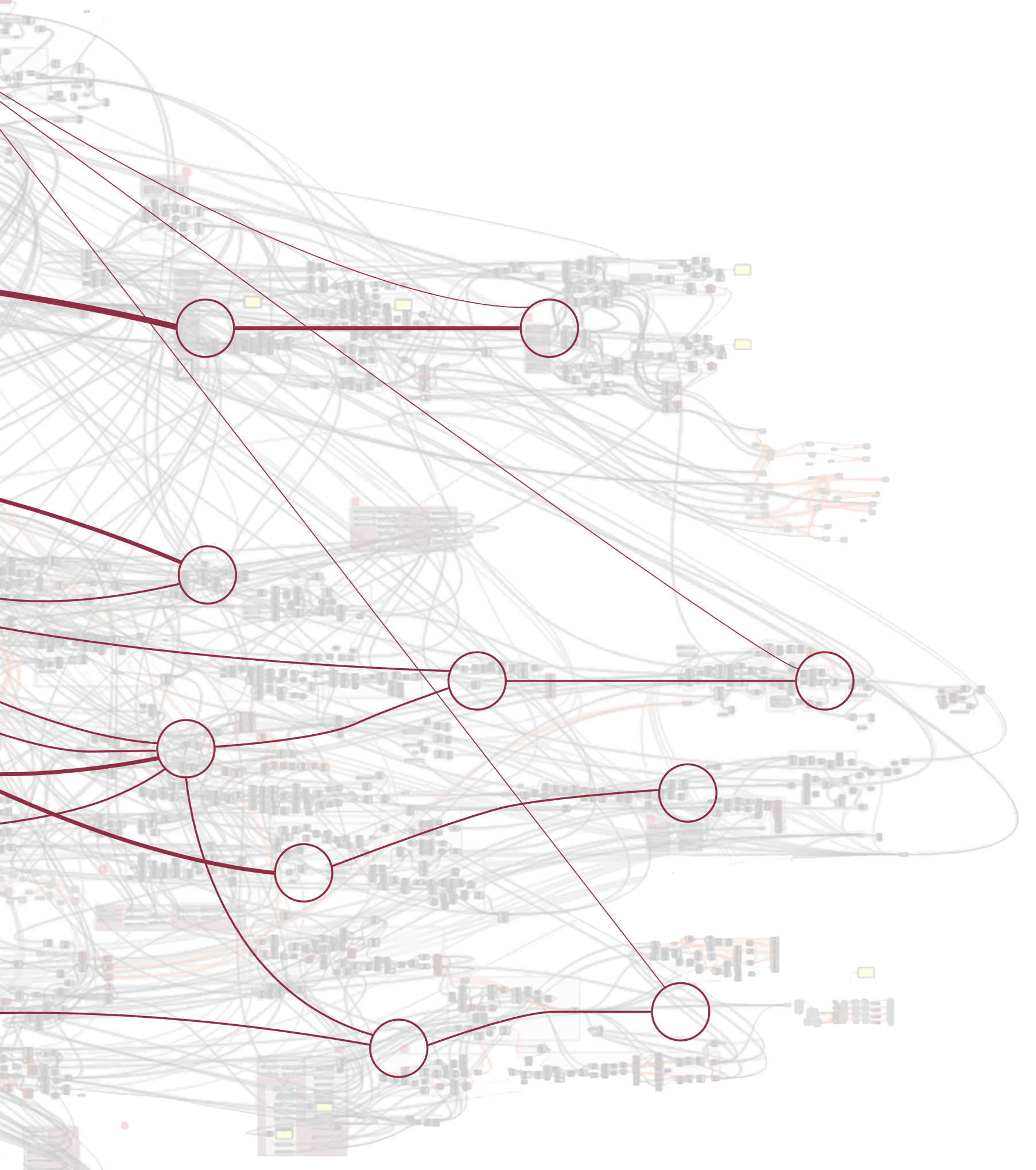




\section{Diagram of Script}

To translate the script (fig.3.39) this flow chart represents how each logic is dependent on one another. To comment how better to manage a large script, planning through diagrams such as this may prove useful to guide the growth of the script. Through finding principals, logics can be planned sequentially as to their relation to the principal and to one another.

\section{Interchange}

The Interchange and rail were the dominant site variables, as the movement of these two variables was the primary investigation they became the leading logics/ principals.

\section{— Underpass}

The form of the underpass was directly related to the shifting of the interchange, not effected by the rail.

Rail 
The path and stream begin at the end of the underpass. The ramp only occurs if overpass is active.
The path is defined by the contours. The contours are defined by the stream and Rail.
The mesh is generated from most elements. A point cloud is firstly made from previous logics then a mesh is formed using Grasshoppers' "Delaunay mesh" component.

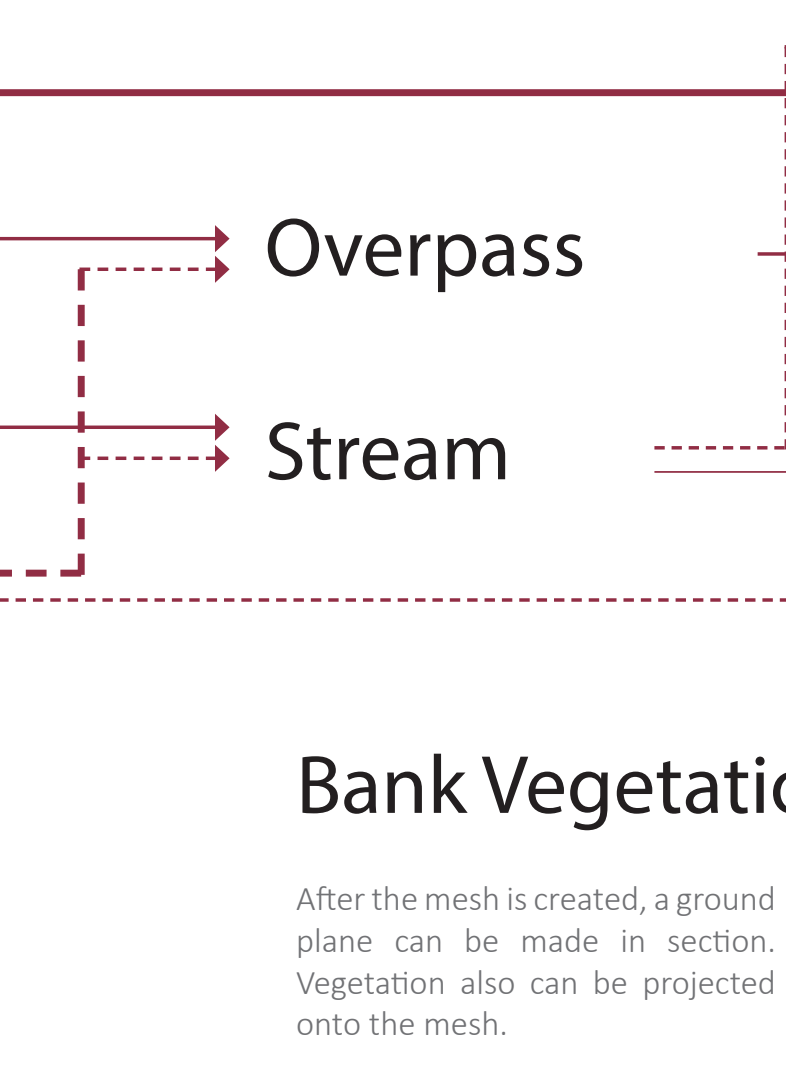

$\rightarrow$ Contours

$\rightarrow$ Ramp

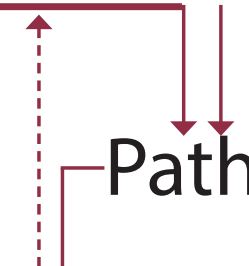

Mesh

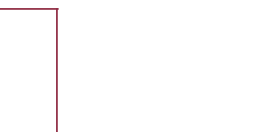

$\downarrow$
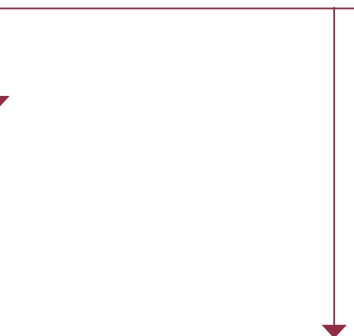

Section Tool 


\section{Reference Geometry}

Within this model, at least $\% 70$ of the elements were hidden. The hidden geometries were to project, push, pull and parametrise the \%30 revealed elements that make up the seen model, existing purely for reference and guiding. The dynamic nature of the model is made possible by these hidden geometries, functioning like clockwork.

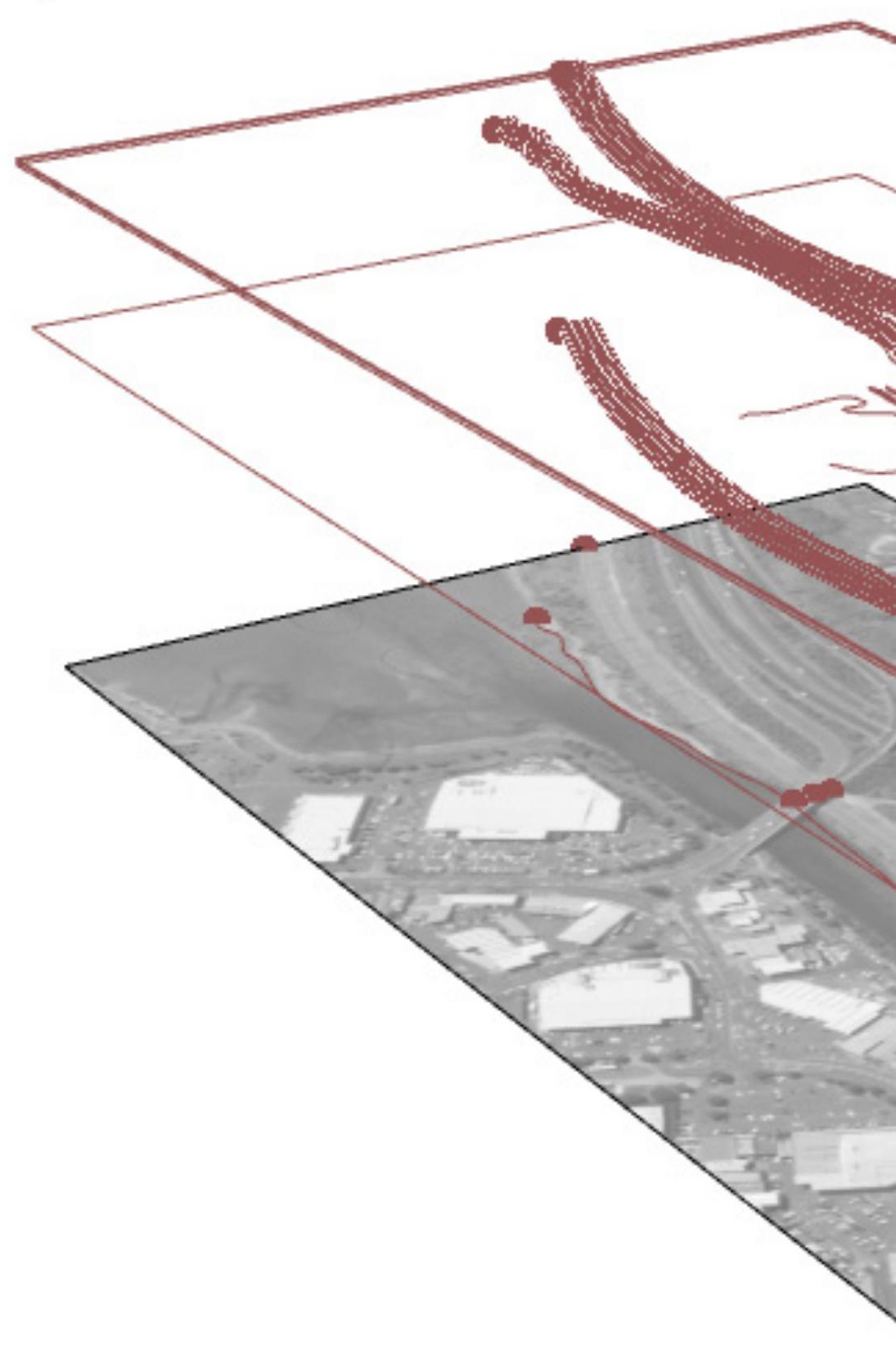




\section{Tool}

The tool operates as a dynamic model built upon a set of designed parameters by which each geometry can move accordingly. By shifting values with sliders the design can morph into a new concept, based on the parameters set by the designer. The parameters then, decide the margin for all potential outcome therefore under refinement of the designer they embody all design bias and intention. The designer knows to some level, the outputs that will occur based on the values of the input. The ability to predict outcome is related to the complexity of the script and the allowance for random generation (such as how the stream generated itself). The potential design variations for this study is far larger than that of the first study in Tory Street. As this final study took on a non-linear site and adopted a far larger and more complex script therefore having more potential outcomes. The variations by style are similar yet compositionally they can vary profusely, most notably in the topography. The tool recomposes the variables into new concepts, mediated by changing input values and shifting sliders, predicated on the common goal of connecting a path from the CBD to Cannons Creek.

\section{Model Parameters and relationships}

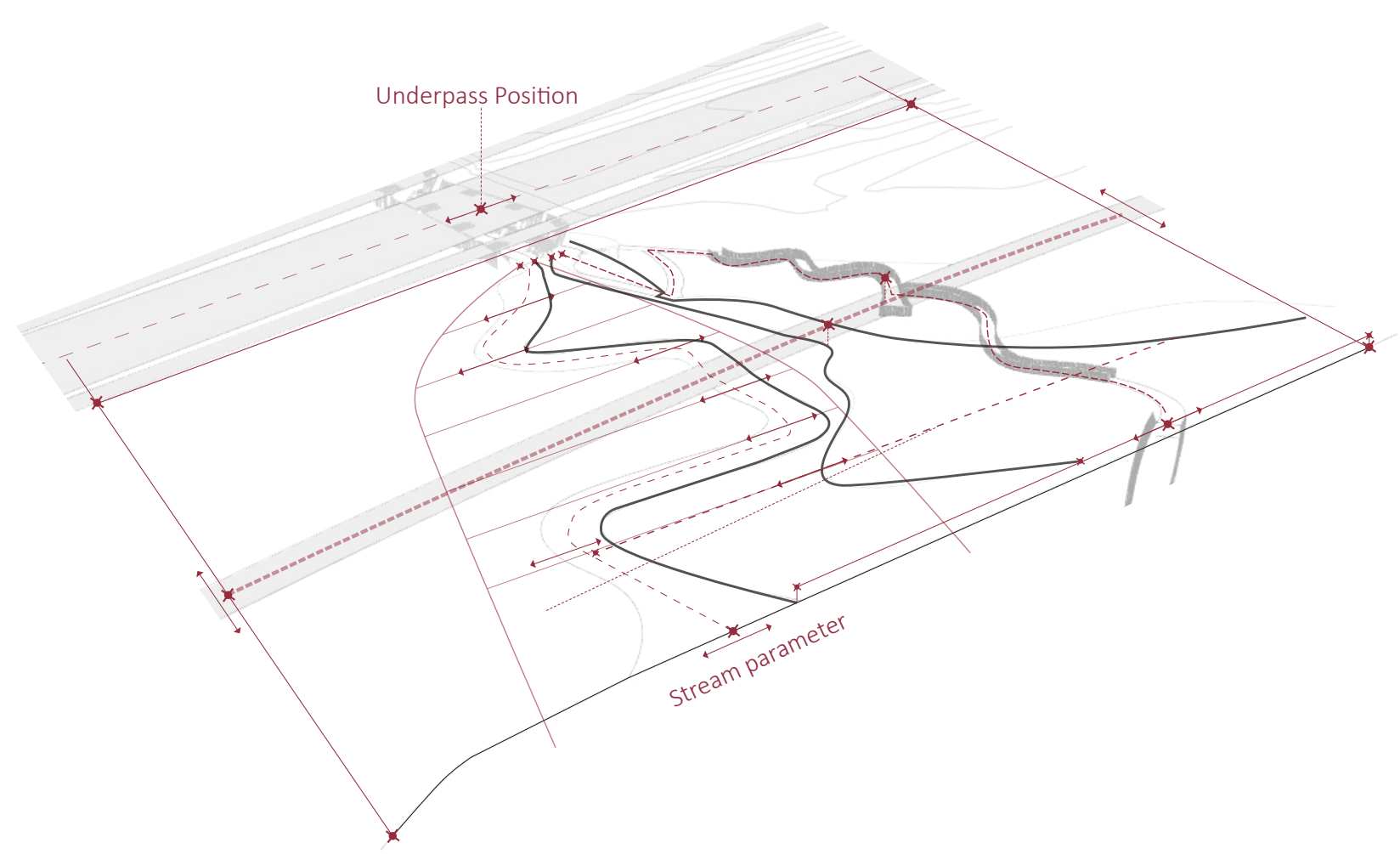

Figure.3.42. Parameter diagram

"In parametric modelling the importance of composition or geometries is replaced by the declaration of specific parameters or rules which become foundational to design outcome. This design approach shifts the focus from form as outcome to processes of formation, emphasising relationships over composition." (Wallis \& Rahman, 2016) 

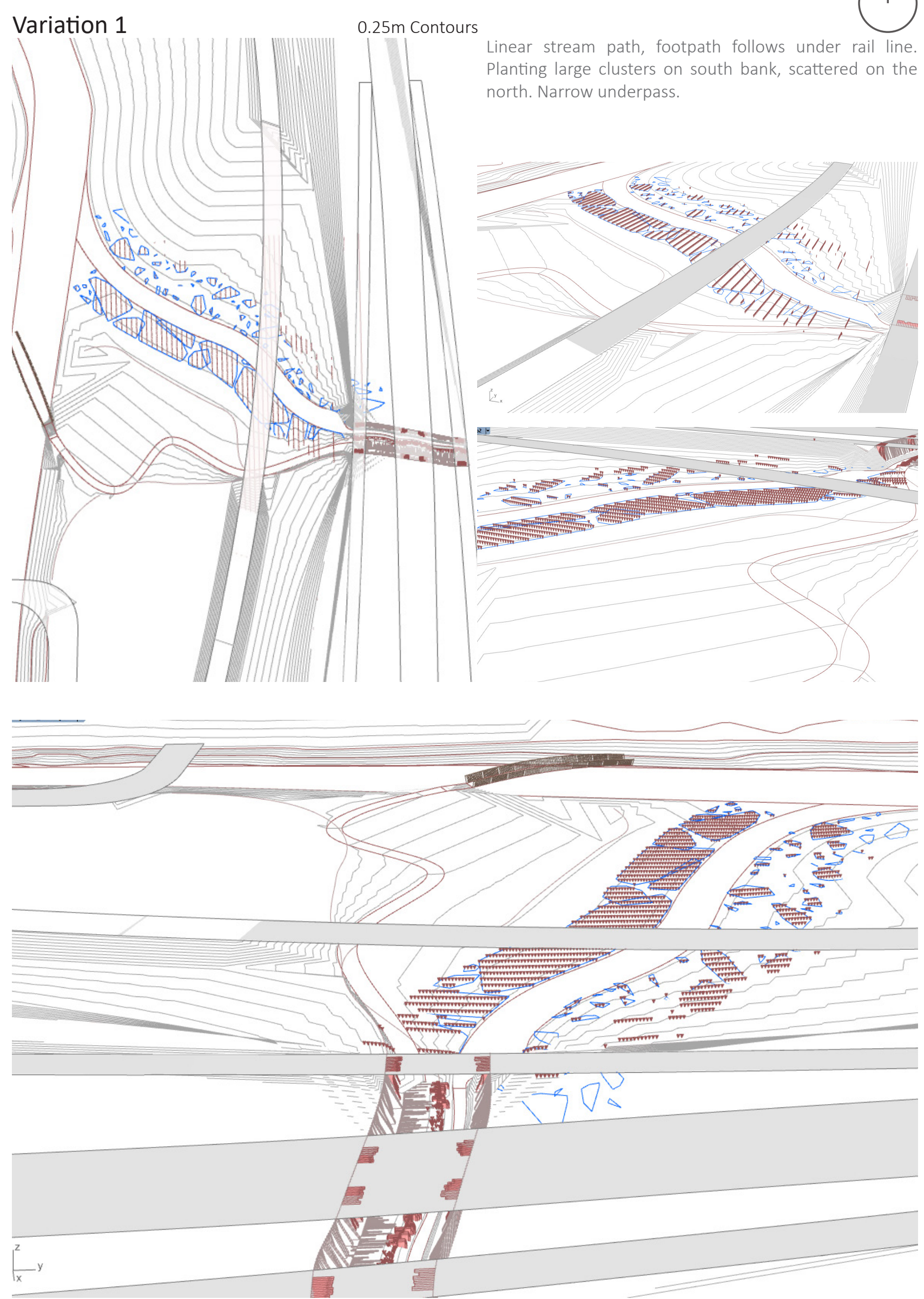

Figure.3.43. Variation test 1 of the script and its tools. 


\section{Variation 2}

1

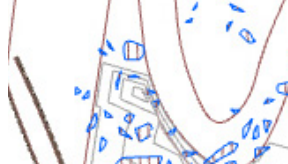

$1.0 \%$

Vor

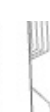

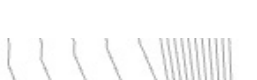

$0.25 \mathrm{~m}$ Contours

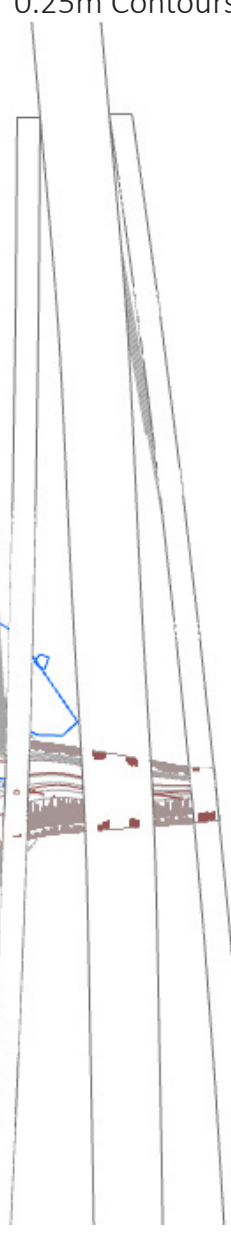

Highly varied stream path, widely scattered vegetation clusters. Lengthy overpass, underpass opening into a fan on the $\mathrm{CBD}$ side.
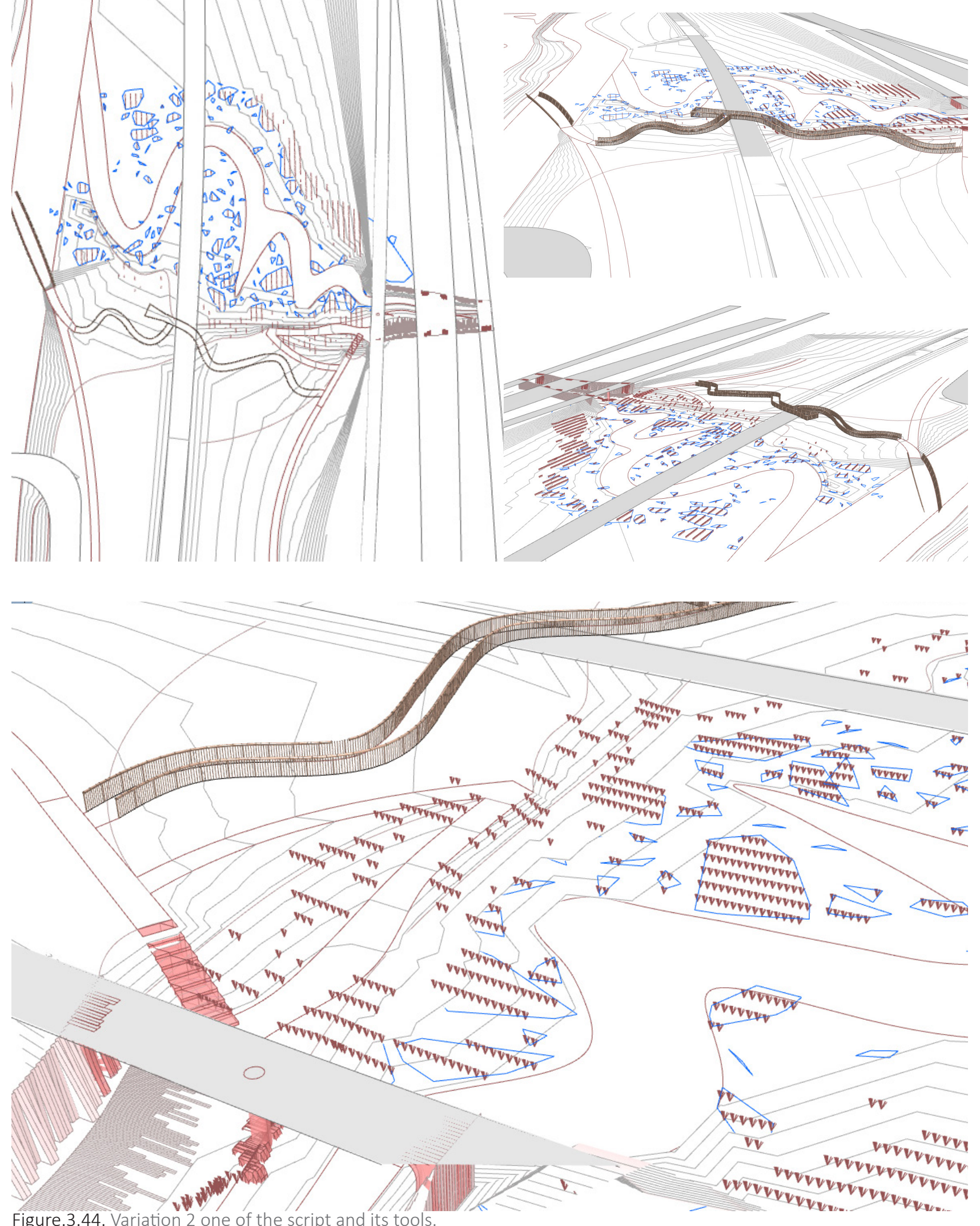

Figure.3.44. Variation 2 one of the script and its tools. 


\section{Variation 3}

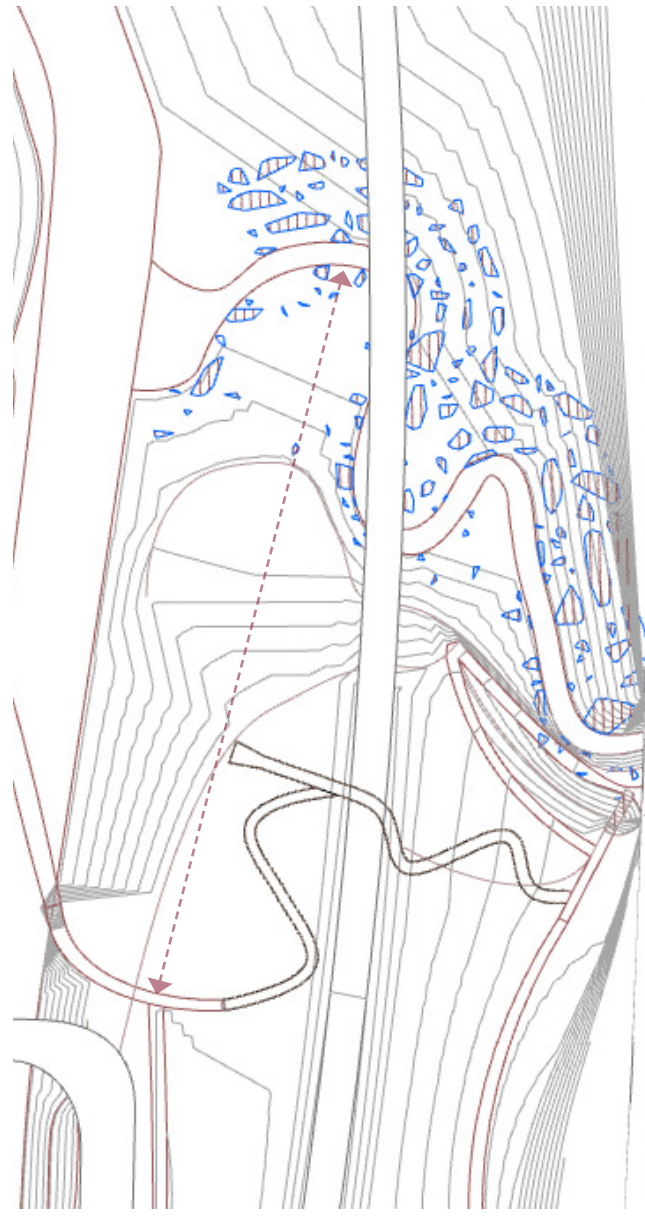

$0.25 \mathrm{~m}$ Contours

Overpass pulled back from stream edge, large amount of open space from path to stream. Vegetation sparse on the south side, dense on the north. Underpass widened.
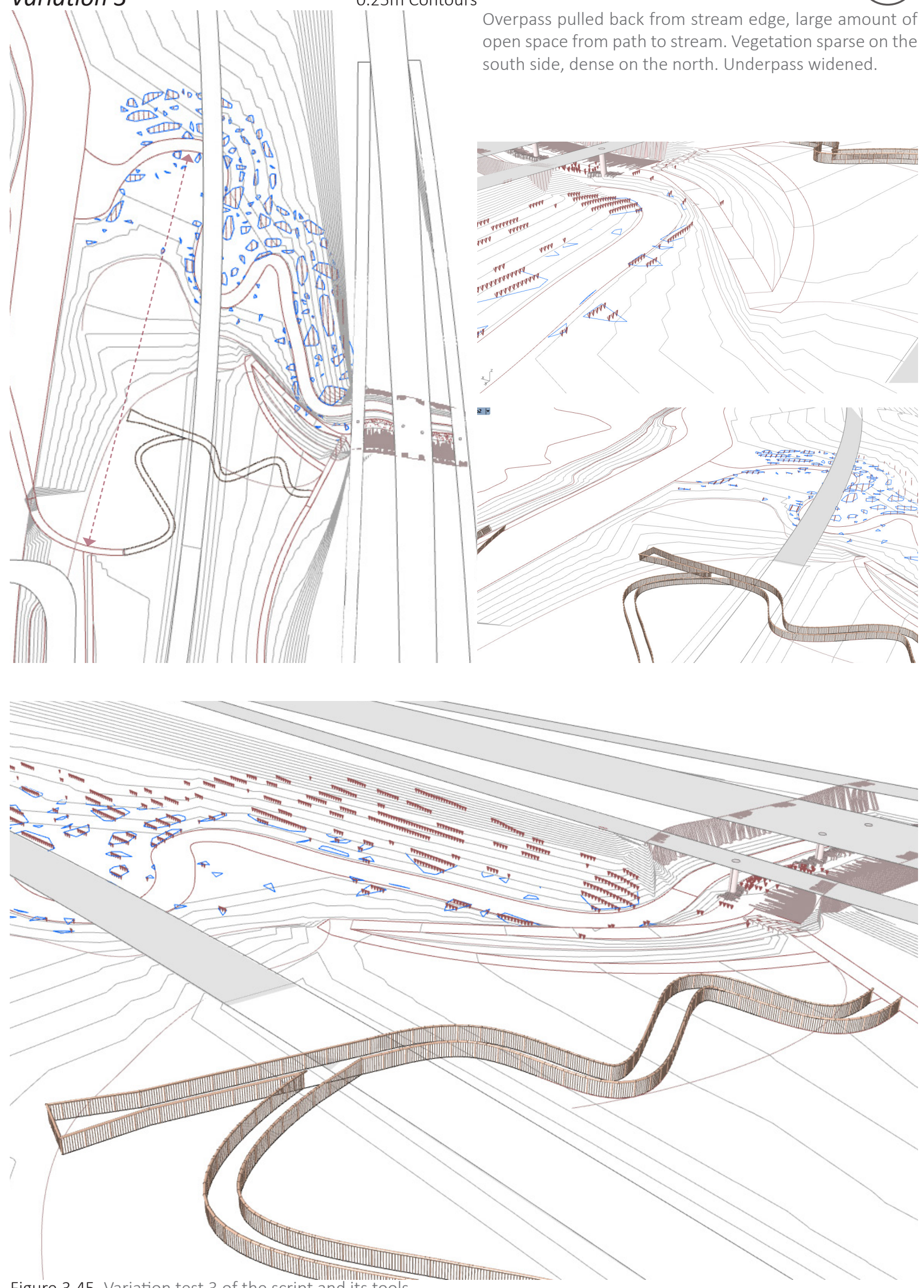

Figure.3.45. Variation test 3 of the script and its tools. 


\section{Variation 4}

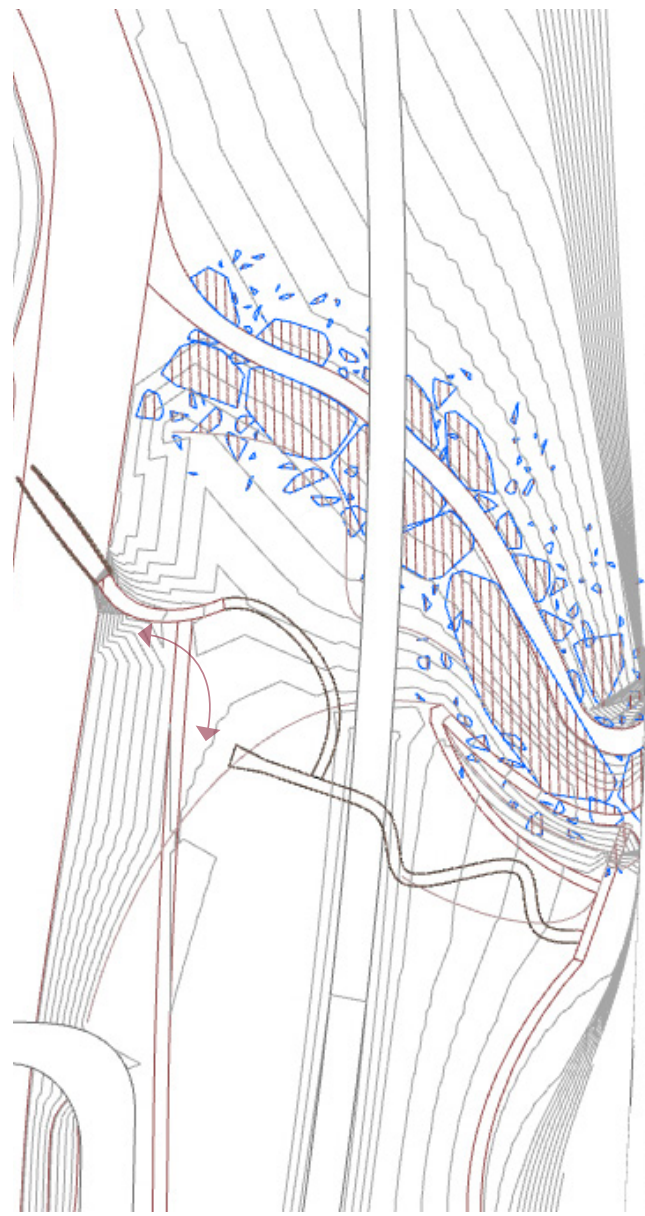

$0.25 \mathrm{~m}$ Contours

Large vegetation clusters, overpass arced on the left off-ramp as opposed to the wiggle pattern.
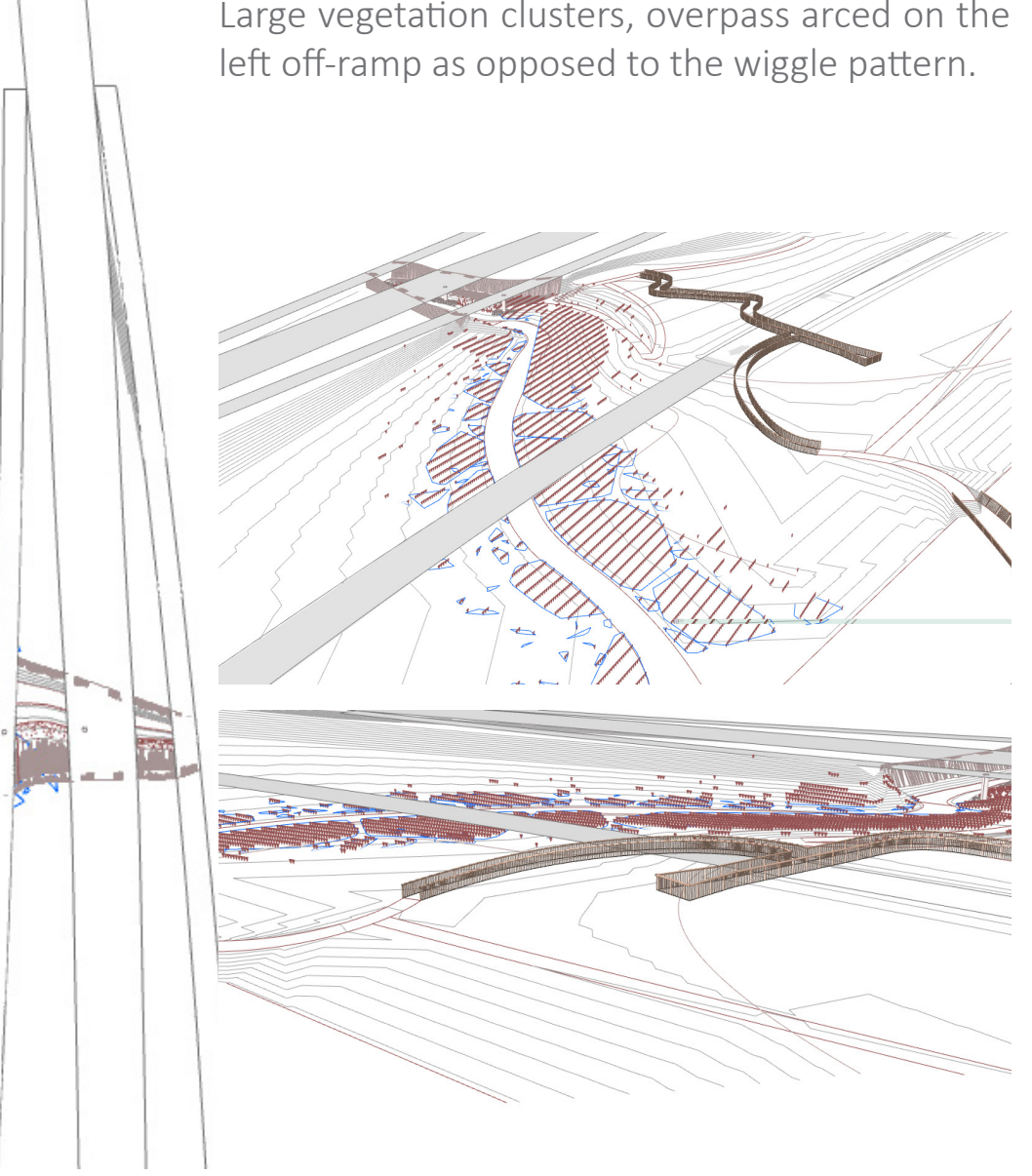

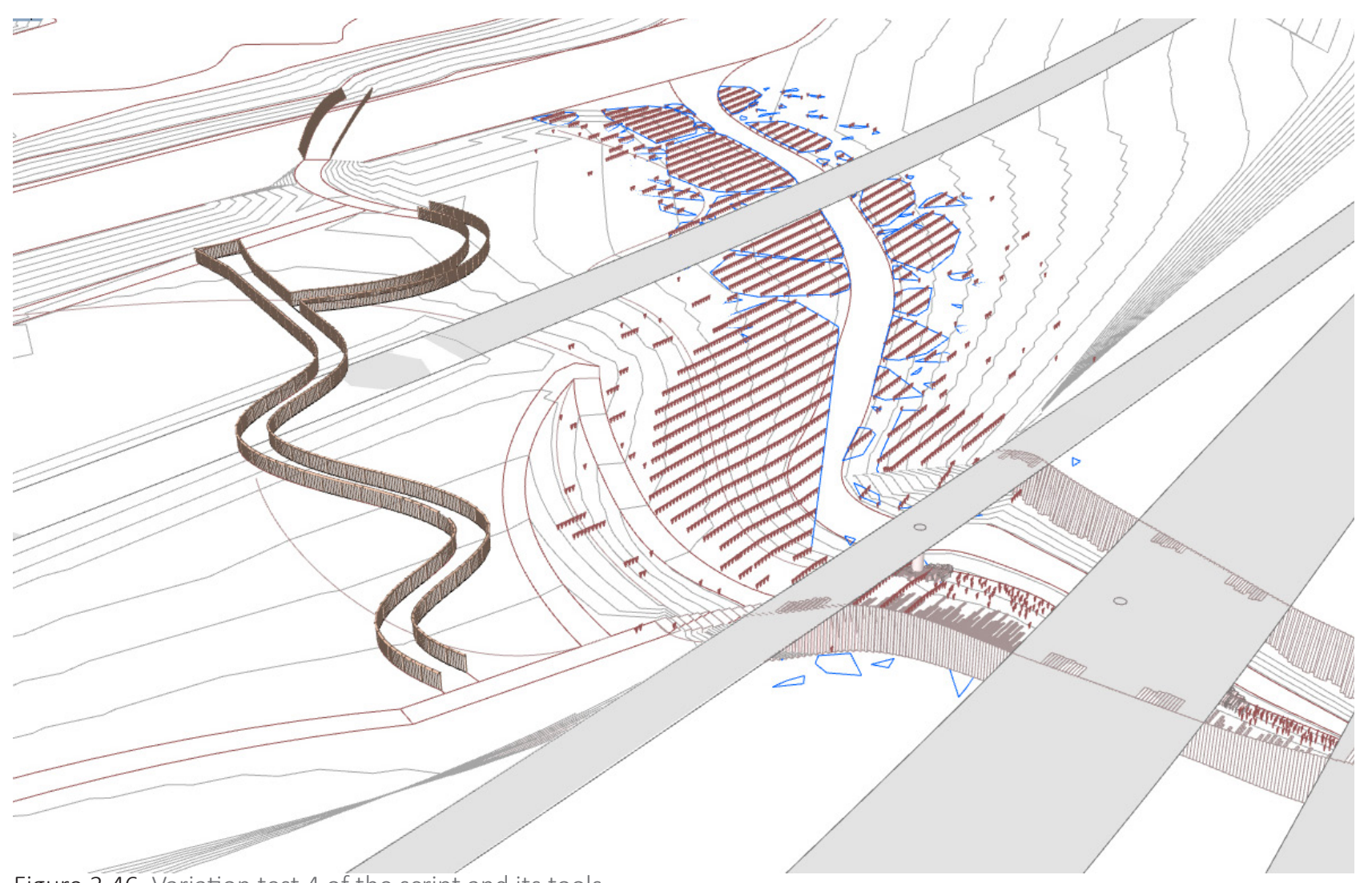

Figure.3.46. Variation test 4 of the script and its tools. 

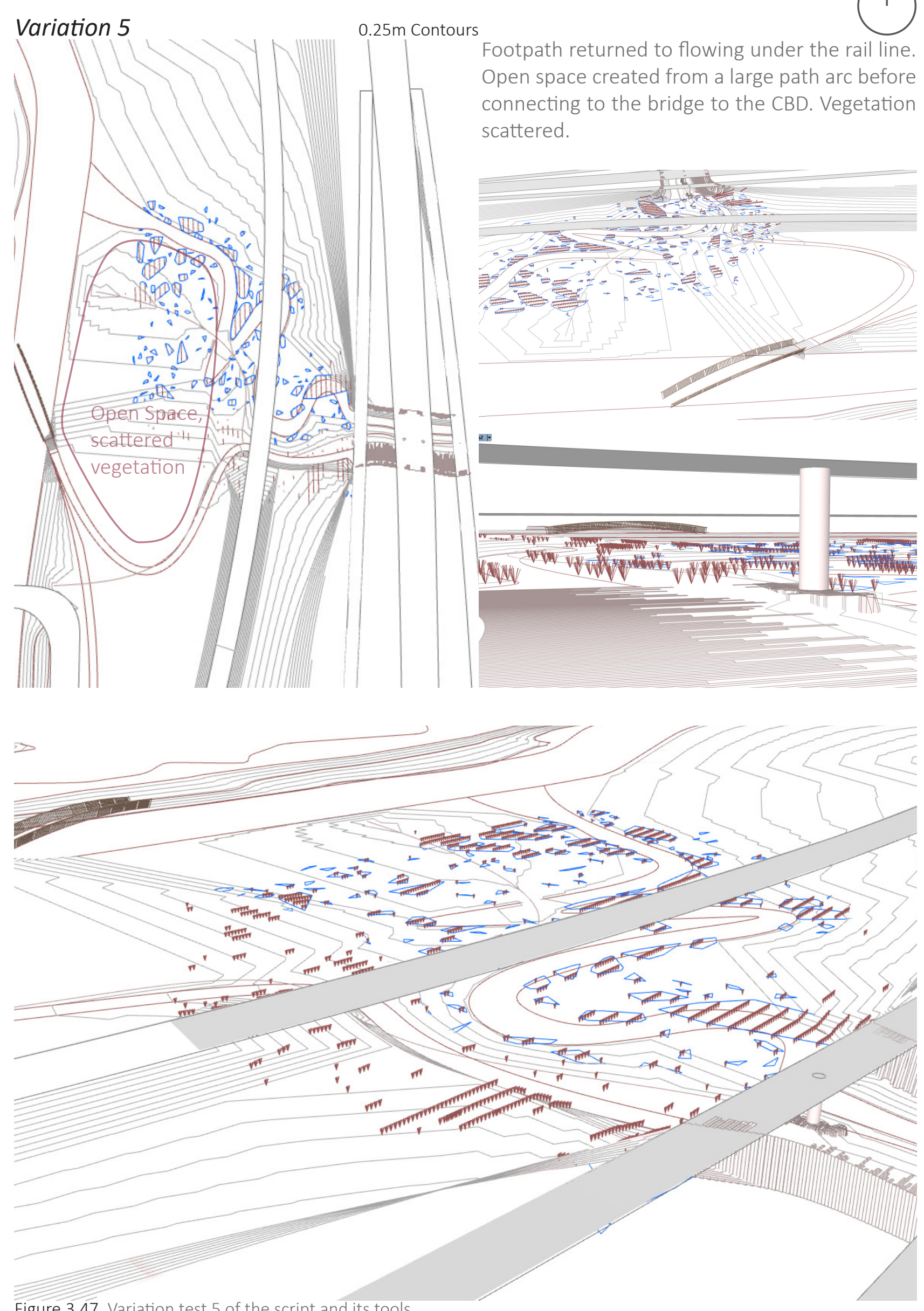

Figure.3.47. Variation test 5 of the script and its tools. 


\section{Study 3 - Conclusion}

Algorithmic thinking as a toolset enables new lens for perceiving site issues. This was apparent in the approach to the third study as there were many issues pertaining to Porirua stream, each generating ideas for site specific algorithmic toolmaking. In the case of site $\mathrm{C}$, it invoked a question of how a horizontal vector (path) can cross a range of vertical vectors (Infrastructure).

The tool that was developed reflected this question. In response, geometric relationship structuring was utilised to test variations of composition, contesting the movement of existing infrastructure with newly designed elements. This redefined the idea of the principal into a way of thinking. For instance the principals of this stage were the rail and road whereby they were under direct interrogation as these two elements closely relate to the design question. To put this in perspective, if the design brief was to investigate the path of the Porirua stream, the stream itself would likely be the principal. Principal assignment is about finding the first element in the hierarchy of the script, deciding this; frames how the script will be built. All other elements stem from the first and so the principal has the authority. Hence its' close regard for the design question.

The term generation was perceived as two different approaches within this particular use of parametrics. In the case of generating the stream, a randomised approach was utilised. With this, only the parameters in which the path could generate was defined, the parameters could be reduced or enlarged, and the intensity in the pattern of the stream could also be adjusted. Between these specifications, random forms were generated. This type of generation often leads to unexpected results however the designer sacrifices some degree of control and finesse. In the case of the rail overpass, this would generate if the rail was moved too close to the highway. This approach being a much more designed mode of generation, and better thought of as faceted generation whereby a threshold is expected or prepared to be crossed and a design response has already been programmed. The benefit of this programmed generation is that multiple design concepts can be worked on at the same time, and switched between for comparison.

While the development of this tool enabled a deep exploration into the dynamic nature of Grasshopper for the for testing of spatial variation amongst defined elements. The output of the design did not differ greatly amongst variations. This was expected as it is important to note that this tool was not aimed to generate the design, removing the role of the designer. But to stress the relationship between design potential and site and enable a mode of multi-faceted thinking in regard to the brief. As opposed to fixating down a single design path. 
This dichotomous approach allows for different variations to be compared between multiple projections of design implementation that aim for the same goal.

From a practical point of view, the potential of dynamic models such as this, while offering a fluid process to form making. Has potential for client communication, in which elements can be updated in real-time, elements can be shifted at the clients request.

What was discouraging about this tool was the dependability on the management of the script structure, for instance if one string of logics were coded poorly and as the geometries and components are linked, then editing logics means that subsequent logics must be reconfigured also - in practice this would take up valuable time. As each logic must abide by the parameters imposed by prior logics, you have to consider all possible positions where the prior element can occur in, to prepare the new element to respond accordingly. Keeping up with this dynamism is very tedious, and frustrating if the element intended is seemingly simple, such as the position of a contour.

The mystery of algorithmic tools stem from their overwhelming flexibility. Coding language is unrefined by nature, even with the ready made components that Grasshopper offers, there is endless opportunity for use. Two issues arise from this, firstly representation became tricky as generative elements had to be low quality due to computer processing power. Because of this, the vegetation generated in the model were place-holders of a single model yet representative of a diverse range of riparian species. The second issue is an issue of interface. Everything operated on sliders, which by the end, accumulated over 70 different sliders. Operating the model in this fashion felt clunky and design finesse was noticeably lost.

To summarise the key points of this study.

- Dynamic modelling incorporates a multi-faceted approach in which multiple design paths can be explored simultaneously.

- $\quad$ The Principal closely regards the design question, principal assignment is about dealing authority to specific elements and offers a way of thinking to geometric relationship structuring.

- In generative thinking, the more control the designer sacrifices to the script, the more unexpected the results.

- $\quad$ Script management is vital in a complex script, as relationships are formed logics become dependant on one another, which can lead to failure if an element is disrupted. 


\section{Thesis Conclusion}

This thesis conceived and tested two strategies for designing algorithmically. Study 1 and 3 took to the stringing of relationships between points, lines and planes, study 2 operating in feedback loops, pulling and pushing geometries and augmenting Rhino's model space, adapting the tool to the requirements of site and the ambition of the designer.

Two key findings were derived from this research, both intending to find a level of resolution to the research question.

\section{Digital Toolmaking in Landscape Architecture}

Algorithmic thinking enables landscape architects to become toolmakers, developing scripts programmed to the specific conditions of site. This is extremely applicable to the landscape architectural design process given the vast array of design problems landscape architects contend with, and the versatility that programming offers.

Over the three studies a variety of tools were developed, supporting this claim. Two primary uses of Grasshopper were used to develop such tools. Relational strategies were used in Tory Street and Porirua to test variations of site potential enabling multi a faceted approach to landscape architecture, meaning multiple design trajectories could be explored simultaneously. The dichotomy of this allows for a comparative approach between multiple options. Contrasting and projecting the spatial qualities of one option against another.

The second use, translated vegetation pattern on site into the digital model, providing a system of metric feedback. Augmenting the model space into a series of tools that themselves becoming added sense for making conceptual design, amongst the visual and intuition of the designer.

The challenge of toolmaking is translating the realities of site into points, curves and planes. One must have purpose for making the tool and a clear understanding of how the tool should operate. Hence developing an ability to think in parameters is essential, the application of parameter thinking was highlighted in all three studies through the clarification of script hierarchy, principals and logics. Three sites with comparative differences provided basis for developing this idea, although the notion of toolmaking already being conceived by authors within the literature review, this sewed the idea into the explorations of each site. The array of different uses showcased the multi-tool application of an algorithmic toolset. 


\section{Principal Thinking}

Whatisimportantto distinguish is thattoolmaking is morethan anoperative means to an end, but it encompasses a way of thinking. An algorithmic mindset, enables new frameworks for landscape architectural issues. The evolution of the principal logic and what it meant to the third study is a prime example of this. The principals (the road and rail) had authority over other elements, moving them shifted the entire model. By moving the rail between the stream and road, parameters are moved and reapplied to lower logics. Meaning the tool could investigate the positioning of the path and stream with respect to the rails new position. Principal thinking implies the linking of ideas, geometries and the conditions of site.

\section{"How can parametric software and algorithmic thinking support the conceptual design process within landscape architecture?"}

The mystery of algorithmic tools and its potential for use in landscape architecture somewhat hints an appropriate answer to the research question. The mystery exists because it is being used in a multitude of ways. Hence algorithmic toolmaking is a compelling idea. Should algorithmic thinking then be common to the landscape architects' skillset? While understanding how these tools operate is very useful to working through design issues, understanding basic principles of programming may be enough to incorporate algorithmic technique in landscape architecture, so long as a programmer with significant landscape architectural understanding is available to the translate the concept into a functioning tool, thus implying a specialized position in practice, similar to how GIS specialists are now common place. For every landscape architect to understand basic programing, better conversion can be had regarding the logistical problems of software development in regards to the landscape architectural design process and better digital tools will become available. For a landscape architect to understand programming well, the development of suitable digital tools is in their own hands.

\section{Closing Statement}

Parametric modelling can offer flexible, dynamic and exploratory perceptions of landscape architectural problems. Stressing the linking of design ideas to the conditions of site and the specifications of the brief. Points, curves and planes amalgamate form under this paradigm yet most attention is paid to the unseen relationships, parameters that guide forms conception. This new paradigm shifts thinking towards rules and sequences that can tap into the patterns of site. Advancements in this avenue of parametric use may lead to better tools for capturing 
the propinquity of life that landscape architects yearn to accommodate in their designs. The dynamic and simulative aspects of computation brings projective abilities, that enables a comparative approach to the conceptual process. Further understanding of parameter thinking and computational logics may lead to toolmaking that engages with the temporality of life. 


\section{Bibliography}

Aranda, B., \& Lasch, C. (2006). Tooling. Princeton: Princeton Architectural Press.

Cantrell, B., \& Holzman, J. (2016). Responsive Landscapes: Strategies for Responsive Technologies in Landscape Architecture. New York: Routledge.

Cantrell, B., \& Mekies, A. (2018). Codify: Parametric and Computational Design in Landscape Architecture. Routledge.

Ervin, S., \& Hasbrouck, H. (2001). Landscape Modeling: Digital Techniques for Landscape Visualisation. United States of America: The McGraw-Hill Companies Inc.

Kwinter, S., \& Boccioni, U. (1992). Landscapes of Change: Boccioni's "stati d'animo" as a General Theory of Models. The MIT Press, 50-65.

M'closkey, k., \& VanDerSys, K. (2017). Dynamic Patterns: Visualizing Landscapes in a Digital Age. New York: Routledge.

Meadows, D. H. (2008). Thinking in Systems: A Primer. Hartford, White River Junction: Chelsea Green Publishing Company.

Menges, A., \& Ahlquist, S. (2011). Computational Design Thinking. Sussex: John Wiley \& Sons Ltd.

NZ Transport Agency. (2007, December). The Design of the Pedestrian Network. Retrieved from www.Landtransport.govt.nz: https://www.nzta. govt.nz/assets/resources/pedestrian-planning-guide/docs/chapter-14. pdf

Raxworthy, J. (2017). The Discourse of the Digital in Contemporary Landscape Architecture. JoLA, 88-93.

The New Zealand Roadmakers Federation. (2012, April). Retrieved from nzrf.co.nz: http://nzrf.co.nz/techdocs/Accessible-Parking-Guide.pdf

Treib, M. (2008). Drawing/Thinking: Confronting an Electronic Age. New York: Taylor and Francis.

Vashakmadze, S. (2018). Bison For Grasshopper.

Wallis, J., \& Rahman, H. (2016). Landscape Architecture and Digital Technologies. New York: Routledge. 
Walonick, D. S. (1993). General Systems Theory. Retrieved from StatPac: https://www.statpac.org/index.htm

Westort, C. (2016). Coding Landscapes: Teaching Computer Coding to Landscape Architects. lowa State University Digital Repository, pp. 337345.

Wu, X., Zhu, X., Wu, G.-Q., \& Ding, W. (2014). Data mining with Big Data. IEEE Transactions on Knowlede and Data Engineering, 97-107. 


\section{Figure References}

All figures not on this list are the authors own images. 2019

Figure 0.01. Gardens by the Bay (Wallis \& Rahman, 2016, pp. 106-117)

Figure 0.02. Phase Shifts Park (Wallis \& Rahman, 2016, wh. 48-61)

Figure 0.03Landhausplatz (Wallis \& Rahman, 2016, wh. 16-19)

Figure 0.031. MAX Lav IV (Wallis \& Rahman, 2016, wh. 34-41)

Figure 0.04. Bellwether (Wallis \& Rahman, 2016, wh. 72-76)

Figure 0.05. South Park, Sanfrancisco (Cantrell \& Mekies, 2018, p. 8489)

Figure 0.06. Aerial view of South Park, San Francisco, California (Cantrell \& Mekies, 2018, p. 84)

Figure 0.07. (above) Site feature prioritization and weighting diagram (Cantrell \& Mekies, 2018, p. 87).

Figure.2.14. Conrad Waddington's (1957) conceptualization of an epigenetic landscape (Kwinter \& Boccioni, 1992, p. 62) 


\section{Addendum}




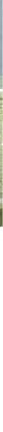




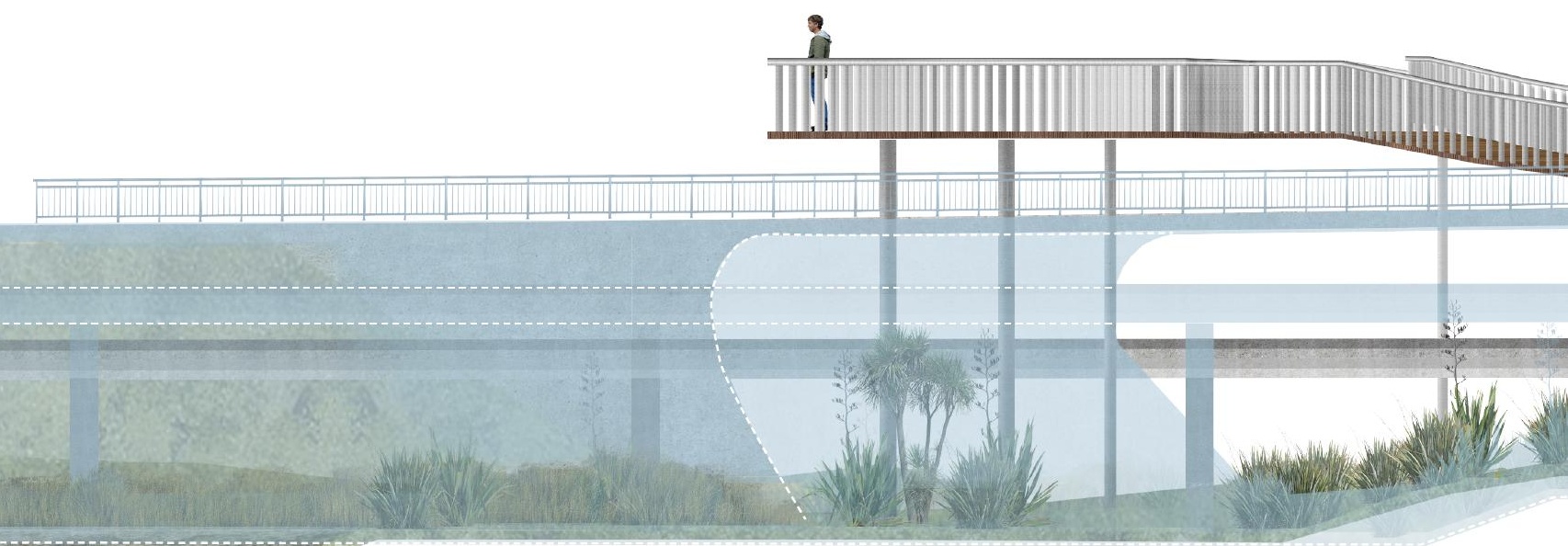

Additional Image 2. Rendered section illustrating the use of study 3's script. Further variations using the script.

Page 148|Addendum 


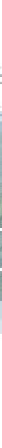

Addendum |Page 149 


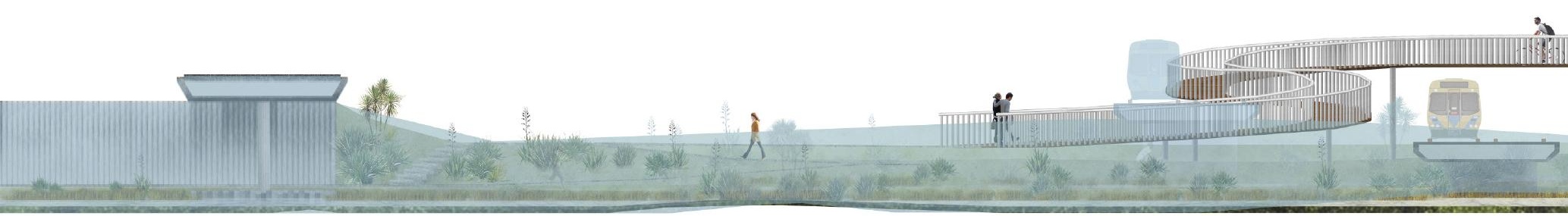

Additional Image 3. Rendered section illustrating the use of study 3's script. Further variations using the script.

Page 150|Addendum 

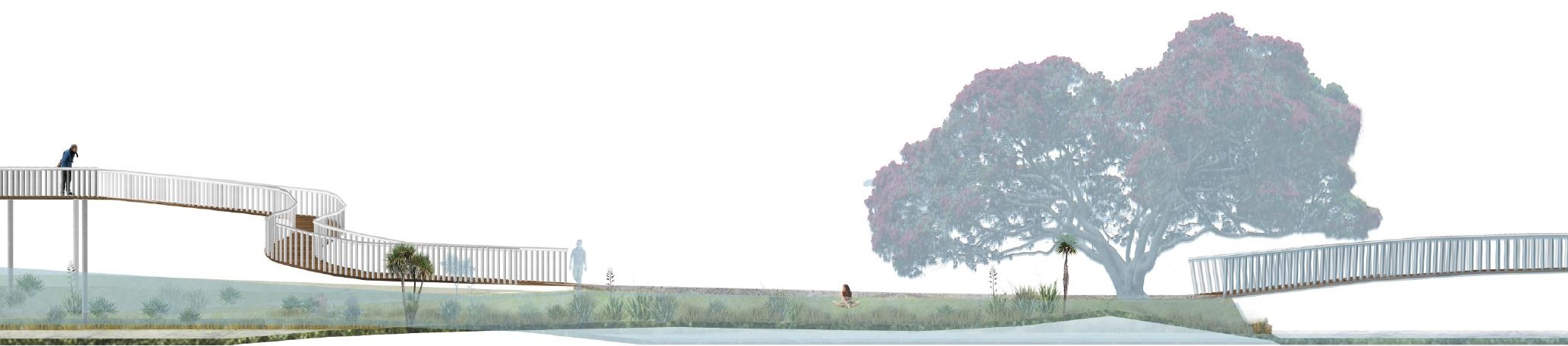

Addendum |Page 151 